João Teles de Carvalho Neto

\title{
Tomografia de Estado Quântico via Ressonância Magnética Nuclear através de Rotações Globais do Sistema de Spins
}

Tese apresentada ao Instituto de Física de São Carlos, da Universidade de São Paulo, para a obtenção do título de Doutor em Ciências "Física Aplicada".

Orientador:

Prof. Dr. Eduardo Ribeiro de Azevêdo

Co-orientador:

Prof. Dr. Tito José Bonagamba

Universidade de São Paulo

Instituto de Física de São Carlos

Departamento de Física e Informática 

Este trabalho é dedicado

À minha amada esposa Bianca, Aos meus amados pais João e Sandra, À memória do meu querido tio Carlos. 



\section{Agradecimentos}

Gostaria inicialmente de agradecer ao Prof. Dr. Eduardo R. de Azevêdo pela sua grande amizade e orientação. O estímulo e atenção despendidas foram fundamentais no meu aprendizado e na produção dos resultados constantes neste trabalho. Também agradeço pelos ensinamentos passados através de aulas e seminários. Obrigado por sua dedicação e competência exemplares.

Ao Prof. Dr. Tito J. Bonagamba também pela sua amizade e orientação. Através de seus conselhos, aulas e seminários o caminho foi percorrido mais facilmente, todavia mantendo o espírito de aventura. O seu interesse genuíno pela área de RMN serve de estímulo a todos. Obrigado pela sua confiança.

Ao Prof. Dr. Alberto Tannús pela sua amizade e orientação no meu mestrado e no meu "primeiro doutorado". Sua atuação foi fundamental na minha iniciação e desenvolvimento em ressonância. Obrigado pelo seu apoio e compreensão.

Ao Prof. Dr. Ivan S. Oliveira e ao Prof. Dr. Roberto S. Sarthour pela amizade, auxílio, colaboração e pelas aulas e seminários de Computação Quântica por RMN. Ao Prof. Dr. Philip J. Grandinetti por suas sugestões que foram bastante importantes para este trabalho. Ao Prof. Dr. José F. Schneider pelas excelentes aulas de Espectroscopia de Alta Resolução em Sólidos, as quais ajudaram a fortalecer os meus conhecimentos da área de RMN.

Ao Dr. Alviclér Magalhães pela amizade, auxílio, discussões e aulas. Agradeço tanto pelas horas de ajuda sobre tópicos variados como RMN, preparação de amostras, utilização de ${ }^{\mathrm{AT}} \mathrm{T}_{\mathrm{E}} \mathrm{Xetc}$., quanto à ajudas materiais como o fornecimento de água deuterada, tubos de ressonância, entre outros. Ao Dr. Fábio A. Bonk pela amizade, discussões e auxílio sobre os vários tópicos de CQ por RMN. Ao Dr. Gerson Mantovani pela amizade, conselhos e auxílio na preparação de amostras. Ao Dr. Edson L. G. Vidoto pela amizade, pelo desenvolvimento de sondas para CQ via RMN e pelas conversas e ensinamentos sobre instrumentação e RMN em geral. À Dr. Débora T. Balogh pela preparação e fornecimento das amostras de dodecil sulfato de sódio e dodecil sulfato de césio. Agradeço também pelo uso da infra-estrutura do laboratório de polímeros do IFSC-USP. Ao Prof. Dr. Patrick Judeinstein pelo fornecimento da amostra de perfluoroctanato de césio. Às secretárias Isabel Possatto e Nilzeli A. Nery, e aos técnicos José Carlos Gazziro, Aparecido Donizeti F. de Amorim, Odir A. Canevarollo e João G. da Silva pelos auxílios prestados ao longo do desenvolvimento do doutorado. A todos os demais professores e funcionários que direta ou indiretamente contribuíram para o desenvolvimento do meu doutorado, deixo aqui meus agradecimentos. 
Ao amigo Ruben Auccaise pela amizade, parceria, discussões e auxílio. Obrigado pela paciência, responsabilidade e esforço com que me ajudou a conduzir este trabalho. Aos amigos Carlos Brasil e Arthur Ferreira pela amizade, pelo esforço e pelo auxílio nos trabalhos realizados. Aos demais amigos com participação no grupo de ressonância: Dr. Antônio Bloes, Dr. Etelvino Novotny, Dr. José Fernando de Lima, Dr. Rogério Xavier, Dr. Roberto Franco, Dr. Bernd Foester, Ronny Ribeiro, Leandro Lopes, Fernando Paiva, Roberto Tozoni, André Bathista, Daniel Papoti, André Souza, Gregório Faria, Rodrigo Silva, Renata Lima, Guilherme Zampronio e Marcos Felipe Sampaio. A todos agradeço pela amizade e todo auxílio despendido.

Ao amigo André Gavini pela amizade, auxílio, paciência e discussões sobre os vários tópicos de CQ e CQ por RMN. Aos demais amigos do CBPF, Alexandre de Souza e Suenne Riguette pela amizade, auxílio e parceria.

Aos amigos Alexandre Ramos, Norberto Pasqua, Carlos Garrido, Ivan Marin, Valdinei Sczibor, Haroldo Fraga, Ismael Costa e Leandro Borges pela amizade duradoura.

Aos meus pais João e Sandra, por serem tão especiais e por estarem sempre presentes. Obrigado por todo amor, carinho, ajuda e dedicação. Todas as minhas realizações pessoais e profissionais são conseqüências das suas ações. Ao meu irmão Luti por toda a sua amizade, afeto e maneira de pensar que lhe fazem ser tão especial para mim. À Beth e ao Elton pela amizade e carinho e por serem parte da minha família.

À minha esposa Bianca por todo o seu amor, por todo o carinho e pela pessoa especial que ela é. Obrigado pela imensa atenção, paciência e ternura com que me ajudou a conduzir esse período de doutorado. Ao Gustavo por ser uma criança tão querida e especial, e por me ensinar várias coisas sobre tubarões brancos, baleias azuis, tiranossauro rex, etc. E à toda família Squaris Gonzalez por me acolher com tanto afeto e amizade.

Aos meus queridos avós Terezinha, Aniss e Theodolinda por serem tão queridos e especiais. Obrigado por toda a ajuda, atenção e carinho que sempre foram muito importantes para mim. Aos meus queridos padrinhos, tio Edson e tia Jane, obrigado por serem tão amigos, por toda a ajuda e por estarem sempre presentes. À família Cury Müller que sempre foi tão especial para mim, saibam que sempre estarão em meu coração. A todos os meus tios e primos, por serem tão amigos e especiais.

À população brasileira que através de agências como CAPES, CNPq, FAPESP e todas as instituições de ensino público investe arduamente no desenvolvimento do nosso país. 


\section{Resumo}

O objetivo principal da presente Tese é expor um método de Tomografia de Estado Quântico desenvolvido para ser aplicado em sistemas de núcleos quadrupolares isolados. O espaço de Hilbert de tais sistemas pode ser usado para processar a informação quântica de um sistema equivalente constituído por vários q-bits. O método proposto baseia-se na aplicação de pulsos de radiofreqüência não-seletivos que possuem a propriedade de promover rotações globais do estado quântico do sistema. Utilizando uma descrição analítica dessas rotações foi possível generalizar o método proposto para núcleos quadrupolares com qualquer número quântico de spin. O método também pode ser adaptado para sistemas de núcleos acoplados, embora para esses casos seja necessário utilizar períodos de evolução sob a hamiltoniana de interação livre para determinar alguns dos elementos da correspondente matriz densidade. Como aplicação do método de Tomografia de Estado Quântico, utilizaram-se núcleos de ${ }^{23} \mathrm{Na}$ dissolvidos em um cristal líquido liotrópico para obter os resultados experimentais das implementações do algoritmo de Deutsch e do algoritmo de busca de Grover, além da medida da dinâmica de relaxação de vários estados pseudo-puros. Também foram realizadas simulações do método proposto para o caso de um sistema quadrupolar de spin $7 / 2$ e para três spins $1 / 2$ homonucleares acoplados. 



\section{Abstract}

The main purpose of the present thesis is to propose a Quantum State Tomography method developed to be applied in quadrupolar isolated nuclei systems. The Hilbert space of such systems can be used to process the quantum information of an equivalent system formed by many qubits. The proposed method is based on the application of non-selective radiofrequency pulses that produce global rotations of the system quantum state. Using an analytical description of those rotations, it was possible to generalize the proposed method to quadrupolar nuclei with any spin quantum number. The method can also be adapted to coupled nuclear systems, although in such cases it is necessary the use of evolution periods under the free interaction hamiltonian in order to determine some of the density matrix elements. As an application of the method, ${ }^{23} \mathrm{Na}$ nuclei dissolved in a lyotropic liquid crystal were used to obtain the experimental results of the Deutsch and Grover algorithms, together with the measurement of the relaxation dynamics of some pseudo-pure states. Simulations of the proposed method applied to the quadrupolar spin $7 / 2$ nucleus and to three homonuclear coupled spin $1 / 2$ were also obtained. 



\title{
Sumário
}

\section{Lista de Figuras}

\author{
Lista de Tabelas
}

\begin{tabular}{ll}
\hline Lista de Símbolos e Abreviaturas & p. 1
\end{tabular}

\begin{tabular}{lll}
\hline & Introdução & p. 3
\end{tabular}

2 Elementos de RMN para Computação Quântica $\quad$ p.9

2.1 Etapas de Implementação $\ldots \ldots \ldots \ldots \ldots \ldots$. . . . . . . . . . 11

$2.1 .1 \quad$ Estados Pseudo-Puros $\ldots \ldots \ldots \ldots$. . . . . . . . . . 11

2.1 .2 Hamiltonianas de Interação $\ldots \ldots \ldots$. . . . . . . . . . 18

Interação de Deslocamento Químico . . . . . . . . . . . . . . . . p. 19

Interação Dipolar Magnética . . . . . . . . . . . . . . . . p. 21

Interação Dipolar Indireta ou Acoplamento Escalar . . . . . . . . . . p. p. 23

Interação Quadrupolar . . . . . . . . . . . . . . . . . . p. 24

2.1.3 Pulsos de Radiofreqüência e Gradientes de Campo Magnético . . . . p.28

2.1 .4 Leitura de Estados Quânticos . . . . . . . . . . . . . . . p. 34

2.2 SMP's . . . . . . . . . . . . . . . . . . p. 40

2.3 Relaxação de Estados Quânticos . . . . . . . . . . . . . . . . . . . p. 48

3 TEQ via Rotações Globais do Sistema de Spins $\quad$ p.57

3.1 Utilização de Pulsos não-seletivos $\ldots \ldots \ldots \ldots \ldots$. . . . . . . . . . . . . . .

3.2 Descrição Analítica das Rotações $\ldots \ldots \ldots$. . . . . . . . . . . . . 61 
3.3 Seleção de Coerências via Média Temporal $\ldots \ldots \ldots$. . . . . . . . p. 68

3.4 Aplicações . . . . . . . . . . . . . . . . . . . p. 70

3.4.1 Sistemas de Spins Quadrupolares Isolados . . . . . . . . . . . p. 70

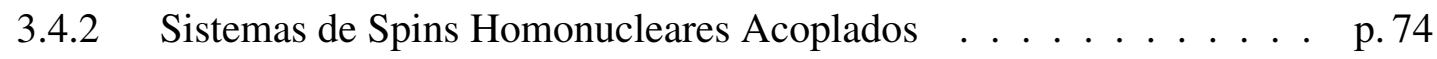

Sistema de equações com tempo de evolução livre . . . . . . . . p. 75

Simulação da TEQ em um sistema homonuclear $\ldots$. . . . . . . p.76

3.4 .3 Sistemas de Spins Heteronucleares Acoplados . . . . . . . . . . p. 79

4 Implementação Experimental do Método de TEQ $\quad$ p. 81

$4.1 \quad$ A Amostra de Cristal Líquido $\ldots \ldots \ldots \ldots \ldots$. . . . . . . . . . . 81

4.2 Sequiências de Pulsos e Procedimentos de Calibração . . . . . . . . . . . . . p. 87

$4.3 \quad$ Validação experimental do método de TEQ $\ldots \ldots \ldots \ldots$. . . . . . . . p. 97

4.4 Aplicações do método de TEQ . . . . . . . . . . . . . . . . . . . p. 110

$4.4 .1 \quad$ Criação dos Estados de Bell . . . . . . . . . . . . . . . . p. 110

$4.4 .2 \quad$ Algoritmo de Deutsch . . . . . . . . . . . . . p. 113

4.4 .3 Algoritmo de Grover . . . . . . . . . . . . . . . p. 116

4.4 .4 Experimentos de Relaxação . . . . . . . . . . . . . . . . p. 122

$\begin{array}{lll}5 \text { Conclusões } & \text { p. } 129\end{array}$

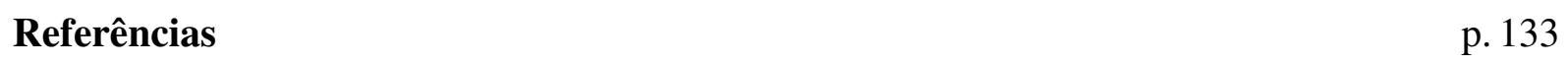

\begin{tabular}{|ll}
\hline A Apêndices & p. 137
\end{tabular}

A.1 Descrição didática do método de TEQ . . . . . . . . . . . . . . . . . . . p. 137

A.2 Códigos de otimização dos SMP's . . . . . . . . . . . . . . . . . p. 141

A.3 Exemplo de macro utilizada para criação de porta lógica . . . . . . . . . . . p. 145

A.4 Código da seqüência de pulsos utilizada para realizar os experimentos de CQ p. 148

A.5 Código para reconstrução da matriz densidade a partir dos experimentos de TEQ . . . . . . . . . . . . . . . . . . . . 156 
A.6 Macros para programar e ler a sequiência de experimentos usados no processo

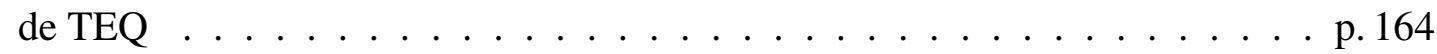

A.7 Resultados experimentais da TEQ $\ldots \ldots \ldots \ldots \ldots \ldots \ldots$. . . . . . . 166

A.8 Tensores irredutíveis e funções de Wigner para spin 3/2 . . . . . . . . . . . . p. 169 



\section{Lista de Figuras}

$1 \quad$ Níveis de energia magnéticos de um dipolo nuclear, $\mu=\gamma \mathbf{I}$, na presença de um campo magnético $\mathbf{B}_{\mathbf{0}}$ e a respectiva indexação dos estados lógicos . . . . p. 12

2 Comportamento de $\epsilon$ e $\varepsilon$ em função da temperatura para um núcleo de spin $1 / 2$ em equilíbrio térmico $\ldots \ldots \ldots \ldots \ldots$ p. . . . . . . . . . . . . . . . . .

3 Pureza do estado de equilíbrio em função da polarização para $1 \leq N \leq 6$

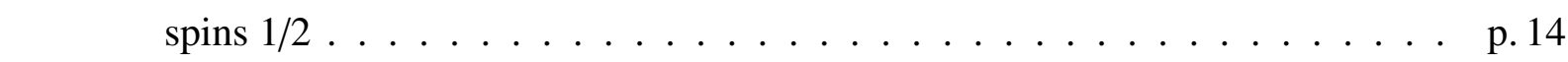

4 Exemplo de indexação lógica em um sistema de spin $3 / 2 \ldots \ldots$. . . . . . p. 15

5 Acoplamento dipolar magnético direto entre dois núcleos $\ldots \ldots \ldots$. . . . p. 21

$6 \quad$ Níveis de energia e transições permitidas para dois spins $1 / 2$ heteronucleares

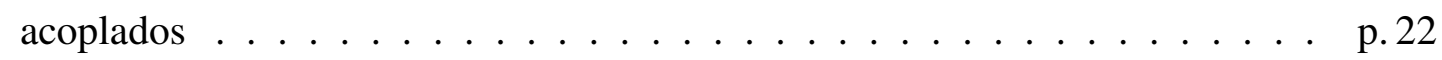

7 Seqüência de pulsos que produz a hamiltoniana de exchange (2.64) para a ordem mais baixa da expansão de Magnus . . . . . . . . . . . . . . p. 32

8 Medida das componentes reais e imaginárias das linhas espectrais na condição de alta resolução . . . . . . . . . . . . . . . . . p. 39

$9 \quad$ Ilustração da forma de um pulso SMP $\ldots \ldots \ldots \ldots$. . . . . . . . . . . . . .

10 Representação pictórica do processo de otimização no espaço de parâmetros . p.45

11 Ilustração do funcionamento do algoritmo de otimização Simplex $\ldots$. . . . . p.46

12 Exemplo da indexação $\{r i\}$ e $k$ (negrito) para as várias espécies nucleares que formam o sistema de spins . . . . . . . . . . . . . . p.59

13 Gráfico das funções de Wigner reduzidas, $d_{m^{\prime}, 2}^{2}(-\theta)$, para $m^{\prime}=1$ e $m^{\prime}=-1$. . p.67

14 Simulação do método de tomografia para um sistema de spin $7 / 2 \ldots$. . . . . p.73

15 Dependência da matriz densidade com a ordem de coerência $m$ dos operadores $\mathrm{T}_{l m}^{L}$ para os casos de $N=2$ e $N=3$ spins $1 / 2$ acoplados $\ldots \ldots$. . . . p. 74 
16 Gráfico da evolução da projeção dos estados de rank 0 nos estados de rank 2 devido à ação da hamiltoniana livre . . . . . . . . . . . . . . . . . . . . p p.78

17 Simulação do método de tomografia para um sistema de 3 spins $1 / 2$ homonucleares . . . . . . . . . . . . . . . . . . . . . p. 79

18 Exemplos de mesofases de um cristal líquido termotrópico $\ldots \ldots$. . . . . p. 82

19 Dimensões e geometria do posicionamento da amostra de cristal líquido . . . p. 84

$20 \quad$ Snapshot do painel do software VNMR $\ldots \ldots \ldots \ldots$. . . . . . . . 92

21 Diagrama da seqüência de pulsos e parâmetros relacionados à calibração do "computador quântico" . . . . . . . . . . . . . . . . p. 93

22 Diagrama da seqüência de pulsos e parâmetros relacionados à programação do"computador quântico" . . . . . . . . . . . . . . . . . . . . p. 94

23 Fluxograma do processo de programação e tomografia realizados no espectrômetro de RMN. . . . . . . . . . . . . . . . . . . . . . p. 96

24 Estado de superposição em $\tau=0$ tomografado experimentalmente e criado para a observação da dinâmica dos elementos de $\rho$ sob a hamiltoniana quadrupolar $\mathrm{H}_{Q} \ldots \ldots \ldots \ldots \ldots \ldots \ldots \ldots$ p. . . . . . . . . . . . . . . . .

25 Diagrama da seqüência de pulsos contendo a etapa de evolução livre utilizada para estudar a dinâmica da matriz densidade . . . . . . . . . . . . . . . p. 99

26 Efeito de erro de fase na tomografia da coerência $\rho_{13}$ evidenciado pela oscilação do módulo . . . . . . . . . . . . . . . . . . . p. 100

27 Resultado experimental da dinâmica dos elementos da matriz densidade que oscilam com a freqüência quadrupolar $\omega_{Q} \ldots \ldots$. . . . . . . . p. 101

28 Resultado experimental da dinâmica dos elementos da matriz densidade que não oscilam com a freqüência quadrupolar $\omega_{Q} \ldots$. . . . . . . . . . . . . p. 102

29 Estados proporcionais à base de operadores irredutíveis de polarização $\mathrm{T}_{l m}+$

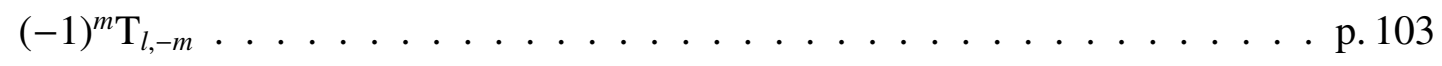

\begin{tabular}{|ll||l|l|}
\hline 30 Continuação dos resultados da Figura 29 & contendo os operadores de ordem 0. & p. 104 \\
\hline
\end{tabular}

31 Variação das amplitudes espectrais com o ângulo de nutação para os estados das Figuras $29|\mathrm{e}| 30 \ldots \ldots$. . . . . . . . . . . . . . . . . 105 
32 Sequiência de pulsos simples utilizada para a criação de estados com coerências de ordens 2 e $3 \ldots \ldots \ldots \ldots$. . . . . . . . . . . . . . . . . . . . . . . .

33 Evolução dos ranks da matriz densidade sob a hamiltoniana quadrupolar . . . p. p. 107

34 Resultado da tomografia dos experimentos de criação de coerências de $2^{a}$ e $3^{a}$ ordem . . . . . . . . . . . . . . . . . . . p. 109

35 Circuito quântico usado para criar a base de estados de Bell. . . . . . . . . . p. 111

36 Tomografia das etapas do circuito da Figura $35 \mid$ para a criação dos estados de

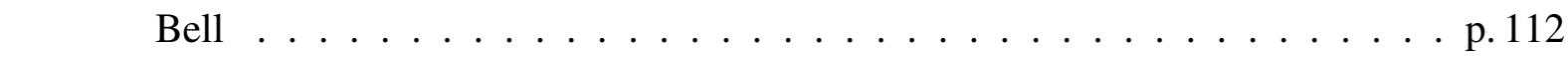

37 Tomografia do estado do Gato, $|00\rangle+|11\rangle \ldots \ldots$. . . . . . . . . p. 113

38 Circuito quântico que implementa o algoritmo de Deutsch. . . . . . . . . . . p. 114

39 Tomografia das etapas do circuito da Figura 38|| para a implementação do algoritmo de Deutsch . . . . . . . . . . . . . . . p. 115

40 Circuito quântico que implementa o algoritmo de Grover . . . . . . . . . . . p. 117

41 Tomografia do estado inicial de superposição no algoritmo de Grover, $|\psi\rangle=$ $|00\rangle+|01\rangle+|10\rangle+|11\rangle \ldots \ldots \ldots \ldots \ldots \ldots$. . . . . . . . . . . . . . . . .

42 Tomografia do algoritmo de Grover para as operações $\mathrm{G}_{00} \mathrm{e}_{01}$. . . . . . p. 120

43 Continuação da tomografia do algoritmo de Grover para as operações $\mathrm{G}_{10} \mathrm{e}$

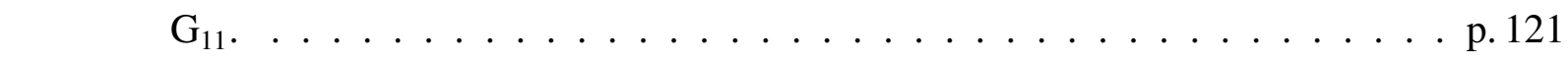

44 Tomografia da dinâmica de relaxação do estado de superposição da Figura 41|| $\mid$ p. 123

45 Tomografia da dinâmica de relaxação do estado de superposição da Figura 41|| sem pulso de refocalização . . . . . . . . . . . . . . . . . . . p. 124

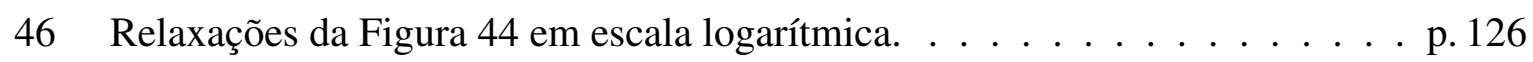

47 Tomografia da dinâmica de relaxação das populações dos estados pseudodiagonais correspondentes à base computacional . . . . . . . . . . . . . . . . p. 127

48 Tomografia da dinâmica de relaxação das populações do estado pseudo-diagonal correspondente ao estado do Gato. . . . . . . . . . . . . . . . . . . . . p. 128

49 Ilustração do processo de caracterização de um estado quântico puro. . . . . . . p. 138

50 Ilustração do processo de caracterização de um estado quântico misto . . . . p. p. 139

51 Ilustração do método de tomografia proposto por Bonk et al. [1]. . . . . . . . . . p. 140 
52 Ilustração do método de tomografia proposto nesta tese e que utiliza somente pulsos não-seletivos de curta duração. . . . . . . . . . . . . . . . . . . p. 141

53 Resultados experimentais da TEQ relacionados ao experimento de criação de estados de Bell. . . . . . . . . . . . . . . . . . . . . p. 166

54 Resultados experimentais da TEQ relacionados à implementação do algoritmo de Deutsch. . . . . . . . . . . . . . . . . . . . . . p. 167

55 Resultados experimentais da TEQ relacionados à implementação do algoritmo de Grover. . . . . . . . . . . . . . . . . . . . . . . . p. 168

$56 \quad$ Tensores irredutíveis $\mathrm{T}_{l m}$ de $\operatorname{rank} l$ e ordem $m$ para o spin $3 / 2 . \ldots$. . . . . . p. 169

$57 \quad$ Funções $d_{1, m}^{l}$ de Wigner para o spin $3 / 2 . \ldots \ldots \ldots$. . . . . . . p. 170 


\section{Lista de Tabelas}

1 Indexação dos tensores irredutíveis que expandem o espaço de operadores para o caso de um sistema formado por três spins $1 / 2$ acoplados $\ldots . . \quad$ p. 64

$2 \quad$ Fases dos pulsos de rf, $\phi$, e do receptor, $\alpha$, necessárias para tomografar os

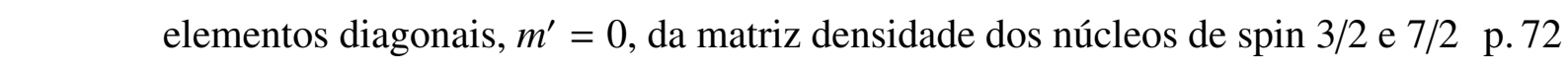

3 Acoplamentos escalares $J_{i j}$ e deslocamentos químicos $\omega_{0}^{i}-\bar{\omega}_{0}$ (diagonal) para o sistema homonuclear simulado de 3 spins $1 / 2 \ldots \ldots$. . . . . . . . . 77

4 Fases $\phi$ dos pulsos de rf e fases $\alpha$ do receptor para os experimentos de seleção

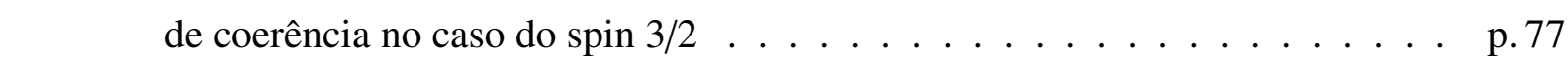

5 Ângulos de nutação $\theta$, em radianos, que maximizam a sensibilidade espectral

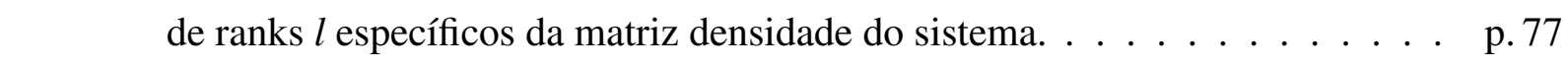

6 Estimativas dos desvios experimentais dos parâmetros para cada segmento

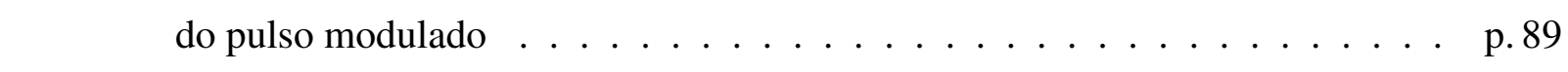

7 Propriedades numéricas das portas lógicas criadas via técnica SMP e utilizadas no experimento de criação dos estados de Bell . . . . . . . . . . . . . p. 111

$8 \quad$ Fidelidades experimentais obtidas da TEQ de cada passo do experimento de criação de estados de Bell. . . . . . . . . . . . . . . . p. 111

$9 \quad$ Propriedades numéricas das portas lógicas criadas via técnica SMP e utiliza-

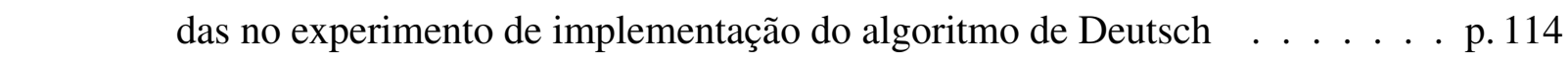

10 Fidelidades experimentais obtidas da TEQ de cada passo do experimento de implementação do algoritmo de Deutsch. . . . . . . . . . . . . p. 116

11 Propriedades numéricas dos operadores de Grover criados via técnica SMP e

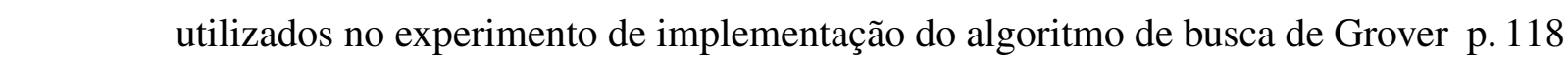

12 Fidelidades experimentais obtidas em cada uma das 5 iterações dos operadores de Grover. . . . . . . . . . . . . . . . . . . . . p. 119 



\section{Lista de Símbolos e Abreviaturas}

CQ (Computação Quântica), p. 3

RMN (Ressonância Magnética Nuclear), p. 3

DSS (Dodecil Sulfato de Sódio), p. 3

TEQ (Tomografia de Estado Quântico), p. 3

$\rho$ (Operador Densidade), p. 4

SMP (Strongly Modulating Pulses), , p. 6

rf (radiofrequiência), p. 18

DQ (Deslocamento Químico), p. 19

SEP (Sistema de Eixos Principais), p. 20

TI (Tensor Irredutível), p. 25

THM (Teoria da hamiltoniana Média), p. 31

CL (cristal líquido), p. 81 


\section{1 \\ Introdução}

O presente trabalho surgiu com o objetivo de expandir o tamanho dos sistemas estudados em Computação Quântica $(C Q)$ por Ressonância Magnética Nuclear $(R M N)$ no Grupo de RMN do Instituto de Física de São Carlos (IFSC). Através da colaboração entre os Professores Eduardo R. de Azevêdo e Tito J. Bonagamba do IFSC, os Professores Roberto S. Sarthour, Ivan S. Oliveira e Alberto P. Guimarães do Centro Brasileiro de Pesquisas Físicas (CBPF) e o Professor Jair C. C. Freitas da Universidade Federal do Espírito Santo (UFES), foi possível estabelecer uma parceria envolvendo um bom número de estudantes de graduação e pós-graduação e resultando em trabalhos publicados [1-5], duas teses defendidas até o momento [6,7] e um livro publicado [8], além de artigos e trabalhos de divulgação. Entre os sistemas experimentais utilizados pelo grupo estão os cristais líquidos liotrópicos. Tais sistemas fornecem o ambiente químico anisotrópico necessário para que núcleos com spin maior que $1 / 2$ em solução possam ser usados em CQ por RMN. Além disso, a orientação das estruturas do cristal líquido na presença do forte campo magnético estático de RMN, possibilita a observação de linhas laterais estreitas devido ao acoplamento do momento de quadrupolo elétrico nuclear com o gradiente de campo elétrico produzido pela distribuição eletrônica local. Utilizando um cristal líquido liotrópico formado pela molécula de Dodecil Sulfato de Sódio $(D S S)$ foi possível realizar vários estudos de CQ com 2 bits quânticos (q-bits) implementados pelos 4 estados magnéticos do núcleo de sódio (spin 3/2) [1-3]. Entre os trabalhos realizados com essa amostra está a chamada Tomografia de Estado Quântico (TEQ) .

A técnica de TEQ é uma ferramenta importante nos estudos de CQ, pois possibilita descrever o operador densidade completo associado ao estado do sistema. Técnicas experimentais que realizam medidas projetivas sobre estados quânticos fornecem em geral a informação sobre as amplitudes dos auto-estados na base do operador de medida. Em técnicas que realizam medidas sobre um ensemble de estados, como é o caso da RMN, é possível obter simultaneamente a informação sobre conjuntos específicos de coerências da correspondente matriz densidade. No entanto, em ambos os casos, não se obtém de forma direta os demais graus de liberdade que ca- 
racterizam um sistema quântico, cujo estado mais geral corresponde a uma mistura estatística, a qual é descrita pelo operador densidade $(\rho)$. Para obter a informação completa sobre $\rho$ é necessário aplicar uma série de transformações, via as hamiltonianas de controle características de cada sistema, para que os valores de todas as coerências possam ser acessados.

O ponto de partida do presente trabalho foi o método de tomografia proposto por Bonk et al. [1] Nesse trabalho, os autores observaram que aplicando-se uma ciclagem de fase do tipo CYCLOPS [9], a qual é rotineiramente usada para corrigir imperfeições na detecção do sinal de RMN, obtém-se um espectro que depende somente dos elementos diagonais da matriz densidade. Como o núcleo de sódio no cristal líquido de DSS possui 3 linhas espectrais e as populações da correspondente matriz densidade de desvio possuem 3 elementos linearmente independentes, foi possível determinar o valor das populações resolvendo-se o respectivo sistema linear exato. Para obter o valor das coerências de $\rho$, foram utilizados pulsos seletivos de radiofreqüência (rf) para promover a transição dessas coerências para a posição das populações. Obviamente, uma vez que as populações assumem somente valores reais, a parte imaginária e real de cada coerência necessitam ser transferidas separadamente. Dessa forma, utilizando-se pulsos seletivos e a ciclagem CYCLOPS foi possível obter todos os elementos da matriz densidade de desvio. O passo seguinte era tentar adaptar o método de TEQ do spin 3/2 para o spin 7/2. Portanto, os primeiros desenvolvimentos deste trabalho foram no sentido de encontrar uma ciclagem específica que produzisse um espectro dependente somente dos valores diagonais, em analogia ao caso do spin 3/2. Assim, após um processo heurístico de investigação foi possível encontrar a ciclagem desejada para o caso de spin 7/2. A Tabela 2 no capítulo 3 contém essa ciclagem de fases juntamente com a ciclagem CYCLOPS. O segundo passo na adaptação do método de TEQ foi tentar desenvolver os pulsos seletivos necessários para promover a transferência das coerências para a diagonal. Após algumas observações sobre a maneira como pulsos não-seletivos atuam sobre o estado do sistema, foi feita a hipótese da possibilidade de poder gerar as transferências desejadas utilizando-se somente os pulsos não-seletivos. Pulsos não-seletivos, se forem suficientemente curtos, possuem a propriedade de produzir rotações globais sobre o sistema de spins. Por serem bem mais curtos que os pulsos seletivos eles apresentam vantagens com relação ao tempo de duração da operação de leitura, a qual pode ser limitada pelo tempo de relaxação dos elementos de $\rho$. Além disso, operações de rotação são mais robustas do ponto de vista experimental, uma vez que suas propriedades dependem somente do pulso de rf e não dos parâmetros internos da hamiltoniana do sistema. Outra hipótese importante levantada no desenvolvimento desta tese, foi sobre a necessidade de fazer-se a transferência das coerências para a diagonal. Uma vez que o sinal de RMN corresponde ao valor das coerências de $1^{a}$ ordem, foi feita a tentativa de transferir os valores das demais coerências dire- 
tamente para a posição dessa coerência. Assim, novamente após uma investigação heurística foi possível encontrar algumas combinações de fase e amplitude de pulsos não-seletivos que selecionavam algumas coerências na posição das coerências de $1^{a}$ ordem. Os resultados dessa etapa do trabalho foram apresentados no $10^{\circ}$ encontro da Associação de Usuários de Ressonância Magnética Nuclear (AUREMN) [10]. Naquela ocasião tive a oportunidade de conversar com o Professor Philip. J. Grandinetti, o qual sugeriu a utilização da base de tensores esféricos irredutíveis na representação da matriz densidade do sistema. Após ler alguns artigos indicados pelo Prof. Grandinetti $[11,12]$ e aproveitando a experiência dos Professores Bonagamba e Azevêdo $[13,14]$ no uso de tensores irredutíveis para descrever as hamiltonianas de interação em sistemas sólidos, foi possível estabelecer um método de TEQ que utiliza somente pulsos não-seletivos e que em princípio pode ser aplicado para qualquer spin não-acoplado. A partir desse ponto optou-se por estabelecer a teoria geral para a TEQ em sistemas quadrupolares isolados com qualquer número quântico de spin. Uma tentativa de descrição mais didática do método proposto encontra-se no Apêndice A.1.

Pode-se dividir o método proposto em dois conceitos principais. O primeiro diz respeito às operações de rotação do sistema de spins, e o segundo corresponde ao conceito de seleção de coerências através da ciclagem de fases dos pulsos de radiofreqüência. As fases dos pulsos correspondem aos eixos em torno dos quais as rotações do sistema são realizadas. Através do uso de combinações adequadas dessas fases é possível realizar a leitura de conjuntos específicos de coerências separadamente. O objetivo principal desta tese é expor de uma maneira analítica e precisa o funcionamento desse método original e mostrar aplicações experimentais do mesmo a estudos de CQ por RMN. Os desenvolvimentos realizados neste trabalho também podem ser apreciados na ref. [5].

Um segundo desenvolvimento realizado neste trabalho correspondeu ao desenho das sequências de pulsos necessárias para realizar as operações quânticas de interesse em computação quântica. A abordagem até então usada pelo grupo baseava-se na aplicação de sequiências de pulsos de rf, cujos efeitos sobre o sistema de spins eram conhecidos, para tentar construir as portas lógicas utilizadas nos algoritmos de CQ. Desse desenvolvimento, foi possível construir operações lógicas básicas que foram implementadas na amostra de DSS [1,3]. No entanto, para operações lógicas gerais, torna-se difícil adaptar as seqüências conhecidas de RMN para os problemas de CQ. Esse problema é particularmente proeminente nos sistemas quadrupolares, em que são usados spins maiores do que 1/2 para implementar um sistema de múltiplos q-bits. Nesses sistemas, a hamiltoniana de interação não possui uma forma simples em termos de produtos de operadores dos espaços de spin 1/2 correspondentes. Para tentar resolver esse problema, adotou-se um procedimento de otimização numérica dos pulsos de rf baseado no trabalho de 
Fortunato et al. [15]. Nesse trabalho, os autores propuseram a técnica de pulsos fortemente modulados, Strongly Modulating Pulses (SMP), para otimizar operações de rotação em spins específicos de sistemas homonucleares. Posteriormente, Kampermann e Veeman [16] utilizaram os SMP's para produzir portas lógicas em estudos de CQ no núcleo de sódio em uma amostra sólido-cristalina de $\mathrm{NaNO}_{3}$. Nesta tese, os SMP's foram empregados não só na otimização de portas lógicas, mas também na preparação de estados pseudo-puros para a implementação de algoritmos quânticos na amostra de DSS. Como resultado, foi possível implementar o algoritmo de Deutsch e o algoritmo de busca de Grover para 2 q-bits. O primeiro podendo ser apreciado também na ref. [5]. Aplicando o método de TEQ proposto neste trabalho, foi possível seguir todos os passos dos algoritmos através da evolução das correspondentes matrizes densidade.

O método de TEQ também pôde ser aplicado ao estudo da relaxação do estado quântico dos núcleos de sódio na amostra de DSS. Foi possível verificar o comportamento previsto pela matriz de relaxação de Redfield, em que, devido à natureza da hamiltoniana do sistema, todas as coerências apresentam um decaimento mono-exponencial enquanto que as populações relaxam de forma multi-exponencial. A relação entre os diferentes tempos de decaimento com os mecanismos de relaxação característicos dessa amostra não são tratados neste trabalho, sendo que o trabalho de doutorado do estudante Ruben Auccaise, sob orientação do Prof. Sarthour e co-orientação do Prof. Azevêdo, deverá conter informações mais detalhadas sobre esses processos.

Esta tese encontra-se organizada em 5 capítulos. O primeiro e o último correspondem tradicionalmente à Introdução e à Conclusão do trabalho. O capítulo 2 contém tanto os conceitos básicos quanto as ferramentas teórico-experimentais utilizadas para a realização dos experimentos. O capítulo 3 trata especificamente do método de tomografia. Esse é essencialmente um capítulo teórico que culmina em aplicações do método a vários tipos de sistemas. Para ilustrar essas aplicações são apresentadas algumas simulações numéricas. Por fim, o capítulo 4 contém as aplicações experimentais. Esse capítulo inicia-se com uma descrição da amostra de cristal líquido utilizada e segue descrevendo as características instrumentais e de programação do espectrômetro onde foram realizadas as medidas de RMN. Em seguida são expostos os resultados das tomografias realizadas ao longo da implementação dos experimentos de criação de estados de Bell, algoritmo de Deutsch e algoritmo de busca. Para finalizar, são apresentadas as tomografias das relaxações de alguns estados pseudo-puros.

Entre as convenções usadas neste trabalho está o uso de variáveis em negrito sem itálico para designar vetores e variáveis sem itálico e normalmente maiúsculas para designar operadores quânticos. A exceção dessa convenção é para o operador densidade que é designado pelas 
letras gregas $\rho$ ou $\sigma$. O operador correspondente à hamiltoniana do sistema é sempre chamado diretamente de "hamiltoniana". As demais variáveis escalares são escritas em geral em itálico e sem negrito. Por fim, funções matemáticas são escritas sem itálico e sem negrito. 


\section{2 \\ Elementos de RMN para Computação Quântica}

Neste capítulo são discutidos os tópicos fundamentais relacionados à implementação de experimentos de computação quântica em RMN. Embora essa seja uma aplicação mais específica da RMN e até mesmo da computação quântica, ainda assim trata-se de um assunto vasto e com ampla produção bibliográfica. Assim sendo, este capítulo procura focar na descrição teórica e experimental apenas dos conceitos mais fundamentais e dos métodos mais utilizados na literatura, dando um pouco mais de ênfase nos conteúdos utilizados neste trabalho. Artigos de revisão sobre o assunto podem ser encontrados em $[17,18]$. Um tratamento mais completo sobre CQ e CQ por RMN pode ser obtido através dos livros [8, 19, 20].

A técnica de Ressonância Magnética Nuclear tem proporcionado métodos bastante úteis na demonstração de operações lógicas quânticas e na caracterização do estado quântico dos sistemas de spins. O sucesso da aplicação da RMN em estudos de CQ se deve à grande capacidade de controle da dinâmica dos spins nucleares através do uso de pulsos de radiofreqüência. A tecnologia atual na área de eletrônica de radiofrequiência permite a modelagem de pulsos com resolução temporal bastante superior aos tempos de evolução associados às principais interações presentes nos estudos de RMN. Aliado ao controle sobre a dinâmica estão os tempos de relaxação relativamente longos dos estados magnéticos nucleares, os quais possibilitam a realização de um bom número de operações antes que transformações não-unitárias atuem sobre o estado quântico do sistema. Devido a essas características, a RMN obteve sucesso na preparação de estados iniciais, na implementação de portas lógicas quânticas e na leitura dos estados quânticos. Muitos dos métodos teóricos e experimentais empregados nos estudos de CQ por RMN se beneficiaram da longa tradição que a técnica possui na manipulação dos spins, incluindo aí a possibilidade de excitação e detecção de múltiplas coerências em amostras líquidas e sólidas. Características como essas estão na essência do método de TEQ proposto nesta tese. 
Uma possível listagem dos pré-requisitos necessários para que um sistema experimental obtenha sucesso na implementação de um processador quântico, pode ser encontrada no trabalho de DiVincenzo [21], a qual é reproduzida abaixo:

1. Um sistema físico que possa ser escalonado para um grande número de q-bits bem caracterizados.

2. A capacidade de iniciar os q-bits em um estado bem determinado, e.g., $|00 \ldots\rangle$.

3. Tempos de descoerência longos o suficiente para que um número grande de operações quânticas possa ser realizado durante esses tempos.

4. Um conjunto de portas lógicas quânticas universais.

5. A capacidade de medir o estado de q-bits individuais.

Todas as técnicas experimentais propostas até agora falham em implementar a maioria dos requisitos acima. No entanto, a técnica de RMN é a que chegou mais longe em termos de processamento de informação quântica, como pode ser visto por exemplo na implementação do algoritmo de fatoração de Shor [22] em um sistema de 7 q-bits, realizada por Vandersypen et al. [23]. Todavia, a mesma sofre restrições sérias quanto a sua escalabilidade, não cumprindo portanto o item 1 da lista acima. Uma dessas restrições diz respeito à perda de sensibilidade com o aumento do tamanho do sistema de spins, assunto que é comentado ao final da sub-seção 2.1.1. Para uma crítica mais completa sobre a utilidade da RMN em CQ vide ref. [24]. No entanto, o fato de que futuros processadores quânticos não venham a ser baseados puramente na técnica de RMN tradicional, não impede que estudos acadêmicos sejam realizados. De fato, muitos modelos de teoria de informação quântica já puderam ser testados experimentalmente pela técnica. Além disso, é possível que outras técnicas experimentais se beneficiem, ao menos em parte, dos desenvolvimentos realizados em CQ por RMN [25-27].

A seção 2.1 contém o material mais fundamental deste capítulo, em que são tratadas as etapas de implementação dos experimentos de CQ em RMN. A seção 2.2 embora também possa ser considerada uma etapa de implementação, merece uma atenção especial, pois todas as implementações experimentais de portas lógicas e estados pseudo-puros descritas nesta tese foram feitas com a técnica exposta nessa seção. A seção 2.3 trata da relaxação dos estados dos spins nucleares. 


\subsection{Etapas de Implementação}

As etapas de implementação foram divididas em três partes básicas. A primeira corresponde ao procedimento de preparação dos estados pseudo-puros, em que são descritas as técnicas que permitiram o uso da RMN tradicional na implementação de experimentos de computação quântica. A segunda etapa trata da dinâmica dos estados quânticos proporcionada pelas interações nucleares mais comuns em RMN. Em especial, a interação quadrupolar é discutida com um pouco mais de detalhe juntamente com as características da amostra de cristal líquido utilizada. Por fim, a etapa de leitura dos estados quânticos trata do processo de medida em RMN e discute como a magnetização da amostra pode trazer informação sobre esses estados.

\subsubsection{Estados Pseudo-Puros}

Para realizar CQ por RMN, são normalmente utilizadas moléculas que contenham átomos com momento angular nuclear igual a 1/2. Para um único núcleo de spin $1 / 2$ submetido a um campo magnético $B_{0}$ intenso, ocorre a separação da energia magnética nuclear, $E=-\mu \cdot \mathbf{B}_{\mathbf{0}}$, em dois níveis de energia: $E_{0}=-\frac{1}{2} \hbar \gamma B_{0}$ e $E_{1}=\frac{1}{2} \hbar \gamma B_{0}$, sendo $\gamma$ a constante giromagnética nuclear. Essa é a chamada interação Zeeman. Aos respectivos auto-estados associa-se a base computacional $|0\rangle$ e $|1\rangle$, vide Figura 11(a). Assim, uma molécula que contenha $N$ spins $1 / 2$ interagentes pode formar um sistema de $N$ q-bits descrito no espaço de Hilbert $\varepsilon_{1} \otimes \ldots \otimes \varepsilon_{N}$ formado pelo produto do espaço $\varepsilon_{i}$ de cada núcleo. Uma quantidade macroscópica de moléculas à temperatura ambiente é usada em um experimento de RMN, de tal forma que todas as manipulações e medidas dos estados nucleares correspondem a uma média sobre todas as moléculas, vide Figura 1(b). Tal procedimento é conhecido como computação quântica de ensemble [28, 29]. Pode-se enxergar o processo como se cada molécula, formando um sistema de $n$ q-bits, fosse uma cópia do computador quântico.

Assumindo certas condições normalmente encontradas em RMN, pode-se mostrar [30] que as populações dos spins do sistema em equilibro térmico com a amostra seguem a distribuição de Boltzmann. Dessa forma, a matriz densidade de equilíbrio para um único spin $1 / 2$ corresponde a:

$$
\rho=\frac{1}{e^{\varepsilon}+e^{-\varepsilon}}\left[\begin{array}{cc}
e^{\varepsilon} & 0 \\
0 & e^{-\varepsilon}
\end{array}\right]
$$

Em que a razão entre as energias magnética e térmica, $\varepsilon=\frac{\hbar \gamma B_{0}}{2 k T}$, será chamada de fator de Boltzmann, sendo $T$ a temperatura da amostra e $k$ a constante de Boltzmann. 
a)

$$
\begin{aligned}
& E=-\mu \cdot \mathbf{B}_{\mathbf{0}} \\
& \frac{|1\rangle}{|0\rangle}+\frac{1}{2} \hbar \gamma B_{0} \\
& -\frac{1}{2} \hbar \gamma B_{0}
\end{aligned}
$$

b)

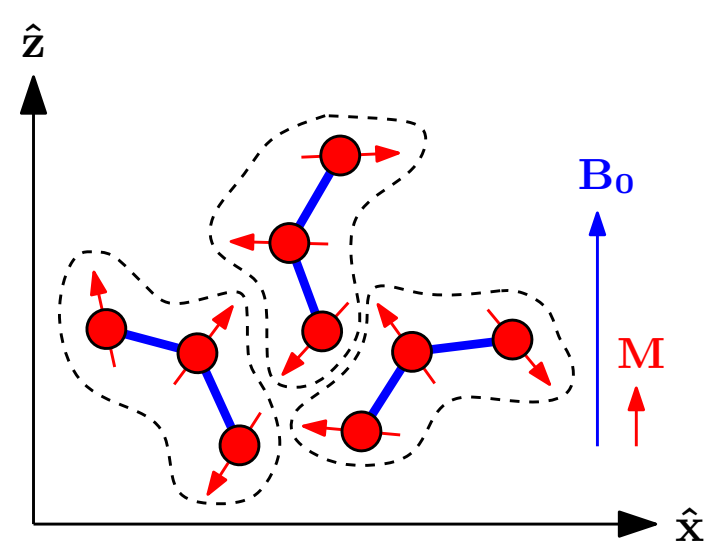

Figura 1: (a) Níveis de energia magnéticos de um dipolo nuclear, $\mu=\gamma \mathbf{I}$, na presença de um campo magnético $\mathbf{B}_{\mathbf{0}}$ e a respectiva indexação dos estados lógicos. (b) Ilustração do ensemble de moléculas, cujos spins nucleares formam o sistema quântico usado para processar informação quântica. A magnetização M contém a informação sobre o estado médio de todas as moléculas.

Definindo a grandeza polarização $\epsilon$ como sendo a diferença de populações de $\rho$, encontra-se que a polarização de equilíbrio é dada por:

$$
\epsilon=\frac{e^{\varepsilon}-e^{-\varepsilon}}{e^{\varepsilon}+e^{-\varepsilon}}=\tanh (\varepsilon)
$$

Expressando a matriz densidade de equilíbrio em função da polarização, encontra-se:

$$
\rho(\epsilon)=\frac{1}{2}\left[\begin{array}{cc}
1+\epsilon & 0 \\
0 & 1-\epsilon
\end{array}\right]=\frac{1}{2} \mathbb{1}_{2}+\epsilon \mathrm{I}_{z} .
$$

Para um sistema de $N$ spins, a matriz densidade de equilíbrio corresponde ao estado produto:

$$
\rho_{e q}=\bigotimes_{i=1}^{N}\left(\frac{1}{2} \mathbb{1}_{2}+\epsilon \mathrm{I}_{z}\right)=\frac{1}{2^{N}} \mathbb{1}_{2^{N}}+\Delta \rho_{e q}(\epsilon)
$$

Em que $\mathrm{I}_{z}$ é a componente $z$ adimensional do operador momento angular e $\Delta \rho_{e q}(\epsilon)$, conhecida como matriz densidade de desvio, é o operador que contém todos os termos diferentes da identidade e portanto possui traço nulo. Para os campos magnéticos e temperaturas típicas 11 dos experimentos atuais de RMN encontramos $\varepsilon \approx 10^{-5}$, o que torna a aproximação $\epsilon=\varepsilon$ bastante boa. A Figura 2 mostra como essa aproximação se mantém mesmo para temperaturas da ordem de $10^{-1} \mathrm{~K}$. Nessas situações de baixa polarização, $\epsilon \approx 10^{-5}$, pode-se desprezar os termos de $\mathrm{O}\left(\epsilon^{2}\right)$, obtendo-se:

$$
\Delta \rho_{e q}(\epsilon)=\frac{1}{2^{N-1}} \sum_{i=1}^{N} \epsilon \mathrm{I}_{z}^{i} \quad \text { para } \quad \epsilon \ll 1 .
$$

\footnotetext{
${ }^{1}$ Campo magnético $B_{0} \approx 10 \mathrm{~T}$ e temperatura $T \approx 300 \mathrm{~K}$.
} 
Fator de Boltzmann $\varepsilon$ e polarização $\epsilon$ para 1 q-bit

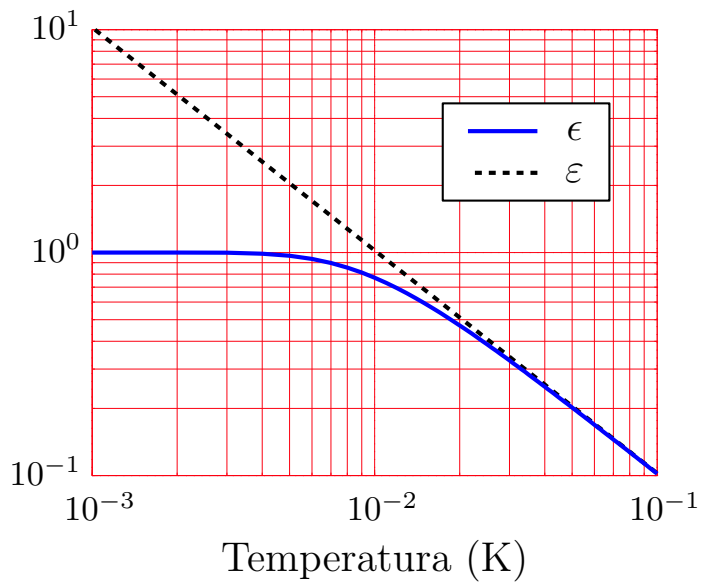

Figura 2: Comportamento de $\epsilon$ e $\varepsilon$ em função da temperatura para um núcleo de spin $1 / 2$ em equilíbrio térmico. Para o cálculo do gráfico foi utilizado o $\gamma$ do núcleo de ${ }^{1} \mathrm{H}$ em um campo de $10 \mathrm{~T}$.

$\mathrm{I}_{z}^{i}$ é o operador $\mathrm{I}_{z}$ do $i$-ésimo núcleo no espaço dos $N$ spins. Definindo a pureza de um estado como sendo o traço do quadrado do operador densidade, $p=\operatorname{Tr}\left\{\rho^{2}\right\}$, pode-se mostrar que a pureza do estado (2.4) é dada por:

$$
p=\operatorname{Tr}\left\{\rho_{e q}^{2}\right\}=\left(\frac{1+\epsilon^{2}}{2}\right)^{N} .
$$

Assim, obtém-se para um estado puro $p=1$ e para uma mistura estatística máxima $p=2^{-N}$. A Figura 3 mostra os valores de $p$ em função de $\epsilon$ para vários valores de $N$. Vê-se portanto que os estados de equilíbrio térmico em RMN tradicional estão longe de serem estados puros, não cumprindo com o requisito número 2 de DiVincenzo. Contudo, se for possível transformar o estado $\rho_{e q}$ em um estado $\bar{\rho}$ da forma:

$$
\bar{\rho}=\frac{1}{2^{N}}(1-q) \mathbb{1}_{2^{N}}+q|\psi\rangle\langle\psi|
$$

então é possível realizar qualquer experimento de CQ considerando somente o termo $|\psi\rangle\langle\psi|$. Isso pois, o termo identidade é invariante sob transformações unitárias, podendo portanto ser desprezado. O estado $|\psi\rangle\langle\psi|$ assim criado é chamado de estado pseudo-puro e o fator $q$ é um número real compreendido entre 0 e 1 . O estado de desvio de (2.7) corresponde a $\Delta \bar{\rho}=$ $q\left(|\psi\rangle\langle\psi|-\frac{1}{2^{N}} \mathbb{1}_{2^{N}}\right)$ e é esse estado que normalmente é computado nos experimentos de RMN. O motivo disso é apresentado na sub-seção 2.1.4. 
Pureza em função da polarização para $N$ de 1 até 6 q-bits

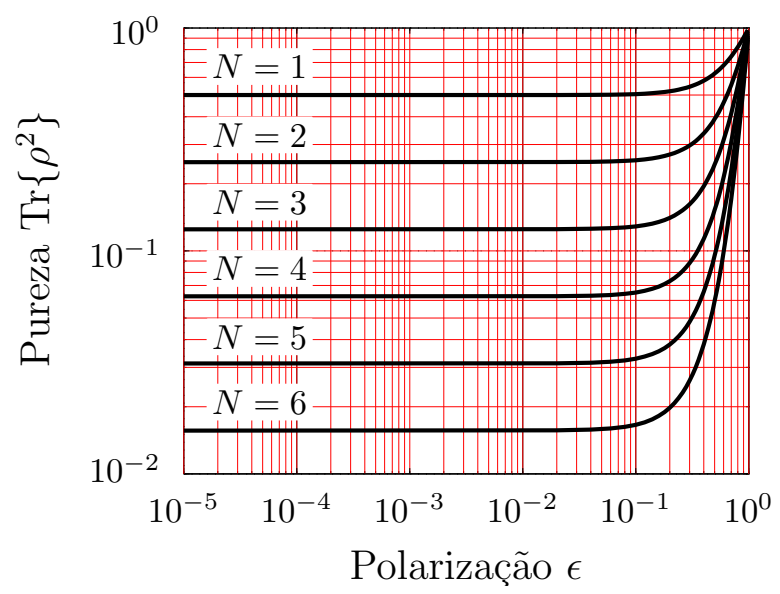

Figura 3: Pureza do estado de equilíbrio em função da polarização para $1 \leq N \leq 6$ spins $1 / 2$. Observa-se que mesmo com polarizações de aproximadamente $10^{-1}$ encontra-se praticamente o valor mínimo de pureza.

Analisando a possibilidade de transformar $\rho_{e q}$ em $\bar{\rho}$ vê-se que para um único spin $1 / 2 \mathrm{o}$ estado pseudo-puro de um único q-bit é naturalmente obtido:

$$
\rho_{\text {eq }}=\frac{1}{2} \mathbb{1}_{2}+\epsilon \mathrm{I}_{z}=\frac{1}{2}(1-\epsilon) \mathbb{1}_{2}+\epsilon|0\rangle\langle 0|
$$

No entanto, para $N$ spins acoplados o estado $\rho_{e q}-\frac{1}{2^{N}}(1-q) \mathbb{1}_{2^{N}}$, em geral, não possui os mesmos auto-valores de $q|\psi\rangle\langle\psi|$. Logo, transformações unitárias ${ }^{2}$ não resolvem o problema de transformar $\rho_{e q}$ em $\bar{\rho}$. Portanto, é necessário encontrar alguma transformação não-unitária que realize essa tarefa. Uma possibilidade é aplicar um procedimento de média sobre vários estados transformados, tal que:

$$
\bar{\rho}=\frac{1}{M} \sum_{\mu=1}^{M} \mathrm{U}_{\mu} \rho_{e q} \mathrm{U}_{\mu}^{\dagger}=\frac{1}{2^{N}}(1-q) \mathbb{1}_{2^{N}}+q|\psi\rangle\langle\psi| .
$$

Por exemplo, para o caso de baixa polarização $\epsilon$, o vetor de auto-valores de $\Delta \rho_{e q}$ para 2 spins $1 / 2$ é igual a $\frac{\epsilon}{2}[1,0,0,-1]$. Nesse caso, o número mínimo de permutações para gerar um pseudo-puro é igual a 3:

$$
\begin{aligned}
\Delta \bar{\rho} & =\frac{\epsilon}{6}([1,0,0,-1]+[1,0,-1,0]+[1,-1,0,0]) \\
& =\frac{\epsilon}{6}[3,-1,-1,-1]=\frac{2 \epsilon}{3}\left(|00\rangle\langle 00|-\frac{1}{4} \mathbb{1}_{4}\right) .
\end{aligned}
$$

\footnotetext{
${ }^{2}$ Transformações unitárias preservam os auto-valores de um operador hermitiano, fazendo com que a permutação dos mesmos seja a transformação unitária mais geral.
} 

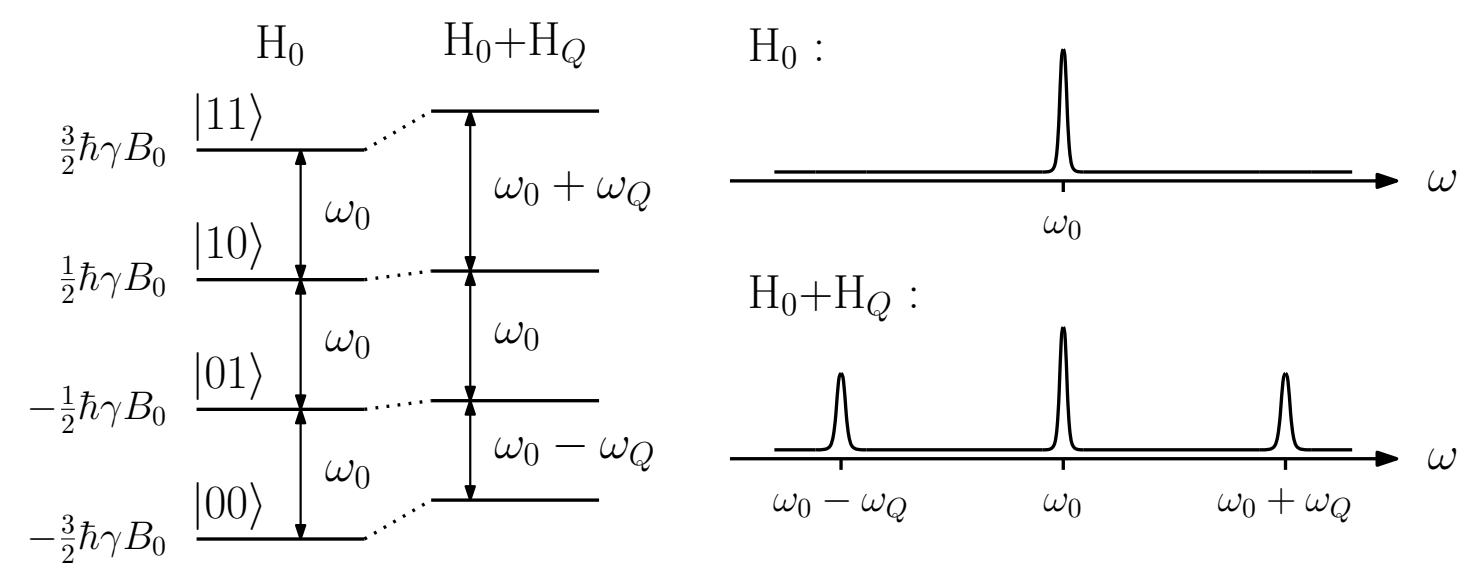

Figura 4: Exemplo de indexação lógica em um sistema de spin 3/2. A hamiltoniana $\mathrm{H}_{Q}$ representa a interação quadrupolar necessária para que se possa acessar todos os estados do sistema. As linhas espectrais à direita correspondem às transições entre niveis energéticos adjacentes, vide sub-seção 2.1.4.

Vale notar que o valor de $\epsilon$ pode não ser o mesmo para diferentes spins ${ }^{3}$. No caso de dois spins $1 / 2$ com polarizações $\epsilon_{1}$ e $\epsilon_{2}$, o vetor de auto-valores será $\frac{1}{2}\left[\epsilon_{+}, \epsilon_{-},-\epsilon_{-},-\epsilon_{+}\right]$, sendo que $\epsilon_{ \pm}=\epsilon_{1} \pm \epsilon_{2}$. Nesse caso, poderiam ser usadas as seguintes permutações:

$$
\begin{aligned}
\Delta \bar{\rho} & =\frac{1}{12}\left(\left[\epsilon_{+}, \epsilon_{-},-\epsilon_{-},-\epsilon_{+}\right]+\left[\epsilon_{+},-\epsilon_{-}, \epsilon_{-},-\epsilon_{+}\right]+\left[\epsilon_{+},-\epsilon_{+}, \epsilon_{-},-\epsilon_{-}\right]+\right. \\
& \left.+\left[\epsilon_{-},-\epsilon_{+},-\epsilon_{-}, \epsilon_{+}\right]+\left[\epsilon_{-}, \epsilon_{+},-\epsilon_{-},-\epsilon_{+}\right]+\left[\epsilon_{-},-\epsilon_{-},-\epsilon_{+}, \epsilon_{+}\right]\right) \\
& =\frac{2 \epsilon_{1}}{3}\left(|00\rangle\langle 00|-\frac{1}{4} \mathbb{1}_{4}\right) .
\end{aligned}
$$

Outra possibilidade de criar um sistema de $N$ q-bits em RMN é utilizando-se núcleos com spin maior que $1 / 2$. Os estados magnéticos de um núcleo com spin $I$ em um campo $B_{0} \hat{\mathbf{z}}$ correspondem aos auto-valores do operador $\mathrm{I}_{z}: I, I-1, \ldots,-I-1,-I$. Assim, pode-se associar os estados lógicos de um sistema de $N$ q-bits aos estados magnéticos de um núcleo com spin $I=\frac{1}{2}\left(2^{N}-1\right)$. Na verdade, não basta somente a existência de um campo magnético para a utilização desse sistema em CQ. Como será visto nas sub-seções 2.1.2 e 2.1.4, é necessário que as freqüências de transição entre níveis adjacentes de energia sejam distintas para que o sistema acesse todos os estados possíveis do espaço de Hilbert e para que seja possível a medida de todos esses estados. A interação que normalmente se presta a essa função é o acoplamento do momento de quadrupolo elétrico nuclear, existente em núcleos com $I>1 / 2$, com o gradiente de campo elétrico na vizinhança nuclear. Por esse motivo tais núcleos são chamados de núcleos quadrupolares. A Figura 4 ilustra a utilização de um núcleo de spin 3/2 para representar um sistema de dois q-bits.

\footnotetext{
${ }^{3}$ Espécies nucleares diferentes possuem constantes giromagnéticas distintas.
} 
Utilizando os mesmos princípios empregados para obter a Equação (2.1), o operador densidade de equilíbrio de um núcleo de spin I possui o seguinte vetor de auto-valores:

$$
\left[\rho_{I}\right]_{i=j}=\frac{\left[e^{I \varepsilon^{\prime}}, e^{(I-1) \varepsilon^{\prime}}, \ldots, e^{-I \varepsilon^{\prime}}\right]}{e^{I \varepsilon^{\prime}}+e^{(I-1) \varepsilon^{\prime}}+\ldots+e^{-I \varepsilon^{\prime}}} .
$$

Em que $\varepsilon^{\prime}$ corresponde ao dobro do fator de Boltzmann: $\varepsilon^{\prime}=2 \varepsilon=\frac{\hbar \gamma B_{0}}{k T}$.

Pode-se mostrar que o estado de equilíbrio de um núcleo de spin I com fator de Boltzmann $\varepsilon^{\prime} / 2$ corresponde ao estado de equilíbrio de $N=\log _{2}(2 I+1)$ núcleos de spin $1 / 2$ com polarizações $\tanh \left(\varepsilon^{\prime}\right), \tanh \left(2 \varepsilon^{\prime}\right), \ldots, \tanh \left(2^{N-1} \varepsilon^{\prime}\right)$ cada. Ou seja:

$$
\rho_{I}=\bigotimes_{i=1}^{N}\left[\frac{1}{2} \mathbb{1}_{2}+\tanh \left(2^{N-i} \varepsilon^{\prime}\right) \mathrm{I}_{z}\right]
$$

Talvez exista algum interesse prático na expressão (2.13) em estudos que procuram relacionar a dinâmica de sistemas quadrupolares com sistemas equivalentes de spin $1 / 2$. No entanto esse assunto não é tratado neste trabalho, sendo que o resultado (2.13) é apresentado aqui apenas como uma maneira de tentar relacionar a polarização de núcleos de spin $1 / 2$ com o estado de equilíbrio de um núcleo de maior dimensão.

Voltando à Equação (2.12), tem-se que na aproximação de baixo $\varepsilon^{\prime}$, normalmente satisfeita em RMN, obtém-se uma expressão análoga ao caso de 1 spin 1/2:

$$
\rho_{I}=\frac{1}{2 I+1}\left(\mathbb{1}_{2 I+1}+\varepsilon^{\prime} \mathrm{I}_{z}\right) \quad \text { para } \quad \varepsilon^{\prime} \ll 1
$$

Em que agora $\mathrm{I}_{z}$ é a componente $\mathrm{z}$ do operador momento angular para o spin $I$.

Assim, um sistema de 2 q-bits pode em princípio ser realizado por um núcleo de spin 3/2, 3 q-bits por um spin 7/2 e assim sucessivamente. Da mesma forma que para o caso de spins $1 / 2$ acoplados, os auto-valores do operador densidade de equilíbrio de um núcleo quadrupolar não correspondem aos auto-valores do operador (2.7). Portanto, alguma transformação não-unitária também é necessária. Por exemplo, para um spin 3/2, pode-se usar as seguintes permutações para produzir um estado pseudo-puro:

$$
\begin{aligned}
\Delta \bar{\rho}_{3 / 2} & =\frac{\epsilon^{\prime}}{24}([3,1,-1,-3]+[3,-3,1,-1]+[3,-1,-3,1]) \\
& =\frac{\epsilon^{\prime}}{8}[3,-1,-1,-1] \\
& =\frac{\epsilon^{\prime}}{2}\left(|00\rangle\langle 00|-\frac{1}{4} \mathbb{1}_{4}\right) .
\end{aligned}
$$


Embora essas médias de operações de permutação sejam suficientes para produzir pseudopuros, elas não correspondem às transformações não-unitárias mais gerais possíveis. Na prática, existem transformações experimentalmente mais convenientes que produzem os mesmos estados pseudo-puros obtidos pelos procedimentos 2.10), 2.11) e 2.15). Por exemplo, no trabalho de Khitrin et al. [31] é proposto um esquema de criação do estado $|00\rangle\langle 00|$ em um núcleo de spin 3/2 em que é utilizada a operação unitária:

$$
\mathrm{U}=\frac{1}{\sqrt{2}}\left[\begin{array}{cccc}
2 & 0 & 0 & 0 \\
0 & 1 & 0 & i \\
0 & 0 & 2 & 0 \\
0 & i & 0 & 1
\end{array}\right]
$$

seguida por uma transformação não-unitária $\mathcal{G}$ de média espacial, tal que:

$$
\mathcal{G} \mathrm{U} \cdot\left[\begin{array}{cccc}
3 & 0 & 0 & 0 \\
0 & 1 & 0 & 0 \\
0 & 0 & -1 & 0 \\
0 & 0 & 0 & -3
\end{array}\right] \cdot \mathrm{U}^{\dagger}=\mathcal{G}\left[\begin{array}{cccc}
3 & 0 & 0 & 0 \\
0 & -1 & 0 & -2 i \\
0 & 0 & -1 & 0 \\
0 & 2 i & 0 & -1
\end{array}\right]=\left[\begin{array}{cccc}
3 & 0 & 0 & 0 \\
0 & -1 & 0 & 0 \\
0 & 0 & -1 & 0 \\
0 & 0 & 0 & -1
\end{array}\right]
$$

Conforme é comentado na sub-seção 2.1.3, a transformação U é obtida utilizando-se pulsos seletivos de duplo quantum. A transformação $\mathcal{G}$ corresponde à aplicação de um gradiente de campo magnético que produz um processo de média análogo ao indicado na Equação [2.9], com a diferença de que o índice da somatória tende a uma variável contínua que varre as diferentes posições dos spins na amostra. Caso não seja possível utilizar a transformação $\mathcal{G}$, pode-se fazer a média da transformação $U$ com a transformação inversa $U^{\dagger}$, produzindo o mesmo resultado de (2.17).

Uma outra possibilidade de geração de estados pseudo-puros é através da chamada indexação lógica [28]. Nesse caso, parte do número total de q-bits do sistema é usada para fazer a computação de interesse e parte é usada para fazer a indexação dos estados que realmente correspondem a um pseudo-puro. A indexação lógica possui a característica de usar somente operações unitárias, no entanto paga-se o alto preço, ao menos nos tempos atuais, de se comprometer parte dos q-bits que poderiam ser utilizados para realizar a computação de interesse. Um exemplo desse procedimento pode ser visto considerando-se um sistema de 3 spins 1/2 da mesma espécie nuclear, ou seja, mesmo $\epsilon$. Nesse caso é possível aplicar uma operação unitária de permutação U sobre o estado de equilíbrio tal que:

$$
\Delta \bar{\rho} \propto \mathrm{U} \cdot[3,1,1,-1,1,-1,-1,-3] \cdot \mathrm{U}^{\dagger}=[3,-1,-1,-1,1,1,1,-3] .
$$


Dessa forma, operando-se somente no subespaço dos quatro primeiros ou quatro últimos elementos obtém-se um sistema de dois q-bits pseudo-puros. Na Equação 2.18) foram escritos somente os elementos diagonais de $\Delta \bar{\rho}$.

Na sub-seção 2.1.4 é mostrado que a intensidade $S$ do sinal da amostra em um experimento de RMN, na aproximação de baixa polarização, é proporcional ao fator que multiplica os operadores $\mathrm{I}_{z}$ em (2.5) e em (2.14). Portanto:

$$
S \propto \frac{\varepsilon}{2^{N-1}} \quad \text { para } \quad \varepsilon \ll 1
$$

Com isso, por mais que o requisito número 2 de DiVincenzo tenha sido satisfeito através do emprego dos estados pseudo-puros, o requisito número 1 não é alcançado. Isso ocorre pois, pela Equação 2.19], um aumento linear no número de q-bits implica em uma perda exponencial na intensidade do sinal, tornando a técnica de RMN não escalonável.

Embora nesta sub-seção tenham sido considerados operadores densidade com polarização arbitrária, nos desenvolvimentos que seguem nesta tese será subentendido somente o caso de baixa polarização em que as Equações (2.5) e (2.14) descrevem corretamente o estado de equilíbrio térmico.

\subsubsection{Hamiltonianas de Interação}

Para que a técnica de RMN seja bem sucedida na implementação de experimentos de CQ é importante que as hamiltonianas do sistema possam produzir um conjunto universal de portas lógicas, em conformidade com o requisito número 4 de DiVincenzo. O controle necessário para produzir as operações lógicas é possível em RMN através do uso dos chamados pulsos de radiofreqüência $(r f)$, denotados por $\mathbf{B}_{\mathbf{1}}$ ou $\mathbf{B}_{\mathbf{r f}}$. Esses são pulsos de campo magnético com direção perpendicular à direção do campo magnético principal $\mathbf{B}_{\mathbf{0}}$. Adotando-se a direção $z$ como sendo a direção de $\mathbf{B}_{\mathbf{0}}$, o campo de rf possui a forma geral:

$$
\mathbf{B}_{1}(t)=B_{1 x}(t) \hat{\mathbf{x}}+B_{1 y}(t) \hat{\mathbf{y}}
$$

Assim, calculando-se a hamiltoniana a partir da energia potencial magnética entre o campo magnético total e os dipolos nucleares, $E=-\sum_{i=1}^{N} \mu_{i} \cdot \mathbf{B}$, encontra-se:

$$
\mathrm{H}(t)=-\hbar \sum_{i=1}^{N}\left[\omega_{0}^{i} \mathbf{I}_{z}^{i}+\omega_{1 x}^{i}(t) \mathbf{I}_{x}^{i}+\omega_{1 y}^{i}(t) \mathbf{I}_{y}^{i}\right]
$$

Em que $\omega_{0}^{i}=\gamma_{i} B_{0}, \omega_{1 x}^{i}=\gamma_{i} B_{1 x}$ e $\omega_{1 y}^{i}=\gamma_{i} B_{1 y}$. 
O estado inicial do sistema é dado pelo operador densidade de equilíbrio (2.5), sendo que para um intervalo de tempo infinitesimal $d t$ a equação de Liouville-von Neumann fornece o seguinte resultado:

$$
\Delta \rho(d t)=\Delta \rho(0)-\frac{i}{\hbar} \sum_{i=1}^{N}\left[\mathrm{H}(0), \epsilon_{i} \mathrm{I}_{z}^{i}\right] d t
$$

Como o comutador entre operadores $\mathrm{I}_{\alpha}^{i}, \alpha=x, y, z$, é proporcional somente a operadores $\mathrm{I}_{\alpha}^{i}$ e esses operadores não expandem o espaço de operadores hermitianos para mais que 1 spin 1/2, não é possível obter os estados mais gerais possíveis para $\Delta \rho$. Portanto, são necessárias outras interações além do campo principal e do campo de rf para que operações lógicas gerais sejam realizadas. A interação de rf é tratada com mais detalhes na sub-seção 2.1.3.

\section{Interação de Deslocamento Químico}

A primeira interação responsável pela quebra de degenerescência dos níveis de energia Zeeman em um sistema homonuclear ${ }^{4}$ é a interação de Deslocamento Químico $(D Q)$. Embora considere-se o campo magnético principal como sendo espacialmente bastante uniforme, existem contribuições paramagnéticas e diamagnéticas dos elétrons orbitais que se somam a esse campo. Dessa forma, núcleos localizados em diferentes ambientes químicos de uma mesma molécula são submetidos a campos magnéticos distintos. A contribuição $\mathbf{B}_{\mathbf{D Q}}$ ao campo local depende da intensidade e da orientação relativa entre a molécula e o campo principal. Sendo essa dependência linear, tem-se que a forma mais geral é dada pelo produto com um tensor constante de $2^{a}$ ordem:

$$
\mathbf{B}_{\mathbf{D Q}}=-\stackrel{\leftrightarrow}{\sigma} \cdot \mathbf{B}_{\mathbf{0}}
$$

A variável $\stackrel{\leftrightarrow}{\sigma}$ é chamada de tensor de deslocamento químico. Assim, a hamiltoniana livre para um sistema de $N$ spins torna-se:

$$
\mathrm{H}=-\hbar \gamma_{i} \sum_{i=1}^{N} \mathbf{I}^{i} \cdot\left(\mathbb{1}_{i}-\stackrel{\leftrightarrow}{\sigma}_{i}\right) \cdot \mathbf{B}_{\mathbf{0}}=-\hbar \sum_{i=1}^{N} \omega_{0}^{i}\left[\left(1-\sigma_{z z}^{i}\right) \mathbf{I}_{z}^{i}-\sigma_{x z}^{i} \mathbf{I}_{x}^{i}-\sigma_{y z}^{i} \mathbf{I}_{y}^{i}\right]
$$

A intensidade do campo $\mathbf{B}_{\mathbf{D Q}}$ é bem menor que o campo principal $\mathbf{B}_{\mathbf{0}}$ o que implica que as componentes proporcionais aos elementos de $\stackrel{\leftrightarrow}{\sigma}$ em 2.24 podem ser tratadas como perturbações da hamiltoniana principal $-\hbar \sum_{i} \omega_{0}^{i} I_{z}^{i}$.

\footnotetext{
${ }^{4}$ Núcleos de mesma espécie, ou seja, mesmo $\gamma$.
} 
Portanto, considerando-se os termos perturbativos de $1^{a}$ ordem, deve-se considerar somente as contribuições que comutam ${ }^{5}$ com a hamiltoniana principal:

$$
\mathrm{H}=-\hbar \sum_{i=1}^{N} \omega_{0}^{i}\left(1-\sigma_{z z}^{i}\right) \mathrm{I}_{z}^{i}
$$

Conforme comentado, o valor de $\sigma_{z z}^{i}$ depende da orientação da molécula com relação a $\mathbf{B}_{\mathbf{0}}$. Para átomos não paramagnéticos o tensor $\stackrel{\leftrightarrow}{\sigma}$ é simétrico [32], fazendo com que $\sigma_{z z}$ possua a seguinte forma:

$$
\begin{gathered}
\sigma_{z z}=\sigma_{i s o}+\frac{\delta}{2}\left[\left(3 \cos ^{2} \theta-1\right)-\eta \operatorname{sen}^{2} \theta \cos (2 \phi)\right] \\
\sigma_{i s o}=\frac{1}{3}\left(\sigma_{z}^{p}+\sigma_{x}^{p}+\sigma_{y}^{p}\right) \\
\eta=\left(\sigma_{y}^{p}-\sigma_{x}^{p}\right) / \sigma_{z}^{p} \\
\delta=\sigma_{z}^{p} .
\end{gathered}
$$

Os ângulos $\theta$ e $\phi$ são respectivamente os ângulos polar e azimutal que o eixo $z$ do Sistema de Eixos Principais ( $S E P$ ) faz com a direção do campo $\mathbf{B}_{\mathbf{0}}$. O SEP é o sistema de coordenadas em que o tensor $\stackrel{\leftrightarrow}{\sigma}$ é diagonal. O índice super-escrito $p$ em 2.26 refere-se às componentes de $\stackrel{\leftrightarrow}{\sigma}$ nesse sistema de coordenadas. Integrando 2.26 em todo o ângulo sólido encontra-se:

$$
\int_{0}^{2 \pi} d \phi \int_{0}^{\pi} \sigma_{z z} \operatorname{sen} \theta d \theta=4 \pi \sigma_{i s o}
$$

Assim, em sistemas líquidos, em que o movimento Browniano molecular é bastante intenso, somente a componente isotrópica $\sigma_{i s o}$, independente da orientação, se mantém, enquanto que para sistemas sólidos amorfos ocorre uma dispersão de valores de $\sigma_{z z}$ devido às várias orientações presentes na amostra. O resultado desse efeito é evidenciado pelo espectro de pó característico da interação de DQ, em que o alargamento espectral resultante pode causar a sobreposição das linhas espectrais. Uma das maneiras de resolver esse problema em sistemas sólidos é rodar a amostra em torno do ânguld ${ }^{6}$ que anula a componente entre colchetes em $\sigma_{z z}$ na Equação 2.26. Dessa forma, para velocidades de rotação grandes o suficiente, é possível anular a componente anisotrópica de $\sigma_{z z}$ e obter uma dispersão de freqüência bem menor em torno de $\sigma_{i s o}$. Em líquidos, é comum referir-se à frequiência de Larmor de cada núcleo $i$ já incluindo-se o deslocamento químico isotrópico, ou seja, $\omega_{0}^{i}=\gamma_{i} B_{0}\left(1-\sigma_{i s o}^{i}\right)$.

\footnotetext{
${ }^{5}$ Essas contribuições são chamadas de seculares.

${ }^{6}$ Chamado de ângulo mágico e aproximadamente igual a $54,7^{\circ}$ nesse caso.
} 


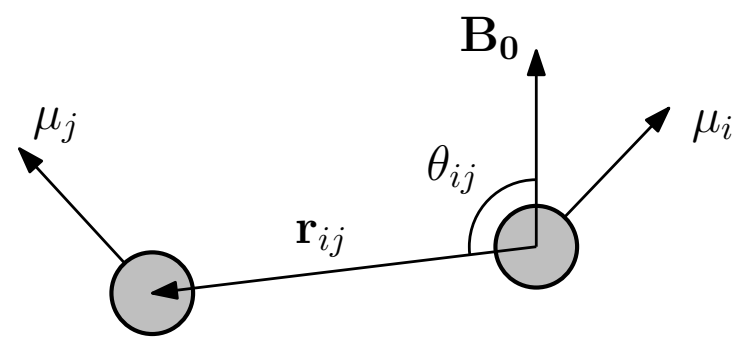

Figura 5: Acoplamento dipolar magnético direto entre dois núcleos. A Equação (2.29) descreve a hamiltoniana dessa interação.

\section{Interação Dipolar Magnética}

A interação mais intensa entre um conjunto de dipolos magnéticos nucleares em uma molécula é a interação dipolar direta, dada pela energia potencial magnética entre todos os pares de dipolos:

$$
E_{d}=\frac{\mu_{0}}{4 \pi} \sum_{i<j}\left\{\frac{\mu_{i} \cdot \mu_{j}}{\left|r_{i j}\right|^{3}}-3 \frac{\left(\mu_{i} \cdot \mathbf{r}_{i j}\right)\left(\mu_{j} \cdot \mathbf{r}_{i j}\right)}{\left|r_{i j}\right|^{5}}\right\}
$$

A hamiltoniana correspondente é dada por:

$$
\begin{gathered}
\mathrm{H}_{d}=\frac{\mu_{0}}{4 \pi} \hbar^{2} \sum_{i<j}\left\{\frac{\gamma_{i} \gamma_{j}}{\left|r_{i j}\right|^{3}}\left(\mathrm{~A}_{i j}+\mathrm{B}_{i j}+\ldots\right)\right\} \\
\mathrm{A}_{i j}=-\mathrm{I}_{z}^{i} \mathrm{I}_{z}^{j}\left(3 \cos ^{2} \theta_{i j}-1\right) \\
\mathrm{B}_{i j}=\frac{1}{2}\left(\mathrm{I}_{x}^{i} \mathrm{I}_{x}^{j}+\mathrm{I}_{y}^{i} \mathrm{I}_{y}^{j}\right)\left(3 \cos ^{2} \theta_{i j}-1\right)
\end{gathered}
$$

Em que somente as duas primeiras contribuições foram explicitadas. Conforme indicado na Figura 5, $\mathbf{r}_{i j}$ é o vetor internuclear e $\theta_{i j}$ é o ângulo que $\mathbf{r}_{i j}$ faz com a direção do campo magnético principal. Aqui também, devido ao fato da intensidade do acoplamento dipolar ser bem menor que a energia Zeeman, somente as contribuições seculares $\mathrm{A}_{i j}$ e $\mathrm{B}_{i j}$ são consideradas na hamiltoniana $\mathrm{H}_{d}$. Na verdade, somente em sistemas homonucleares os termos $\mathrm{B}_{i j}$ comutam com a hamiltoniana principal7. Para o caso heteronuclear é necessário considerar somente o termo $\mathrm{A}_{i j}$. Assim, para um sistema geral contendo várias espécies nucleares pode-se dividir $\mathrm{H}_{d}$ nas contribuições homo, $\mathrm{H}_{o}$, e heteronuclear, $\mathrm{H}_{e}$ :

$$
\begin{gathered}
\mathrm{H}_{d}=\sum_{i \sim j} \mathrm{H}_{o}^{i j}+\sum_{i \ngtr j} \mathrm{H}_{e}^{i j} \\
\mathrm{H}_{o}^{i j}=c_{i j} 2 \pi \hbar\left(3 \mathrm{I}_{z}^{i} \mathrm{I}_{z}^{j}-\overrightarrow{\mathrm{I}}^{i} \cdot \overrightarrow{\mathrm{I}}^{j}\right) \\
\mathrm{H}_{e}^{i j}=c_{i j} 2 \pi \hbar\left(2 \mathrm{I}_{z}^{i} \mathrm{I}_{z}^{j}\right) .
\end{gathered}
$$

\footnotetext{
${ }^{7}$ Vale notar que a hamiltoniana principal é considerada sem o termo de DQ, o qual deve continuar sendo tratado como uma perturbação.
} 
a)

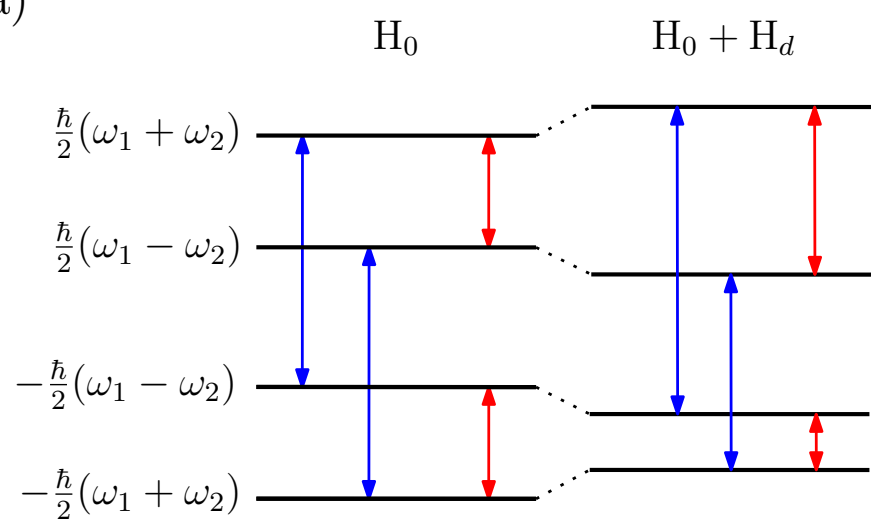

b)

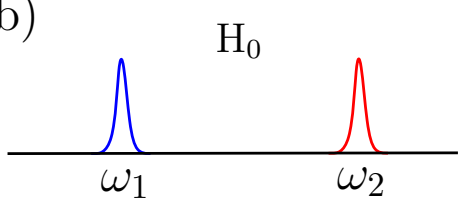

$$
\mathrm{H}_{0}+\mathrm{H}_{d}
$$

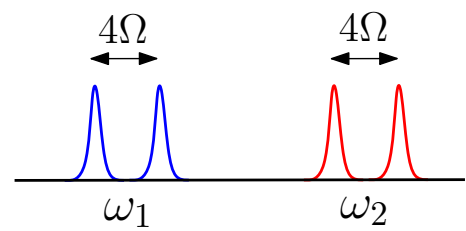

Figura 6: a) Níveis de energia e transições permitidas para dois spins $1 / 2$ heteronucleares acoplados. As transições correspondem aos elementos de matiz não nulos do operador $\mathrm{I}_{+}$, vide sub-seção 2.1.4. b) Linhas espectrais correspondentes às transições permitidas. A presença do acoplamento dipolar permite a distinção de todas as transições. $\omega_{1}=\gamma_{1} B_{0}\left(1-\sigma_{z z}^{1}\right)$ e $\omega_{2}=$ $\gamma_{2} B_{0}\left(1-\sigma_{z z}^{2}\right)$.

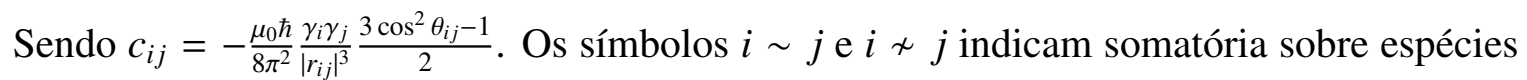
homonucleares e heteronucleares respectivamente. O efeito causado pela interação $\mathrm{H}_{d}$ é o desdobramento das energias Zeeman em estruturas de multipletos. A Figura 6 ilustra essa característica para 2 spins $1 / 2$ heteronucleares.

Da mesma forma que para a interação de DQ, amostras sólidas amorfas produzem um espectro de pó característico. No entanto, observando-se a dependência angular em $\mathrm{H}_{d}$ percebese que o mesmo não possui nenhuma contribuição isotrópica, fazendo com que uma rotação em torno do ângulo mágico, por exemplo, cause o total desacoplamento entre os núcleos do sistema. Isso não é interessante, pois é justamente essa interação entre dois corpos que permite a realização das operações lógicas gerais necessárias para a implementação de CQ. Uma maneira de amenizar esse problema é através da aplicação de sequiências de pulsos de rf que permitem o controle da intensidade dos acoplamentos dipolares ao longo do tempo. Esse controle não só é útil para diminuir os efeitos de descoerência, mas também para viabilizar a construção das portas lógicas de uma forma analítica. Essa última questão é tratada na sub-seção seguinte.

De acordo com a discussão acima, vê-se que a interação dipolar direta não é facilmente observável em sistemas líquidos pois, por não possuir componente isotrópica, o movimento molecular causa o amortecimento dessa interação. 


\section{Interação Dipolar Indireta ou Acoplamento Escalar}

Para sistemas nucleares intra-moleculares existe uma outra interação entre dois dipolos nucleares que se dá indiretamente através dos elétrons orbitais. Essa interação é conhecida por acoplamento escalar ou acoplamento $J$ e possui intensidade bastante inferior ao acoplamento dipolar direto da sub-seção anterior. A forma da hamiltoniana dessa interação é dada por:

$$
\mathrm{H}_{J}=2 \pi \hbar \sum_{i \neq j} J_{i j} \mathbf{I}_{i} \cdot \mathbf{I}_{j}
$$

em que $J_{i j}$ é a intensidade do acoplamento escalar entre o par $i j$. A hamiltoniana (2.31) já é a parte isotrópica desse acoplamento, não possuindo dependência angular, e portanto não sendo anulada em sistemas líquidos. Poderia-se pensar nessa interação como sendo uma possibilidade para o acoplamento entre dois núcleos em um sistema sólido também. No entanto, como a magnitude das principais interações nos sólidos é bem maior que a interação $J$, mesmo usando técnicas de desacoplamento dipolar, não é uma tarefa fácil obter resolução suficiente para a observação desse acoplamento. Enquanto a intensidade do acoplamento direto depende do inverso da distância cúbica entre os núcleos, o acoplamento $J$ depende dos elétrons orbitais compartilhados pelas ligações químicas entre os respectivos átomos.

Para esse caso também é necessário considerar-se quais são as componentes da hamiltoniana de interação que contribuem em perturbação de $1^{a}$ ordem para a hamiltoniana principal. Pode-se mostrar que, semelhantemente ao acoplamento dipolar direto, todos os termos homonucleares de 2.31 comutam com $\mathrm{H}_{0}$. No entanto, devido ao baixo valor de $J$ é comum encontrar-se a seguinte condição:

$$
\left|2 \pi J_{i j}\right| \ll \gamma B_{0}\left|\sigma_{i s o}^{i}-\sigma_{i s o}^{j}\right|
$$

Sob essa condição, chamada de acoplamento fraco, pode-se considerar a interação $J$ como sendo uma perturbação da interação de DQ. Como o termo em $z$ na Equação (2.31) é o único que comuta com a hamiltoniana $\mathrm{DQ}$, somente esse precisa ser considerado para o caso em que se satisfaça a relação 2.32]. Assim, encontra-se a seguinte hamiltoniana $J$ para o sistema:

$$
\begin{gathered}
\mathrm{H}_{J}=\sum_{i \sim j} \mathrm{H}_{o}^{i j}+\sum_{i \ngtr j} \mathrm{H}_{e}^{i j} \\
\mathrm{H}_{o}^{i j}=2 \pi \hbar J_{i j} \mathbf{I}^{i} \cdot \mathbf{I}^{j} \\
\mathrm{H}_{e}^{i j}=2 \pi \hbar J_{i j} 2 \mathrm{I}_{z}^{i} \mathrm{I}_{z}^{j},
\end{gathered}
$$

em que agora o símbolo $i \sim j$ indica espécies homonucleares com acoplamento forte $\mathrm{e} i \nsim j$ indica espécies homonucleares com acoplamento fraco e espécies heteronucleares. 


\section{Interação Quadrupolar}

Como os sistemas quadrupolares são o alvo principal do trabalho apresentado nesta tese, será apresentada nesta sub-seção uma dedução um pouco mais detalhada sobre a forma dessa hamiltoniana. Ainda assim, trata-se de um resumo dos desenvolvimentos que podem ser encontrados nas principais obras de referência [30,33]. Além disso, a dedução que segue será útil na introdução aos operadores tensoriais irredutíveis que serão usados também no método de tomografia exposto no Capítulo 3 .

A interação quadrupolar surge da interação entre o momento de quadrupolo elétrico nuclear e o gradiente de campo elétrico devido à distribuição de cargas eletrônica. Uma maneira de obter a hamiltoniana responsável por essa interação é escrevendo a energia potencial elétrica clássica entre o núcleo e os elétrons e a partir disso obter o respectivo operador quântico. Assim, considerando as densidades de carga do núcleo $\rho_{n}(r)$ e dos elétrons $\rho_{e}(r)$ tem-se que:

$$
E=\iint \frac{\rho_{e}\left(r_{e}\right) \rho_{n}\left(r_{n}\right)}{\left|\mathbf{r}_{n}-\mathbf{r}_{e}\right|} d^{3} r_{e} d^{3} r_{n}
$$

O denominador pode ser substituído pela seguinte expansão em harmônicos esféricos:

$$
\frac{1}{\left|\mathbf{r}_{n}-\mathbf{r}_{e}\right|}=4 \pi \sum_{l=0}^{\infty} \sum_{m=-l}^{l} \frac{1}{2 l+1} \frac{r_{<}^{l}}{r_{>}^{l+1}} Y_{l}^{m}\left(\theta_{n}, \phi_{n}\right) Y_{l}^{m *}\left(\theta_{e}, \phi_{e}\right) \text {. }
$$

Em que $r_{<}$e $r_{>}$significam, respectivamente, o menor e o maior valor entre $r_{e}$ e $r_{n}$. Considerando que a sobreposição da distribuição eletrônica na região do núcleo é pequena, pode-se assumir em todo o espaço que $r_{e}>r_{n}$, obtendo-se:

$$
\begin{gathered}
E=\sum_{l, m} A_{l}^{m} B_{l}^{m *} \\
A_{l}^{m}=\sqrt{\frac{4 \pi}{2 l+1}} \int \rho_{n}\left(r_{n}\right) r_{n}^{l} Y_{l}^{m}\left(\theta_{n}, \phi_{n}\right) d^{3} r_{n} \\
B_{l}^{m}=\sqrt{\frac{4 \pi}{2 l+1}} \int \rho_{e}\left(r_{e}\right) r_{e}^{-(l+1)} Y_{l}^{m}\left(\theta_{e}, \phi_{e}\right) d^{3} r_{e}
\end{gathered}
$$

A densidade de carga nuclear pode ser obtida a partir do valor médio do operador densidade de carga:

$$
\rho_{n}\left(r_{n}\right)=\left\langle\psi_{n}\left|\sum_{i=1}^{A} q_{i} \delta\left(\mathbf{r}_{n}-\mathbf{R}_{i}\right)\right| \psi_{n}\right\rangle
$$

$\psi_{n}\left(\mathbf{R}_{1}, \ldots, \mathbf{R}_{A}\right)$ é a função de onda do núcleo em função das coordenadas $\mathbf{R}_{i}$ de cada nucleon. $A$ é o número de massa, $q_{i}=e$ para os prótons e $q_{i}=0$ para os nêutrons. 
Substituindo-se 2.37) em 2.36), pode-se escrever o termo $A_{l}^{m}$ como sendo o valor médio do operador $\mathrm{A}_{l}^{m}$ :

$$
\mathrm{A}_{l}^{m}=\sqrt{\frac{4 \pi}{2 l+1}} \sum_{i=1}^{A} e_{i} R_{i}^{l} Y_{l}^{m}\left(\Theta_{i}, \Phi_{i}\right),
$$

em que $R_{i}, \Theta_{i}$ e $\Phi_{i}$ são as coordenadas polares de cada nucleon. Analogamente, obtém-se para $\mathrm{B}_{l}^{m}$ a expressão:

$$
\mathrm{B}_{l}^{m}=-e \sqrt{\frac{4 \pi}{2 l+1}} \sum_{i=1}^{N} r_{i}^{-(l+1)} Y_{l}^{m}\left(\theta_{i}, \phi_{i}\right),
$$

em que $r_{i}, \theta_{i}$ e $\phi_{i}$ são as coordenadas de cada elétron. Dessa forma, obtém-se a seguinte hamiltoniana para a interação eletrostática elétron-núcleo:

$$
\mathrm{H}=\sum_{l, m} \mathrm{~A}_{l}^{m} \mathrm{~B}_{l}^{m *}
$$

Considerando-se primeiramente as variáveis nucleares, tem-se que os operadores $\mathrm{A}_{l}^{m}$ satisfazem a definição de operadores tensoriais irredutíveis. Um Tensor Irredutível $]^{8}(T I)$ de rank $l$ possui $2 l+1$ componentes e transforma-se sob rotações da mesma maneira que os harmônicos esféricos. Portanto, vê-se diretamente da Equação 2.38 que o operador $\mathrm{A}_{l}^{m}$ é um tensor irredutível de rank $l$. O fato desses operadores constituírem-se em tensores irredutíveis torna possível o uso do Teorema de Wigner-Eckart no cálculo dos seus elementos de matriz.

Suponha que se use uma base de auto-vetores $|\alpha, J, M\rangle$ para representar os elementos de matriz do operador irredutível $\mathrm{A}_{l}^{m}$. Em que $J$ corresponde aos auto-valores do momento angular total e $M$ são os auto-valores da projeção do momento angular em algum eixo de quantização. $\alpha$ representa todos os outros auto-valores correspondentes aos operadores que comutam com o operador momento angular, de forma tal que $|\alpha, J, M\rangle$ expanda o espaço de estados do sistema. $\mathrm{O}$ teorema de Wigner-Eckart diz que a dependência dos elementos de matriz de $\mathrm{A}_{l}^{m}$ com a orientação do sistema de coordenadas está inteiramente contida nos coeficientes de ClebschGordan. Ou seja:

$$
\left\langle\alpha^{\prime} J^{\prime} M^{\prime}\left|\mathrm{A}_{l}^{m}\right| \alpha J M\right\rangle=\left\langle J^{\prime} l M^{\prime} m \mid J^{\prime} l J M\right\rangle\left\langle\alpha J\left\|\mathrm{~A}_{l}\right\| \alpha^{\prime} J^{\prime}\right\rangle
$$

Os termos $\left\langle J^{\prime} l M^{\prime} m \mid J^{\prime} l J M\right\rangle=C_{J M l m}^{J^{\prime} M^{\prime}}$ são os coeficientes de Clebsch-Gordan que contêm a informação da orientação do sistema via os números quânticos $M, M^{\prime}$ e $m$. $\left\langle\alpha J\left\|\mathrm{~A}_{l}\right\| \alpha^{\prime} J^{\prime}\right\rangle$ é chamado de elemento de matriz reduzido do conjunto de operadores tensoriais $\mathrm{A}_{l}^{m}$.

\footnotetext{
${ }^{8}$ As propriedades dos tensores irredutíveis são tratadas com maior detalhe no Capítulo 3
} 
Os operadores $\mathrm{A}_{l}^{m}$ também são chamados de momento de multipolo de ordem $l$. Essa denominação se deve ao fato de que as componentes $m$ de ordem $l$ contribuem para o momento de $2 l$-polo da distribuição de carga elétrica do sistema [34]. Assim, considerando um núcleo de spin $I$ em que $J=J^{\prime}=I$, tem-se que das propriedades do coeficiente de Clebsch-Gordan, somente as componentes de rank $l$ que satisfazem:

$$
0 \leq l \leq 2 I
$$

são não nulas. Portanto, para um spin $I=1 / 2$ o momento de dipolo será a contribuição mais alta da expansão, enquanto que para um spin $I=1$ o momento mais alto será o de quadrupolo e assim por diante. No entanto, devido ao fato dos harmônicos esféricos possuírem paridade definida sob operação de inversão, $\mathbf{r} \rightarrow-\mathbf{r} \Rightarrow Y_{l}^{m}(\pi-\theta, \phi+\pi)=(-1)^{l} Y_{l}^{m}(\theta, \phi)$, estados nucleares com paridade definida ${ }^{9}$ implicam em contribuições nulas das componentes $A_{l}^{m} \operatorname{com} l$ ímpar. Assim, como os estados estacionários do núcleo são assumidos como tendo paridade definida [30], as contribuições de dipolo, hexapolo, etc. são nulas. Além disso, considerando-se que o raio $R$ do núcleo é bem menor que o raio $a$ dos elétrons, observa-se da Equação (2.36) que pelo fato da dependência radial ser da forma $(R / a)^{l}$, contribuições de ranks mais altos decrescem muito rapidamente. Por esses motivos, a interação eletrostática mais relevante é a do momento de quadrupolo elétrico nuclear, presente em todas as espécies nucleares com spin maior que 1/2. Devido a isso, núcleos com $I>1 / 2$ também são chamados de núcleos quadrupolares. Portanto, basta considerar o rank $l=2$ na hamiltoniana 2.40 .

É impossível construir um conjunto de tensores irredutíveis de rank $l=2$ utilizando-se as componentes esféricas do operador momento angular $\mathbf{I}$ :

$$
\begin{gathered}
\mathrm{T}_{2}^{ \pm 2}=\frac{\sqrt{6}}{4} \beta\left(\mathrm{I}_{ \pm}\right)^{2} \\
\mathrm{~T}_{2}^{ \pm 1}=\frac{\sqrt{6}}{4} \beta\left(\mathrm{I}_{z} \mathrm{I}_{ \pm}+\mathrm{I}_{ \pm} \mathrm{I}_{z}\right), \\
\mathrm{T}_{2}^{0}=\frac{1}{2} \beta\left[3 \mathrm{I}_{z}^{2}-I(I+1) \mathbb{1}_{2 I+1}\right] .
\end{gathered}
$$

Assim, utilizando-se o Teorema de Wigner-Eckart tem-se que os elementos de matriz de $\mathrm{A}_{2}^{m}$ são proporcionais a $\mathrm{T}_{2}^{m}$. Escolhendo $\beta$ em 2.43 de tal forma que:

$$
\frac{\left\langle I M\left|\mathrm{~A}_{2}^{m}\right| I M^{\prime}\right\rangle}{\left\langle I M\left|\mathrm{~T}_{2}^{m}\right| I M^{\prime}\right\rangle}=\frac{\left\langle I\left\|\mathrm{~A}_{2}\right\| I\right\rangle}{\left\langle I|| \mathrm{T}_{2}|| I\right\rangle}=1,
$$

tem-se que os elementos de matriz dos operadores (2.43) são iguais aos elementos dos operadores $\mathrm{A}_{2}^{m}$.

\footnotetext{
${ }^{9}$ Note que funções de onda com paridade definida implicam necessariamente em densidades com paridade par.
} 
Costuma-se definir a quantidade $e Q / 2$ como sendo o valor médio do operador $\mathrm{A}_{2}^{0}$ no estado $|I I\rangle$ :

$$
e Q=2\left\langle I I\left|\mathrm{~A}_{2}^{0}\right| I I\right\rangle=\beta\left\langle I I\left|\left[3 \mathrm{I}_{z}^{2}-I(I+1) \mathbb{1}_{2 I+1}\right]\right| I I\right\rangle=\beta I(2 I-1)
$$

Isolando $\beta$ e substituindo-o em 2.43 obtém-se para os operadores de momento de quadrupolo nuclear o seguinte resultado:

$$
\begin{gathered}
\mathrm{A}_{2}^{ \pm 2}=\frac{e Q}{I(2 I-1)} \frac{\sqrt{6}}{4}\left(\mathrm{I}_{ \pm}\right)^{2}, \\
\mathrm{~A}_{2}^{ \pm 1}=\frac{e Q}{I(2 I-1)} \frac{\sqrt{6}}{4}\left(\mathrm{I}_{z} \mathrm{I}_{ \pm}+\mathrm{I}_{ \pm} \mathrm{I}_{z}\right), \\
\mathrm{A}_{2}^{0}=\frac{e Q}{I(2 I-1)} \frac{1}{2}\left[3 \mathrm{I}_{z}^{2}-I(I+1) \mathbb{1}_{2 I+1}\right] .
\end{gathered}
$$

Pode-se mostrar que as componentes de rank $l=2$ dos tensores eletrônicos $B_{2}^{m}$ satisfazem as seguintes relações:

$$
\begin{gathered}
\mathrm{B}_{2}^{ \pm 2}=\frac{1}{2 \sqrt{6}}\left(\mathrm{~V}_{x x}-\mathrm{V}_{y y} \pm 2 i \mathrm{~V}_{x y}\right) \\
\mathrm{B}_{2}^{ \pm 1}=\frac{1}{\sqrt{6}}\left(\mathrm{~V}_{x z} \pm i \mathrm{~V}_{y z}\right) \\
\mathrm{B}_{2}^{0}=\frac{1}{2} \mathrm{~V}_{z z}
\end{gathered}
$$

em que $V(\mathbf{r})=\left\langle\psi_{e}|\mathrm{~V}(\mathbf{r})| \psi_{e}\right\rangle$ é o potencial elétrico produzido pelos elétrons:

$$
V(\mathbf{r})=-e \sum_{i=1}^{N} \frac{1}{\left|\mathbf{r}-\mathbf{r}_{i}\right|}
$$

e $\mathrm{V}_{\alpha \beta}=\left(\partial^{2} \mathrm{~V} / \partial \alpha \partial \beta\right)_{r=0} \operatorname{com} \alpha, \beta=x, y, z$. Na maioria das substâncias estudadas em RMN os orbitais eletrônicos não são degenerados [30], fazendo com que os valores médios $V_{\alpha \beta}$ possam substituir os operadores $\mathrm{V}_{\alpha \beta}$ na hamiltoniana (2.40). Portanto, os operadores quânticos que compõem a hamiltoniana quadrupolar são de origem exclusivamente nuclear. Para representar os parâmetros da hamiltoniana final é usado novamente o sistema de eixos principais. Nesse sistema de coordenadas, o tensor simétrico $V_{\alpha \beta}$ é nulo para $\alpha \neq \beta$. Normalmente escolhese o sistema de eixos tal que $\left|V_{z z}\right| \geq\left|V_{x x}\right| \geq\left|V_{y y}\right|$ e definem-se os parâmetros eq $=V_{z z}$ e $\eta=\left(V_{x x}-V_{y y}\right) / V_{z z}$. Com essas escolhas, ao substituir-se 2.47) e 2.46) em 2.40 obtémse:

$$
\mathrm{H}_{Q}=\sum_{m} A_{2}^{m} B_{2}^{-m}=\frac{e^{2} q Q}{4 I(2 I-1)}\left[3 \mathrm{I}_{z}^{2}-I(I+1) \mathbb{1}_{2 I+1}+\frac{\eta}{2}\left(\mathrm{I}_{+}^{2}+\mathrm{I}_{-}^{2}\right)\right] .
$$

Utilizando-se um segundo sistema de referência com o seu eixo $Z$ paralelo ao campo principal e supondo que o eixo $Z$ e o eixo $z$ do SEP façam um ângulo $\theta$ entre si, escolhe-se o plano 
de rotação entre esses dois sistemas como sendo o zOx, de tal forma que:

$$
\mathrm{I}_{z}=\mathrm{I}_{Z} \cos (\theta)+\mathrm{I}_{X} \cos (\theta)
$$

Substituindo (2.50) em 2.49) e assumindo por brevidade a simetria cilíndrica para o gradiente de campo elétrico, ou seja $\eta=0$, encontra-se:

$$
\begin{array}{r}
\mathrm{H}_{Q}=\hbar \frac{\omega_{Q}^{\prime}}{4}\left[\frac{1}{2}\left(3 \cos ^{2} \theta-1\right)\left(3 \mathrm{I}_{Z}^{2}-I(I+1)\right)\right. \\
+\frac{3}{2} \operatorname{sen} \theta \cos \theta\left[\mathrm{I}_{Z}\left(\mathrm{I}_{+}+\mathrm{I}_{-}\right)+\left(\mathrm{I}_{+}+\mathrm{I}_{-}\right) \mathrm{I}_{Z}\right] \\
\left.+\frac{3}{4} \operatorname{sen}^{2} \theta\left(\mathrm{I}_{+}^{2}+\mathrm{I}_{-}^{2}\right)\right],
\end{array}
$$

em que $\mathrm{I}_{ \pm}=\mathrm{I}_{X} \pm \mathrm{I}_{Y}$ e a freqüência quadrupolar é definida como:

$$
\omega_{Q}^{\prime}=\frac{3 e^{2} q Q}{\hbar 2 I(2 I-1)} .
$$

Novamente, para o campo magnético $\mathbf{B}_{\mathbf{0}}$ intenso utilizado nos experimentos de RMN a interação quadrupolar é bem mais fraca que a interação Zeeman, $\left|\omega_{Q}\right| \ll\left|\omega_{0}\right|$, fazendo com que somente os termos perturbativos de $1^{a}$ ordem necessitem ser considerados na hamiltoniana $\mathrm{H}_{0}+$ $\mathrm{H}_{Q}$. Com isso encontra-se a expressão final da hamiltoniana quadrupolar em RMN:

$$
\mathrm{H}_{Q}=\hbar \frac{\omega_{Q}}{6}\left[3 \mathrm{I}_{Z}^{2}-I(I+1) \mathbb{1}_{2 I+1}\right],
$$

sendo $\omega_{Q}=\frac{3}{4} \omega_{Q}^{\prime}\left(3 \cos ^{2} \theta-1\right)$. Da mesma forma que para a interação dipolar magnética, a hamiltoniana quadrupolar não possui contribuição isotrópica, não podendo portanto ser observada em sistemas líquidos isotrópicos. Para sólidos amorfos ou policristalinos haverá um espectro de pó característico. Para sólidos monocristalinos, dependendo da intensidade de $\omega_{Q}$, é possível observar o desdobramento das linhas espectrais causado pelo espaçamento desigual dos níveis energéticos na presença da interação quadrupolar. A Figura 4 ilustra esse efeito. Em especial, núcleos quadrupolares imersos em uma matriz líquido-cristalina podem presenciar um gradiente de campo razoavelmente uniforme em toda a amostra, fazendo com que as linhas quadrupolares sejam razoavelmente estreitas. Além disso, devido ao fato dos núcleos possuírem uma razoável mobilidade nesses sistemas, outras interações como a dipolar magnética podem ser atenuadas também, contribuindo ainda mais para o estreitamento das linhas espectrais.

\subsubsection{Pulsos de Radiofreqüência e Gradientes de Campo Magnético}

Conforme visto na sub-seção anterior, as hamiltonianas de interação interna contribuem com termos de acoplamento internuclear do tipo $\mathbf{I}^{i} \cdot \mathbf{I}^{j}$ e termos não lineares $\mathrm{I}_{z}^{2}$ em núcleos 
quadrupolares. Como essas são perturbações estacionárias que comutam com $\mathrm{H}_{0}$, torna-se necessário aplicar perturbações dependentes do tempo que não comutem com a hamiltoniana estacionária e assim produzir operações unitárias gerais sobre o sistema.

Os pulsos de radiofreqüência introduzidos nas equações (2.20) e 2.21) produzem as perturbações dependentes do tempo que possibilitam o controle da evolução dos estados do sistema ao longo da implementação dos algoritmos quânticos. A técnica de espectroscopia por RMN é utilizada com grande versatilidade no desenvolvimento de seqüências de pulsos que efetuam transformações específicas sobre o sistema de spins. Portanto, muitas técnicas consagradas de RMN são empregadas nos experimentos de CQ. Algumas delas serão mencionadas nesta subseção. Particularmente, a técnica de pulsos fortemente modulados descrita na seção 2.2 foi criada tendo como enfoque os experimentos de CQ por RMN, sendo a técnica empregada para a implementação experimental das operações quânticas realizadas neste trabalho.

Embora em princípio seja possível aplicar campos de rf com a forma geral da Equação 2.20), o maior interesse em tais campos é promover a transição entre os níveis energéticos da hamiltoniana estacionária e assim possibilitar o controle da dinâmica dos estados correspondentes. Para isso, é necessário aplicar perturbações variáveis no tempo com frequiências próximas às respectivas freqüências de transição do sistema:

$$
\mathbf{B}_{1}(t)=B_{1}(t)\{\cos [\omega t+\phi(t)] \hat{\mathbf{x}}+\operatorname{sen}[\omega t+\phi(t)] \hat{\mathbf{y}}\}
$$

A maneira mais precisa de representar o efeito desse campo é descrevendo o operador densidade no sistema giratório de coordenadas com frequiência $\omega$. Essa mudança de coordenadas é obtida pela transformação unitária $\mathrm{U}=\exp \left(-i \omega \mathrm{I}_{z} t\right)$, a qual produz a seguinte hamiltoniana efetiva:

$$
\mathrm{H}^{e f}(t)=-\hbar \Omega \mathrm{I}_{z}+\mathrm{H}_{i n t}-\hbar \omega_{1}(t)\left[\mathrm{I}_{x} \cos \phi(t)+\mathrm{I}_{y} \operatorname{sen} \phi(t)\right]
$$

Em que $\Omega=\omega_{0}-\omega$ e $\mathrm{H}_{\text {int }}$ é a hamiltoniana de interação. Aplicando-se um pulso de freqüência $\omega=\omega_{0}{ }^{10}$, observa-se que somente os termos perturbativos sobrevivem em $\mathrm{H}^{\text {ef }}$, fazendo com que auto-estados de $\mathrm{H}_{0}$ não sejam mais auto-estados de $\mathrm{H}^{e f}$ e produzindo algum tipo de dinâmica. Logo, todos os campos de rf empregados têm a forma 2.54) $\operatorname{com} \omega \approx \omega_{0}$.

Devido à grande variedade de seqüências de pulsos existentes em RMN, pode-se pensar em várias classificações para os tipos de pulsos e sequiências. Um primeiro critério seria a questão da seletividade dos pulsos de rf, cujos extremos de classificação seriam os pulsos seletivos e os não-seletivos. Os pulsos exclusivamente não-seletivos possuem a propriedade de

\footnotetext{
${ }^{10}$ Essa condição é chamada de ressonância.
} 
aplicar rotações globais sobre o sistema de spins. Isso ocorre, pois esses pulsos possuem uma intensidade grande o suficiente para que, no período de sua aplicação, as evoluções devido à hamiltoniana de interação em (2.55) sejam desprezíveis. Nesse caso, a hamiltoniana (2.55) dá origem ao operador rotação, em que o ângulo infinitesimal de nutação é dado por $-\omega_{1}(t) d t$ em torno do eixo que faz um ângulo $\phi(t)$ com o eixo $x$ no plano $x y$ na condição de ressonância. Como os pulsos não-seletivos são usados no processo de tomografia proposto nesta tese, a seção 3.1 trata exclusivamente sobre eles.

A seletividade em um pulso de rf diz respeito à sua capacidade de promover transições específicas entre os níveis energéticos da hamiltoniana estacionária. Assim, um pulso seletivo deve ter a capacidade de produzir, ao menos aproximadamente, a seguinte hamiltoniana média:

$$
\overline{\mathrm{H}}=\mathrm{H}_{c}+\beta \mathrm{I}_{\alpha}^{r s}
$$

A hamiltoniana média é definida como sendo $\exp \left(-\frac{i}{\hbar} \overline{\mathrm{H}} t_{p}\right)=\mathrm{U}\left(t_{p}\right)$. Em que U é o operador evolução no instante $t_{p}$ para a hamiltoniana efetiva exata, sendo obtido da solução da equação de Schrödinger para propagadores:

$$
i \hbar \frac{\partial \mathrm{U}(t)}{\partial t}=\mathrm{H}^{e f}(t) \mathrm{U}(t)
$$

$\mathrm{H}_{c}$ é um termo que comuta com $\mathrm{H}_{0}, \beta$ é uma constante e $\mathrm{I}_{\alpha}^{r s}$ é uma combinação dos operadores de transição seletiva $\mathrm{I}_{x}^{r s}$ e $\mathrm{I}_{y}^{r s}[35,36]$. Esses operadores possuem somente os elementos $(r s)$ e ( $s r$ não nulos, os quais são iguais aos elementos dos operadores $I_{x}$ e $I_{y}$ para um spin $1 / 2$. Por isso, também são chamados de operadores de spin 1/2 fictícios. Portanto, a hamiltoniana 2.56) promove a evolução das populações de $\rho$ entre os níveis $r$ e $s$. A diferença $m$ entre os números quânticos magnéticos dos níveis $r$ e $s$ determina o número de quanta transferidos pelo pulso. Portanto, para $m=1$ tem-se um pulso de quantum único, $m=2$ tem-se quantum duplo e assim sucessivamente.

Utilizando-se os operadores de transição seletiva é possível por exemplo obter a hamiltoniana 2.56 para núcleos quadrupolares isolados na condição em que $\omega_{1} \ll \omega_{Q}$. Um exemplo de aplicação desses pulsos está na construção dos estados pseudo-puros da sub-seção 2.1.1. A transformação U da Equação 2.16 é obtida aplicando-se dois pulsos de quantum único seguidos, cujo efeito resulta em uma transição de quantum duplo.

A questão da seletividade constitui portanto o conceito básico de uma sequiência de pulsos. Em geral, o que se procura com uma determinada sequiência é produzir uma hamiltoniana média específica que em geral é composta por pulsos não-seletivos intercalados por períodos 
de evolução livre de perturbação de rf. A maneira analítica comumente utilizada para obter um determinado $\overline{\mathrm{H}}$ é utilizando-se a Teoria da hamiltoniana Média (THM). Para descrevê-la é necessário utilizar a chamada Expansão de Magnus para $\bar{H}$. Essa expansão pode ser obtida dividindo-se a seqüência de pulsos em vários segmentos em que $\mathrm{H}^{e f}$ é independente do tempq ${ }^{11}$. Seja portanto:

$$
\mathrm{H}^{e f}(t)=\mathrm{H}_{k} \quad \text { para } \quad \sum_{i=0}^{k-1} \tau_{i} \leq t<\sum_{i=0}^{k} \tau_{i}
$$

Sendo que $\sum_{i=0}^{n} \tau_{i}=t_{p}$. Aplicando-se a relação de Baker-Campbell-Hausdorff [33]:

$$
e^{\mathrm{B}} e^{\mathrm{A}}=\exp \left\{\mathrm{A}+\mathrm{B}+\frac{1}{2}[\mathrm{~B}, \mathrm{~A}]+\frac{1}{12}([\mathrm{~B},[\mathrm{~B}, \mathrm{~A}]]+[[\mathrm{B}, \mathrm{A}], \mathrm{A}])+\ldots\right\}
$$

sucessivamente sobre o operador evolução $\mathrm{U}\left(t_{p}\right)=e^{-\frac{i}{\hbar} \mathrm{H}_{n} \tau_{n}} \ldots e^{-\frac{i}{\hbar} \mathrm{H}_{0} \tau_{0}}$, obtém-se a hamiltoniana média:

$$
\overline{\mathrm{H}}=\overline{\mathrm{H}}^{(0)}+\overline{\mathrm{H}}^{(1)}+\overline{\mathrm{H}}^{(2)}+\ldots
$$

Em que os dois primeiros termos correspondem a:

$$
\begin{gathered}
\overline{\mathrm{H}}^{(0)}=\frac{1}{t_{p}}\left(\mathrm{H}_{0} \tau_{0}+\ldots+\mathrm{H}_{n} \tau_{n}\right) \\
\overline{\mathrm{H}}^{(1)}=-\frac{i}{2 t_{p}}\left(\left[\mathrm{H}_{1} \tau_{1}, \mathrm{H}_{0} \tau_{0}\right]+\left[\mathrm{H}_{2} \tau_{2}, \mathrm{H}_{0} \tau_{0}\right]+\left[\mathrm{H}_{2} \tau_{2}, \mathrm{H}_{1} \tau_{1}\right]+\ldots\right) .
\end{gathered}
$$

Na prática o que se faz é aplicar pulsos não-seletivos $\mathrm{U}_{k}$ seguidos de um período de evolução livre de duração $\tau_{k}$. Assim, a aplicação dos sucessivos propagadores $\mathrm{U}_{k}=e^{-\frac{i}{\hbar} \mathrm{H}_{k} \tau_{k}}$ corresponde a uma sucessiva mudança de base ${ }^{12}$, fazendo com que se obtenha as hamiltonianas transformadas:

$$
\tilde{\mathrm{H}}_{k}=\mathrm{U}_{1}^{\dagger} \ldots \mathrm{U}_{k}^{\dagger} \cdot \mathrm{H}^{e f}(0) \cdot \mathrm{U}_{k} \ldots \mathrm{U}_{1}
$$

Em que $\mathrm{H}^{e f}(0)$ é a hamiltoniana estacionária antes da aplicação dos pulsos. Escolhe-se a sequiência de pulsos tal que $\mathrm{U}_{n} \mathrm{U}_{n-1} \ldots \mathrm{U}_{1}=\mathbb{1}$, fazendo com que a transformação de $\rho$ devido à mudança de base ao final da seqüência seja nula. Portanto, basta aplicar o propagador $\mathrm{U}\left(t_{p}\right)=$ $e^{-\frac{i}{\hbar} \tilde{\mathrm{H}}_{n} \tau_{n}} \ldots e^{-\frac{i}{\hbar} \tilde{\mathrm{H}}_{0} \tau_{0}}$ sobre o operador $\rho$ para obter o estado do sistema após cada período $t_{p}$.

Para o termo de ordem zero na hamiltoniana média (2.60) obtém-se:

$$
\overline{\mathrm{H}}^{(0)}=\frac{1}{t_{p}} \sum_{k=0}^{n} \tau_{k} \tilde{\mathrm{H}}_{k}
$$

\footnotetext{
${ }^{11}$ Para $\mathrm{H}^{e f}$ variável e continuamente diferenciável no tempo pode-se dividir a hamiltoniana em segmentos arbitrariamente curtos.

${ }^{12}$ Essa mudança de base corresponde à mudança de referencial que acompanha a direção de aplicação dos pulsos, o qual é chamado de toggling frame na literatura de RMN.
} 


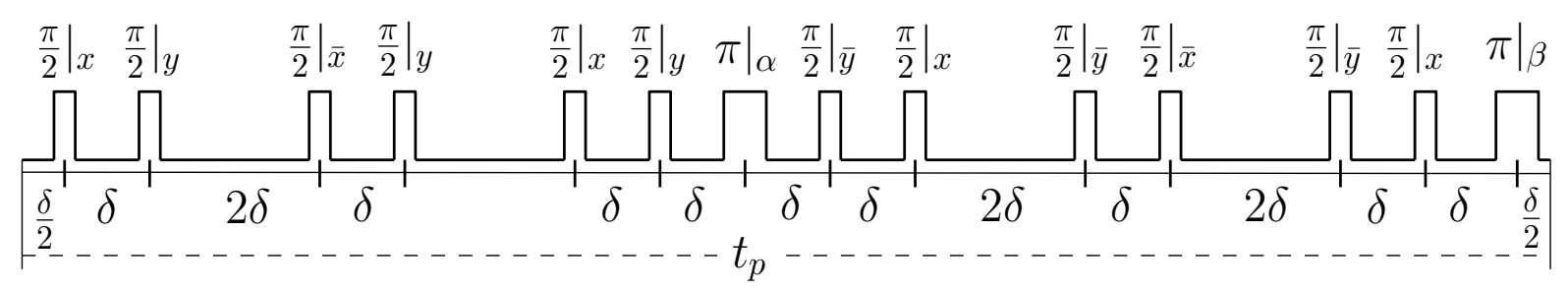

Figura 7: Seqüência de pulsos que produz a hamiltoniana de exchange (2.64) para a ordem mais baixa da expansão de Magnus. Esses são pulsos não-seletivos que promovem a rotação dos spins de ângulos de $\frac{\pi}{2}$ e $\pi$ em torno dos eixos $x, y, \bar{x}=-x$ e $\bar{y}=-y$. Os eixos $\alpha$ e $\beta$ são arbitrários, sendo escolhidos através da fase inicial $\phi$ na equação (2.54). Os pulsos são aplicados nas transições dos dois spins heteronucleares simultaneamente. Seqüência extraída da referência [37].

É fácil verificar que quanto menores os intervalos $\tau_{k}$ mais $\overline{\mathrm{H}}$ se aproxima de $\overline{\mathrm{H}}^{(0)}$. Uma das aplicações da THM está na refocalização(anulamento) das hamiltonianas de interação do sistema. Para citar um exemplo, a seqüência WAHUHA de quatro pulsos possui a propriedade de anular a interação dipolar homonuclear para a hamiltoniana média de ordens 0 e 1 . Um exemplo do uso da THM em CQ está na seqüência ilustrada na Figura 7[38]. Para a ordem zero de $\overline{\mathrm{H}}$ essa seqüência anula todos os acoplamentos dipolares homonucleares e transforma os heteronucleares em:

$$
\overline{\mathrm{H}}^{(0)}=\sum_{i \ngtr j} c_{i j} \frac{4 \pi}{3} \hbar \mathbf{I}^{i} \cdot \mathbf{I}^{j} .
$$

Para dois spins $1 / 2$ essa hamiltoniana produz o operador:

$$
\mathrm{U}=e^{i \frac{\pi}{4}}\left[\begin{array}{llll}
1 & 0 & 0 & 0 \\
0 & 0 & 1 & 0 \\
0 & 1 & 0 & 0 \\
0 & 0 & 0 & 1
\end{array}\right]
$$

o qual corresponde a uma operação SWAP, ou seja, troca de bits dos estados computacionais. Ela também é chamada de operação de spin exchange em RMN.

Por fim, para finalizar esta sub-seção, existe um outro elemento de controle nos experimentos de RMN além dos campos de rf. É possível aplicar gradientes espacialmente constantes de campo magnético sobre a amostra. Esses gradientes podem ser modulados no tempo permitindo a sua aplicação na forma de pulsos. O uso de gradientes em estudos de espectroscopia por RMN tem como principal função a destruição de coerências indesejadas da matriz densidade do sistema. A atuação deles sobre $\rho$ se dá da seguinte forma. 
Um gradiente de campo paralelo ao campo principal produz a seguinte dependência espacial na hamiltoniana do sistema:

$$
\mathrm{H}(z, t)=-\hbar \sum_{k}\left(\omega_{0}^{k}+\gamma_{k} G(t) z\right) \mathrm{I}_{z}^{k}+\mathrm{H}_{i n t}+\mathrm{H}_{r f}(t)
$$

Sendo que $G(t)$ é a intensidade do gradiente em unidades de campo magnético por comprimento. Pode-se dividir o operador $\rho$ do ensemble de spins nucleares em contribuições infinitesimais de planos perpendiculares à direção $z$ :

$$
\rho=\frac{1}{z_{f}-z_{i}} \int_{z_{i}}^{z_{f}} \rho(z) d z .
$$

Em que $z_{i}$ e $z_{f}$ são os limites da dimensão $z$ da amostra. Para um período em que o gradiente é constante no tempo e na ausência de campos de rf, é fácil verificar que os elementos de $\rho(z)$ evoluem da forma:

$$
\langle r|\rho(z, t)| s\rangle=\left\langle r\left|\rho_{0}(t)\right| s\right\rangle \exp \left(i G g_{r s} z t\right)
$$

Sendo $g_{r s}=\sum_{k} \gamma_{k}\left(\left\langle r\left|I_{z}^{k}\right| r\right\rangle-\left\langle s\left|\mathbf{I}_{z}^{k}\right| s\right\rangle\right)$ e $\rho_{0}(t)$ é a matriz densidade evoluída como se não existisse o gradiente aplicado. Por simplicidade, suponha uma amostra de dimensão $d$ com área de seção transversal constante e centrada com o gradiente de campo tal que $z_{i}=-d / 2$ e $z_{f}=d / 2$. Assim, ao substituir-se 2.68) em 2.67) obtém-se facilmente:

$$
\langle r|\rho(t)| s\rangle=\left\langle r\left|\rho_{0}(t)\right| s\right\rangle \operatorname{sinc}\left(\frac{G g_{r s} d}{2} t\right) .
$$

Para um gradiente variável no tempo pode-se dividir a evolução de 2.68) em várias evoluções arbitrariamente curtas em que $G$ é aproximadamente constante. Como conseqüência, chega-se em uma solução análoga à (2.69), com a diferença de que o gradiente $G$ deve ser substituído pelo gradiente médio $\bar{G} \equiv \frac{1}{t} \int_{0}^{t} G\left(t^{\prime}\right) d t^{\prime}$.

A função $\operatorname{sinc}(x) \equiv \operatorname{sen}(x) / x$ tende a zero para $x$ tendendo a infinito. Uma maneira de quantificar a região em que a sinc possui uma amplitude apreciável é através do seu primeiro zero alcançado em $x=\pi$. Portanto, para se produzir um amortecimento significativo da coerência $\rho_{r s}$ é necessário a aplicação de um gradiente com duração superior a $\Delta t \approx 2 \pi / G g_{r s} d$.

Portanto, a intensidade do amortecimento é proporcional ao número de quanta magnéticos correspondentes à transição $r s$, sendo que para transições de quantum zero não há amortecimento. Note que para sistemas homonucleares existirão elementos de quantum zero também fora da diagonal (vide Figura 15, cap. 3), fazendo com que nem todas as coerências de $\rho$ possam ser anuladas exclusivamente por gradientes. 
É possível também fazer a seleção de coerências de quanta específicos através da transferência de coerências via a aplicação de pulsos de rf. Assim, suponha que se aplique um gradiente $G$ ao longo do período $T$. Cada coerência ganhará uma fase adicional proporcional a $g_{r s}:$

$$
\langle r|\rho(z, T)| s\rangle=\left|\left\langle r\left|\rho_{0}(0)\right| s\right\rangle\right| e^{\frac{i}{\hbar} E_{r s} T} e^{i G g_{r s} z T}
$$

Em que $E_{r s}=E_{r}-E_{s}$ é a diferença entre os auto-estados da hamiltoniana estacionária. É possível aplicar um pulso ou uma seqüência de pulsos de rf que produz a transformação geral $\tilde{\rho}_{r s}=\sum_{p q} a_{p q} \rho_{p q}^{*}$, sendo que os operadores densidade sem e com " $\sim$ " correspondem aos instantes imediatamente antes e após o pulso, respectivamente. Após essa transformação, pode-se deixar o estado $\tilde{\rho}$ evoluir por um período $T^{\prime}=\frac{E_{u v}}{E_{r s}} T$ e sob o gradiente $G^{\prime}=\frac{T}{T^{\prime}} \frac{g_{u v}}{g_{r s}} G$, produzindo:

$$
\begin{aligned}
\left\langle r\left|\tilde{\rho}\left(z, T+T^{\prime}\right)\right| s\right\rangle & =\sum_{g_{p q}=g_{u v}} a_{p q}\left|\left\langle p\left|\rho_{0}(0)\right| q\right\rangle\right| \\
& +\sum_{g_{p q} \neq g_{u v}} a_{p q}\left|\left\langle p\left|\rho_{0}(0)\right| q\right\rangle\right| e^{\frac{i}{\hbar}\left(E_{u v}-E_{p q}\right) T} e^{i z G\left(g_{u v}-g_{p q}\right) T} .
\end{aligned}
$$

Da discussão da Equação 2.67) a 2.69 nota-se que para $G$ ou $T$ grandes o suficiente somente a primeira somatória não será anulada e permanecerá inalterada. Logo, com esse experimento é possível selecionar somente as coerências de quantum $g_{u v}$ na posição das coerências de quantum $g_{r s}$. No método de tomografia exposto no Capítulo 3 é utilizado um método de seleção de coerências empregando média temporal ao invés da média espacial via gradientes.

\subsubsection{Leitura de Estados Quânticos}

Conforme mencionado na sub-seção 2.1.1, em RMN são feitas medidas sobre um ensemble de $n$ moléculas computacionais ${ }^{13}$, em que $n$ é um número no mínimo da ordem de centésimos do número de Avogadro. Além disso, cada molécula computacional possui $N$ núcleos computacionais ${ }^{14}$. Para um estado inicial correspondendo ao estado de equilíbrio térmico, o operador densidade do sistema é dado pelo estado produto dos $n \times N$ núcleos, estando cada núcleo no estado (2.14). Assim, após alguma perturbação de rf, o sistema evolui para um estado que em geral não pode mais ser escrito como o produto de cada núcleo.

\footnotetext{
${ }^{13}$ Conjunto de moléculas pré escolhidas na amostra sobre as quais se realizam os experimentos de CQ.

${ }^{14}$ Conjunto de núcleos das moléculas computacionais sobre os quais se realizam os experimentos de CQ.
} 
No entanto, como normalmente as interações intra-moleculares são bem mais intensas que as inter-moleculares é possível, para um tempo $T_{u}$ da ordem do inverso das frequiências das interações intra-moleculares, escrever o operador densidade do sistema como o estado produto:

$$
\rho=\prod_{i=1}^{n} \rho_{i} .
$$

Em que $\rho_{i}$ é o operador densidade de cada molécula no espaço computacional total da amostra. A magnetização medida experimentalmente é, sob certas aproximações, proporcional ao valor médio da componente transversal do momento de dipolo total $\mu=\hbar \sum_{i=1}^{n N} \gamma_{i} \mathrm{I}_{\phi}^{i}$, com $\phi$ igual a $x$ ou $y$ :

$$
\begin{aligned}
M(t) & \propto \operatorname{Tr}\left\{\prod_{i=1}^{n} \rho_{i}(t) \cdot \sum_{j=1}^{n} \mu_{j}\right\}=\operatorname{Tr}\left\{\sum_{j=1}^{n} \rho_{1} \ldots \rho_{j-1} \cdot \rho_{j} \mu_{j} \cdot \rho_{j+1} \ldots \rho_{n}\right\} \\
& =\operatorname{Tr}\left\{\sum_{j=1}^{n} \rho_{j}^{r}(t) \cdot \mu_{r}\right\} \\
& =n \operatorname{Tr}\left\{\bar{\rho}(t) \cdot \mu_{r}\right\} .
\end{aligned}
$$

Na primeira linha, $\mu_{j}$ é o operador momento de dipolo da $j$-ésima molécula computacional escrito no espaço de Hilbert total. Na segunda linha, $\rho_{j}^{r}$ e $\mu_{r}$ são os operadores da $j$-ésima molécula computacional escritos no sub-espaço da respectiva molécula. Por fim, na última linha, $\bar{\rho}=\frac{1}{n} \sum_{i=1}^{n} \rho_{i}^{r}$ é o estado médio sobre todas as moléculas computacionais. Portanto, a medida de RMN fornece o valor médio da componente transversal do momento de dipolo do estado médio da molécula computacional na amostra. Na aproximação de baixa interação intermolecular, toda evolução é restrita ao sub-espaço molecular, sendo portanto evoluções unitárias sobre $\bar{\rho}$. Para tempos superiores a $T_{u}$ ocorre evolução entre sub-espaços distintos, o que acarreta evoluções não unitárias sobre $\bar{\rho}$. Esse efeito é mais evidenciado em amostras sólidas, em que a posição relativa dos diferentes núcleos permanece praticamente constante. Para sistemas líquidos, a interação intermolecular é bastante reduzida devido ao rápido movimento molecular de translação. No entanto, em ambos os casos, interações com campos temporalmente flutuantes, cuja origem se deve aos vários graus de liberdade da amostra, também constituem um processo não unitário. É esse processo que promove a relaxação dos estados do sistema de forma a levá-los novamente ao estado de equilíbrio térmico. Esse assunto é discutido com mais detalhes na seção 2.3 . 
Através da chamada detecção em quadratura é possível medir simultaneamente as duas componentes perpendiculares da magnetização transversal, as quais representadas na forma de fasor resultam em:

$$
\begin{aligned}
M(t) & =M_{x}(t)+i M_{y}(t) \\
& =\operatorname{Tr}\left\{\rho(t) \cdot \sum_{i=1}^{N} \gamma_{i} \mathrm{I}_{x}^{i}\right\}+i \operatorname{Tr}\left\{\rho(t) \cdot \sum_{i=1}^{N} \gamma_{i} \mathrm{I}_{y}^{i}\right\} \\
& =\operatorname{Tr}\left\{\rho(t) \cdot \sum_{i=1}^{N} \gamma_{i}\left(\mathrm{I}_{x}^{i}+i \mathrm{I}_{y}^{i}\right)\right\}=\operatorname{Tr}\left\{\rho(t) \cdot \sum_{i=1}^{N} \gamma_{i} \mathrm{I}_{+}^{i}\right\} .
\end{aligned}
$$

Da Equação (2.74) em diante todos os operadores são assumidos como sendo escritos no espaço de Hilbert da molécula computacional.

O procedimento mais simples de medida de um estado quântico é feito da seguinte maneira. Primeiramente, aplica-se a seqüência de pulsos correspondente às operações unitárias desejadas, levando o estado inicial $\rho_{i}$ ao estado final $\rho_{f}$. A partir desse instante, deixa-se o sistema evoluir sob a hamiltoniana estacionária que produz a transformação $\mathrm{U}(t)=e^{-\frac{i}{\hbar}\left(\mathrm{H}_{0}+\mathrm{H}_{\text {int }}\right) t}$. Ao longo desse período, em que não são aplicadas perturbações de $\mathrm{rf}^{15}$, a magnetização $M(t)$ é medida através do sinal induzido na bobina de detecção:

$$
M(t)=\operatorname{Tr}\left\{\mathrm{U}(t) \rho_{f} \mathrm{U}^{\dagger}(t) \cdot \sum_{i=1}^{N} \gamma_{i} \mathrm{I}_{+}^{i}\right\} .
$$

É fácil mostrar que a Equação 2.75 resulta em:

$$
M(t)=\sum_{i=1}^{N} \gamma_{i} \sum_{r, s} e^{i \omega_{r s} t}\left[\mathrm{I}_{+}^{i}\right]_{r s}\left[\rho_{f}\right]_{r s}^{*},
$$

em que $\omega_{r s}=\left(E_{r}-E_{s}\right) / \hbar$, sendo $E_{i}$ os auto-estados de $\mathrm{H}_{0}+\mathrm{H}_{\text {int }}$. Como o operador $\mathrm{I}_{+}^{i}$ atua somente no sub-espaço do $i$-ésimo núcleo, as freqüências $\omega_{r s}$ são da ordem das freqüências de Larmor $\omega_{0}^{i}$ dos respectivos núcleos. Para espécies nucleares distintas as freqüências $\omega_{0}^{i}$ são bastante diferentes, tornando normalmente necessário ${ }^{16}$ adquirir separadamente o sinal correspondente a cada espécie: $M_{i}(t)=\gamma_{i} \sum_{j \sim i} \sum_{r, s} e^{i \omega_{r s} t}\left[\mathrm{I}_{+}^{j}\right]_{r s}\left[\rho_{f}\right]_{r s}^{*}$, em que o símbolo $j \sim i$ implica em soma sobre núcleos da mesma espécie. A intensidade de cada sinal $M_{i}$ dependerá não somente de $\gamma_{i}$ mas também da resposta do conjunto amostra+detector. Como essa resposta é difícil de ser quantificada, podendo variar sob condições experimentais distintas, é interessante nor-

\footnotetext{
${ }^{15}$ Ao menos nas freqüências detectadas. Vide por exemplo, a técnica de spin locking em que um intenso campo de rf é aplicado na freqüência de outro núcleo [39].

${ }^{16}$ Isso ocorre pois o circuito ressonante tem que ter alto fator de qualidade, o que acarreta em alta seletividade espectral.
} 
malizar as respostas $M_{i}$. Assim, pode-se dividir cada $M_{i}$ por um sinal de referência, como por exemplo o sinal medido sobre o estadd ${ }^{17} \rho_{f}=\sum_{j \sim i} \mathrm{I}_{x}^{j}$. Com esse procedimento, a magnetização total do sistema pode ser expressa por:

$$
M(t)=\sum_{r, s} e^{i \omega_{r s} t}\left[\mathrm{I}_{+}\right]_{r s}\left[\rho_{f}\right]_{r s}^{*}
$$

em que $\mathrm{I}_{+}=\sum_{i=1}^{N} \mathrm{I}_{+}^{i}$. Dessa expressão para a magnetização nota-se que só são obtidos os elementos $r s$ para os quais $\left[\mathrm{I}_{+}\right]_{r s}$ é não nulo. Além disso, entre os elementos não nulos é necessário que todos possuam freqüências distintas caso se queira distinguí-los entre si. Isso é ilustrado nas Figuras 4 e 7 , em que antes de se considerar as hamiltonianas de interação observase um número de transições menor que a quantidade de elementos não nulos dos respectivos operadores $\mathrm{I}_{+}$.

A transformação $\rho_{i} \rightarrow \rho_{f}$ tem que ser realizada em um tempo da ordem de $T_{u}$ para que as operações sejam unitárias no sub-espaço da molécula computacional. No entanto, é interessante que o período de leitura de $M(t)$ seja tão longo quanto houver magnetização transversal presente na amostra. Isso é importante para que haja resolução espectral suficiente para se distinguir as diferentes componentes $e^{i \omega_{r s} t}$ em 2.77). Nesse caso, efeitos de relaxação não podem ser desprezados e a Equação (2.77) precisa ser modificada. A maneira mais precisa de incluir efeitos de relaxação sobre a evolução do operador densidade é via Teoria de Redfield [33], a qual será brevemente comentada na seção 2.3. Uma abordagem fenomenológica mais simples para descrever os efeitos de relaxação em cada elemento $\rho_{r s}$ da matriz densidade é impondo uma taxa de decaimento $-1 / T_{r s}$ fixa para cada elemento. Assim, a equação de Liouville-von Neumann é modificada para:

$$
\frac{\partial \rho_{r s}}{\partial t}=-\frac{i}{\hbar}\left[\mathrm{H}_{0}+\mathrm{H}_{i n t}, \rho\right]_{r s}-\frac{\rho_{r s}-\rho_{r s}^{e q}}{T_{r s}} .
$$

Em que $\rho_{r s}^{e q}$ é o valor do elemento no equilíbrio térmico. Essa equação possui a seguinte solução:

$$
\rho_{r s}(t)= \begin{cases}\rho_{r s}(0) e^{i \omega_{r s} t} e^{-t / T_{r s}} & \text { para } r \neq s \\ \rho_{r s}^{e q}+\left(\rho_{r s}(0)-\rho_{r s}^{e q}\right) e^{-t / T_{r s}} & \text { para } r=s\end{cases}
$$

De uma forma mais geral, os efeitos de relaxação de cada elemento podem ser representados por uma função $f_{r s}(t)$ real com módulo decrescente no tempo. No caso específico da equação diferencial 2.78 tem-se que $f_{r s}(t)=e^{-t / T_{r s}}$.

\footnotetext{
${ }^{17}$ Esse estado pode ser obtido pela aplicação de um pulso de rotação de $\pi / 2$ sobre o estado de equilíbrio, vide sub-seção 2.1.3
} 
Assim, calculando a transformada de Fourier de $M(t)=\sum_{r, s} f_{r s}(t) e^{i \omega_{r s} t}\left[\mathrm{I}_{+}\right]_{r s}\left[\rho_{f}\right]_{r s}^{*}$, que agora contém os efeitos de relaxação devido à longa amostragem, obtém-se:

$$
\begin{aligned}
S(\omega) & =\sum_{r, s}\left[\mathrm{I}_{+}\right]_{r s}\left[\rho_{f}\right]_{r s}^{*} \int_{0}^{\infty} f_{r s}(t) e^{i \omega_{r s} t} e^{-i \omega t} d t \\
& =\sum_{r, s}\left[\mathrm{I}_{+}\right]_{r s}\left[\rho_{f}\right]_{r s}^{*}\left[F_{r s}\left(\omega-\omega_{r s}\right)+i G_{r s}\left(\omega-\omega_{r s}\right)\right]
\end{aligned}
$$

em que $F_{r s}$ e $G_{r s}$ são, respectivamente, as partes real e imaginária da transformada de $f_{r s}(t)$. É fácil observar que as funções $F_{r s}\left(\omega-\omega_{r s}\right)$ e $G_{r s}\left(\omega-\omega_{r s}\right)$ são, respectivamente, simétrica e anti-simétrica com relação ao ponto $\omega_{r s}$. As funções $F_{r s}$ e $G_{r s}$ também são chamadas de componente absortiva e dispersiva. São elas que determinam a forma das linhas espectrais obtidas no processo de medição. Para o caso em que as funções $F_{r s}$ e $G_{r s}$ possuem um forte decaimento com relação às distâncias espectrais $\omega_{r s}$ entre diferentes linhas, não há a sobreposição dessas mesmas linhas espectrais. Esse caso corresponde à situação de alta resolução, em que cada termo da soma em 2.80 pode ser analisado independentemente. Essa condição facilita bastante a medida das amplitudes espectrais e a discriminação da contribuição da relaxação para cada linha. Isso porque, devido à paridade definida de $F_{r s}$ e $G_{r s}$ em torno de $\omega_{r s}$, o módulo de cada linha $r s$ será simétrico com relação à freqüência $\omega_{r s}$. Uma vez que o centro de simetria pode ser facilmente inferido, normalmente coincidindo com o máximo da linha, duas abordagens podem ser utilizadas para a medição da amplitude. Medindo-se a área da linha $r s$ no intervalo $\Delta$ de decaimento em torno de $\omega_{r s}$ obtém-se:

$$
\bar{S}_{r s}=\left[\mathrm{I}_{+}\right]_{r s}\left[\rho_{f}\right]_{r s}^{*} \bar{F}_{r s}(\Delta)
$$

em que $\bar{F}_{r s}(\Delta)=\int_{-\Delta}^{\Delta} F_{r s}(\omega) d \omega$. A outra abordagem é medindo-se a amplitude da linha na posição $\omega_{r s}$, a qual resulta em:

$$
S_{r s}=\left[\mathrm{I}_{+}\right]_{r s}\left[\rho_{f}\right]_{r s}^{*} F_{r s}(0)
$$

Para determinar o valor das contribuições $\bar{F}_{r s}(\Delta)$ ou $F_{r s}(0)$ pode-se utilizar novamente um estado de referência como por exemplo $\rho_{f}=\mathrm{I}_{x}$. Deve-se notar que devido ao fato dos elementos $\left[\rho_{f}\right]_{r s}$ serem em geral complexos, é necessária a medição de ambas as partes real e imaginária do espectro para que se obtenha a informação completa desses elementos. A possibilidade de uma tal medida decorre da detecção em quadratura, que ao ser pós-processada na forma de fasores fornece os resultados acima. A Figura 8 ilustra o processo de medida das linhas espectrais na condição de alta resolução e para o caso de linhas lorentzianas resultantes da Equação 2.79. Portanto, usando-se um estado de referência, as amplitudes medidas podem ser normalizadas de forma tal que correspondam diretamente às componentes $S_{r s}=\left[\mathrm{I}_{+}\right]_{r s}\left[\rho_{f}\right]_{r s}^{*}$ em 2.77 . 


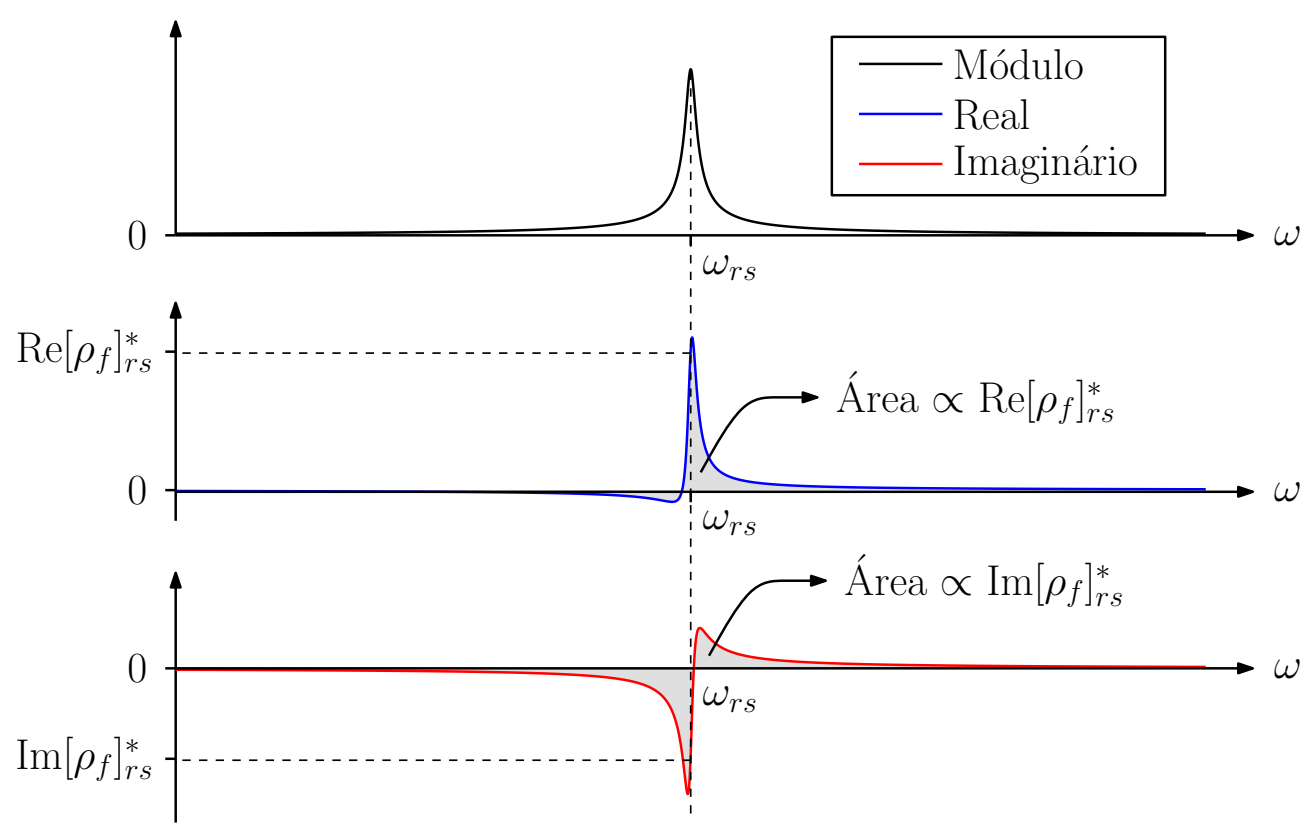

Figura 8: Medida das componentes reais e imaginárias das linhas espectrais na condição de alta resolução. Medindo-se a área ou a amplitude das duas componentes com relação ao centro do módulo da linha obtém-se a informação sobre as partes real e imaginária das coerências de $\rho$.

Caso a largura das formas de linha espectrais não seja suficientemente estreita e ocorra a sobreposição de diferentes linhas, as condições de simetria acima não serão mais satisfeitas e ocorrerá a interferência entre diferentes componentes $r s$. Nesses casos, o procedimento mais geral a ser adotado é fazer um ajuste numérico da equação (2.80) ao espectro medido. Vê-se que nessa situação pode existir a necessidade de se saber a forma das funções de relaxação $f_{r s}$ para que o procedimento de ajuste seja viável.

Com relação aos métodos de criação de estados pseudo-puros discutidos na sub-seção 2.1.1. é fácil observar que devido à linearidade da operação de traço, todos os processos de média entre estados diferentes correspondem à média entre as medidas desses mesmos estados:

$$
\begin{aligned}
\bar{M}(t) & =\frac{1}{M} \sum_{\mu=1}^{M} \operatorname{Tr}\left\{\mathrm{U}(t) \mathrm{U}_{\mu} \rho_{e q} \mathrm{U}_{\mu}^{\dagger} \mathrm{U}^{\dagger}(t) \cdot \mathrm{I}_{+}\right\} \\
& =\operatorname{Tr}\left\{\mathrm{U}(t)\left(\frac{1}{M} \sum_{\mu=1}^{M} \mathrm{U}_{\mu} \rho_{e q} \mathrm{U}_{\mu}^{\dagger}\right) \mathrm{U}^{\dagger}(t) \cdot \mathrm{I}_{+}\right\} . \\
& =\operatorname{Tr}\left\{\mathrm{U}(t) \bar{\rho} \mathrm{U}^{\dagger}(t) \cdot \mathrm{I}_{+}\right\}
\end{aligned}
$$

Assim, nos processos de média temporal é necessário armazenar as diferentes medidas para que a média seja realizada posteriormente. Entretanto, no processo de média espacial via gradientes, como o indicado na Equação (2.67), o valor médio total é obtido simultaneamente. 
Outra característica importante que surge da forma com que o sinal de RMN é medido diz respeito à incapacidade de se obter informação sobre a componente proporcional à identidade nos operadores densidade do sistema. Isso ocorre pois os observáveis de RMN são projeções do operador momento angular I, as quais possuem traço nulo. Portanto, essa característica justifica ainda mais a representação dos estados do sistema em RMN através das matrizes densidade de desvio. Devido a isso, os resultados experimentais e simulações contidos nos capítulos a seguir apresentam somente as matrizes densidade de desvio.

\subsection{SMP's}

Seqüências de pulsos, gradientes de campo e procedimentos de média temporal constituemse nas ferramentas disponíveis para produzir-se experimentalmente por RMN os estados pseudopuros e as portas lógicas utilizadas nos algoritmos quânticos. Procedimentos analíticos como o uso da Teoria da hamiltoniana Média ou a descrição de pulsos seletivos em transições de múltiplos quanta são normalmente utilizados para produzir as hamiltonianas efetivas que correspondem às operações unitárias usadas nos algoritmos de CQ. No entanto, operações quânticas mais gerais nem sempre são obtidas com facilidade utilizando-se procedimentos analíticos. Dessa forma, procedimentos numéricos podem ser úteis para a produção dos resultados desejados. Na verdade, este é um exemplo de problema inverso no qual, conhecendo-se o resultado final almejado, procura-se determinar as condições que levam o sistema a esse resultado. Nesta seção é apresentada uma técnica de otimização numérica denominada Strongly Modulating Pulses (SMP) [15], a qual foi utilizada para implementar experimentalmente as operações quânticas apresentadas nesta tese. Ela é uma variação das técnicas de otimização numérica de pulsos modulados.

Pulsos modulados é o nome genérico dado aos pulsos de rf apresentados na Equação 2.54, em que procura-se encontrar a forma temporal (modulação) das curvas de amplitude $B_{1}(t)$ e de fase $\phi(t)$ para que o sistema de spins sofra uma evolução específica. Eles contrastam com os pulsos retangulares, em que ambos os parâmetros $B_{1}$ e $\phi$ são mantidos constantes ao longo da duração do pulso. O interesse inicial nos pulsos modulados está na promoção de rotações específicas do sistema de spins. Na condição de ressonância, a aplicação de um pulso retangular não-seletivo de duração $\tau$ corresponde a uma rotação de $-\gamma B_{1} \tau$ em torno da direção $\phi$ contida no plano $x y$. No entanto, nem sempre a condição de não-seletividade é satisfatoriamente alcançada e evoluções mais complexas devido à hamiltoniana de interação $\mathrm{H}_{\text {int }}$ podem tornar-se significativas, vide seção 3.1 . Além disso, variações espaciais no valor de $B_{0}$ causam variações do eixo de rotação, que agora possuirá uma componente variável ao longo da direção $z$. Esses 
efeitos obviamente prejudicam o desempenho do pulso de rotação. A forma com que os pulsos modulados podem resolver esse problema é através do conceito de auto-refocalização, em que a atuação conjunta do campo de rf e da hamiltoniana estática levam a magnetização do sistema de spins, após uma evolução arbitrária e usualmente complicada, a uma direção específica e pré-determinada. Portanto, o termo auto-refocalização refere-se ao efeito de anulação ao final do pulso de toda evolução que não corresponda à rotação desejada.

O processo de otimização dos pulsos modulados requer a definição de uma medida de fidelidade e a definição do conjunto de variáveis a serem otimizadas. A escolha das variáveis de otimização depende da forma como os parâmetros do pulso de rf são representados. Como exemplo, pode-se utilizar a expansão em série de Fourier para a amplitude de rf:

$$
B_{1}(t)=C_{0}+\sum_{n=1}^{N}\left[C_{n} \cos \left(\frac{2 \pi n}{\tau} t\right)+S_{n} \operatorname{sen}\left(\frac{2 \pi n}{\tau} t\right)\right] .
$$

Em que $\tau$ é a duração do pulso. Devido a limitações instrumentais, pode ser interessante impor a condição $B_{1}(0)=B_{1}(\tau)=0$ obtida pelo vínculo $C_{0}=-\sum_{n=1}^{N} C_{n}$. Essa mesma expansão pode ser usada para a função $\phi(t)$. Dessa forma, as variáveis $C_{n}$ e $S_{n}$ definem os parâmetros de otimização.

Uma operação útil para a medida de fidelidade é a seguinte generalização do produto escalar:

$$
\mathrm{A} \circ \mathrm{B} \equiv \operatorname{Tr}\left\{\mathrm{A}^{\dagger} \mathrm{B}\right\}
$$

Para o caso em que A e B são vetores, obtém-se o produto escalar usual. A definição 2.85 é bastante utilizada principalmente nos capítulos 3 e 4 desta tese para calcular a projeção de operadores. Fortunato et al. [Fort] utilizam o produto escalar normalizado para definir a projeção $P$ entre o estado teórico almejado, $\rho_{\text {teo }}$, e o estado otimizado numericamente, $\rho_{\text {num }}$ :

$$
P\left(\rho_{\text {teo }}, \rho_{\text {num }}\right) \equiv \frac{\rho_{\text {teo }} \circ \rho_{\text {num }}}{\left|\rho_{\text {teo }}\right|\left|\rho_{\text {num }}\right|}=\frac{\operatorname{Tr}\left\{\rho_{\text {teo }} \rho_{\text {num }}\right\}}{\sqrt{\operatorname{Tr}\left\{\rho_{\text {teo }}^{2}\right\} \operatorname{Tr}\left\{\rho_{\text {num }}^{2}\right\}}} .
$$

Matrizes proporcionais produzem $|P|=1$, definindo matrizes paralelas e anti-paralelas para valores de $P$ iguais a 1 e -1 , respectivamente. Vê-se portanto que a projeção $P$ constitui-se em uma boa medida de fidelidade. Como em geral os algoritmos de otimização procuram minimizar alguma função objetivo, pode-se defini-la como sendo $F=1-P$. Portanto, um valor de $F=0$ $(P=1)$ corresponde a uma otimização perfeita. As rotações da magnetização usualmente buscadas em RMN podem ser obtidas representando-se o estado teórico almejado como sendo a projeção do operador momento angular na direção $\hat{\mathbf{u}}$ de interesse: $\rho_{\text {teo }}=\mathbf{I} \cdot \hat{\mathbf{u}}$. Em aplicações 
de computação quântica os estados teóricos são bastante gerais, uma vez que os estados pseudopuros gerados podem corresponder a uma base arbitrária. Entretanto, o estado de partida para a otimização é sempre o mesmo, correspondendo ao estado de equilíbrio térmico.

Da mesma forma que existe o interesse em otimizar estados específicos, também é possível desenvolver procedimentos de otimização e medidas de fidelidade para operações quânticas realizadas no sistema. Fortunato et al. [15] definem a fidelidade $Q$ de uma operação quântica otimizada numericamente, $\mathrm{U}_{\text {num }}$, com relação a uma operação teórica almejada, $\mathrm{U}_{\text {teo }}$, como sendo a média das projeções das componentes $\mathrm{A}_{i}$ transformadas pelas operações $\mathrm{U}_{\text {num }}$ e $\mathrm{U}_{\text {teo }}$. Em que $\mathrm{A}_{i}, i=1, \ldots, N^{2}$, é qualquer base ortonormal para operadores no espaço de Hilbert $N$-dimensional. Ou seja:

$$
Q\left(\mathrm{U}_{\text {teo }}, \mathrm{U}_{\text {nит }}\right) \equiv \frac{1}{N^{2}} \sum_{i=1}^{N^{2}}\left(\mathrm{U}_{\text {teo }} \mathrm{A}_{i} \mathrm{U}_{\text {teo }}^{\dagger}\right) \circ\left(\mathrm{U}_{\text {num }} \mathrm{A}_{i} \mathrm{U}_{\text {num }}^{\dagger}\right) .
$$

Utilizando a base canônica $\mathrm{e}_{i j},\left[\mathrm{e}_{i j}\right]_{r s}=\delta_{i r} \delta_{j s}$, e expandindo o operador $\mathrm{U}_{\text {teo }}^{\dagger} \mathrm{U}_{\text {num }}$ nessa base, $\mathrm{U}_{\text {teo }}^{\dagger} \mathrm{U}_{\text {num }}=\sum_{r, s} a_{r s} \mathrm{e}_{r s}$, encontra-se para a expressão 2.87):

$$
Q\left(\mathrm{U}_{\text {teo }}, \mathrm{U}_{\text {num }}\right)=\frac{1}{N^{2}} \sum_{i, j, r, s, k, l} \operatorname{Tr}\left\{a_{r s} a_{l k}^{*} \mathrm{e}_{k l} \cdot \mathrm{e}_{j i} \cdot \mathrm{e}_{r s} \cdot \mathrm{e}_{i j}\right\}
$$

Aplicando a relação $\mathrm{e}_{i j} \cdot \mathrm{e}_{r s}=\delta_{j r} \mathrm{e}_{i s}$ em (2.88) chega-se em:

$$
Q\left(\mathrm{U}_{\text {teo }}, \mathrm{U}_{\text {num }}\right)=\frac{1}{N^{2}} \sum_{i, j, k} \operatorname{Tr}\left\{a_{i i} a_{j k}^{*} \mathrm{e}_{k j}\right\}=\frac{1}{N^{2}} \sum_{i} a_{i i} \sum_{j} a_{j j}^{*}=\left|\frac{\operatorname{Tr}\left\{\mathrm{U}_{\text {teo }}^{\dagger} \mathrm{U}_{\text {num }}\right\}}{N}\right|^{2} .
$$

Portanto é possível medir a fidelidade entre as duas operações de forma independente da base utilizada. Uma característica importante do resultado 2.89 ) é que uma fase arbitrária $e^{i \alpha}$ multiplicando o operador $U$ resulta na mesma fidelidade, como é de se esperar da teoria de Mecânica Quântica que diz que vetores de estado que diferem apenas por uma fase global $e^{i \alpha}$ são fisicamente indistinguíveis. Da mesma forma que para a projeção de estados, pode-se definir uma função objetivo que possui o valor zero como sendo a otimização ideal: $F=1-\sqrt{Q}$.

Diferentemente da função de modulação continuamente diferenciável normalmente utilizada empregando-se expansões como a (2.84), Fortunato et al. propõem uma sequiência de transições discretas nos parâmetros do pulso de rf. Entre essas transições, os parâmetros do pulso são mantidos constantes. A denominação SMP é devida a esses saltos descontínuos. 


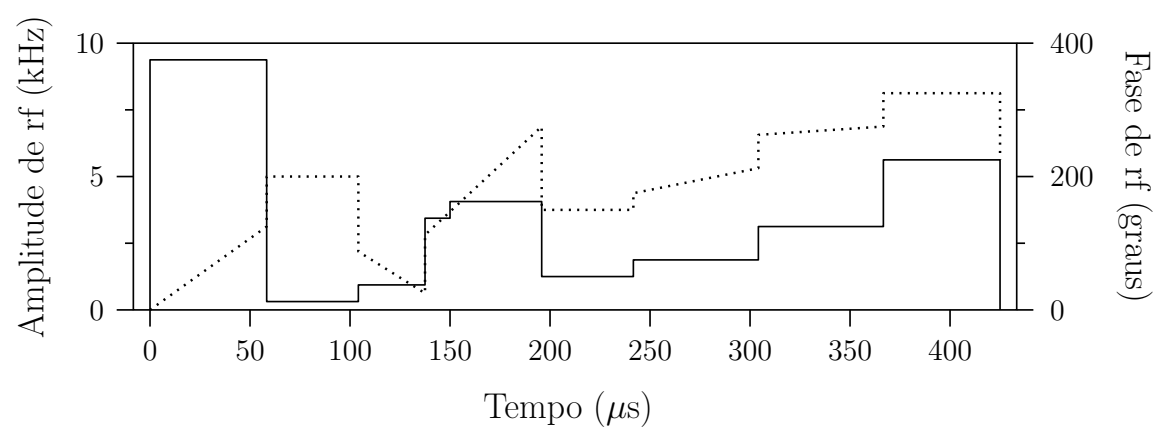

Figura 9: Ilustração da forma de um pulso SMP. Os segmentos cheios representam o valor da amplitude e os segmentos tracejados representam o valor da fase dos pulsos de rf. As fases $\phi_{k}$ correspondem ao valor inicial da reta tracejada em cada segmento. As freqüências $\omega_{k}$ são implementadas pelas rampas de fase. Figura baseada na ref. [15].

Portanto, um pulso SMP é representado da seguinte maneira:

$$
\mathbf{B}_{\mathbf{1}}(t)=B_{1}^{k}\left[\cos \left(\omega_{k} t+\phi_{k}\right) \hat{\mathbf{x}}+\operatorname{sen}\left(\omega_{k} t+\phi_{k}\right) \hat{\mathbf{y}}\right] \quad \text { para } \quad \sum_{i=0}^{k-1} \tau_{i} \leq t<\sum_{i=0}^{k} \tau_{i},
$$

em que $\tau_{0}=0$. O pulso 2.90 está representado no referencial giratório com freqüência próxima a de Larmor. Cada segmento $k$ de um pulso SMP é caracterizado por 4 parâmetros: $B_{1}^{k}$, $\omega_{k}, \phi_{k}$ e $\tau_{k}$. Portanto, para um pulso com $N$ segmentos, o espaço de parâmetros a ser otimizado possui dimensão $4 N$. A Figura 9 ilustra a forma de um pulso SMP.

O tratamento dado aos SMP's nesta seção tem como objetivo os sistemas homonucleares. Para sistemas heteronucleares a equação (2.90) deve conter um conjunto de parâmetros adicional para cada espécie nuclear distinta. O cálculo do efeito de tais pulsos não será feito aqui, sendo que a forma correta de realizar essa descrição é empregando-se o sistema multi-giratório de coordenadas, o qual é discutido na seção 3.1

Descrevendo o operador de cada segmento no referencial em ressonância com a freqüência do respectivo segmento e fazendo a transformação de retorno para o referencial original, podese mostrar que o operador evolução é dado por:

$$
\mathrm{U}_{\text {num }}=\prod_{k=1}^{N} \exp \left(i \omega_{k} \tau_{k} \mathrm{I}_{z}^{k}\right) \exp \left\{i \gamma B_{1}^{k} \tau_{k}\left[\cos \left(\phi_{k}\right) \mathrm{I}_{x}^{k}+\operatorname{sen}\left(\phi_{k}\right) \mathrm{I}_{y}^{k}\right]-\frac{i}{\hbar} \tau_{k} \mathrm{H}_{\text {int }}\right\} .
$$

Devido a limitações do equipamento utilizado para realizar os experimentos expostos neste trabalho (vide seção 4.2) considerou-se mais conveniente não utilizar o parâmetro $\omega_{k}$ como variável de controle no pulso SMP. Mais especificamente, essa escolha se deve ao fato do modulador de pulsos do espectrômetro implementar a freqüência $\omega_{k}$ de cada segmento via uma variação linear da fase, da mesma forma como mostrado nas rampas de fase da Figura 9 . 
No entanto, como os incrementos de fase no modulador são discretos e com tamanho limitado pela resolução temporal, os passos de fase podem divergir do cálculo numérico usando 2.91. Além disso, dos altos valores de fidelidade mostrados nas Tabelas 7, 9 e 11 na seção 3.3. percebe-se que a exclusão do parâmetro $\omega_{k}$ não comprometeu o desempenho das otimizações para o sistema experimental estudado.

Aplicando a Equação (2.91) ao caso do núcleo quadrupolar de spin 3/2 estudado nesta tese, e sem o uso do parâmetro $\omega_{k}$, ou seja, aplicando todos os segmentos em ressonância, obtém-se o seguinte operador evolução:

$$
\mathrm{U}_{\text {num }}=\prod_{k=1}^{N} \exp \left\{i \theta_{k}\left[\cos \left(\phi_{k}\right) \mathrm{I}_{x}+\operatorname{sen}\left(\phi_{k}\right) \mathrm{I}_{y}\right]-i \beta_{k} \mathrm{I}_{z}^{2}\right\},
$$

em que $\theta_{k}=\omega_{1}^{k} \tau_{k}$ e $\beta_{k}=\frac{\omega_{Q}}{2} \tau_{k}$, com $\omega_{1}^{k}=\gamma B_{1}^{k}$. O fato de existir somente um parâmetro que caracteriza a hamiltoniana de interação desse sistema, possibilita a otimização de SMP's de forma independente do valor da constante de interação quadrupolar $\omega_{Q}$. Isso porque mudanças no valor de $\omega_{Q}$ podem ser compensadas alterando-se os valores de $\omega_{1}$ e $\tau$, de forma a manter $\theta$ e $\beta$ inalterados. Essa característica facilita a implementação dos SMP's nesse sistema, pois evita a necessidade de otimização de um novo SMP para cada novo experimento ${ }^{18}$. Note que para uma hamiltoniana de interação caracterizada por dois ou mais acoplamentos não é possível fazer essa compensação, pois os únicos parâmetros ajustáveis da hamiltoniana são sempre os mesmos, ou seja, $\omega_{1}$ e $\tau$. No entanto, na hora de realizar as otimizações é importante utilizar um valor de referência para $\omega_{Q}$ próximo do valor experimental para que a alteração nos valores de $\omega_{1}$ e $\tau$ não ultrapasse os limites instrumentais, vide seção 4.2. Esse procedimento é possível pois, conforme verificou-se experimentalmente, a variação no valor de $\omega_{Q}$ em diferentes experimentos não ultrapassou a faixa de $10 \%$.

Para a otimização de estados pseudo-puros a definição dos parâmetros do pulso é similar à equação 2.92, sendo que agora é necessário especificar um índice a mais para o caso de implementação de pseudo-puros usando média temporal:

$$
\rho_{\text {num }}=\frac{1}{M} \sum_{\mu=1}^{M} \mathrm{U}_{\text {num } \mu} \rho_{e q} \mathrm{U}_{\text {num } \mu}^{\dagger} .
$$

Sendo que os parâmetros de cada segmento são indexados como $\theta_{k}^{\mu}, \phi_{k}^{\mu}$ e $\beta_{k}^{\mu}$. Tendo especificado as medidas de fidelidade e os parâmetros de controle, será exposto agora o algoritmo utilizado no processo de otimização. Neste trabalho foi empregado o algoritmo Simplex Nelder-

\footnotetext{
${ }^{18}$ Para uma mesma amostra de cristal líquido, o acoplamento quadrupolar pode variar com a idade, com a temperatura e até mesmo com o posicionamento da amostra.
} 
a)

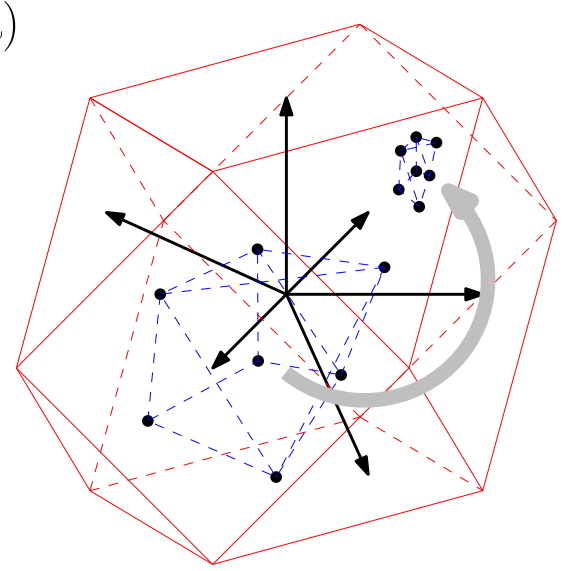

b)

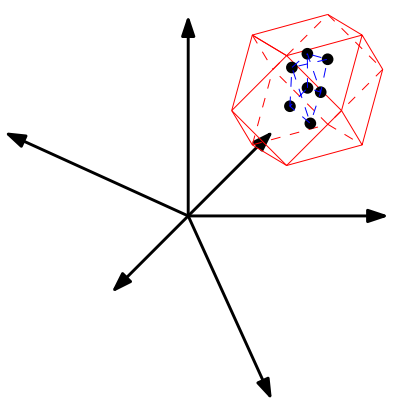

Figura 10: Representação pictórica do processo de otimização no espaço de parâmetros. a) A forma geométrica em vermelho representa o volume de fronteira original. Todas as soluções são buscadas dentro desse volume. Os vetores representam o sistema de eixos perpendiculares entre si no espaço de parâmetros. b) Representação do processo de busca convergente. A fidelidade do simplex azul, formado pelos pontos otimizados, determina o novo volume de busca em vermelho. Esse processo é repetido até algum critério de parada ser alcançado.

Mead [40], o mesmo utilizado por Fortunato et al. [15]. Para descrever o processo de funcionamento desse algoritmo é interessante definir o espaço de parâmetros como sendo o espaço euclidiano de dimensão $3 N$, sendo que cada uma das variáveis $\theta_{k}, \phi_{k}$, e $\beta_{k}, k=1 \ldots N$, corresponde a uma componente ortogonal nesse espaço. Portanto, cada pulso SMP corresponde a um ponto $V \in \mathbb{R}^{3 N}$ no espaço de parâmetros, fazendo com que as funções de fidelidade definidas acima correspondam a um mapa $f: \mathbb{R}^{3 N} \rightarrow \mathbb{R}$. A palavra simplex serve para designar as formas geométricas caracterizadas por $3 N+1$ pontos no espaço de parâmetros. Assim, primeiramente é informado para o algoritmo um simplex inicial, sendo que, na ausência de uma informação mais específica sobre a faixa de parâmetros que contém a solução, pode-se gerar o simplex inicial aleatoriamente. O intervalo de varredura da variável aleatória deve estar limitado pelas restrições experimentais dos parâmetros do pulso. Esse limite corresponde a um volume de fronteira que contém todos os demais simplex no espaço de parâmetros. A Figura 10 a) contém uma ilustração desse procedimento inicial. Uma descrição precisa do funcionamento geral do algoritmo, bem como a descrição da rotina de implementação em linguagem Fortran podem ser obtidas em [41]. Aqui, por motivos didáticos, a descrição se limitará ao caso de um espaço de parâmetros bidimensional. A generalização para uma espaço multi-dimensional pode ser feita sem maiores dificuldades, vide [41].

No caso bidimensional um simplex corresponde a um triângulo, a cujos vértices atribui-se as variáveis $M, I$ e $P$. Para um dado simplex, as variáveis $M, I$ e $P$ são atribuídas respectivamente aos vértices com a $M$ elhor, a Intermediária e a $P$ ior fidelidade entre os três. A primeira etapa é, a partir do simplex inicial, determinar o ponto $R$ que corresponde à $R$ eflexão do ponto 
a)

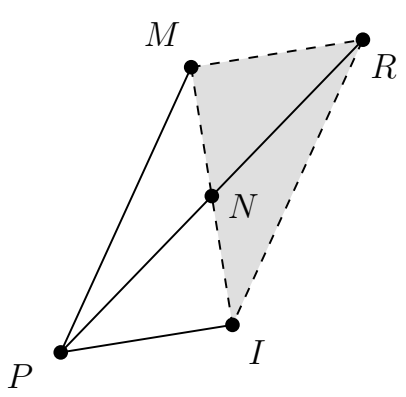

c)

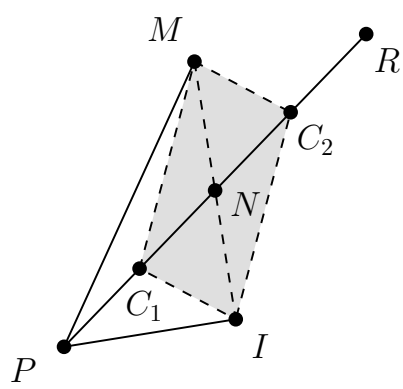

b)

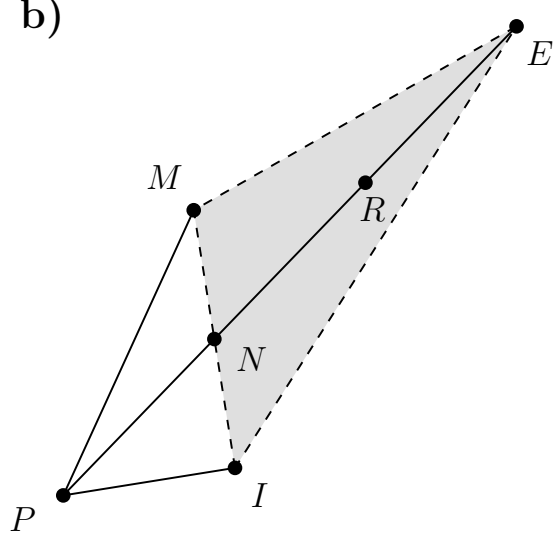

d)

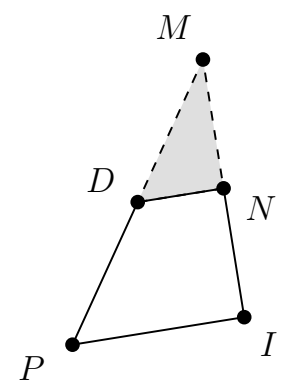

Figura 11: Ilustração do funcionamento do algoritmo de otimização Simplex para um espaço de parâmetros bidimensional. A área em cinza representa a possível atualização do simplex em cada passo do algoritmo.

$P$ com relação à mediaNa $N$, vide Figura 11 a). Caso a fidelidade do ponto $R$ seja melhor que a do ponto $P$ então determina-se o ponto $E$ como sendo a $E$ xtensão do segmento $\overline{N R}$ no sentido oposto a $P$, vide Figura 11 b). Caso a fidelidade de $E$ seja melhor que a de $R$, redefine-se o simplex inicial como sendo constituído pelos pontos $M, I$ e $E$. Caso contrário, redefine-se o simplex inicial como sendo $M, I$ e $R$. No entanto, se a fidelidade de $R$ for ainda pior que a de $P$ então define-se os pontos de $C$ ontração $C_{1}$ e $C_{2}$ como sendo os pontos médios dos segmentos $\overline{P N}$ e $\overline{N R}$ respectivamente, vide Figura 11 c). Se o melhor dos pontos entre $C_{1}$ e $C_{2}$ for melhor que $P$ então o simplex inicial é redefinido como sendo constituído por $M, I$ e o melhor entre $C_{1}$ e $C_{2}$. Caso contrário define-se o ponto de Diminuição $D$ como sendo o ponto médio de $\overline{P M}$, vide Figura 11.d), e redefine-se o simplex inicial como sendo $M, N$ e $D$. Dessa forma estão descritos todos os passos possíveis do algoritmo. A cada novo simplex redefinido deve-se empregar novamente a seqüência descrita desde o início do parágrafo. Utilizando-se algum critério de parada, o algoritmo deve convergir para algum ponto próximo de um mínimo local da função objetivo. 
Para melhorar o processo de busca, de forma a fazer com que o algoritmo encontre valores mais próximos de um mínimo global, foi utilizado um processo de busca convergente baseado no valor de fidelidade obtido após a aplicação do algoritmo Simplex. Esse processo consiste em definir-se uma função $g(F)$ que é estritamente crescente com o valor da função objetivo $F$. Assim, seja $V_{i}=\prod_{n=1}^{3 N} \Delta x_{n}$ o volume de fronteira inicial, em cada que $\Delta x_{n}$ corresponde ao intervalo de busca de cada um dos $3 N$ parâmetros do pulso. Ao aplicar-se o algoritmo descrito no parágrafo anterior sobre o volume $V_{i}$ obtém-se o ponto otimizado $M$. Assim, calcula-se um novo intervalo de busca para cada variável como sendo $\Delta x_{n}^{\prime}=g(F(M)) \Delta x_{n}$. Esse novo intervalo define o novo volume de fronteira centrado em $M$ sobre o qual será aplicado novamente o algoritmo Simplex. A Figura 10 b) ilustra esse processo. Portanto, quanto menor o valor de $F$, menor o próximo volume de busca, o qual é reduzido por um fator $g^{3 N}$. Caso após um número pré-definido de tentativas não se encontre um valor mais baixo de $F$, então volta-se a buscar no volume e fronteira original. A função de convergência adotada na otimização das operações quânticas foi $g(x)=x^{2}$. O Apêndice A.2 contém o código de otimização utilizado para portas lógicas. O código para otimização de estados pseudo-puros não está listado nesta tese por ser bastante similar. A otimização de cada uma dessas operações para o sistema quadrupolar de spin 3/2 demora em torno de 5 minutos rodando em um micro-computador equipado com um processador Pentium IV de 3,2 GHz.

Para terminar esta seção é importante discutir a maneira de incluir as limitações e os erros experimentais no processo de otimização. Primeiramente, é interessante redefinir a função objetivo como sendo a soma com uma função penalidade $P, F^{\prime}=P+F$, a qual assume valores positivos altos para pontos fora do volume de fronteira original e valor zero para pontos dentro desse simplex. Dessa forma, evita-se que o algoritmo busque regiões fora dos limites instrumentais. O procedimento de inclusão de erro pode ser feito calculando-se uma fidelidade média sobre uma distribuição dos parâmetros da hamiltoniana em torno do valor otimizado. Matematicamente, esse processo é escrito como:

$$
\bar{F}=P+\sum_{l, m, n} p_{l m n} F_{l m n}
$$

em que $\sum_{l, m, n} p_{l m n}=1$ é uma distribuição discreta de probabilidades e $P$ é a função penalidade discutida anteriormente. Os índices $l, m, n$ representam o desvio em cada um dos três parâmetros de cada segmento: $\theta_{k}^{l}=\theta_{k}+\Delta \theta_{l}, \phi_{k}^{m}=\phi_{k}+\Delta \phi_{m}$ e $\beta_{k}^{n}=\beta_{k}+\Delta \beta_{n}$. Note que para uma dada trinca de valores de $l, m, n$ o desvio é o mesmo para todos os segmentos, ou seja, é assumido que o erro nos parâmetros é independente do valor dos mesmos. Talvez o procedimento ideal fosse aplicar o algoritmo calculando-se a função objetivo como sendo a função $\bar{F}$. 
No entanto, esse procedimento causa um significativo atraso no tempo de computação do algoritmo. Pode-se perceber isso ao considerar-se uma distribuição pequena de apenas 5 pontos por parâmetro, a qual totaliza 125 pontos para a distribuição. Portanto, para cada rodada do Simplex haveria a necessidade de se computar 125 vezes a função fidelidade, a qual envolve a exponenciação de vários operadores. Portanto, optou-se neste trabalho por calcular a função $\bar{F}$ somente ao final de cada otimização completa, sendo que cada rodada do simplex aplica a função $F^{\prime}$ como função objetivo. Assim, ao final de várias rodadas do algoritmo, escolheu-se a otimização com o maior valor de $\bar{F}$ e com o menor desvio padrão. Com esse procedimento foi possível usar uma distribuição com um grande número de pontos, 10000 no total, e ainda adicionar o erro na freqüência de Larmor através da inclusão do termo $-\hbar \Delta \Omega \mathrm{I}_{z}$ na hamiltoniana do pulso. Os pontos foram gerados aleatoriamente usando-se uma distribuição uniforme sobre o intervalo de erro de cada parâmetro: $\Delta \Omega, \Delta \theta, \Delta \phi$ e $\Delta \beta$. Esses erros relacionam-se com os erros dos parâmetros instrumentais calculando-se as respectivas diferenciais:

$$
\begin{aligned}
& \Delta \Omega=\tau \Delta \omega_{0} \\
& \Delta \theta=\omega_{1} \Delta \tau+\tau \Delta \omega_{1} \\
& \Delta \beta=\frac{\omega_{Q}}{2} \Delta \tau+\frac{\tau}{2} \Delta \omega_{Q}
\end{aligned}
$$

A quantificação de cada um dos erros em 2.95) é realizada na seção 4.2 .

\subsection{Relaxação de Estados Quânticos}

Conforme foi mencionado na sub-seção 2.1.4, para tempos suficientemente longos, evoluções não unitárias no sub-espaço das moléculas computacionais começam a ocorrer. Nesse ponto é possível identificar duas contribuições para o processo de evolução não unitária. A primeira diz respeito aos desvios independentes do tempo na hamiltoniana do sistema. A origem dessa contribuição está na dispersão espacial das constantes de interação da hamiltoniana considerada e das interações externas independentes do tempo que não foram levadas em conta por possuirem baixa intensidade. O resultado dessa contribuição é uma perda de coerência entre os vários estados nucleares, fazendo com que os elementos não diagonais da matriz densidade sejam atenuados com o tempo, de uma maneira análoga à mostrada com o uso de gradientes na seção 2.1. A segunda contribuição corresponde às variações temporalmente aleatórias das hamiltonianas do sistema, que têm como principal origem os movimentos moleculares. Processos como esses são não conservativos e fazem com que o sistema retorne ao estado de equilíbrio térmico. Uma vez que somente a segunda contribuição envolve processos irreversíveis, de fato é ela que contribui genuinamente para a relaxação dos estados quânticos do sistema. 
A maneira de reverter parte das evoluções não unitárias do primeiro tipo é empregando o conceito de seqüências de refocalização. Nessas seqüências procura-se fazer com que em determinados instantes de tempo a hamiltoniana média definida em (2.57) seja nula para a interação escolhida. Em geral é difícil fazer com que a hamiltoniana média de uma determinada interação seja completamente anulada. Por isso é utilizada a expansão de Magnus, em que se procura anular os termos mais relevantes dessa expansão. Exemplos de seqüências de refocalização que fazem uso desse conceito são a WAHUHA mencionada na sub-seção 2.1.2 e a sequiência da Figura 7 que anulam as primeiras ordens da interação dipolar homonuclear. Talvez o exemplo mais simples de uma sequiência de refocalização seja a sequiência de spin-echo, que refocaliza a dispersão da interação Zeeman. Além de sequiências de pulsos, também é usado o procedimento de rotação em torno do ângulo mágico mencionado na sub-seção 2.1.2 para anular interações indesejadas.

O sistema experimental estudado nesta tese é um sistema de núcleos quadrupolares de spin $3 / 2$ isolados. Portanto, serão mostrados aqui alguns exemplos de seqüências de refocalização para esse sistema. Nesse caso, a hamiltoniana estacionária é constituída pela interação Zeeman do campo principal e pela interação quadrupolar:

$$
\mathrm{H}(x)=-\hbar \omega_{0}(x) \mathrm{I}_{z}+\hbar \frac{\omega_{Q}(x)}{6}\left[3 \mathrm{I}_{Z}^{2}-I(I+1) \mathbb{1}_{2 I+1}\right],
$$

em que $\omega_{0}(x)$ e $\omega_{Q}(x)$ contêm os desvios espaciais do campo principal e da interação quadrupolar, respectivamente. Cada elemento $r s$ da matriz densidade em torno da posição $x$ evoluirá da forma: $\rho_{r s}(x, t)=\rho_{r s}(0) e^{i t\left[\mathbf{w}_{0}(x)\right]_{r s}} e^{i t\left[\mathbf{w}_{Q}(x)\right]_{r s}}$, sendo que para o caso de spin $3 / 2$ tem-se:

$$
\mathbf{w}_{0}(x)=\left[\begin{array}{cccc}
0 & -\omega_{0} & -2 \omega_{0} & -3 \omega_{0} \\
\omega_{0} & 0 & -\omega_{0} & -2 \omega_{0} \\
2 \omega_{0} & \omega_{0} & 0 & -\omega_{0} \\
3 \omega_{0} & 2 \omega_{0} & \omega_{0} & 0
\end{array}\right], \mathbf{w}_{Q}(x)=\left[\begin{array}{cccc}
0 & \omega_{Q} & \omega_{Q} & 0 \\
-\omega_{Q} & 0 & 0 & -\omega_{Q} \\
-\omega_{Q} & 0 & 0 & -\omega_{Q} \\
0 & \omega_{Q} & \omega_{Q} & 0
\end{array}\right]
$$

Os elementos das matrizes acima são todos funções da variável espacial $x$. Assim, ao integrar as componentes $\rho_{r s}(x)$ no espaço, ocorrerá um amortecimento dos elementos não diagonais semelhante ao calculado no caso dos gradientes de campo. Da forma das matrizes (2.97), percebe-se que para um campo magnético fortemente não homogêneo, o amortecimento será proporcional ao número de quantum magnético do elemento dado pela matriz $\mathbf{w}_{0}$. Enquanto que para um campo bastante homogêneo, mas com dispersão em $\omega_{Q}$, haverá um amortecimento maior dos elementos em que $\mathbf{w}_{Q}$ é diferente de zero se comparado aos elementos em que $\mathbf{w}_{Q}$ é nulo. 
Aplicando-se um pulso de rotação de $\pi$ radianos e com fase $\phi=0$ sobre o estado $\rho$ do sistema, produz-se o seguinte resultado:

$$
\rho=\left[\begin{array}{llll}
\rho_{11} & \rho_{12} & \rho_{13} & \rho_{14} \\
\rho_{12}^{*} & \rho_{22} & \rho_{23} & \rho_{24} \\
\rho_{13}^{*} & \rho_{23}^{*} & \rho_{33} & \rho_{34} \\
\rho_{14}^{*} & \rho_{24}^{*} & \rho_{34}^{*} & \rho_{44}
\end{array}\right] \Longrightarrow e^{i \pi \mathrm{I}_{x}} \cdot \rho \cdot e^{-i \pi \mathrm{I}_{x}}=\left[\begin{array}{cccc}
\rho_{44} & \rho_{34}^{*} & \rho_{24}^{*} & \rho_{14}^{*} \\
\rho_{34} & \rho_{33} & \rho_{23}^{*} & \rho_{13}^{*} \\
\rho_{24} & \rho_{23} & \rho_{22} & \rho_{12}^{*} \\
\rho_{14} & \rho_{13} & \rho_{12} & \rho_{11}
\end{array}\right]
$$

Comparando-se o efeito do pulso $\pi$ com a evolução descrita pelas matrizes 2.97], percebese que toda evolução devido à interação Zeeman será refocalizada em um tempo simetricamente posterior à aplicação do pulso. No entanto, a evolução quadrupolar permanecerá não refocalizada, contribuindo para a defasagem e conseqüente amortecimento dos elementos em que $\left[\mathbf{w}_{Q}\right]$ é diferente de zero.

Para produzir a refocalização da interação quadrupolar com uma operação unitária seria suficiente realizar as seguintes transformações nos elementos de $\rho$ :

$$
\rho_{12} \leftrightarrow \rho_{34} \quad \text { e } \quad \rho_{13} \leftrightarrow \rho_{24}
$$

Utilizando a indexação lógica da Figura 4, em que os índices de $\rho_{i j}, i, j=1,2,3,4$, correspondem, respectivamente, aos números quânticos $3 / 2,1 / 2,-1 / 2,-3 / 2$, obtém-se a transformação:

$$
|00\rangle\langle 01|\leftrightarrow| 10\rangle\langle 11| \quad \text { e } \quad|00\rangle\langle 10|\leftrightarrow| 01\rangle\langle 11|
$$

Nessa indexação, percebe-se que a transformação da esquerda corresponde à porta lógica $\mathrm{NOT}_{A}$ e a da direita corresponde à $\mathrm{NOT}_{B}$, as quais produzem a negação do primeiro e do segundo q-bit, respectivamente. Logo, caso seja possível construir uma sequiência de pulsos que produza essas portas lógicas obtém-se automaticamente uma seqüência de refocalização para a interação quadrupolar. Note, no entanto, que nesse caso a interação Zeeman permanecerá não refocalizada nos correspondentes elementos.

Caso o objetivo seja a refocalização de todas as interações, a transformação almejada deve $\operatorname{ser} \rho_{i j} \leftrightarrow \rho_{i j}^{*}$. Utilizando-se o mesmo raciocínio empregado para obter a porta de refocalização quadrupolar, conclui-se que a porta $\mathrm{NOT}_{B}$ refocaliza os elementos $\rho_{1,2}$ e $\rho_{3,4}$ enquanto que a porta $\mathrm{NOT}_{A}$ refocaliza os elementos $\rho_{1,3}$ e $\rho_{2,4}$. Deve-se notar que a transformação $\rho_{i j} \leftrightarrow$ $\rho_{i j}^{*}$ refocaliza quaisquer interações estáticas, mesmo aquelas que não foram consideradas na hamiltoniana do sistema devido à baixa intensidade. $\mathrm{O}$ pulso $\pi$ também possui essa propriedade de refocalização universal no caso dos elementos $\rho_{23}$ e $\rho_{14}$, conforme pode ser visto em 2.98. 
Esse efeito também pode ser deduzido observando-se que o pulso $\pi$ com fase 0 corresponde à porta lógica $\mathrm{NOT}^{\otimes 2}$.

Uma possibilidade de geração das portas $\mathrm{NOT}_{A}$ e $\mathrm{NOT}_{B}$ para refocalização é empregando a otimização de pulsos via técnica SMP. No entanto é necessário impor um valor adequado no desvio das interações quadrupolar e Zeeman para que o pulso modulado seja robusto a variações dessas interações. Mesmo sob essas variações a fidelidade da transformação deve ser alta para que a operação de refocalização seja efetiva. Neste trabalho de doutorado foram feitas algumas tentativas preliminares de utilização das portas otimizadas $\mathrm{NOT}_{A}$ e $\mathrm{NOT}_{B}$ para uso em refocalização. No entanto, os resultados experimentais não foram satisfatórios. Acreditamos que provavelmente o problema esteja na baixa eficiência dessas portas. Conforme pode ser observado nos resultados experimentais da seção 4.4, os SMP's mostraram resultados satisfatórios na implementação dos algoritmos quânticos. Todavia, para uso como pulso de refocalização, seria necessário um desempenho significativamente maior. Assim sendo, utilizou-se uma seqüência de spin-eco simples para a refocalização da interação Zeeman nos experimentos de medida de relaxação.

Para descrever o processo de relaxação de todos os elementos da matriz densidade será exposta aqui a dedução da equação mestra para a matriz densidade, a qual envolve a chamada matriz de relaxação de Redfield [30,42]. Essa dedução baseia-se em uma descrição semi-clássica da dinâmica de relaxação pois, embora os estados nucleares sejam tratados quanticamente, a origem dos campos e gradientes temporalmente aleatórios é tratada classicamente.

A hamiltoniana do sistema na ausência de perturbação de rf pode ser escrita como:

$$
\mathrm{H}(t)=\mathrm{H}_{0}+\mathrm{H}_{i n t}+\mathrm{H}^{r}(t)
$$

Em que agora considera-se a hamiltoniana dependente do tempo através da perturbação $\mathrm{H}^{r}(t)$. Essa é uma perturbação aleatória responsável pelo processo de relaxação. Para descrever o seu efeito é conveniente utilizar a representação de interação $\mathrm{H}_{0}+\mathrm{H}_{\text {int }}$ :

$$
\begin{gathered}
\tilde{\rho}=e^{\frac{i}{\hbar}\left(\mathrm{H}_{0}+\mathrm{Hint}\right) t} \rho e^{-\frac{i}{\hbar}\left(\mathrm{H}_{0}+\mathrm{Hint}\right) t} \\
\tilde{\mathrm{H}}^{r}=e^{\frac{i}{\hbar}\left(\mathrm{H}_{0}+\mathrm{H} i n t\right) t} \mathrm{H}^{r} e^{-\frac{i}{\hbar}\left(\mathrm{H}_{0}+\mathrm{H} i n t\right) t} .
\end{gathered}
$$

Resultando na seguinte equação de Liouville-von Neumann:

$$
i \hbar \frac{d \tilde{\rho}}{d t}=\left[\tilde{\mathrm{H}}^{r}, \tilde{\rho}\right]
$$


Utilizando teoria de perturbação dependente do tempo em $2^{a}$ ordem obtém-se, através da integração de (2.103) e substituição do $\tilde{\rho}$ resultante no comutador da mesma expressão 2.103, a seguinte equação:

$$
\hbar^{2} \frac{d \tilde{\rho}}{d t}=-i \hbar\left[\tilde{\mathrm{H}}^{r}(t), \tilde{\rho}(0)\right]-\int_{0}^{t}\left[\tilde{\mathrm{H}}^{r}(t),\left[\tilde{\mathrm{H}}^{r}\left(t^{\prime}\right), \tilde{\rho}\left(t^{\prime}\right)\right]\right] d t^{\prime}
$$

Essa equação se aplica a cada elemento do ensemble de estados distribuídos ao longo da amostra. Portanto, o estado medido corresponderá à média sobre o ensemble. Como a hamiltoniana $\mathrm{H}^{r}$ é aleatória, não há nenhuma correlação com o estado $\tilde{\rho}$, fazendo com que o primeiro termo à direita da igualdade em (2.104) se anule no processo de média. Logo, representando a média sobre o ensemble por \langle\rangle , obtém-se:

$$
\hbar^{2} \frac{d\langle\tilde{\rho}\rangle}{d t}=-\int_{0}^{t}\left\langle\left[\tilde{\mathrm{H}}^{r}(t),\left[\tilde{\mathrm{H}}^{r}\left(t^{\prime}\right), \tilde{\rho}\left(t^{\prime}\right)\right]\right]\right\rangle d t^{\prime} .
$$

Poder-se-ia pensar que o comutador mais interno de (2.105) também se anula no processo de média. No entanto, a média é sobre o comutador mais externo que contém produtos entre a mesma função $\mathrm{H}^{r}$ para tempos muito próximos. Nesses tempos a correlação não é nula, contribuindo para a dinâmica de relaxação. Para evidenciar a correlação entre $\tilde{\mathrm{H}}^{r}(t)$ e $\tilde{\mathrm{H}}^{r}\left(t^{\prime}\right)$ é conveniente fazer a mudança de variável $t^{\prime}=t-\tau$, a qual resulta em:

$$
\hbar^{2} \frac{d\langle\tilde{\rho}\rangle}{d t}=-\int_{0}^{t}\left\langle\left[\tilde{\mathrm{H}}^{r}(t),\left[\tilde{\mathrm{H}}^{r}(t-\tau), \tilde{\rho}(t-\tau)\right]\right]\right\rangle d \tau
$$

Nesse ponto é feita uma restrição sobre a natureza de $\mathrm{H}^{r}(t)$. Assume-se que as correlações que surgem dos produtos entre $\tilde{\mathrm{H}}^{r}(t)$ e $\tilde{\mathrm{H}}^{r}(t-\tau)$ em 2.106 vão a zero em um tempo $\tau$ muito pequeno se comparado ao tempo em que $\tilde{\rho}$ sofre alguma dinâmica. Portanto, nesse intervalo a aproximação $\tilde{\rho}(t-\tau)=\tilde{\rho}(t)$ é válida, fazendo com que o operador $\tilde{\rho}$ no integrando de (2.106) seja independente da variável de integração. Além disso, como a integral vai a zero para intervalos maiores do que esse, o limite de integração pode ser levado a infinito ${ }^{19}$. Com isso, obtém-se:

$$
\hbar^{2} \frac{d\langle\tilde{\rho}\rangle}{d t}=-\int_{0}^{\infty}\left\langle\left[\tilde{\mathrm{H}}^{r}(t),\left[\tilde{\mathrm{H}}^{r}(t-\tau), \tilde{\rho}(t)\right]\right]\right\rangle d \tau .
$$

Pode-se mostrar que os elementos de matriz de 2.107) são dados por:

$$
\hbar^{2} \frac{d\langle\tilde{\rho}\rangle_{i j}}{d t}=-\sum_{k, l}\left[e^{i \omega_{i l} t} J_{i k}^{k l}\langle\tilde{\rho}\rangle_{l j}-e^{i\left(\omega_{i k}+\omega_{l j}\right) t} J_{i k}^{l j}\langle\tilde{\rho}\rangle_{k l}-e^{i\left(\omega_{i k}+\omega_{l j}\right) t} J_{l j}^{i k}\langle\tilde{\rho}\rangle_{k l}+e^{i \omega_{k j} t} J_{l j}^{k l}\langle\tilde{\rho}\rangle_{i k}\right] .
$$

\footnotetext{
${ }^{19}$ Obviamente, esse procedimento é válido para $t$ maior que o tempo de correlação.
} 
Em que $\omega_{i j}=\hbar\left(\left[\mathrm{H}_{0}+\mathrm{H}_{i n t}\right]_{j j}-\left[\mathrm{H}_{0}+\mathrm{H}_{i n t}\right]_{i i}\right)$. As variáveis $J_{i k}^{l j}$ são as chamadas densidades espectrais, definidas como sendo a transformada de Fourier das funções de correlação $g_{i k}^{l j}$ :

$$
J_{i k}^{l j} \equiv \int_{0}^{\infty} g_{i k}^{l j}(t) e^{-i \omega_{l j} \tau} d \tau,
$$

sendo que as funções de correlação são definidas por $g_{i k}^{l j}(t) \equiv\left\langle\mathrm{H}_{i k}^{r}(t) \mathrm{H}_{l j}^{r}(t-\tau)\right\rangle$. As exponenciais em 2.108 surgem devido à transformação de $\tilde{\mathrm{H}}^{r}$ para $\mathrm{H}^{r}$. A fatoração dos elementos $\langle\tilde{\rho}\rangle_{k l}$ em 2.108 é possível devido à condição:

$$
\left\langle\tilde{\mathrm{H}}^{r}(t) \tilde{\mathrm{H}}^{r}(t-\tau) \tilde{\rho}(t)\right\rangle=\left\langle\tilde{\mathrm{H}}^{r}(t) \tilde{\mathrm{H}}^{r}(t-\tau)\right\rangle\langle\tilde{\rho}(t)\rangle
$$

a qual é satisfeita devido ao fato de não haver correlação entre os operadores $\tilde{\mathrm{H}}^{r}(t)$ e $\tilde{\rho}(t)$. É possível deixar em evidência os elementos de matriz de $\tilde{\rho}$ e as exponenciais em 2.108) fazendose uso de deltas de Kronecker, resultando em:

$$
\frac{d}{d t}\langle\tilde{\rho}\rangle_{i j}=\sum_{k, l} e^{i\left(\omega_{i k}-\omega_{j l}\right) t} R_{i j}^{k l}\langle\tilde{\rho}\rangle_{k l}
$$

$R_{k l}^{i j}$ é a matriz de relaxação de Redfield definida por:

$$
R_{i j}^{k l}=J_{i k}^{l j}+J_{l j}^{i k}-\delta_{l j} \sum_{m} J_{i l}^{l m}-\delta_{k i} \sum_{m} J_{k j}^{m k}
$$

A Equação 2.111 pode ser simplificada utilizando-se a aproximação secular. Nessa aproximação, considera-se que podem ser desprezados os termos $k l$ da somatória cujas exponenciais $e^{i\left(\omega_{i k}-\omega_{j l}\right) t}$ oscilam muito rapidamente relativamente à dinâmica do elemento $\rho_{i j}$. Esse é sempre o caso para elementos de diferentes quanta magnéticos, cujas diferenças $\omega_{i k}-\omega_{j l}$ são aproximadamente múltiplas de $\omega_{0}$. Já para elementos diferentes de um mesmo quantum, deve-se verificar se as diferenças de freqüência de acoplamento na hamiltoniana de interação são muito maiores que o inverso do tempo de decaimento desses elementos. Caso não sejam, existirá acoplamento entre os correspondentes elementos.

Analisando o caso do sistema quadrupolar descrito pelas matrizes (2.97), vê-se que para elementos diferentes de um mesmo quantum a diferença $\omega_{i k}-\omega_{j l}$ será ou $\pm \omega_{Q}$ ou $\pm 2 \omega_{Q}$ para as coerências e 0 para as populações. Dos resultados experimentais para a relaxação expostos na seção 4.4 observa-se que, para a amostra usada neste trabalho, o inverso da taxa de decaimento é bem maior que a freqüência $\omega_{Q}$. Portanto, da aproximação secular tem-se para os elementos não diagonais:

$$
\frac{d}{d t}\langle\tilde{\rho}\rangle_{i j}=R_{i j}^{i j}\langle\tilde{\rho}\rangle_{i j} \quad \text { para } \quad i \neq j
$$


Enquanto que para as populações:

$$
\frac{d}{d t}\langle\tilde{\rho}\rangle_{i i}=\sum_{j} R_{i i}^{j j}\langle\tilde{\rho}\rangle_{j j} .
$$

Ou seja, da mesma forma que a Equação (2.79), as coerências possuem um decaimento determinado por uma única exponencial decrescente, com tempo de relaxação $T_{r s}=-1 / R_{r s}^{r s}$. Já as populações possuem um decaimento multi-exponencial. Utilizando-se um único índice para os elementos diagonais pode-se expressar 2.114 na forma matricial: $\frac{d \sigma}{d t}=\mathrm{R} \sigma$. Cuja solução é dada por:

$$
\sigma(t)=e^{\mathrm{R} t} \sigma(0)
$$

Em que $\sigma$ é uma matriz coluna dada por $[\sigma]_{i}=\langle\tilde{\rho}\rangle_{i i}$ e R é a matriz $[\mathrm{R}]_{i j}=R_{i i}^{j j}$. Da definição 2.112 pode-se mostrar que $\mathrm{R}$ é hermitiana, o que permite diagonalizar a exponencial em 2.115. Após esse procedimento, encontra-se a solução para as populações:

$$
\sigma_{i}(t)=\sum_{j} \sigma_{j}(0) \sum_{k} e^{\lambda_{k} t} S_{i k} S_{j k}
$$

em que $S_{i k}$ são os elementos da matriz $\mathrm{S}$ que diagonaliza R: $\Lambda=\mathrm{S}^{-1} \mathrm{RS}$, sendo $[\Lambda]_{k k}=\lambda_{k}$ os auto-valores de R.

Observando a forma da Equação 2.116, percebe-se uma séria inconsistência com relação ao resultado esperado para a matriz densidade de equilíbrio. Isso porque essa equação determina que os valores das populações vão a zero no equilíbrio. A origem dessa inconsistência está no fato de que na dedução dos resultados acima, não foi considerada nenhuma informação sobre o estado térmico da rede. Ao considerar $\mathrm{H}^{r}$ completamente aleatória assumiu-se implicitamente que as taxas de probabilidade de transição entre os diferentes níveis de energia são as mesmas. Esse só seria o caso para a situação de temperatura infinita. Felizmente, pode-se mostrar utilizando-se um tratamento quântico da rede, o qual não será reproduzido aqui e pode ser encontrado na ref. [30], que a equação de relaxação correta é obtida considerando que o operador $\langle\tilde{\rho}\rangle$ nas equações acima corresponde na verdade à diferença com a matriz de equilíbrio térmico:

$$
\langle\tilde{\rho}\rangle=\left\langle\tilde{\rho}^{\prime}\right\rangle-\left\langle\rho_{e q}\right\rangle
$$

em que $\left\langle\tilde{\rho}^{\prime}\right\rangle$ é a matriz densidade correta do sistema.

Conhecendo-se as interações que contribuem para $\mathrm{H}^{r}$ é possível em princípio quantificar a parcela de cada interação para o processo de ralaxação através do cálculo das densidades espectrais 2.109. Devido à possibilidade de decaimento multi-exponencial das populações nessa 
amostra, torna-se interessante tentar verificar sob quais condições a relaxação das mesmas difere de um decaimento mono-exponencial. Embora a equação (2.116 indique que é possível em princípio obter até 4 taxas de decaimento $\lambda_{k}$ distintas para o spin 3/2, será analisado agora o caso simplificado em que assume-se que somente duas taxas de decaimento são significativas para o processo de relaxação. Essa aproximação é empregada pois é mais simples achar uma relação que evidencie a dinâmica multi-exponencial no caso de somente duas taxas de relaxação. Assim, suponha que a dinâmica de uma das populações seja descrita pela seguinte equação:

$$
p(t)=p_{e q}+a e^{-\frac{t}{T_{a}}}+b e^{-\frac{t}{T_{b}}}
$$

O decaimento multi-exponencial de $p$ será mais claramente evidenciado na condição em que $p$ possuir um ponto de máximo. Portanto, calculando a derivada de $p(t)$ e igualando o resultado a zero, obtém-se a seguinte condição para a existência de um máximo para $t>0$ :

$$
b<-a \frac{T_{b}}{T_{a}} \quad \text { para } \quad T_{b}<T_{a} .
$$

Ou seja, quanto mais negativa for a amplitude da componente de relaxação lenta com relação à componente de relaxação rápida, maior será a chance de obter um máximo para a curva de relaxação. Comparando a equação 2.118) com a 2.116, percebe-se que as amplitudes são combinações lineares dos estados iniciais das populações:

$$
\begin{aligned}
& a=\sum_{j} c_{1 j} \sigma_{j}(0) \\
& b=\sum_{j} c_{2 j} \sigma_{j}(0)
\end{aligned}
$$

Dessa forma, é possível em princípio escolher o valor das populações iniciais $\sigma_{j}(0)+\rho_{j}^{e q}$ de forma a satisfazer a condição 2.119 e tentar maximizar o efeito de decaimento multiexponencial. Nesse sentido, os estados pseudo-puros da base computacional representam uma boa escolha de estados iniciais, já que os mesmos formam uma base para os elementos diagonais de $\rho$. Através de combinações lineares específicas das curvas de relaxação desses estados é possível ressaltar a contribuição de um determinado decaimento $\lambda_{k}$ ou então evidenciar o comportamento multi-exponencial através da condição 2.119 por exemplo. A sub-seção 4.4 .4 contém os resultados experimentais obtidos para a relaxação dos estados pseudo-puros. 


\section{3}

\section{TEQ via Rotaç̃̃es Globais do Sistema de Spins}

Conforme discutido na Introdução, o processo de Tomografia de Estado Quântico constituise em uma importante ferramenta para a caracterização dos processos inerentes aos algoritmos quânticos. A primeira proposta de TEQ em RMN foi feita por Chuang et al. [43] e posteriormente aprimorada por Long et al. [44]. Nesses trabalhos, os autores propõem uma técnica de TEQ para sistemas heteronucleares de spins 1/2 acoplados. Conforme discutido na seção 3.4.3. sistemas heteronucleares possibilitam o uso de pulsos não-seletivos em cada um dos núcleos que constituem o sistema. Tais pulsos geram rotações dos spins individuais de forma que, utilizando-se combinações específicas desses pulsos, é possível projetar todas as componentes que expandem a matriz densidade do sistema no operador de medida de RMN.

Conforme visto no capítulo 2, sistemas homonucleares de spin 1/2 e sistemas quadrupolares formados por núcleos isolados também podem ser usados em computação quântica. Para esses casos, pulsos não-seletivos possibilitam somente a rotação global de todos os núcleos de uma mesma espécie. Assim, para discriminar os diferentes estados de tais sistemas, foram propostas diferentes abordagens utilizando-se pulsos seletivos para excitar transições nucleares específicas $[1,31,45,46]$. No entanto, devido à longa duração dos pulsos seletivos, comparativamente aos pulsos não-seletivos utilizados nos sistemas heteronucleares, efeitos de relaxação podem atuar e restringir a utilização do método de tomografia. Essa restrição é tão forte quanto maior for o número de spins envolvidos e maior for o valor desses spins, pois nesses casos são necessárias várias excitações seletivas para determinar todos os elementos de $\rho$ [1]. Com o objetivo de contornar essas dificuldades é que o método de TEQ exposto neste capítulo foi criado. Uma outra proposta interessante de TEQ é a feita por Das et al. [47], em que é empregado o uso da transformada de Fourier bidimensional. Nesse caso, são utilizados somente pulsos não-seletivos para produzir a excitação do sistema. No entanto, a técnica de Fourier bidimensional necessita de tempos de evolução livre antes da aquisição, os quais correspondem a uma das dimensões do espectro bidimensional. Esse método apresenta vantagens para sistemas grandes, para os 
quais torna-se complicado determinar as evoluções específicas necessárias para transferir os elementos da matriz densidade. Uma introdução menos formal ao conteúdo deste capítulo encontra-se no Apêndice A.1

Neste capítulo é demonstrado que a tomografia de núcleos quadrupolares isolados pode ser realizada exclusivamente através de rotações globais do sistema. Embora o método tenha sido desenvolvido particularmente para núcleos quadrupolares isolados, é possível adaptá-lo para sistemas de spins acoplados. Nesse caso é necessário o uso de tempos de evolução livre do sistema, além do uso dos pulsos de rotação não-seletivos. Todos os desenvolvimentos das seções 3.1, 3.2 e 3.3 são gerais e valem para sistemas de spins $1 / 2$ ou quadrupolares sob as interações mais comuns de RMN. Na seção 3.4 o método é restringido a sistemas específicos.

\subsection{Utilização de Pulsos não-seletivos}

Pulsos não-seletivos, também chamados de hard pulses, são pulsos de radiofreqüência relativamente intensos, possuindo a propriedade de promover excitações do sistema de spins em um intervalo de tempo curto se comparado ao tempo que as outras interações presentes na amostra levam para causar alterações significativas no estado do sistema. A descrição da maneira como isso ocorre é o objetivo desta seção.

A hamiltoniana de um sistema formado por $N$ núcleos acoplados pode ser separada nas seguintes contribuições:

$$
\mathrm{H}=\mathrm{H}_{0}+\mathrm{H}_{i n t}+\mathrm{H}_{r f} \text {. }
$$

A hamiltoniana Zeeman e a hamiltoniana de interação são expressas respectivamente por:

$$
\begin{gathered}
\mathrm{H}_{0}=-\hbar \sum_{i=1}^{N} \omega_{0}^{i} \mathrm{I}_{z}^{i} \\
\mathrm{H}_{i n t}=\hbar \sum_{i=1}^{N} \sum_{j=1}^{i} \omega_{i j} \mathrm{H}_{i j}
\end{gathered}
$$

A freqüência $\omega_{0}^{i}=\left(1-\sigma_{i s o}^{i}\right) \gamma_{i} B_{0}$ corresponde à precessão de Larmor juntamente com o efeito de deslocamento químico. Na segunda somatória da Equação 3.3 é adotada a notação em que todas as interações internucleares são englobadas pelas componentes $i>j$, enquanto que as interações quadrupolares são expressas pelas componentes $i=j$. Exemplos de interações internucleares em sólidos e líquidos são mostrados na subseção 2.1.2. Deve-se notar que, de acordo com a Equação (3.3), as contribuições $\mathrm{H}_{i j}$ são adimensionais. 


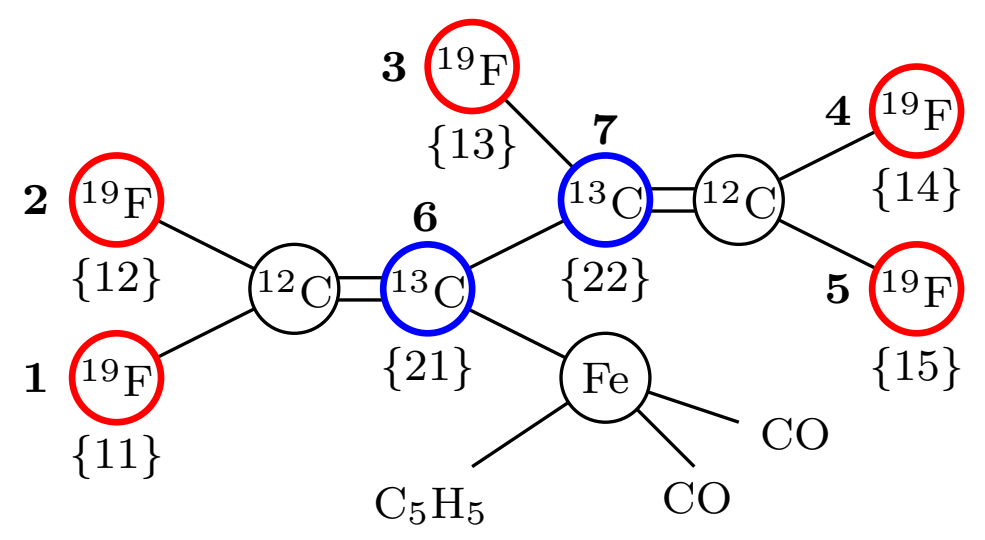

Figura 12: Exemplo da indexação $\{r i\}$ e $k$ (negrito) para as várias espécies nucleares que formam o sistema de spins. Para o caso dessa molécula de ferro perfluorbutadienil o sistema é formado por $N=7$ dipolos nucleares, agrupados em $N_{s}=2$ espécies nucleares com $n_{1}=5$ e $n_{2}=2$ núcleos de cada espécie. Molécula extraída de Ref. [23].

Para obter a hamiltoniana de radiofreqüência , $\mathrm{H}_{r f}$, será utilizado o seguinte pulso:

$$
\mathbf{B}_{\mathbf{1}}(t)= \begin{cases}\sum_{r=1}^{N_{s}} B_{1}^{r}\left[\cos \left(\bar{\omega}_{0}^{r} t+\phi_{r}\right) \hat{\mathbf{x}}+\operatorname{sen}\left(-\bar{\omega}_{0}^{r} t+\phi_{r}\right) \hat{\mathbf{y}}\right] & , \quad 0 \leq t \leq t_{p} . \\ 0 & , \quad \text { resto }\end{cases}
$$

Esse é um pulso retangular de duração $t_{p}$, com componentes harmônicas nas freqüências médias, $\bar{\omega}_{0}^{r}$, de cada espécie nuclear:

$$
\bar{\omega}_{0}^{r} \equiv \frac{1}{n_{r}} \sum_{i=1}^{n_{r}} \omega_{0}^{\{r i\}}
$$

O índice $r$ denota a espécie nuclear e $N_{s}$ corresponde ao número de espécies nucleares distintas que compõem o sistema de spins. O índice com duas variáveis entre chaves, $\{r i\}$, indica que o respectivo parâmetro corresponde ao $i$-ésimo núcleo da $r$-ésima espécie nuclear, sendo que a $r$-ésima espécie contém $n_{r}$ núcleos. O índice $\{r i\}$ pode ser associado univocamente ao índice de uma única variável, $k$, através da relação $k=i+\sum_{j=0}^{r-1} n_{j}$, com $n_{0}=0$. A Figura 12 ilustra esse esquema de indexação.

A hamiltoniana de rf é obtida da energia potencial magnética dos dipolos nucleares com o campo de rf: $\mathrm{H}_{r f}=-\gamma \hbar \mathbf{I} \cdot \mathbf{B}_{\mathbf{1}}$, em que $\mathbf{I}=\sum_{i=1}^{N} \mathbf{I}^{i}$ é a soma dos operadores momento angular de todos os núcleos do sistema. Da Equação (3.4) obtém-se portanto:

$$
\mathrm{H}_{r f}(t)=-\hbar \sum_{r=1}^{N_{s}} \sum_{i=1}^{N} \gamma_{i} B_{1}^{r}\left[\mathrm{I}_{x}^{i} \cos \left(\bar{\omega}_{0}^{r} t+\phi_{r}\right)+\mathrm{I}_{y}^{i} \operatorname{sen}\left(-\bar{\omega}_{0}^{r} t+\phi_{r}\right)\right] .
$$


Neste ponto é conveniente descrever o sistema no referencial multi-giratório, definido como sendo a transformação dos sub-espaços de cada espécie $r$ para o referencial giratório com freqüência $\bar{\omega}_{0}^{r}$. Formalmente, tal mudança é obtida pela transformação unitária:

$$
\mathrm{U}=\exp \left(-i t \sum_{r=1}^{N_{s}} \sum_{i=1}^{n_{r}} \bar{\omega}_{0}^{r} \mathrm{I}_{z}^{r i\}}\right)
$$

Portanto, nesse referencial, a hamiltoniana efetiva $\mathrm{H}^{e f}=\mathrm{UHU}^{\dagger}$, terá as suas componentes transformadas para:

$$
\begin{gathered}
\mathrm{H}_{r f}^{e f}(t)=-\hbar \sum_{r, s=1}^{N_{S}} \sum_{i=1}^{n_{s}} \gamma_{\{s i\}} B_{1}^{r}\left\{\mathrm{I}_{x}^{\{s i\}} \cos \left[\left(\bar{\omega}_{0}^{r}-\bar{\omega}_{0}^{s}\right) t+\phi_{r}\right]+\mathrm{I}_{y}^{\{s i\}} \operatorname{sen}\left[-\left(\bar{\omega}_{0}^{r}-\bar{\omega}_{0}^{s}\right) t+\phi_{r}\right]\right\}, \\
\mathrm{H}_{0}^{e f}=-\hbar \sum_{r=1}^{N_{S}} \sum_{i=1}^{n_{r}}\left(\omega_{0}^{\{r i\}}-\bar{\omega}_{0}^{r}\right) \mathrm{I}_{z}^{\{r i\}}
\end{gathered}
$$

e:

$$
\mathrm{H}_{i n t}^{e f}=\mathrm{H}_{i n t} .
$$

A igualdade na Equação (3.10) decorre do fato de que em $\mathrm{H}_{\text {int }}$ consideram-se somente os termos perturbativos de $1^{a}$ ordem da interação. Assim, as componentes $\mathrm{H}_{\{r i\}\{s j\}}$ da Equação (3.3) comutam com os termos $\bar{\omega}_{0}^{r} \mathbf{I}_{z}^{\{r i\}}+\bar{\omega}_{0}^{s} \mathbf{I}_{z}^{\{s j\}}$ da transformação 3.7, , justificando a igualdade. Devese notar que os termos $\bar{\omega}_{0}^{r} \mathrm{I}_{z}^{r i\}}$ em $\sqrt{3.9}$ são devidos ao surgimento de um campo magnético efetivo na equação de Liouville-von Neumann escrita no novo referencial, pois obviamente $\mathrm{H}_{0}$ também não é modificada pela transformação.

A somatória em $\sqrt{3.8}$ pode ser simplificada, pois as frequiências de oscilação $\left(\bar{\omega}_{0}^{r}-\bar{\omega}_{0}^{s}\right)$ para espécies diferentes, $r \neq s$, são em geral bem maiores que as amplitudes de todas as contribuições nas Equações de (3.8) a (3.10). Portanto, as componentes com essas altas frequiências são desprezadas devido às suas rápidas oscilações, resultando em:

$$
\mathrm{H}_{r f}^{e f}=-\hbar \sum_{r=1}^{N_{S}} \sum_{i=1}^{n r} \gamma_{\{r i\}} B_{1}^{r}\left[\mathrm{I}_{x}^{\{r i\}} \cos \left(\phi_{r}\right)+\mathrm{I}_{y}^{\{r i\}} \operatorname{sen}\left(\phi_{r}\right)\right] .
$$

Dessa forma, com a aproximação (3.11), a hamiltoniana efetiva torna-se independente do tempo, implicando no seguinte propagador para a evolução do estado do sistema:

$$
\mathrm{U}_{R}=e^{-\frac{i}{\hbar} t_{p} \mathrm{H}^{e f}} .
$$

Outra forma de justificar o resultado (3.11), seria observando que as freqüências de transição entre os níveis de energia de $\mathrm{H}^{e f}$ são bem menores que as frequiências $\left(\bar{\omega}_{0}^{r}-\bar{\omega}_{0}^{s}\right), r \neq s$, 
fazendo com que a probabilidade de transição induzida por essas componentes seja desprezível. Essa argumentação seria verdadeira caso o termo $\mathrm{H}_{r f}^{e f}$ fosse uma perturbação de $\mathrm{H}_{0}^{e f}+\mathrm{H}_{i n t}^{e f}$. No entanto, esse não é o caso, pois está sendo considerado que o pulso de rf é não-seletivo e portanto possui a sua intensidade $\omega_{1}^{r}=\gamma_{\{r i\}} B_{1}^{r}$ bem maior que as interações internas $\omega_{i j} \mathrm{e}$ que os desvios por deslocamento químico $\left(\omega_{0}^{\{r i\}}-\bar{\omega}_{0}^{r}\right)$. As relações abaixo resumem a condição necessária para a não-seletividade:

$$
\left|\bar{\omega}_{0}^{r}-\bar{\omega}_{0}^{s}\right| \gg\left|\omega_{1}^{p}\right| \gg\left\{\begin{array}{l}
\left|\omega_{0}^{\{q i\}}-\bar{\omega}_{0}^{q}\right| \\
\left|\omega_{j k}\right|
\end{array} \quad \text { para } r \neq s .\right.
$$

Embora o propagador (3.12) seja simples de ser calculado ele ainda não é satisfatório, pois o interesse em usar pulsos não-seletivos está em fazer com que a evolução do sistema dependa exclusivamente da hamiltoniana do pulso, $\mathrm{H}_{r f}^{e f}$. A forma de garantir essa condição é impondo adicionalmente que:

$$
\left|\omega_{i j}\right| t_{p},\left|\omega_{0}^{\{r i\}}-\bar{\omega}_{0}^{r}\right| t_{p} \ll 1
$$

Dessa forma, com as condições (3.13) e (3.14), o propagador 3.57) se resume a:

$$
\mathrm{U}_{R}=e^{-\frac{i}{\hbar} t_{p} \mathrm{H}^{e f}}=e^{-\frac{i}{\hbar} t_{p} \mathrm{H}_{r f}^{e f}}=\exp \left(i \sum_{r, i} \theta_{r} \mathbf{I}^{\{r i\}} \cdot \hat{\mathbf{u}}_{\phi_{\mathbf{r}}}\right)
$$

Em que $\theta_{r}=\omega_{1}^{r} t_{p}$ e $\hat{\mathbf{u}}_{\phi_{\mathbf{r}}}=\cos \left(\phi_{r}\right) \hat{\mathbf{x}}+\operatorname{sen}\left(\phi_{r}\right) \hat{\mathbf{y}}$. Esse propagador aplica uma rotação no sub-espaço de cada espécie $r$ de um ângulo $\theta_{r}$ em torno da direção $\hat{\mathbf{u}}_{\phi_{\mathbf{r}}}$.

\subsection{Descrição Analítica das Rotações}

Tendo visto que os pulsos não-seletivos possuem a propriedade de promover rotações aproximadamente ideais sobre o estado do sistema de spins, torna-se interessante expandir o operador densidade do sistema em uma base de operadores que apresentem propriedades úteis sob rotações. Uma base que possui essas características é aquela formada pelos chamados Tensores Irredutíveis, os quais foram brevemente tratados no Capítulo 2 na descrição da interação quadrupolar. Nesta seção, tais operadores serão utilizados de uma maneira bastante operacional, sendo que somente as propriedades relevantes para a descrição do método de TEQ serão enunciadas.

Todas as convenções e expressões utilizadas aqui foram obtidas da obra de Varshalovich et al. [48]. Para uma consulta a outros livros importantes sobre Teoria de Momento Angular em Mecânica Quântica vide [34,49-51]. 
Formalmente, os Tensores Irredutíveis $\mathrm{T}_{l m}$ são definidos através das seguintes relações de comutação:

$$
\begin{gathered}
{\left[\mathrm{J}_{ \pm}, \mathrm{T}_{l m}\right]=\mp \frac{1}{2} e^{ \pm i \delta} \sqrt{l(l+1)-m(m \pm 1)} \mathrm{T}_{l, m \pm 1}} \\
{\left[\mathrm{~J}_{0}, \mathrm{~T}_{l m}\right]=m \mathrm{~T}_{l m} .}
\end{gathered}
$$

Os operadores $\mathrm{J}_{ \pm}$e $\mathrm{J}_{0}$ são as componentes esféricas do operador momento angular, as quais se relacionam com as componentes cartesianas através de:

$$
\begin{gathered}
\mathbf{J}_{x}=\frac{1}{\sqrt{2}}\left(\mathrm{~J}_{-}-\mathrm{J}_{+}\right) \\
\mathrm{J}_{y}=\frac{i}{\sqrt{2}}\left(\mathrm{~J}_{-}+\mathrm{J}_{+}\right) \\
\mathrm{J}_{z}=\mathrm{J}_{0} .
\end{gathered}
$$

A imposição das relações de comutação (3.16 garantem que os tensores irredutíveis, sob rotações, se comportem como as auto-funções do operador momento angular. A fase $\delta$ e o sinal a frente do fator $1 / 2$ são arbitrários e apenas definem as fases relativas entre os diferentes $\mathrm{T}_{l m}$.

$\mathrm{O}$ índice $l$ em $\mathrm{T}_{l m}$ define o rank do tensor que pode assumir quaisquer valores inteiros e semi-inteiros positivos. Sendo que para cada rank $l$ são definidas $2 l+1$ componentes $\mathrm{T}_{l m}$ com ordem $m=-l,-l+1, \ldots, l-1, l$.

Para utilizar os tensores irredutíveis como uma base para expandir operadores no espaço de uma partícula de spin $S$ utilizam-se os chamados Operadores de Polarização. Eles são formados pelo conjunto de tensores irredutíveis $\mathrm{T}_{l m}(S)$ de rank $l=0,1, \ldots, 2 S$. Dessa forma, com um conjunto de $\sum_{l=0}^{2 S} 2 l+1=(2 S+1)^{2}$ componentes linearmente independentes, alcança-se o número de graus de liberdade necessários para expandir operadores no espaço de Hilbert da partícula de spin $S$.

Definem-se os operadores de polarização de forma a serem ortonormais e portanto satisfazerem:

$$
\operatorname{Tr}\left\{\mathrm{T}_{l, m}^{\dagger} \cdot \mathrm{T}_{l^{\prime}, m^{\prime}}\right\}=\delta_{l, l^{\prime}} \delta_{m, m^{\prime}}
$$

Além disso os fatores arbitrários em (3.16) são escolhidos de forma a satisfazerem a relação:

$$
\mathrm{T}_{l, m}^{\dagger}=(-1)^{m} \mathrm{~T}_{l,-m}
$$


Com as imposições (3.18) e (3.19) determinam-se completamente os tensores de polarização, os quais possuem a seguinte representação na base de $\mathrm{I}_{z}$ :

$$
\left[\mathrm{T}_{l m}\right]_{\sigma^{\prime}, \sigma}=\sqrt{\frac{2 l+1}{2 S+1}} C_{S \sigma l m}^{S \sigma^{\prime}} .
$$

Em que $C_{S \sigma l m}^{S \sigma^{\prime}}$ são os coeficientes de Clebsh-Gordan e $\sigma^{\prime}, \sigma=S, S-1, \ldots,-S+1,-S$.

Da forma como foram definidos, os operadores de polarização expandem o espaço de um único spin. Para o caso de dois spins acoplados pode-se definir uma nova base de operadores que satisfazem as mesmas relações (3.16). Para isso, utiliza-se o chamado produto irredutível:

$$
\mathrm{T}_{l m}^{L}\left(S_{1}, S_{2}\right)=\sum_{m_{1}, m_{2}} C_{l_{1} m_{1} l_{2} m_{2}}^{l m} \mathrm{~T}_{l_{1} m_{1}}\left(S_{1}\right) \otimes \mathrm{T}_{l_{2} m_{2}}\left(S_{2}\right)
$$

No caso de mais núcleos acoplados, o produto 3.21 pode ser usado recursivamente. Por exemplo, para três spins:

$$
\mathrm{T}_{l^{\prime} m^{\prime}}^{L^{\prime}}\left(S_{1}, S_{2}, S_{3}\right)=\sum_{m, m_{3}} C_{l m l_{3} m_{3}}^{l^{\prime} m^{\prime}} \mathrm{T}_{l, m}^{L}\left(S_{1}, S_{2}\right) \otimes \mathrm{T}_{l_{3}, m_{3}}\left(S_{3}\right)
$$

Com isso, os ranks dos tensores $\mathrm{T}_{l m}^{L}\left(S_{1}, \ldots, S_{N}\right)$ assumem os valores $l=0,1, \ldots, 2 \sum_{i=1}^{N} S_{i}$ com ordem $m=-l,-l+1, \ldots, l-1, l$. O índice $L$ representa os números quânticos adicionais devido ao acoplamento dos spins. Esses números correspondem a:

$$
L=\left\{\begin{array}{l}
l_{1}, l_{2}, \ldots, l_{N} \\
L_{2}, L_{3}, \ldots, L_{N-1}
\end{array} \quad \text { sendo } \quad\left|L_{i-1}-l_{i}\right| \leq L_{i} \leq L_{i-1}+l_{i},\right.
$$

em que $L_{1}=l_{1}$. O rank $l$ é igual a $L_{N}$, obtido ao substituir-se $i=N$ em (3.23). Vale lembrar que a ordem $m$ dos operadores está restrita a $m=\sum_{i} m_{i}$. Da mesma forma que os operadores de polarização, os operadores $\mathrm{T}_{l m}^{L}$ também formam uma base ortonormal, só que agora no espaço produto dos spins $S_{1}, \ldots, S_{N}$. Além disso, eles satisfazem a mesma relação de fase (3.19), a qual independe de $L$.

Como exemplo, a Tabela 1 mostra a relação dos índices $L$ que identificam os operadores $\mathrm{T}_{l m}^{L}$ para o caso de três spins 1/2 acoplados. Para uma descrição mais completa da construção de bases de operadores através de tensores irredutíveis e da escolha dos esquemas de acoplamento entre os spins, vide [52]. 


\begin{tabular}{|c||ccccc|ccccccccc|ccccc|c|}
\hline & 1 & 2 & 3 & 4 & 5 & 6 & 7 & 8 & 9 & 10 & 11 & 12 & 13 & 14 & 15 & 16 & 17 & 18 & 19 & 20 \\
\hline \hline$l_{1}$ & 0 & 0 & 1 & 1 & 1 & 0 & 0 & 0 & 1 & 1 & 1 & 1 & 1 & 1 & 0 & 1 & 1 & 1 & 1 & 1 \\
$l_{2}$ & 0 & 1 & 0 & 1 & 1 & 0 & 1 & 1 & 0 & 0 & 1 & 1 & 1 & 1 & 1 & 0 & 1 & 1 & 1 & 1 \\
$l_{3}$ & 0 & 1 & 1 & 0 & 1 & 1 & 0 & 1 & 0 & 1 & 0 & 1 & 1 & 1 & 1 & 1 & 0 & 1 & 1 & 1 \\
\hline \hline$L_{2}$ & 0 & 1 & 1 & 0 & 1 & 0 & 1 & 1 & 1 & 1 & 1 & 0 & 1 & 2 & 1 & 1 & 2 & 1 & 2 & 2 \\
$l$ & 0 & 0 & 0 & 0 & 0 & 1 & 1 & 1 & 1 & 1 & 1 & 1 & 1 & 1 & 2 & 2 & 2 & 2 & 2 & 3 \\
\hline
\end{tabular}

Tabela 1: Indexação dos tensores irredutíveis que expandem o espaço de operadores para o caso de um sistema formado por três spins $1 / 2$ acoplados. Os coeficientes $l_{1}, l_{2}$ e $l_{3}$ são os ranks dos operadores de polarização dos respectivos spins. $L_{2}$ e $l$ obedecem à regra de soma de momento angular entre $l_{1} l_{2}$ e $L_{2} l_{3}$ respectivamente.

Tendo visto algumas das principais propriedades dos operadores $\mathrm{T}_{l m}^{L}$, eles serão agora utilizados para expandir o operador densidade do sistema de spins:

$$
\rho=\sum_{L, l, m} a_{l m}^{L} \mathrm{~T}_{l m}^{L}
$$

O objetivo do processo de TEQ será portanto determinar os coeficientes $a_{l m}^{L}$. Devido à hermiticidade de $\rho$ e da propriedade (3.19), somente os coeficientes com $m$ positivo precisam ser determinados, pois:

$$
a_{l m}^{L *}=(-1)^{m} a_{l,-m}^{L}
$$

A atuação dos pulsos não-seletivos da seção 3.1 promoverá rotações dos operadores da base $\mathrm{T}_{l m}^{L}$. Para facilitar a descrição serão utilizados pulsos que promovem o mesmo ângulo de nutação $\theta_{r}$ para todas as espécies $r$ do sistema. Esse sempre será o caso para sistemas formados por núcleos quadrupolares isolados ou sistemas exclusivamente homonucleares (que contêm uma única espécie nuclear). Em sistemas heteronucleares, para os quais é possível aplicar ângulos distintos para cada espécie, vide o tratamento da sub-seção 3.4.3. Portanto:

$$
\begin{aligned}
\tilde{\rho} & =\mathrm{U}_{R} \cdot \rho \cdot \mathrm{U}_{R}^{\dagger} \\
& =\sum_{L, l, m} a_{l m}^{L} e^{i \theta I_{\phi}} \cdot \mathrm{T}_{l m}^{L} \cdot e^{-i \theta I_{\phi}},
\end{aligned}
$$

em que se utilizou a notação compacta $\mathrm{I}_{\phi}=\mathbf{I} \cdot \hat{\mathbf{u}}_{\phi}$. Neste ponto será utilizada a propriedade que talvez seja a mais útil dos tensores irredutíveis, qual seja, a transformação dos mesmos sob rotações:

$$
\mathrm{D}(\alpha, \beta, \gamma) \cdot \mathrm{T}_{l m}^{L} \cdot \mathrm{D}^{\dagger}(\alpha, \beta, \gamma)=\sum_{m^{\prime}=-l}^{l} D_{m^{\prime} m}^{l}(\alpha, \beta, \gamma) \mathrm{T}_{l m^{\prime}}^{L} .
$$


O operador $\mathrm{D}(\alpha, \beta, \gamma)$ é o operador rotação escrito em função dos Ângulos de Euler:

$$
\mathrm{D}(\alpha, \beta, \gamma)=e^{-i \alpha \mathrm{I}_{z}} e^{-i \beta \mathrm{I}_{y}} e^{-i \gamma \mathrm{I}_{z}}
$$

As funções $D_{m^{\prime} m}^{l}$ são as Funções de Wigner definidas por:

$$
\left\langle l^{\prime}, m^{\prime}|\mathrm{D}| l, m\right\rangle=\delta_{l^{\prime}, l} D_{m^{\prime} m}^{l},
$$

em que $|l, m\rangle$ são os auto-estados do operador momento angular de número quântico $l$ e projeção $m$. Os Harmônicos Esféricos $Y_{l m}$ também se transformam conforme a Equação 3.27), de acordo com o esperado para as auto-funções do operador momento angular. É possível obter uma expressão mais explícita para as funções de Wigner aplicando a definição (3.29) em (3.28), resultando em:

$$
D_{m^{\prime} m}^{l}(\alpha, \beta, \gamma)=e^{-i m^{\prime} \alpha} d_{m^{\prime} m}^{l}(\beta) e^{-i m \gamma}
$$

A função $d_{m^{\prime} m}^{l}(\beta)=\left\langle l, m^{\prime}\left|e^{-i \beta I_{y}}\right| l, m\right\rangle$, que será chamada de Função de Wigner Reduzida, não possui uma forma única simples, sendo escrita em termos de funções trigonométricas que dependem dos números quânticos $l, m$ e $m^{\prime}$ [48].

A rotação promovida pelos pulsos não-seletivos não é a mais geral possível, pois a mesma está restrita ao plano $x y$ conforme indicado pelo versor $\hat{\mathbf{u}}_{\phi}$. É possível mostrar que os ângulos de Euler, $(\alpha, \beta, \gamma)$, se relacionam com os ângulos do pulso, $(\theta, \phi)$, através das seguintes relações:

$$
\begin{aligned}
& \alpha=-\gamma=\phi-\pi / 2 \\
& \beta=-\theta .
\end{aligned}
$$

Utilizando as Equações 3.31, 3.30) e 3.27) em 3.26) chega-se em:

$$
\tilde{\rho}=\sum_{L, l, m} a_{l, m}^{L} \sum_{m^{\prime}} e^{i\left(m-m^{\prime}\right)\left(\phi-\frac{\pi}{2}\right)} d_{m^{\prime}, m}^{l}(-\theta) \mathrm{T}_{l, m^{\prime}}^{L}
$$

Resta agora calcular a magnetização obtida após a ação do pulso não-seletivo. Em conformidade com a Equação (2.75), encontra-se:

$$
M(t)=\operatorname{Tr}\left\{\mathrm{U}_{f}(t) \cdot \tilde{\rho} \cdot \mathrm{U}_{f}^{\dagger}(t) \cdot \mathrm{I}_{+}\right\} e^{i \alpha},
$$

em que $\mathrm{U}_{f}(t)=e^{-i t\left(\mathrm{H}_{0}^{e f}+\mathrm{H}_{i n t}^{e f}\right) / \hbar}$ é o propagador livre de perturbação de campo de rf, obtido das hamiltonianas 3.9) e 3.10). O ângulo $\alpha$ diz respeito à fase do receptor, não tendo relação com o ângulo de Euler utilizado anteriormente. 
O traço de cada componente $\mathrm{T}_{l m}^{L}$ da expressão da magnetização é facilmente calculado como sendo:

$$
\operatorname{Tr}\left\{\mathrm{T}_{l m}^{L} \cdot \mathrm{U}_{f}^{\dagger} \cdot \mathrm{I}_{+} \cdot \mathrm{U}_{f}\right\}=\sum_{i, j} e^{i \omega_{i j} t}\left[\mathrm{I}_{+}\right]_{i j}\left[\mathrm{~T}_{l m}^{L}\right]_{j i},
$$

em que $\omega_{i j}=\left(E_{i}-E_{j}\right) / \hbar$, sendo $E_{i}$ os auto-estados de $\mathrm{H}_{0}^{e f}+\mathrm{H}_{i n t}^{e f}$. Para o caso do acoplamento escalar fraco em líquidos, para o acoplamento quadrupolar fraco e para o acoplamento dipolar heteronuclear em sólidos, a base computacional, formada pelos auto-estados de $\mathrm{I}_{z}^{j} \mathrm{e}$ $\mathbf{I}^{2 j}, j=1 \ldots N$, coincide com a base de auto-vetores $\left|v_{i}\right\rangle$ de $\mathrm{H}_{0}^{e f}+\mathrm{H}_{i n t}^{e f}$. No entanto, para o acoplamento escalar forte e para o acoplamento dipolar homonuclear, a base computacional não diagonaliza a hamiltoniana efetiva livre, tornando-se necessário calcular os elementos $i j$ em (3.34) na base $\left|v_{i}\right\rangle$.

É possível mostrar que o operador $\mathrm{I}_{+}=\sum_{i=1}^{N} \mathrm{I}_{+}^{i}$ é formado somente por operadores $\mathrm{T}_{l m}^{L} \mathrm{de}$ ordem $m=1$. Além disso, operadores de diferentes ordens não possuem elementos não-nulos nas mesmas posições. Com essas informações e usando a relação (3.19), a Equação (3.34) simplifica-se para:

$$
\operatorname{Tr}\left\{\mathrm{T}_{l m}^{L} \cdot \mathrm{U}_{f}^{\dagger} \cdot \mathrm{I}_{+} \cdot \mathrm{U}_{f}\right\}=\delta_{m,-1} \sum_{i, j} e^{i \omega_{i j} t}\left[\mathrm{I}_{+}\right]_{i j}\left[\mathrm{~T}_{l,-1}^{L}\right]_{j i}
$$

Esse resultado inserido na Equação da magnetização 3.33 fornece o resultado final:

$$
M(t)=\sum_{i, j} f_{i j}(t) S_{i j}
$$

em que:

$$
S_{i j}=\sum_{L, l, m} a_{l m}^{L} e^{i(1+m)\left(\phi-\frac{\pi}{2}\right)+i \alpha} d_{-1, m}^{l}(-\theta)\left[\mathrm{I}_{+}\right]_{i j}\left[\mathrm{~T}_{l,-1}^{L}\right]_{j i}
$$

Conforme discutido na sub-seção 2.1.4, os coeficientes $f_{i j}=e^{i \omega_{i j} t}$ são as oscilações nas correspondentes frequiências de transição e $S_{i j}$ são as respectivas amplitudes espectrais. As funções de Wigner reduzidas, $d_{-1, m}^{l}(-\theta)$, fornecem a dependência angular de cada um dos coeficientes $a_{l m}^{L}$. Para que se tenha uma idéia do comportamento dessas funções com o ângulo de nutação $\theta$, a Figura 13 mostra os gráficos de $d_{-1,2}^{2}(-\theta)$ e $d_{1,2}^{2}(-\theta)$.

Com o objetivo de minimizar o erro na determinação dos coeficientes $a_{l m}^{L}$, devido a erros experimentais no ângulo $\theta$, é interessante aplicar ângulos de nutação, $\theta_{M}$, que maximizem as funções $d_{m^{\prime}, m}^{l}$. No entanto, ângulos grandes implicam em tempos de pulso longos para uma mesma amplitude de rf. Isso faz com que as contribuições de $\mathrm{H}_{0}^{e f}$ e $\mathrm{H}_{\text {int }}^{e f}$ comecem a tornar-se relevantes durante a aplicação do pulso, deixando de satisfazer a condição de nãoseletividade 3.14. Observando os ângulos $\theta_{M}$ para as duas funções da Figura 13 , vê-se que $d_{1,2}^{2}$ 
Comparação entre as Funções de Wigner

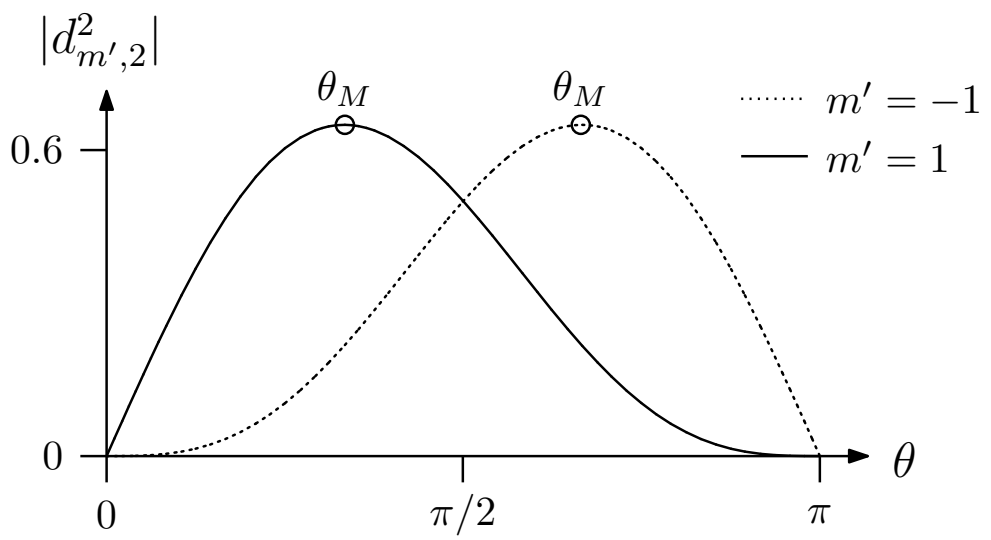

Figura 13: Gráfico das funções de Wigner reduzidas, $d_{m^{\prime}, 2}^{2}(-\theta)$, para $m^{\prime}=1$ e $m^{\prime}=-1$. O valor máximo para o caso $m^{\prime}=1$ é obtido com um ângulo $\theta_{M}$ menor que para o caso $m^{\prime}=-1$.

possui o menor ângulo de maximização. Por inspeção, não só essas funções, mas todas as funções $d_{m^{\prime}, m}^{l}$ possuem $\theta_{M}$ menores nos casos $m^{\prime}=1$ se comparado a $m^{\prime}=-1$. Por esse motivo, será utilizada a propriedade $d_{1, m}^{l}=(-1)^{1-m} d_{-1,-m}^{l}$ [48], para reescrever a Equação 3.37) como:

$$
S_{i j}=\sum_{L, l, m}\left(a_{l m}^{L}\right)^{*} e^{i(1-m)\left(\phi-\frac{\pi}{2}\right)+i \alpha} d_{1, m}^{l}(-\theta)\left[A_{l}^{L}\right]_{i j}
$$

sendo $\left[A_{l}^{L}\right]_{i j}=\left[\mathrm{I}_{+}\right]_{i j}\left[\mathrm{~T}_{l, 1}^{L \dagger}\right]_{j i}$. Identificam-se cinco termos na expressão 3.38. Primeiramente, encontram-se as incógnitas do problema representadas pelos coeficientes $a_{l, m}^{L}$, necessários para reconstruir $\rho$. Em seguida há uma fase que depende da ordem $m$ dos coeficientes, da fase do pulso e da fase do receptor; esses dois últimos podendo ser escolhidos apropriadamente no experimento. Os valores das funções de Wigner para os ângulos de nutação escolhidos são obtidos de obras de referência [48]. Os coeficientes $\left[A_{l}^{L}\right]_{i j}$ acrescentam um peso adicional aos coeficientes $a_{l, m}^{L}$ e podem ser obtidos das expressões 3.20 e 3.21. Por fim, as amplitudes $S_{i j}$ são obtidas das medidas do espectro do sistema utilizando-se os procedimentos expostos na sub-seção 2.1.4 e diferenciam-se entre si apenas pelos coeficientes $\left[A_{l}^{L}\right]_{i j}$.

É possível mostrar que o espectro correspondente à magnetização 3.36 possui no máximo $n_{l}=d \sum_{i=1}^{N} \frac{2 S_{i}}{2 S_{i}+1}$ linhas espectrais, em que $d=\prod_{i=1}^{N}\left(2 S_{i}+1\right)$ é a dimensão do espaço de estados do sistema. Por outro lado, a expressão para as amplitudes espectrais $S_{i j}$ possui contribuições de todos os coeficientes $a_{l, m}^{L}$, os quais formam um conjunto de $n_{a}=d(d+1) / 2-1$ elementos independentes. Em geral $n_{l}<n_{d}$, o que torna necessária a aplicação de rotações adicionais via novos pulso de rf, em que, variando-se os parâmetros experimentais $\alpha, \theta$ e $\phi$, seja possível determinar todos os elementos de $\rho$.

\footnotetext{
${ }^{1}$ Como exemplo, encontra-se para um núcleo quadrupolar isolado de spin 3/2: $n_{l}=3$ e $n_{a}=10$. E para três spins 1/2 acoplados: $n_{l}=12$ e $n_{a}=36$.
} 


\subsection{Seleção de Coerências via Média Temporal}

Com o objetivo de tornar possível a discriminação dos vários elementos que reconstituem a matriz densidade do sistema, será apresentado nesta seção um procedimento de Seleção de Coerências $[11,12,35,53,54]$ que possui a capacidade de reduzir o número de coeficientes presentes em cada linha espectral. Dessa forma, o número de linhas espectrais, $n_{l}$, torna-se compatível com o número reduzido $n_{a}^{\prime}$ de coeficientes selecionados.

O termo coerência é utilizado normalmente para designar quaisquer elementos não-diagonais da matriz densidade. Enquanto que o termo coerências de ordem $m$ é utilizado para designar as coerências que contribuem para os elementos não-nulos do operador $\mathrm{T}_{l, m}^{L}$.

O método de seleção de coerências consiste em um procedimento de médias espectrais que produz espectros dependentes de ordens de coerência específicas da matriz densidade. Através da repetição de vários experimentos, nos quais são variadas as fases dos pulsos de rf e do receptor, realiza-se a chamada Seleção de Coerências via Média Temporal. O termo Temporal refere-se ao fato de que o mesmo experimento, com fases diferentes, é repetido em tempos diferentes, sendo que o espectro final é obtido, posteriormente, a partir da média de todas as repetições. Esse método contrasta-se com a Seleção de Coerências via Média Espacial, em que os gradientes de campo magnético realizam a seleção através da média ao longo do espaço em um único experimento.

Será chamada de $\bar{S}_{i j}$ a amplitude média da linha espectral obtida a partir de $N_{p}$ experimentos, em que para cada experimento variam-se apenas as fases do pulso de rf, $\phi_{n}$, e do receptor, $\alpha_{n}$ :

$$
\bar{S}_{i j}=\frac{1}{N_{p}} \sum_{n=0}^{N_{p}-1} S_{i j}\left(\phi_{n}, \alpha_{n}\right)
$$

O objetivo da média (3.39) é combinar as fases que aparecem na Equação (3.38) para que somente a fase da coerência desejada, $m^{\prime}$, não se anule. Escolhendo-se os seguintes valores para os parâmetros do experimento:

$$
\begin{gathered}
\phi_{n}=2 \pi n / N_{p}+\pi / 2 \\
\alpha_{n}=2 \pi n\left(m^{\prime}-1\right) / N_{p} \\
N_{p} \geq 1+m^{\prime}+2 \sum_{i=1}^{N} S_{i},
\end{gathered}
$$

encontra-se:

$$
\bar{S}_{i j}=\sum_{L, l, m}\left(a_{l m}^{L}\right)^{*} d_{1, m}^{l}(-\theta)\left[A_{l}^{L}\right]_{i j} \sum_{n=0}^{N_{p}-1} e^{i 2 \pi n\left(m^{\prime}-m\right) / N_{p}}
$$


A última somatória de (3.41) produz a seleção de $m^{\prime}$, pois:

$$
\sum_{n=0}^{N_{p}-1} e^{i 2 \pi n\left(m^{\prime}-m\right) / N_{p}}=N_{p} \delta_{m, m^{\prime}},
$$

resultando em:

$$
\bar{S}_{i j}\left(m^{\prime}\right)=\sum_{L, l}\left(a_{l m^{\prime}}^{L}\right)^{*} d_{1, m^{\prime}}^{l}(-\theta)\left[A_{l}^{L}\right]_{i j}
$$

Vale notar que na média (3.39) o ângulo $\theta$ é o mesmo para todos os $N_{p}$ pulsos de rf. Essa restrição é necessária para que seja possível fatorar a somatória nas fases em (3.41).

A Equação (3.43) corresponde ao seguinte sistema linear de equações:

$$
\begin{gathered}
\mathbf{A} \cdot \mathbf{x}=\mathbf{b} \\
{[\mathbf{A}]_{p(i, j), q(L, l)}=\left[A_{l}^{L}\right]_{i j}} \\
{[\mathbf{x}]_{q(L, l)}=\left(a_{l m^{\prime}}^{L}\right)^{*} d_{1, m^{\prime}}^{l}(-\theta)} \\
{[\mathbf{b}]_{p(i, j)}=\bar{S}_{i j}\left(m^{\prime}\right) .}
\end{gathered}
$$

Mesmo com o método de seleção de coerências que resulta no sistema de equações (3.44) não há ainda uma garantia de que o sistema seja solúvel e todos os coeficientes $a_{l m^{\prime}}^{L}$ possam ser determinados. Na verdade, a solubilidade dependerá do sistema nuclear considerado. $\mathrm{Na}$ próxima seção, vários sistemas serão considerados, sendo que para núcleos quadrupolares isolados será dada uma demonstração de que o sistema (3.44) resolve todos os elementos da matriz densidade.

Uma das vantagens da descrição analítica dada nesta seção é o conhecimento a respeito da dependência de cada coeficiente $a_{l m}^{L}$ com o ângulo de flip $\theta$. Portanto, para aprimorar a sensibilidade de uma dada componente $\mathrm{T}_{l m}^{L}$ e reduzir os erros nas rotações dessas componentes, pode-se fazer uma repetição do procedimento de média descrito acima, em que para cada repetição utiliza-se o ângulo de flip $\theta$ que maximiza um determinado rank $l$. Assim, por exemplo, no caso de um núcleo quadrupolar com $S=3 / 2$, para se determinar as coerências de ordem $m=1$ da matriz densidade, repete-se três vezes a série de experimentos que produzem as amplitudes $\bar{S}_{i j}(0)$. Em cada uma das séries utiliza-se o ângulo $\theta$ que maximiza os ranks $l=1, l=2 \mathrm{e}$ $l=3$ respectivamente. 


\subsection{Aplicações}

Os resultados obtidos na seção anterior serão agora aplicados para três casos específicos de interesse prático em RMN e em CQ por RMN. O sistema de equações (3.44) será discutido para cada caso, juntamente com algumas simulações do processo de TEQ. Os resultados experimentais são apresentados no Capítulo 4.

\subsubsection{Sistemas de Spins Quadrupolares Isolados}

Para o sistema de núcleos quadrupolares isolados é possível simplificar as equações (3.43) e (3.44). Como foi discutido anteriormente, nesse caso a base de operadores será constituída diretamente pelos operadores de polarização (3.20). Assim, todas as expressões da seção anterior serão utilizadas aqui sem o índice super-escrito $L$ responsável pelos graus de liberdade devido ao acoplamento entre spins.

$\mathrm{Na}$ base de auto-vetores de $\mathrm{I}_{z}$ os operadores de polarização $\mathrm{T}_{l m}(S)$ possuem uma propriedade interessante com relação à ordem de coerência $m$, qual seja, a de que somente os elementos $\left[\mathrm{T}_{l m}\right]_{i, i+m}$ são não nulos. Como exemplo, o Apêndice A.8 contém a forma dos operadores $\mathrm{T}_{l m}(S)$ para $S=3 / 2$. Portanto, os elementos $\left[A_{l}\right]_{i j}$ em 3.43 tornam-se iguais a:

$$
\left[A_{l}\right]_{i j}=\delta_{i+1, j}\left[\mathrm{I}_{+}\right]_{i j}\left[\mathrm{~T}_{l, 1}^{\dagger}\right]_{j i}
$$

resultando nas seguintes amplitudes espectrais:

$$
\bar{S}_{i, i+1}\left(m^{\prime}\right)=\sum_{l}\left(a_{l m^{\prime}}\right)^{*} d_{1, m^{\prime}}^{l}(-\theta)\left[A_{l}\right]_{i, i+1}
$$

Neste ponto é necessário discutir a questão da sensibilidade do experimento de RMN à todos os elementos da matriz densidade. Conforme discutido no Capítulo 2, a parcela da matriz densidade proporcional à identidade não pode ser medida pela técnica tradicional de RMN, sendo possível apenas a medida do chamado operador densidade de desvio: $\Delta \rho=\rho-\alpha \mathbf{1}$. Na base de operadores de polarização, o termo identidade é proporcional ao operador $\mathrm{T}_{00}$, fazendo com que o operador densidade de desvio não contenha essa contribuição:

$$
\Delta \rho=\sum_{l=1}^{2 S} \sum_{m=-l}^{l} a_{l m} \mathrm{~T}_{l m} .
$$


Além disso, o operador $\Delta \rho$ dado por (3.47) possui traço nulo, pois:

$$
\begin{aligned}
\operatorname{Tr}\{\Delta \rho\} \propto \operatorname{Tr}\left\{\mathrm{T}_{00} \cdot \Delta \rho\right\} & =\sum_{l=1}^{2 S} \sum_{m=-l}^{l} a_{l m} \operatorname{Tr}\left\{\mathrm{T}_{00} \cdot \mathrm{T}_{l m}\right\} \\
& =\sum_{l=1}^{2 S} \sum_{m=-l}^{l} a_{l m} \delta_{0, l} \delta_{0, m} \\
& =0,
\end{aligned}
$$

em que na passagem para a segunda linha foi usada a propriedade 3.18). Vale notar que a impossibilidade de determinar o coeficiente $a_{00}$ está implícita na Equação (3.45), pois os operadores $\mathrm{T}_{l m}$ só estão definidos para $l \geq|m|$. Com base nessa discussão, a soma em $l$ na Equação 3.46) é restrita a:

$$
\begin{gathered}
\bar{S}_{i, i+1}\left(m^{\prime}\right)=\sum_{\substack{l=l^{\prime} \\
l^{\prime}}}^{2 S}\left(a_{l m^{\prime}}\right)^{*} d_{1, m^{\prime}}^{l}(-\theta)\left[A_{l}\right]_{i, i+1} \\
\max \left(1, m^{\prime}\right)
\end{gathered}
$$

O sistema linear correspondente torna-se:

$$
\left.\begin{array}{c}
\mathbf{A} \cdot \mathbf{x}=\mathbf{b} \\
{[\mathbf{A}]_{i, l-l^{\prime}+1}=\left[A_{l}\right]_{i, i+1}} \\
{[\mathbf{x}]_{l-l^{\prime}+1}=\left(a_{l m^{\prime}}\right)^{*} d_{1, m^{\prime}}^{l}(-\theta)} \\
{[\mathbf{b}]_{i}=\bar{S}_{i, i+1}\left(m^{\prime}\right)}
\end{array}\right\} \quad \begin{gathered}
i=1,2, \ldots, 2 S \\
l=l^{\prime}, l^{\prime}+1, \ldots, 2 S
\end{gathered}
$$

Vê-se portanto, que o sistema (3.49) é formado por $2 S$ equações e $2 S-l^{\prime}+1$ incógnitas. O caso em que $m^{\prime}=0$ e $m^{\prime}=1$, implica em $l^{\prime}=1$, o que torna o sistema linear determinado, formado pela matriz de coeficientes $\mathbf{A}_{2 S \times 2 S}$. Para $m^{\prime}>1$ a matriz A sempre será retangular, com o número de equações maior que o número de incógnitas. Um sistema desse tipo pode ser resolvido utilizando-se o Método de Mínimos Quadrados para sistemas lineares de equações. Essa redundância na informação das coerências de ordem maior que 1, pode parecer implicar em medidas relativamente mais precisas para essas coerências. No entanto, como mostrado no Apêndice A.8. os máximos das funções de Wigner $d_{1, m^{\prime}}^{l}$ diminuem com o aumento de $m^{\prime}$, o que significa uma concomitante perda de sensibilidade.

Para garantir que o sistema (3.49) é de fato sempre determinado, falta ainda verificar que o mesmo possui $2 S-l^{\prime}+1$ equações linearmente independentes. Uma demonstração mais direta dessa proposição pode ser obtida ao observar-se que cada coluna $l-l^{\prime}+1$ de $\mathbf{A}$ é proporcional ao vetor formado pelos elementos não nulos de $\mathrm{T}_{l, 1}$. Portanto, como os operadores de polarização são linearmente independentes entre si (pois formam uma base), assim são as colunas de $\mathbf{A}$, 


\begin{tabular}{|c||c||cccccccc|}
\hline & $n$ & 1 & 2 & 3 & 4 & 5 & 6 & 7 & 8 \\
\hline \hline \multirow{2}{*}{$S=3 / 2$} & $\alpha$ & 0 & $3 \pi / 2$ & $\pi$ & $\pi / 2$ & & & & \\
& $\phi$ & $\pi / 2$ & $\pi$ & $3 \pi / 2$ & 0 & & & & \\
\hline \hline \multirow{2}{*}{$S=7 / 2$} & $\alpha$ & 0 & $7 \pi / 4$ & $3 \pi / 2$ & $5 \pi / 4$ & $\pi$ & $3 \pi / 4$ & $\pi / 2$ & $\pi / 4$ \\
& $\phi$ & $\pi / 2$ & $3 \pi / 4$ & $\pi$ & $5 \pi / 4$ & $3 \pi / 2$ & $7 \pi / 4$ & 0 & $\pi / 4$ \\
\hline
\end{tabular}

Tabela 2: Fases dos pulsos de rf, $\phi$, e do receptor, $\alpha$, necessárias para tomografar os elementos diagonais, $m^{\prime}=0$, da matriz densidade dos núcleos de spin $3 / 2$ e $7 / 2$. Para o caso do spin $3 / 2$ as fases coincidem com a ciclagem CYCLOPS.

garantindo a solubilidade do sistema.

Uma vez apresentado o método de TEQ para núcleos quadrupolares, surge a oportunidade de fazer-se uma comparação com o método de TEQ desenvolvido por Bonk et al. [3,7]. Nesse trabalho, os autores apresentaram um método de tomografia para núcleos de spin 3/2, em que os elementos diagonais (populações) da matriz densidade são obtidos através da ciclagem de pulsos de rf conhecida como CYCLOPS. Nessa ciclagem são utilizados 4 pulsos com as fases mostradas na Tabela 2. Nota-se, que ao utilizar-se as Equações (3.40 para o caso $S=3 / 2$ obtém-se as mesmas fases da Tabela 2 para os elementos diagonais, $m^{\prime}=0$. Portanto, podese dizer que o método proposto nesta tese representa uma generalização do trabalho anterior. Como ilustração, também é apresentada na Tabela 2 a ciclagem de fases necessária para tomografar os elementos diagonais no caso $S=7 / 2$ utilizando-se os parâmetros (3.40).

Mais uma vez é possível apreciar a vantagem de se utilizar a descrição analítica apresentada neste capítulo. Pois, ao observar-se o comportamento das funções de Wigner para as coerências de ordem 0, mostradas na Figura 57 do Apêndice A.8, percebe-se que um ângulo de nutação de $\pi / 2$ nem sempre é a melhor opção para determinar-se os elementos diagonais de $\rho$. Conforme mostra a figura, esse ângulo é o ideal para determinar-se a componente $\mathrm{T}_{1,0}$, que por sinal é proporcional ao estado de equilíbrio $\mathrm{I}_{z}$, justificando a escolha desse ângulo em experimentos que aplicam pulsos sobre o estado de equilíbrio. No entanto, vê-se que a componente $\mathrm{T}_{2,0}$ torna-se totalmente indeterminada em $\theta=\pi / 2$, impossibilitando a leitura de estados que sejam fortemente dependentes dessa componente.

Como um teste preliminar para o método de TEQ em núcleos quadrupolares é apresentada agora uma simulação do processo de tomografia.

Como ponto de partida foi utilizado um operador densidade de desvio referente ao seguinte estado superposto de 3 q-bits:

$$
|\Psi\rangle=\frac{1}{2 \sqrt{2}} \bigotimes_{i=1}^{3}\left(|0\rangle_{i}+|1\rangle_{i}\right)
$$


a)

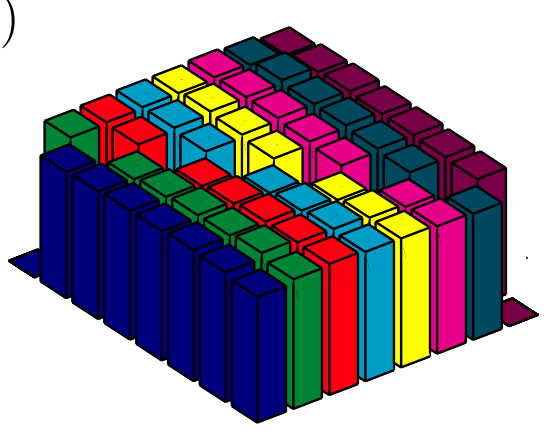

b)

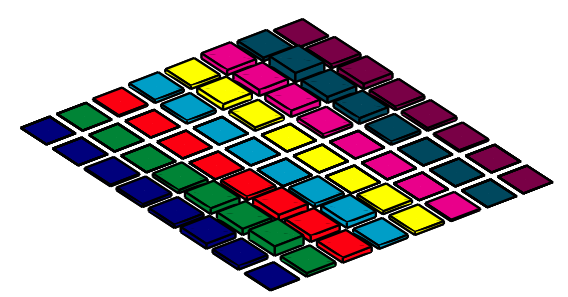

Figura 14: Simulação do método de tomografia para um sistema de spin $7 / 2$. (a) Tomografia do estado correspondente a superposição $|\Psi\rangle=\frac{1}{2 \sqrt{2}} \bigotimes_{i=1}^{3}\left(|0\rangle_{i}+|1\rangle_{i}\right)$. (b) Desvio absoluto com relação à matriz de desvio dada pela Equação (3.51). Todos os pulsos de radiofrequiência foram aplicados com um erro sistemático de $5 \%$ no ângulo de nutação. As cores em todos os gráficos de barras servem apenas para facilitar a distinção visual dos elementos da matriz densidade. Estão mostradas somente as componentes reais de cada elemento.

Que corresponde à seguinte matriz de desvio:

$$
\Delta \rho=|\Psi\rangle\langle\Psi|-\frac{1}{8} \mathbb{1}=\frac{1}{8}\left[\begin{array}{llllllll}
0 & 1 & 1 & 1 & 1 & 1 & 1 & 1 \\
1 & 0 & 1 & 1 & 1 & 1 & 1 & 1 \\
1 & 1 & 0 & 1 & 1 & 1 & 1 & 1 \\
1 & 1 & 1 & 0 & 1 & 1 & 1 & 1 \\
1 & 1 & 1 & 1 & 0 & 1 & 1 & 1 \\
1 & 1 & 1 & 1 & 1 & 0 & 1 & 1 \\
1 & 1 & 1 & 1 & 1 & 1 & 0 & 1 \\
1 & 1 & 1 & 1 & 1 & 1 & 1 & 0
\end{array}\right] .
$$

O sistema físico sobre o qual o método de TEQ foi aplicado corresponde a um sistema quadrupolar de spin $S=7 / 2$. Nesta simulação, todos os pulsos de radiofreqüência foram assumidos como sendo pulsos que produzem as rotações ideais dadas pelo propagador (3.15). Ou seja, não foi considerado o efeito da hamiltoniana de interação quadrupolar $\mathrm{H}_{\text {int }}$ ao longo da aplicação dos pulsos. O único erro considerado nessa simulação foi nos ângulos de nutação $\theta$, sobre os quais considerou-se um erro sistemático de 5\% para todos os pulsos.

Os ângulos de nutação utilizados foram aqueles que maximizam as respectivas funções de Wigner, $d_{1, m^{\prime}}^{l}$. Dessa forma, a seleção de cada coerência $m^{\prime}$ foi repetida $2 S-l^{\prime}+1$ vezes, de forma a maximizar a sensibilidade de cada rank $l$. Portanto, a matriz de coeficientes A obtida para cada sistema linear de cada coerência $m^{\prime}$ possui dimensão $\left[2 S\left(2 S-l^{\prime}+1\right)\right] \times\left[2 S-l^{\prime}+1\right]$. O resultado da tomografia encontra-se na Figura 14(a). A Figura 14(b) mostra o desvio com relação ao estado original (3.51). O máximo desvio obtido foi de $7 \%$. 

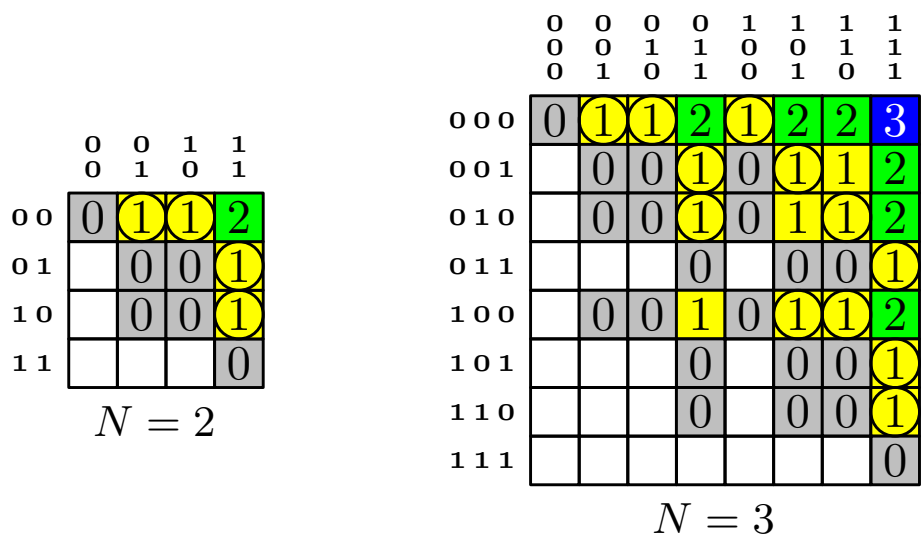

Figura 15: Dependência da matriz densidade com a ordem de coerência $m$ dos operadores $\mathrm{T}_{l m}^{L}$ para os casos de $N=2$ e $N=3$ spins $1 / 2$ acoplados. Os elementos de $\rho$ estão marcados com a ordem de coerência que contribui na posição desses elementos. Os elementos marcados com círculos são aqueles que recebem contribuição do operador $\mathrm{I}_{+}$. Somente as coerências não negativas estão indicadas e a representação utilizada é na base de $\mathrm{I}_{z}^{i}$ e $\mathbf{I}^{{ }^{i}}$.

\subsubsection{Sistemas de Spins Homonucleares Acoplados}

Quando o sistema de interesse é composto somente por núcleos da mesma espécie, o efeito da aplicação de pulsos não-seletivos é ainda descrito pelas Equações (3.43) e (3.44). No entanto, não é possível achar uma regra simples para a estrutura dos operadores $\mathrm{T}_{l m}^{L}$, de tal forma que os elementos $\left[A_{l}^{L}\right]_{i j}$ sejam simplificados como foi feito para os elementos $\left[A_{l}\right]_{i j}$ em 3.45 . Para se ter uma idéia da forma desses operadores, a Figura 15 mostra a matriz densidade, na base de $\mathrm{I}_{z}^{i}$ e $\mathbf{I}^{{ }^{i}}$, para os casos de $N=2$ e $N=3$ spins 1/2 acoplados. Em cada caso, as matrizes densidade estão marcadas com o valor da ordem $m \geq 0$ do operador $\mathrm{T}_{l m}^{L}$ que contribui para cada elemento. Os elementos que possuem contribuição do operador $\mathrm{I}_{+}$estão marcados com círculos.

Percebe-se uma importante distinção ao comparar os casos de 2 e 3 spins. Para 2 spins acoplados, todos os operadores $\mathrm{T}_{l m}^{L}$ de ordem 1 possuem todos os elementos em comum com o operador $\mathrm{I}_{+}$. No entanto, para três spins, nota-se que os elementos de $\mathrm{T}_{l, 1}^{L}$ contidos na diagonal secundária de $\rho$ não são abrangidos por $\mathrm{I}_{+}$. Devido a essa característica, não é possível determinar todos os elementos de ordem $m^{\prime}=1$ usando o procedimento de seleção de coerências dado pela Equação (3.40) e através da aplicação de um único ângulo de nutação $\theta$. Esse problema ocorre pois as amplitudes espectrais $S_{i j}$ dependem dos elementos $\left[A_{l}^{L}\right]_{i j}=\left[\mathrm{I}_{+}\right]_{i j}\left[\mathrm{~T}_{l, 1}^{L \dagger}\right]_{j i}$, que por sua vez precisam ter elementos $\left[\mathrm{I}_{+}\right]_{i j}$ e $\left[\mathrm{T}_{l, 1}^{L}\right]_{i j}$ não nulos em comum para produzir as respectivas linhas $S_{i j}$. No caso de um spin $S$ quadrupolar isolado, discutido na sub-seção anterior, o operador $\mathrm{I}_{+}$é proporcional ao operador de polarização $\mathrm{T}_{1,1}$, que por sua vez possui todos os elementos comuns aos operadores $\mathrm{T}_{l, 1}$.

Resumindo, para sistemas de spins acoplados, em geral, existem mais coerências de or- 
dem 1 do que aquelas abrangidas pelo operador $\mathrm{I}_{+}$. Conseqüentemente, o espectro obtido em um único experimento não possui um número de graus de liberdade grande o suficiente para determinar todos os elementos de uma dada ordem de coerência.

Para resolver este problema pode-se utilizar o procedimento descrito anteriormente, de repetir para vários ângulos de nutação $\theta$ o experimento de seleção da coerência $m^{\prime}$ e assim aumentar o número de equações do sistema linear (3.44). Diferentemente do caso de núcleos quadrupolares isolados, não será dada aqui uma demonstração geral que garanta quais são as condições para que o sistema linear (3.44) seja determinado. Cada sistema homonuclear deve ser analisado particularmente para que se encontre o número de pulsos necessários e os ângulos de nutação ideais de tal forma que a tomografia seja bem sucedida.

Existe uma segunda dificuldade relacionada à aplicação do método de TEQ proposto neste capítulo a sistemas de núcleos acoplados. Voltando à Tabela 1 na página 64, nota-se que existem mais componentes de rank $l=0$ do que o termo proporcional à identidade, $\mathrm{T}_{0,0}^{0}$. Essa característica acarreta um problema sério ao método de tomografia com pulsos exclusivamente nãoseletivos. Isso porque, primeiramente, essas componentes, por não serem proporcionais à identidade, evoluem sob transformações unitárias. Em segundo lugar, operadores de rank 0 não são observados através da aplicação de pulsos não-seletivos. Isso pode ser visto na Equação (3.27) que diz que o rank $l$ do tensor não é alterado por operações de rotação. Assim, como o rank deve satisfazer $l \geq|m|$, não é possível levar as componentes $a_{0,0}^{L}$ para os elementos de ordem 1 que contribuem para as linhas espectrais.

Para resolver esse segundo problema, uma solução é permitir que o sistema evolua sob a hamiltoniana não perturbada $\mathrm{H}_{0}^{e f}+\mathrm{H}_{i n t}^{e f}$, a qual pode produzir a evolução dos tensores de rank 0 para um rank mais alto. Como foi discutido na seção 3.2, a matriz densidade do sistema é descrita na base $\left|v_{i}\right\rangle$ em que a hamiltoniana não perturbada é diagonal. Portanto, sob essa hamiltoniana, não há a mistura dos elementos de $\rho$. Cada elemento ganha apenas uma fase correspondente, o que, conforme discussão da Equação 3.35, não altera a ordem das coerências. Dessa forma, pode-se aplicar o experimento de seleção de coerência de ordem 0 após um determinado tempo de evolução livre, para se determinar todos os ranks dessa ordem.

\section{Sistema de equações com tempo de evolução livre}

Dado o propagador de evolução livre $\mathrm{U}_{f}(t)=e^{-i t\left(\mathrm{H}_{0}^{e f}+\mathrm{H}_{\text {int }}^{e f}\right) / \hbar}$, a transformação dos operadores da base pode ser representada por:

$$
\tilde{\mathrm{T}}_{l m}^{L} \equiv \mathrm{U}_{f} \cdot \mathrm{T}_{l m}^{L} \cdot \mathrm{U}_{f}^{\dagger}=\sum_{L^{\prime}, l^{\prime}} b_{l l^{\prime}}^{L L^{\prime}} \mathrm{T}_{l^{\prime} m}^{L^{\prime}} .
$$


Deve-se notar que não ocorre alteração da ordem de coerência dos operadores. Aplicandose todo o desenvolvimento da Seção 3.2 sobre o operador densidade evoluído:

$$
\rho=\sum_{L, l, m} a_{l, m}^{L} \tilde{\mathrm{T}}_{l m}^{L}
$$

encontra-se facilmente o seguinte resultado para as linhas espectrais:

$$
S_{i j}=\sum_{L, l, m}\left(a_{l m}^{L}\right)^{*} e^{i(1-m)\left(\phi-\frac{\pi}{2}\right)+i \alpha} \sum_{L^{\prime}, l^{\prime}} d_{1, m}^{l^{\prime}}(-\theta) b_{l l^{\prime}}^{L L^{\prime}}\left[A_{l^{\prime}}^{L^{\prime}}\right]_{i j}
$$

Ao aplicar o procedimento de seleção de coerências para a ordem 0 obtém-se o seguinte sistema de equações:

$$
\begin{gathered}
\mathbf{A} \cdot \mathbf{x}=\mathbf{b} \\
{[\mathbf{A}]_{p(i, j), q(L, l)}=\sum_{L^{\prime}, l^{\prime}} d_{1,0}^{l^{\prime}}(-\theta) b_{l l^{\prime}}^{L L^{\prime}}\left[A_{l^{\prime}}^{L^{\prime}}\right]_{i j}} \\
{[\mathbf{x}]_{q(L, l)}=\left(a_{l, 0}^{L}\right)^{*}} \\
{[\mathbf{b}]_{p(i, j)}=\bar{S}_{i j}(0)}
\end{gathered}
$$

A idéia, portanto, é encontrar os tempos de evolução livre que maximizam os coeficientes $b_{0, l}^{L L^{\prime}}$ e assim obter informação sobre as componentes $a_{0,0}^{L}$.

\section{Simulação da TEQ em um sistema homonuclear}

Para ilustrar o procedimento de TEQ em um sistema homonuclear, será mostrada a seguir a simulação da tomografia do estado superposto (3.51) em um sistema de 3 spins 1/2 homonucleares acoplados. Foi considerada uma amostra líquida fictícia em que a hamiltoniana de interação do sistema é descrita pelo acoplamento escalar fraco, resultando na seguinte hamiltoniana efetiva não perturbada:

$$
\mathrm{H}^{e f}=-\hbar \sum_{i=1}^{3}\left(\omega_{0}^{i}-\bar{\omega}_{0}\right) \mathrm{I}_{z}^{i}+2 \pi \hbar \sum_{i=1}^{3} \sum_{j<i} J_{i j} \mathrm{I}_{z}^{i} \mathrm{I}_{z}^{j}
$$

Os valores dos acoplamentos $J_{i j}$ são dados pelos termos não diagonais na Tabela 3 , sendo que os deslocamentos químicos $\omega_{0}^{i}-\bar{\omega}_{0}$ estão contidos na diagonal $i=j$. Como esse é um sistema de 3 spins $1 / 2$, existem $3 \times(2 \times 1 / 2)=3$ ordens de coerência a serem tomografadas. Os pulsos utilizados no processo de tomografia seguiram a forma dada pela Equação (3.40, sendo que a Tabela 4 contém explicitamente as fases utilizadas. O experimento de seleção de cada ordem de coerência foi repetido para os ângulos de nutação da Tabela 5, os quais maximi- 


\begin{tabular}{|c|ccc|}
\hline$i \backslash j$ & 1 & 2 & 3 \\
\hline 1 & -170 & & \\
2 & 5 & 50 & \\
3 & 8 & 3 & 120 \\
\hline
\end{tabular}

Tabela 3: Acoplamentos escalares $J_{i j}$ e deslocamentos químicos $\omega_{0}^{i}-\bar{\omega}_{0}$ (diagonal) para o sistema homonuclear simulado de 3 spins 1/2. Todos os valores estão em hertz. Sobre esse sistema foi aplicado o método de TEQ para núcleos acoplados.

\begin{tabular}{|c||cccccccc|}
\hline \multicolumn{1}{l||}{} & \multicolumn{2}{c}{$m=0$} & $m=1$ & \multicolumn{2}{c}{$m=2$} & \multicolumn{2}{c}{$m=3$} \\
$n$ & $\phi$ & $\alpha$ & $\phi$ & $\alpha$ & $\phi$ & $\alpha$ & $\phi$ & $\alpha$ \\
\hline \hline 0 & $\pi / 2$ & 0 & $\pi / 2$ & 0 & $\pi / 2$ & 0 & $\pi / 2$ & 0 \\
1 & $\pi$ & $3 \pi / 2$ & $9 \pi / 10$ & 0 & $5 \pi / 6$ & $\pi / 3$ & $11 \pi / 4$ & $2 \pi / 7$ \\
2 & $3 \pi / 2$ & $\pi$ & $13 \pi / 10$ & 0 & $7 \pi / 10$ & $2 \pi / 3$ & $15 \pi / 4$ & $4 \pi / 7$ \\
3 & 0 & $\pi / 2$ & $17 \pi / 10$ & 0 & $3 \pi / 2$ & $\pi$ & $19 \pi / 4$ & $6 \pi / 7$ \\
4 & & & $21 \pi / 10$ & 0 & $11 \pi / 6$ & $4 \pi / 3$ & $23 \pi / 4$ & $8 \pi / 7$ \\
5 & & & & & $13 \pi / 6$ & $5 \pi / 3$ & $27 \pi / 4$ & $10 \pi / 7$ \\
6 & & & & & & & $31 \pi / 4$ & $12 \pi / 7$ \\
\hline
\end{tabular}

Tabela 4: Fases $\phi$ dos pulsos de rf e fases $\alpha$ do recptor para os experimentos de seleção de coerência no caso do spin $3 / 2$, em radianos. Cada coluna $m$ corresponde a um experimento para a seleção da $m$-ésima coerência. Os ângulos de nutação são usados de acordo com a tabela 5 .

\begin{tabular}{|c|c|c|c|c|}
\hline$l$ & $m=0$ & $m=1$ & $m=2$ & $m=3$ \\
\hline 1 & $\pi / 2$ & 0 & & \\
\hline 2 & $\pi / 4$ & 0 & $\pi / 3$ & \\
\hline 3 & 0.54 & $0 \quad 1.16$ & 0.60 & 1.23 \\
\hline
\end{tabular}

Tabela 5: Ângulos de nutação $\theta$, em radianos, que maximizam a sensibilidade espectral de ranks $l$ específicos da matriz densidade do sistema.

zam a contribuição de cada rank $l$. Para o experimento de seleção das coerências de ordem 1 , foi utilizado o ângulo adicional de 1,16 radianos, necessário para se obter o número mínimo de equações linearmente independentes que determinam todas as coerências de $1^{a}$ ordem, conforme discutido acima. Foi escolhido esse segundo ângulo, pois ele é o menor ângulo, depois de zero, que maximiza um dos ranks de ordem 1 (vide Figura 57). Para essa simulação também foram usados pulsos não-seletivos ideais, sendo que o único erro considerado foi novamente um erro sistemático de $5 \%$ no ângulo de nutação dos pulsos.

Observando a Tabela 1 na página 64, nota-se que existem 4 operadores de rank 0 além do operador proporcional à identidade. Para determinar quais são os tempos, sob evolução livre, necessários para a máxima transferência para um $\operatorname{rank} l>0$ é útil calcular a projeção sobre $\mathrm{T}_{l, 0}^{L}$ :

$$
P_{L L^{\prime}}^{l}(t) \equiv \operatorname{Tr}\left\{\mathrm{T}_{l, 0}^{L \dagger} \cdot \mathrm{U}_{f}(t) \cdot \mathrm{T}_{0,0}^{L^{\prime}} \cdot \mathrm{U}_{f}^{\dagger}(t)\right\}=b_{0, l}^{L^{\prime} L}
$$


Transferência das componentes de rank 0 para o rank 2

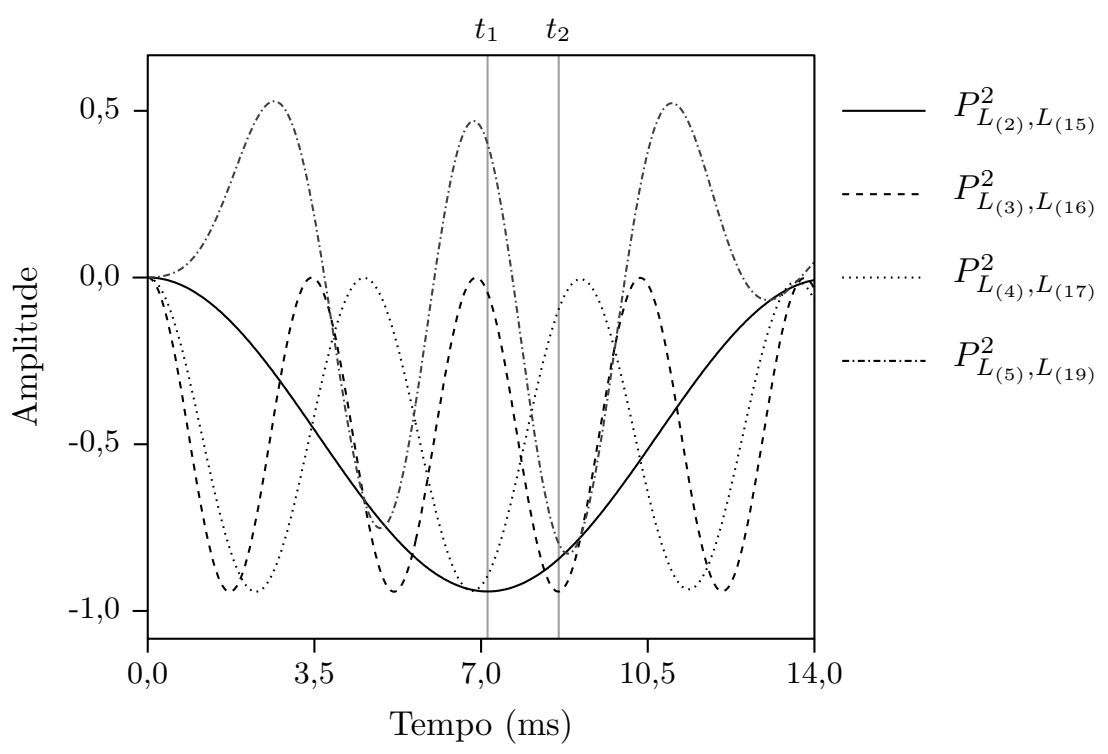

Figura 16: Gráfico da evolução da projeção dos estados de rank 0 nos estados de rank 2 devido à ação da hamiltoniana livre. Os tempos $t_{1}$ e $t_{2}$ aproximadamente maximizam a transferência entre as componentes $L_{(2)}, L_{(4)}$ e $L_{(3)}, L_{(5)}$, respectivamente.

lembrando que $\mathrm{U}_{f}(t)=e^{-i t\left(\mathrm{H}_{0}^{e f}+\mathrm{H}_{\text {int }}^{e f}\right) / \hbar}$. Calculando-se o projetor 3.57 para todas as componentes $\mathrm{T}_{l, 0}^{L}$, foi possível observar que a máxima transferência sempre ${ }^{2}$ ocorre para o rank $l=2$. A Figura 16 mostra o gráfico do projetor em função do tempo para as componentes em que ocorre a máxima transferência. Uma transferência total implica em $P= \pm 1$. O índice $L_{(i)}$ refere-se ao índice $L$ da $i$-ésima coluna da Tabela 1 . Foram escolhidos os tempos de evolução livre $t_{1}$ e $t_{2}$ indicados na parte superior do gráfico. Com $t_{1}=7,14 \mathrm{~ms}$, maximiza-se aproximadamente a transferência das componentes de índice $L_{(2)}$ e $L_{(4)}$. Com $t_{2}=8,93 \mathrm{~ms}$, maximiza-se a transferência das componentes de índice $L_{(3)}$ e $L_{(5)}$.

Dessa forma, a simulação da tomografia das coerências de ordem 0 foi feita aplicando-se primeiramente somente os pulsos não-seletivos sobre o estado de superposição. Esse procedimento forneceu as equações do sistema (3.44) que contêm informação sobre as componentes de rank $l>0$ e coerência $m^{\prime}=0$. Em seguida, o mesmo procedimento foi repetido com a única diferença de que, antes da aplicação dos pulsos, o sistema foi deixado evoluir sob a hamiltoniana não-perturbada durante os tempos $t_{1}$ e $t_{2}$. Esse procedimento resultou no sistema de equações (3.55). Como a transferência máxima foi para as componentes de rank $l=2$, foi utilizado o ângulo de nutação de $\pi / 4$, (vide Tabela 5). Portanto, os 20 elementos de ordem 0 foram obtidos das $12 \times 3=36$ equações do sistema (3.44) usando os ângulos da Tabela 5 mais $12 \times 2=24$ equações do sistema 3.55 usando o ângulo $\theta=\pi / 4$ e os tempos $t_{1}$ e $t_{2}$, to-

\footnotetext{
${ }^{2}$ No caso de 3 spins $1 / 2$.
} 
a)

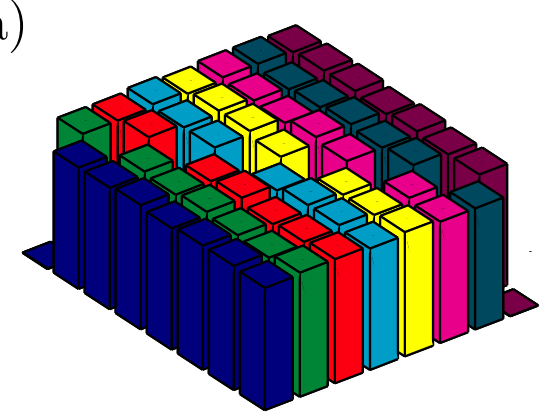

b)

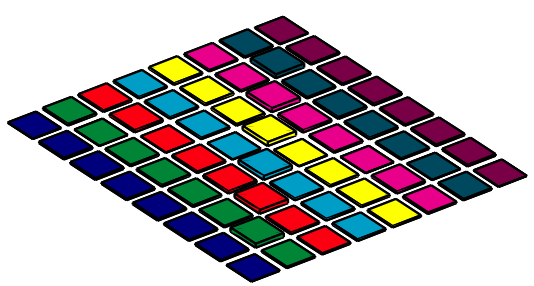

Figura 17: Simulação do método de tomografia para um sistema de 3 spins $1 / 2$ homonucleares. (a) Tomografia do estado correspondente à superposição $|\Psi\rangle=\frac{1}{2 \sqrt{2}} \bigotimes_{i=1}^{3}\left(|0\rangle_{i}+|1\rangle_{i}\right)$. (b) Desvio absoluto com relação à matriz de desvio dada pela Equação (3.51). Todos os pulsos de radiofreqüência foram aplicados com um erro sistemático de $5 \%$ no ângulo de nutação.

talizando 60 equações. O resultado da tomografia encontra-se na Figura 17(a). A Figura 17(b) mostra o desvio com relação ao estado original (3.51). O máximo desvio obtido para esse caso foi de $3 \%$.

\subsubsection{Sistemas de Spins Heteronucleares Acoplados}

Para sistemas de spins de espécies diferentes é possível obter uma expressão mais geral que a obtida na Equação 3.38. Isso porque é possível aplicar, simultaneamente, ângulos de nutação diferentes para cada conjunto de espécie nuclear e ainda assim manter a não-seletividade, conforme exposto pelo propagador do pulso (3.15). Este portanto é o caso mais geral, em que um número arbitrário de espécies nucleares com um número qualquer de núcleos de cada espécie e de qualquer spin interagem entre si para formar o operador densidade do sistema. Para aproveitar essa propriedade pode ser mais conveniente definir uma base mista, formada pelo produto dos tensores irredutíveis:

$$
\mathrm{T}_{l_{[1]}, m_{[1]}}^{L_{[1]}} \otimes \mathrm{T}_{l_{[2]}, m_{[2]}}^{L_{2]}} \otimes \ldots \otimes \mathrm{T}_{l_{\left[N_{s}\right]}, m_{\left[N_{s}\right]}}^{L_{[S]}}
$$

Cada termo $[i]$ refere-se à base de operadores $\mathrm{T}_{l m}^{L}$ que expande o espaço de operadores da $i$-ésima espécie nuclear. Para exemplificar essa abordagem considere o caso de apenas duas espécies nucleares distintas. O operador densidade do sistema, expandido na base (3.58) é escrito como:

$$
\rho=\sum_{r_{1}, r_{2}} a_{r_{1} r_{2}} \mathrm{~T}_{r_{1}} \otimes \mathrm{T}_{r_{2}}
$$

O índice $r_{i}$ é uma notação compacta para $L_{[i]}, l_{[i]}, m_{[i]}$. 
Aplicando o mesmo desenvolvimento da Seção 3.2, encontra-se que o efeito do propagador (3.15) sobre $\rho$ é dado por:

$$
\tilde{\rho}=\sum_{r_{1}, r_{2}} a_{r_{1} r_{2}} \sum_{m_{[1]}^{\prime}, m_{[2]}^{\prime}}\left(g_{m_{[1]}^{\prime}, m_{[1]}}^{L_{[1]}} \mathrm{T}_{l_{[1]}, m_{[1]}^{\prime}}^{L_{[1]}}\right) \otimes\left(g_{m_{[2]}^{\prime}, m_{[2]}}^{L_{[2]}} \mathrm{T}_{l_{[2]}, m_{[2]}^{\prime}}^{L_{[2]}}\right) .
$$

O termo $g_{m_{[i]}^{\prime}, m_{[i]}}^{L_{[i]}}$ é uma abreviação de $e^{i\left(m_{i}-m_{i}^{\prime}\right)\left(\phi_{i}-\frac{\pi}{2}\right)} d_{m_{i}^{\prime}, m_{i}}^{l_{i}}\left(-\theta_{i}\right)$. Note que os coeficientes $g$ ficam em função de índices com linha e sem linha e, portanto, fazem a conexão entre os coeficientes da expansão $a_{r_{1} r_{2}}$ e os tensores irredutíveis $\mathrm{T}_{l_{[i]}, m_{[i]}^{\prime}}^{L_{[i]}}$. Assim, pode-se escolher combinações de ângulos $\theta_{i}$ e $\phi_{i}$ para que tensores de primeira ordem $\left(m_{[i]}^{\prime}=1\right)$, os quais produzem magnetização transversal, contribuam para os coeficientes $a_{r_{1} r_{2}}$ escolhidos.

Vale salientar que o procedimento de TEQ usando somente um ângulo de nutação $\theta$ por pulso não-seletivo também pode ser usado para o caso heteronuclear da mesma forma que para o homonuclear. A exposição feita nesta sub-seção apenas teve como objetivo utilizar a possibilidade de aplicar os pulsos não-seletivos mais gerais possíveis. Não será feito o desenvolvimento subseqüente da Equação (3.60), pois a complexidade de um sistema heteronuclear geral começa a envolver muitas variáveis, sendo que o tratamento via um método analítico começa a perder o seu apelo. Para sistemas de muitos núcleos acoplados, o ideal é empregar técnicas de espectroscopia bi ou multi-dimensional como a proposta no trabalho de Das et al. [47] e comentada na introdução deste capítulo. 


\section{Implementação Experimental do Método de TEQ}

Neste capítulo são expostos os principais resultados experimentais obtidos neste trabalho. Ênfase é dada na descrição dos procedimentos e métodos utilizados na obtenção dos resultados. Embora muitos dos procedimentos propostos sejam grandemente influenciados pelo tipo de equipamento e amostra utilizados especificamente neste trabalho, também são feitas generalizações para outros sistemas experimentais.

A seção 4.1 contém uma descrição do cristal líquido utilizado como amostra para computação quântica. Na seção 4.2 são descritas as implementações das seqüências de pulsos que produzem as operações lógicas sobre o sistema de spins juntamente com os pulsos responsáveis pelo processo de tomografia da matriz densidade. Na seção 4.3 são expostos os experimentos que comprovam a eficiência do método de tomografia descrito no capítulo 3 . Por fim, a seção 4.4 contém aplicações do método de tomografia através da leitura dos resultados da criação de estados pseudo-puros, aplicação de portas lógicas e relaxação dos estados quânticos.

\subsection{A Amostra de Cristal Líquido}

A amostra utilizada neste trabalho é formada por íons de sódio diluídos em uma matriz líquido-cristalina. A orientação das moléculas do cristal líquido $(C L)$ possibilita a produção de um gradiente de campo elétrico não nulo no espaço intermolecular e com uma direção preferencial com relação ao campo magnético principal. Assim, conforme discutido na sub-seção 2.1.2, os núcleos de sódio, por possuírem spin 3/2, interagem através do acoplamento quadrupolar que em perturbação de $1^{a}$ ordem é descrito pela hamiltoniana (2.53). Neste ponto será apresentada uma breve discussão sobre as características físico-químicas da amostra de cristal líquido. Para uma descrição mais completa de experimentos de RMN em cristais líquidos vide [55]. 
a) Fase nemática

$y$

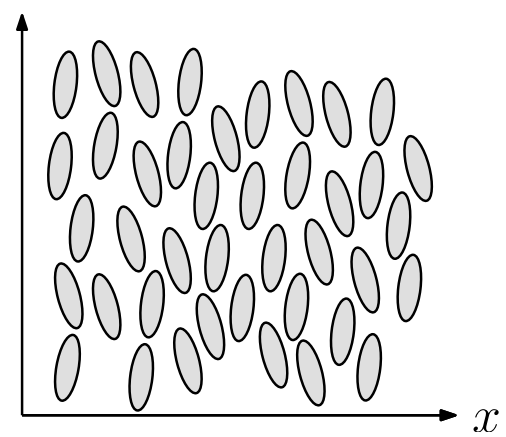

b) Fase esmética $\mathrm{C}$

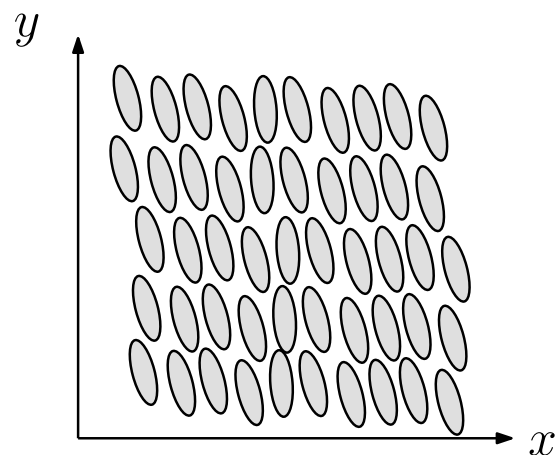

Figura 18: Exemplos de mesofases de um cristal líquido termotrópico. a) Na fase nemática há ordenamento direcional, direção y nesse caso, mas não há ordenamento translacional. b) $\mathrm{Na}$ fase esmética $\mathrm{C}$ há ordenamento direcional, levemente inclinado com relação à direção $y, \mathrm{e}$ translacional ao longo da direção $x$. O eixo $x$ representa qualquer direção pertencente ao plano perpendicular a $y$.

Os cristais líquidos, dependendo da sua composição e temperatura, apresentam fases intermediárias entre o ordenamento bem definido das moléculas em uma fase sólida cristalina e a ausência de ordenamento em uma fase líquida. Por esse motivo essas fases também são chamadas de mesofases. Os graus de liberdade comumente utilizados para descrever o ordenamento do CL são os graus de liberdade translacional e direcional das moléculas.

Classificam-se os cristais líquidos em dois grupos: os termotrópicos e os liotrópicos. Os CL's termotrópicos foram historicamente os primeiros a serem estudados e se caracterizam por possuírem uma composição química bem determinada, sendo que as transições de fase são obtidas exclusivamente através da variação da temperatura. As mesofases mais comuns dos CL's termotrópicos são a fase nemática e esmética. Na fase nemática, da mesma forma que em líquidos, não existe ordenamento translacional e a variação da posição das moléculas ao longo do tempo não possui nenhuma direção preferencial. No entanto, diferentemente dos líquidos, existe um certo grau de ordenamento direcional em que as moléculas possuem uma orientação média não-nula ao longo do tempo e ao longo do espaço para grandes distâncias comparadas à dimensão das moléculas. A Figura 18 a) ilustra a fase nemática. A fase esmética se caracteriza pelo ordenamento adicional dos graus de liberdade translacionais, em que as moléculas se movimentam preferencialmente ao longo de planos específicos. A Figura 18,b) ilustra a chamada fase esmética $\mathrm{C}$, em que a direção média das moléculas forma um ângulo oblíquo com o plano de translação.

Os cristais líquidos liotrópicos possuem as suas fases dependentes tanto da temperatura quanto da concentração das diversas substâncias que constituem o material. Normalmente, es- 
ses CL's são compostos por uma molécula anfifílica ${ }^{1}$ formada por uma cabeça polar e uma cauda apolar constituída por alguma cadeia de hidrocarbonetos. Essas moléculas são diluídas em algum solvente cuja polaridade irá determinar para que lado a cabeça e a cauda das moléculas irão se orientar nas estruturas formadas no CL. Assumindo um solvente polar como a água por exemplo, tem-se que para baixas concentrações as moléculas anfifílicas distribuem-se aleatoriamente como em qualquer solução não-saturada. A partir de uma concentração crítica as moléculas começam a se organizar em aglomerados chamados de micelas, nos quais as cabeças das moléculas ficam voltadas para o solvente e as caudas ficam distanciadas do solvente no interior das micelas. Conforme a concentração aumenta, estruturas mais compactas são formadas. Ocasionalmente, formam-se micelas esféricas organizadas em redes cúbicas². Com o aumento da concentração as micelas assumem o formato de bastões que se organizam em redes hexagonais. Por fim, é possível alcançar uma estrutura lamelar em que as moléculas se orientam em camadas formando planos separados por água.

Dependendo das substâncias utilizadas, é possível formar micelas não esféricas ou estruturas anisotrópicas mais gerais, as quais podem assumir um certo grau de orientação. Nesse caso, existe uma semelhança com a fase nemática dos CL's termotrópicos, com a diferença de que a orientação é atribuída a estruturas mais complexas ao invés de moléculas individuais. Por esse motivo, CL's que exibem essa propriedade são chamados de cristais líquidos liotrópicos nemáticos. Esse é o tipo de cristal líquido utilizado neste trabalho.

A amostra utilizada possui como molécula anfifílica o detergente aniônico ${ }^{3}$ dodecil sulfato de sódio (DSS) $\mathrm{CH}_{3}\left(\mathrm{CH}_{2}\right)_{11} \mathrm{OSO}_{3} \mathrm{Na}$. Como solvente polar foi empregada água deuterada $\mathrm{D}_{2} \mathrm{O}$. Além disso, utilizou-se uma pequena quantidade de decanol (DeOH) $\mathrm{CH}_{3}\left(\mathrm{CH}_{2}\right)_{9} \mathrm{OH}$. As concentrações utilizadas foram $21,3 \%$ de DSS, 75,2\% de $\mathrm{D}_{2} \mathrm{O}$ e 3,6\% de DeOH. Essas concentrações, bem como o procedimento de preparação das amostras são muito semelhantes aos contidos na tese de doutorado do Dr. Bonk [7]. A diferença com relação ao trabalho anterior foi o recipiente usado para acondicionar a amostra e a sonda de RMN utilizada para realizar os experimentos. Neste trabalho optou-se por empregar a sonda padrão do espectrômetro Varian Inova, a qual por possuir uma bobina com desenho solenoidal apresenta grande eficiência na produção de campos de RF intensos. Esse requisito é importante para que os pulsos não-seletivos exigidos pelo método de tomografia sejam satisfatoriamente implementados. No entanto, como conseqüência do compromisso entre intensidade e homogeneidade, o campo $B_{1}$ é significativamente não uniforme no volume compreendido pela bobina.

\footnotetext{
${ }^{1} \mathrm{Ou}$ seja, possui uma parte hidrofílica e uma parte hidrofóbica.

${ }^{2}$ Essas redes são muito menos rígidas que em cristais sólidos, permitindo um razoável grau de mobilidade translacional para as micelas.

${ }^{3}$ A parte orgânica situa-se no ânion da molécula.
} 


\section{Corte Longitudinal}

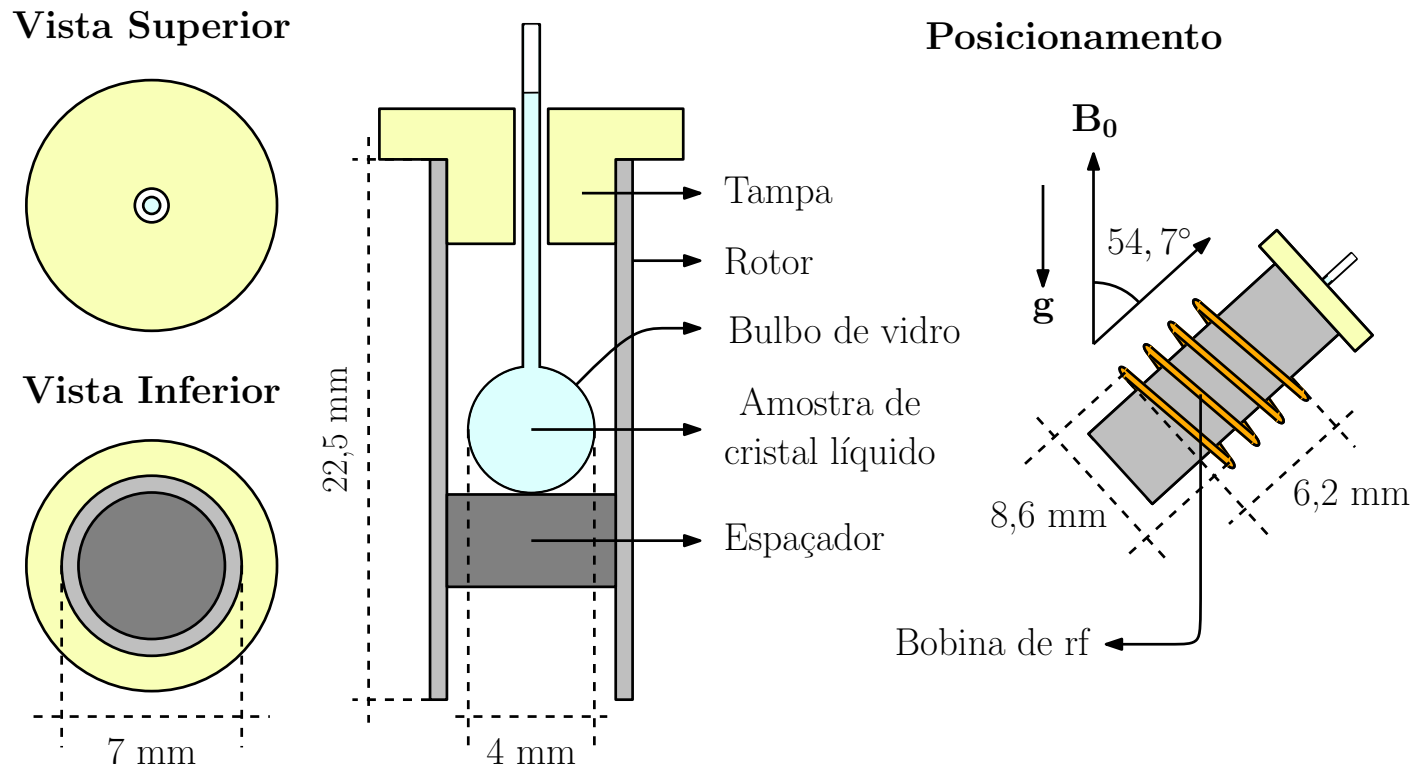

Figura 19: Dimensões e geometria do posicionamento da amostra de cristal líquido. A espessura da parede de vidro do bulbo é de aproximadamente $0,2 \mathrm{~mm}$. g é o vetor aceleração gravitacional. As proporções do desenho não são rigorosamente iguais às dos objetos reais e as cores não correspondem necessariamente às cores reais dos objetos.

Por esse motivo, utilizou-se um recipiente esférico de vidro (bulbo) com dimensões reduzidas. Mesmo com essa escolha, ainda assim obteve-se uma boa relação sinal-ruído justamente devido à eficiência da bobina. Além disso, a escolha de uma geometria esférica para a amostra facilita a homogeneização de $B_{0}$ conforme discutido em [56]. Essa homogeneidade é importante para que efeitos de relaxação possam ser desprezados ao longo da aplicação dos pulsos relativamente mais longos que geram as operações de CQ. Para posicionar o bulbo dentro da bobina utilizouse um rotor padrão empregado para rotação de amostras sólidas em torno do ângulo mágicot Confeccionou-se uma tampa de teflon para o rotor com um furo central atravessando-a do topo ao fundo. Esse furo foi feito com um diâmetro levemente superior ao diâmetro externo do pescoço do bulbo. Com isso, foi possível manter o recipiente razoavelmente centralizado na direção transversal do rotor e ainda assim permitir que o bulbo corresse livremente em função da posição fixada pelo espaçador contido no fundo do rotor. A Figura 19 contém as dimensões e a geometria do posicionamento da amostra.

Devido ao tamanho reduzido do pescoço do bulbo de vidro e à grande viscosidade da amostra de cristal líquido, foi razoavelmente difícil introduzir a amostra dentro do recipiente. Portanto é interessante comentar o procedimento adotado para realizar essa tarefa. Primeiramente, retira-se uma pequena quantidade de amostra de dentro do recipiente de preparação utilizando-

\footnotetext{
${ }^{4} \mathrm{~A}$ amostra foi mantida estática. O motivo da utilização do rotor é o aproveitamento da configuração padrão da sonda.
} 
se uma seringa simples. O diâmetro da agulha da seringa deve ser o mais largo possível para facilitar o escoamento, mas que ainda assim permita a introdução da mesma dentro do pescoço do bulbo. A inserção do CL é feita encostando-se a ponta da agulha no fundo do bulbo e aplicando-se uma leve pressão sobre o êmbolo da seringa. À medida que o CL é expelido pela agulha o mesmo começa a preencher de forma errática o fundo do bulbo. Por isso, para evitar a formação de bolhas é interessante ir rodando levemente a seringa de forma a tentar homogeneizar o preenchimento do bulbo. É interessante também que o preenchimento seja feito até uma altura grande do pescoço para evitar que a interface da amostra com o ar esteja na região de maior sensibilidade da bobina, pois nessa região de interface a variação de suscetibilidade magnética é intensa, produzindo gradientes de campo magnético que contribuem para uma maior relaxação transversal. Caso algumas bolhas permaneçam no interior do bulbo, pode-se centrifugar a amostra de forma a expelir as bolhas. No entanto, as bolhas não devem ser muito grandes, caso contrário o CL não preenche todo o interior do bulbo e parte do pescoço.

Voltando às propriedades do cristal líquido empregado, é interessante mencionar o processo de orientação das micelas da fase liotrópica nemática na presença do campo magnético, justificando a escolha desse tipo específico de material para os nossos experimentos de CQ. De fato, a principal justificativa para a utilização de meios líquidos cristalinos no estudo de núcleos quadrupolares está na combinação entre anisotrópica e mobilidade das mesofases. Ou seja, um ambiente molecular anisotrópico restringe os graus de liberdade de movimento dos átomos observados, íons de sódio no caso deste trabalho, fazendo com que interações que não possuam contribuições isotrópicas não sejam anuladas pelo movimento molecular. Ao mesmo tempo, a mobilidade ainda presente nos interstícios do CL permite que a interação dipolar seja bastante atenuada se comparado aos sistemas sólidos. Ainda assim, devido ao grande momento dipolar nuclear do hidrogênio da água, ocorre um alargamento das linhas espectrais do sódio da ordem do acoplamento quadrupolar. Com o intuito de aumentar a resolução espectral de forma a resolver satisfatoriamente a interação quadrupolar é que utiliza-se água deuterada como solvente no lugar da água comum. Como o núcleo de deutério possui um momento de dipolo aproximadamente 6,5 vezes menor que do próton, o alargamento dipolar é bastante reduzido. Poder-se-ia pensar que seria interessante usar moléculas de DSS com todos os hidrogênios marcados com deutério. No entanto, conforme ilustrado na figura 5 da ref. [55], os íons de sódio estão predominantemente presentes na interface das cabeças polares do DSS com o solvente. A cabeça polar do DSS é formada pelo grupo $\mathrm{SO}_{3}^{-}$cujos isótopos magnéticos possuem baixa abundância natural e pequeno momento dipolar, não produzindo portanto um acoplamento dipolar significativo. Somente uma pequena quantidade de sódio e água estão contidos ao longo da cadeia de hidrocarbonetos do DSS. 
As propriedades de ordenamento dos cristais líquidos podem ser propagadas para dimensões relativamente grandes da amostra. No entanto, no volume completo do material podem haver variações significativas das direções das orientações das micelas de forma tal que ocorra uma dispersão no valor do acoplamento quadrupolar. Nesse ponto, a presença do forte campo magnético principal $B_{0}$ apresenta o benéfico efeito de orientação das micelas ao longo da direção do campo. Esse efeito é possível pois a fase liotrópica nemática não possui uma interação entre as micelas forte o suficiente a ponto de evitar que o torque produzido pela interação do campo com o momento de dipolo da micela não mude a orientação da mesma. A principal interação que determina a direção de orientação do CL liotrópico nemático na ausência do campo é a interação com a superfície de contato com o recipiente. Portanto, um recipiente de vidro com formato esférico ou ovalado pode produzir, na ausência de campo, variações de direção do cristal bastante complexas. Um procedimento simples utilizado para verificar se alguma fase anisotrópica do CL liotrópico foi alcançada, é observando a atividade ótica da amostra via mudança do plano de polarização da luz medida com o auxílio de polarizadores cruzados [7]. A atividade ótica do CL não se deve a nenhum efeito de quiralidade 5 pois todos os compostos constituintes são aquirais. A mudança do plano de polarização se deve à propriedade de birrefringência do CL. A birrefringência é devida à anisotropia do cristal que faz com que as componentes dos raios luminosos com planos de polarização paralelos aos distintos eixos das micelas presenciem índices de refração distintos. Para uma descrição dos efeitos das interações de superfície e de campo magnético nas mesofases de CL's liotrópicos e o procedimento de medida através da atividade ótica, vide $[57,58]$.

A argumentação do parágrafo acima pôde ser corroborada pelas observações do comportamento da amostra no período de aplicação do campo magnético principal. Imediatamente antes de inserir a amostra dentro do magneto de RMN verificava-se a existência de atividade ótica através do uso dos polarizadores cruzados. Portanto, da discussão acima, conclui-se que alguma mesofase anisotrópica estava presente. Alguns minutos após a colocação da amostra dentro do magneto, o sinal de RMN apresentava linhas laterais bastante largas e muito pouco intensas indicando uma razoável dispersão de orientações das estruturas do CL. Repetindo-se a medida do sinal ao longo do tempo observava-se um gradual estreitamento e aumento de intensidade das linhas laterais até um ponto de estabilidade alcançado em um período de uma a duas horas. Durante todos os experimentos de RMN realizados nesse material a temperatura da amostra ficou em torno de $20^{\circ} \mathrm{C}$.

\footnotetext{
${ }^{5}$ Compostos quirais podem produzir mesofases chamadas de colestéricas que possuem interessantes propriedades de polarização da luz.
} 


\subsection{Seqüências de Pulsos e Procedimentos de Calibração}

Nesta seção são expostas as sequiências de pulsos e os procedimentos de calibração empregados para obter todos os resultados experimentais contidos neste trabalho. As seqüências de pulsos descritas nos capítulos anteriores não levam em conta as características e limitações do instrumental empregado. Portanto, são necessárias adaptações e modificações inerentes ao processo experimental e à especificidade do equipamento, as quais são discutidas aqui.

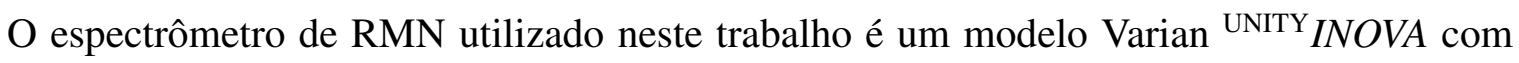
campo de 9,38 T e localizado no IFSC-USP. A configuração do sistema é otimizada para experimentos com amostras sólidas. Descrições de componentes específicos do equipamento serão feitas ao longo do seu aparecimento na seção.

A primeira etapa para a obtenção de seqüências robustas é a avaliação das características do campo magnético principal e do campo de rf produzidos pelo equipamento. O parâmetro mais importante relacionado ao campo principal é a sua homogeneidade. Para obter essa informação de forma bastante direta, foi feita a medida da largura da linha do núcleo de deutério em uma amostra pura de água deuterada (> 99\%) utilizando-se um pulso de excitação de $\pi / 20$. O volume e o posicionamento da amostra foram mantidos os mesmos o tanto quanto possível com relação a amostra de cristal líquide 6 . Utilizou-se a linha da água deuterada pura ao invés do deutério da própria amostra de CL para que o alargamento da linha fosse devido predominantemente à não-homogeneidade do campo. Após a realização do procedimento de shimming 7 foi possível obter uma largura de linha de $5,7 \mathrm{~Hz}$ a $50 \%, 71,4 \mathrm{~Hz}$ a $0,55 \%$ e 78,4 $\mathrm{Hz}$ a $0,11 \%$ da altura máxima do espectro. Adotando-se o procedimento padrão de associar a largura à meia altura do espectro, $\Delta v$, à variação do campo $\Delta B \approx \gamma \Delta v$ na região da amostra, encontra-se uma dispersão espacial de freqüência para o núcleo de sódio da amostra de CL de $\Delta v_{\mathrm{Na}}=\Delta \nu_{\mathrm{D}} \frac{\gamma_{\mathrm{Na}}}{\gamma_{D}} \approx 10 \mathrm{~Hz}$.

Para fazer-se a avaliação da não-homogeneidade do campo de rf, optou-se pela simulação do perfil de campo magnético produzido pela bobina de rf e pelo cálculo do desvio padrão do campo na região esférica da amostra ${ }^{8}$ A sonda utilizada, modelo VT CP/MAS de $7 \mathrm{~mm}$, possui uma bobina solenoidal de 4 espiras completas com diâmetro médio de 8,6 mm e comprimento longitudinal de $6,2 \mathrm{~mm}$. O eixo longitudinal do solenóide faz um ânguld $9^{9}$ de $54,7^{\circ}$ com relação à direção de $B_{0}$, vide Figura 19 . O diâmetro interno do bulbo corresponde a 3,6 mm. A simulação

\footnotetext{
${ }^{6}$ Acredita-se que essa condição foi alcançada devido ao eficiente arranjo descrito na seção 4.1 e na Figura 19

${ }^{7}$ Uniformização do campo magnético através da aplicação de campos estáticos com intensidade controlável.

${ }^{8}$ A região do pescoço do bulbo na Figura 19 foi desprezada devido ao seu volume relativamente pequeno.

${ }^{9}$ Utilizado para experimentos com rotação em torno do ângulo mágico.
} 
foi feita através do cálculo numérico do campo produzido por vários segmentos lineares de corrente ao longo do percurso do solenóide na região de $10^{6}$ pontos uniformemente distribuídos no volume da amostra. O desvio padrão relativo da amplitude e o desvio padrão da fase do campo foram respectivamente:

$$
\begin{aligned}
\frac{\sigma_{B_{r f}}}{\left\langle B_{r f}\right\rangle} & =3,5 \% \\
\sigma_{\phi_{r f}} & =1,4^{\mathrm{o}} .
\end{aligned}
$$

Para se ter uma maior segurança sobre a amplitude do campo de rf optou-se por usar um desvio padrão relativo de $5 \%$.

A implementação experimental dos SMP's foi feita utilizando-se o recurso de pulsos modulados do espectrômetro. Os pulsos são passados para o espectrômetro através de um arquivo escrito em código ASCII e com extensão .RF. Para uma descrição mais completa vide a seção 2.10 de [59] e a seção 16.2 de [60]. Esse arquivo deve conter 3 colunas representando a fase, a amplitude e a duração, nessa ordem. Cada linha corresponde a um segmento do pulso em que os 3 parâmetros são mantidos constantes. Uma característica importante do espectrômetro é que as fases de todos os pulsos, incluindo a coluna de fases dos arquivos .RF, são negativas com relação à definição usada em (2.92). Ou seja, deve-se aplicar a transformação $\phi_{k} \rightarrow-\phi_{k}$ caso aquela definição seja usada.

A coluna de fases deve ser informada em graus, sendo que o software aceita valores em ponto flutuante. O tamanho da variável, segundo especificações do fabricante [60], é um pouco menor que 10 bits, resultando em uma resolução na fase de 0,5 graus. Portanto, somando-se o erro devido à não homogeneidade do campo de rf obtido acima, obtém-se um erro total de aproximadamente 2 graus na fase de cada segmento.

A coluna de amplitudes também pode ser composta por números em ponto flutuante, com tamanho de 10 bits para essa variável [60]. Isso implica em uma resolução de $1 / 2^{10} \approx 0,1 \%$ na amplitude de cada segmento com relação a amplitude máxima do pulso. Nessa coluna, o que importa são os valores relativos de cada segmento, pois o software normaliza as amplitudes com relação ao valor mais alto e atribui esse valor à amplitude escolhida externamente na interface do programa com o usuário. Nessa interface, o parâmetro de controle da amplitude é chamado de tpwrm e pode ser visualizado no painel cinza inferior da Figura 20 mais adiante. O parâmetro tpwrm possui tamanho de 12 bits, implicando em uma resolução na amplitude de $1 / 4096 \approx 0.03$ em unidades de tpwrm. Com base nesses valores, percebe-se que mesmo considerando um tpwrm de 1/40 do valor máximo de 4096 e considerando os segmentos mais baixos como tendo $1 / 10$ do valor do segmento mais alto, ainda assim encontra-se um erro de no máximo $1 \%$ na 


\begin{tabular}{|c|c|c|c|c|c|}
\hline$\Delta \omega_{0}$ & $\Delta \phi$ & $\Delta \omega_{1}$ & \multicolumn{2}{|c|}{$\Delta \tau$} & $\Delta \omega_{Q}$ \\
\hline \hline \multirow{2}{*}{$10 \mathrm{~Hz}$} & \multirow{2}{*}{$2^{\circ}$} & \multirow{2}{*}{$0,05 \omega_{1}$} & $\tau<50 \mu \mathrm{s}$ & $0,3 \mu \mathrm{s}$ & \multirow{2}{*}{$70 \mathrm{~Hz}$} \\
\cline { 4 - 5 } & & $\tau>50 \mu \mathrm{s}$ & $0,004 \tau+0,1 \mu \mathrm{s}$ & \\
\hline
\end{tabular}

Tabela 6: Estimativas dos desvios experimentais dos parâmetros para cada segmento do pulso modulado. $\Delta \omega_{0}$ é o desvio da interação Zeeman, $\Delta \phi$ é o desvio de fase, $\Delta \omega_{1}$ é o desvio da amplitude, $\Delta \tau$ é o erro na duração e $\Delta \omega_{Q}$ é o desvio da interação quadrupolar.

amplitude de cada segmento. Consequentemente, a consideração do erro de $5 \%$ devido à não homogeneidade de rf já abrange as limitações de resolução do espectrômetro.

A coluna de tempos tem que ser composta somente por números inteiros entre 0 e 255 unidades, ou seja, os intervalos de tempo possuem tamanho de 8 bits. Isso implica que, atribuindose o valor de 255 unidades ao segmento mais longo, encontra-se uma resolução de 0,4\% com relação à duração desse segmento. No entanto, independentemente do tamanho da variável, o hardware do espectrômetro aceita um valor mínimo de 0,2 $\mu$ s por unidade. Com isso, para segmentos com até $50 \mu$ s o erro é de $0,2 \mu$ s. Acima disso o erro passa a ser $0,4 \%$ da duração do segmento. O parâmetro de interface que controla o tempo total do pulso chama-se pw, vide Figura 20. A resolução de pw é de $0,025 \mu$ s (vide $3^{a}$ coluna da Tabela 1 de [60]), o que não altera significativamente o erro já considerado. Para aumentar a margem de segurança optou-se por considerar um erro adicional de $0,1 \mu$ s ao tempo dos segmentos.

Por fim, o último erro a ser considerado no processo de otimização dos pulsos é o desvio do acoplamento quadrupolar. Para se ter uma idéia desse desvio, foi utilizada uma seqüência de spin-eco simples para obter o tempo de relaxação transversal $T_{2}$ sem a influência da não homogeneidade do campo, a qual já foi considerada acima. Pois, enquanto a seqüência de spinacho refocaliza a interação Zeeman o mesmo não ocorre com a interação quadrupolar. $\mathrm{O}$ valor de $T_{2}$ obtido da média entre o $T_{2}$ das duas linhas laterais foi de aproximadamente 4,5 ms (vide experimento de relaxação). Associando o desvio quadrupolar máximo à largura à meia altura da respectiva lorentziana obteve-se como resultado uma dispersão de aproximadamente $70 \mathrm{~Hz}$. Os erros encontrados para todas as variáveis encontram-se listados na tabela 6 .

Tendo sido feito o levantamento dos erros envolvidos no processo experimental de implementação dos pulsos modulados é possível obter os valores das variáveis $\Delta \Omega, \Delta \theta, \Delta \phi$ e $\Delta \beta$ através da aplicação dos desvios da tabela 6 . Essas variáveis são utilizadas no processo de otimização descrito na seção 2.2 .

Limitações de potência e duração dos pulsos também devem ser consideradas. Valores extremos desses parâmetros devem ser penalizados durante o processo de busca dos SMP's. Com relação à amplitude de rf, observou-se que para um pulso de excitação de $4 \mu$ s de duração, a 
resposta da magnetização obedecia uma função senoidal com bastante fidelidade para ângulos de nutação de até $90^{\circ}$. Para amplitudes maiores começava-se a observar um desvio do comportamento senoidal, indicando que algum processo de saturação ou deformação do campo de rf começava a ocorrer. Portanto, foi imposto o limite de $\omega_{1}=\frac{\pi}{8} 10^{6} \mathrm{rad} / \mathrm{s}$ para a amplitude de rf. Com relação à duração máxima de cada segmento, foi aplicado o limite entre 20 e $50 \mu$ s para evitar que efeitos de relaxação comprometessem a performance do pulso.

Uma característica intrínseca a qualquer hardware são os tempos de espera necessários para que cada instrução comece a ser executada. Esses delays devem ser considerados no planejamento da seqüência, pois a omissão dos mesmos pode implicar em um acúmulo significativo de erros ao final da operação. O espectrômetro utilizado, vide tabela 31 de [59], gera um pré-delay total de $1,7 \mu$ s antes de aplicar um pulso modulado. Para corrigir esse atraso, foi imposto no processo de otimização dos SMP's um tempo fixo de evolução livre ao final do pulso. Assim, instruções sub-seqüentes ao pulso modulado podem ser intercaladas por delays que levem em conta o tempo de evolução livre e descontem o atraso devido ao pré-delay do modulador. Foi escolhido um tempo de $3 \mu$ s, que é um pouco maior que o tempo de pré-delay. Foi utilizado esse tempo para garantir que possíveis atrasos das instruções subseqüentes possam também ser descontados. O código do programa de otimização dos SMP's encontra-se no apêndice A.2. Esse código não gera diretamente o arquivo de pulso .RF. O arquivo gerado é uma macro escrita na linguagem do software do espectrômetro. O software utilizado é o VNMR 6.1B e a linguagem da macro é a MAGICAL II [59]. Um exemplo de macro criada pelo programa de otimização dos SMP's encontra-se no apêndice A.3 e corresponde à porta lógica $\mathrm{CN}_{A}$ (NOT-controlada com controle em $A$ ) em um sistema de spin $3 / 2$.

Ao ser chamada na linha de comando do VNMR, a macro do SMP executa as seguintes tarefas. Primeiramente, ela atualiza o valor do acoplamento quadrupolar através da leitura das freqüências das linhas laterais do espectro na posição de máximo do módulo das respectivas linhas. Por isso é importante adquirir um espectro de referência imediatamente antes de rodar a macro. Com base no valor medido de $\omega_{Q}$, são calculados os tempos dos segmentos conforme discutido na seção 2.2. Os tempos são calculados para que cada unidade na coluna de tempo seja múltipla de $0,3 \mu \mathrm{s}$. É feita essa escolha pois além do tempo mínimo de 0,2 $\mu$ s existe uma resolução de $0,1 \mu$ s imposta pelo hardware. Portanto, $0,3 \mu$ s é o menor valor acima do tempo mínimo e dentro da resolução do espectrômetro. Além disso, os arredondamentos contidos nesse processo acarretam um erro já considerado na otimização dos SMP's, vide tabela 6 A outra atualização feita pela macro é do valor do parâmetro tpwrm que controla a amplitude do campo de rf do SMP. Para relacionar esse parâmetro com a amplitude encontrada pelo programa de otimização utiliza-se o coeficiente de proporcionalidade alpha, tal que $\omega_{1}=$ alpha $\cdot$ tpwrm. 
Esse coeficiente é obtido antes de se iniciar os experimentos utilizando-se uma rotina simples de calibração. Essa rotina consiste em escolher a duração do pulso de excitação de $90^{\circ}$ como sendo de $4 \mu$ s. Com esse tempo fixo é feita a aquisição do sinal para um conjunto de valores de tpwrm. Um programa de ajuste é então usado para obter o tpwrm ${ }_{M}$ que maximiza o sinal e portanto corresponde a uma rotação de $90^{\circ}$. Dessa medida, calcula-se o valor de alpha através da relação alpha $=10^{6} \pi /\left(8\right.$ tpwrm $\left._{M}\right)$. O programa de ajuste foi escrito em linguagem $\mathrm{C}$ para poder ser compilado no próprio computador ${ }^{10}$ que roda o software de controle do espectrômetro. Por fim, tendo feito os cálculos para a coluna de tempo e para o valor de tpwrm a macro gera o arquivo de pulso .RF correspondente à porta lógica desejada. Para a criação dos pulsos que geram os estados pseudo-puros o procedimento é análogo, com a única diferença de que são gerados 4 pulsos para se criar um estado pseudo-puro. Esses pulsos são aplicados em repetições distintas do experimento de forma a produzir o processo de média temporal descrito na seção 2.1 .

A Figura 20 mostra as janelas de interface do software VNMR. Existem três janelas principais. A janela cinza superior é o ambiente de linha de comando do software. A região clara no centro pode mostrar tanto o sinal de RMN adquirido quanto o diagrama da seqüência de pulsos. A janela cinza inferior contém uma lista dos principais parâmetros utilizados na seqüência. A programação do "computador quântico por RMN" é feita da seguinte maneira. Primeiramente atribui-se a um parâmetro chamado index o valor -1. Com esse valor, a seqüência de pulsos consiste somente em um pulso de excitação simples. Essa condição é usada tanto inicialmente para se encontrar o parâmetro alpha de calibração de amplitude quanto para obter o espectro de referência utilizado pelas macros de criação das operações quânticas. A Figura 21 mostra essa etapa de calibração. Portanto, uma vez determinado alpha e tendo sido adquirido o espectro de referência, roda-se a macro de criação do pseudo-puro. A macro atribui as amplitudes tpwrm1 a tpwrm4 e os tempos pw1 a pw4 aos respectivos SMP's que geram o estado via média tempora ${ }^{11}$. Em seguida rodam-se as macros das portas lógicas. Cada macro pede para que seja escolhida a posição na seqüência em que a porta será realizada. As posições vão de 5 a 7 , implicando, respectivamente, na aplicação da $1^{\mathrm{a}}$ a $3^{\mathrm{a}}$ posição após a criação do pseudo-puro. Também respectivamente as macros calculam as amplitudes de tpwrm5 a tpwrm7 e os tempos de pw5 a pw7. O parâmetro index controla até que etapa o algoritmo quântico será processado. Para index igual a 0 somente o estado pseudo-puro é implementado. Para index de 1 a 3 são aplicadas, respectivamente, as portas lógicas de 5 a 7 de forma acumulativa após o pseudo-puro. O diagrama da Figura 22,a) ilustra esse processo de programação. Na Figura 20 está mostrada na janela central a escolha de index $=3$, que nesse caso realiza primeiramente a operação

\footnotetext{
${ }^{10}$ Compilador gcc rodando em uma máquina Sun com sistema operacional Solaris 8.

${ }^{11}$ Optou-se por 4 médias para a criação do estado pseudo-puro devido à boa convergência apresentada pelo algoritmo de otimização.
} 


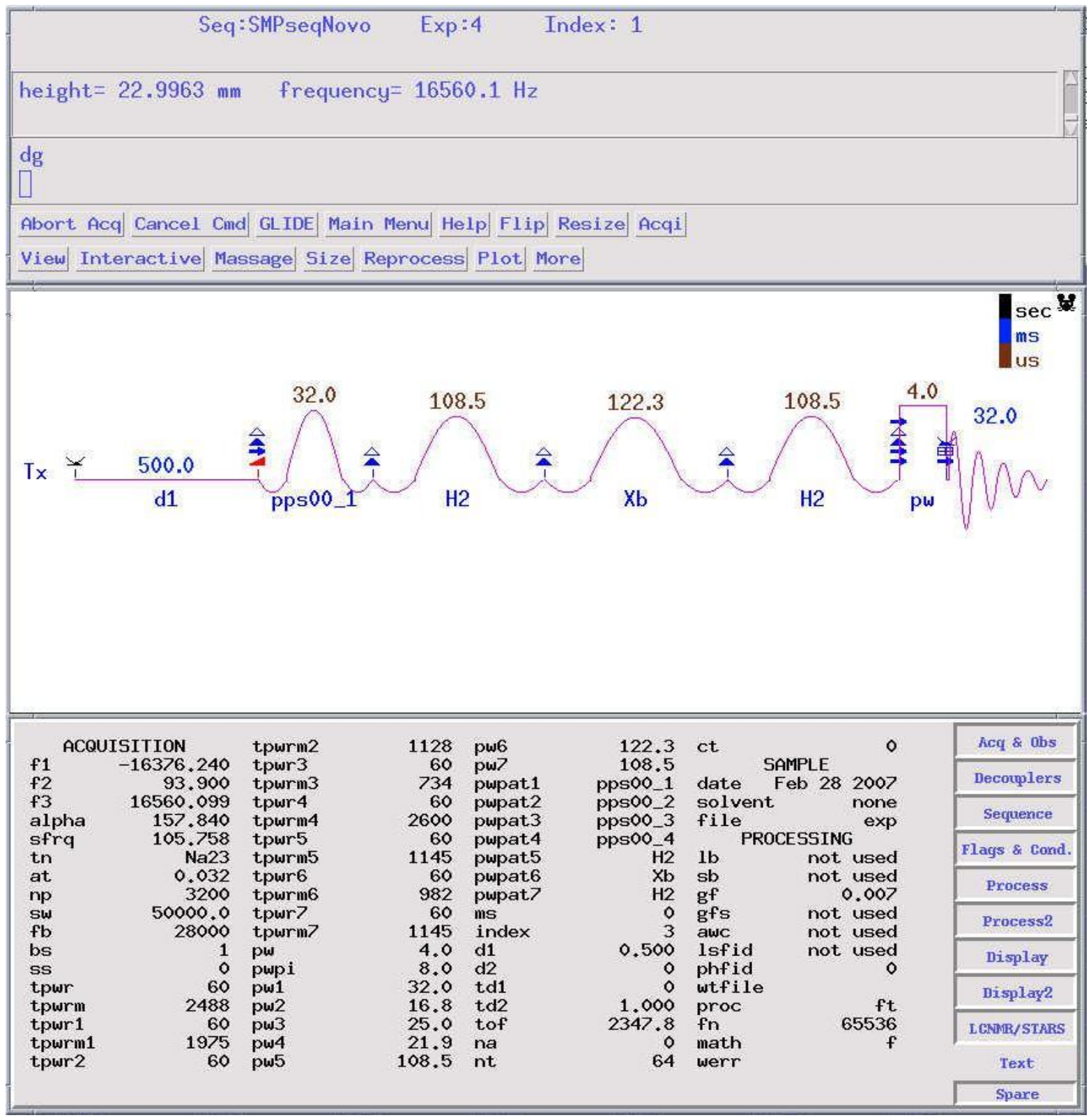

Figura 20: Snapshot do painel do software VNMR. Nessa configuração, o software apresenta três janelas de interface. A janela cinza superior contém a linha de comando onde o usuário executa os comandos específicos do VNMR e faz a chamada das macros de calibração e leitura. A janela clara central é onde são mostrados o sinal adquirido no(s) experimento(s) ou o diagrama da seqüência de pulsos utilizada. Nesse caso é mostrado o diagrama da implementação de três portas lógicas sobre um estado pseudo-puro. A janela cinza inferior contém os valores dos principais parâmetros de controle da seqüência. Os parâmetros citados nesta seção e que constam nessa janela são tpwrm, tpwrm1 a tpwrm7, pw, pw1 a pw7, pwpat1 a pwpat7, d1, nt, ms, index e alpha. 


\section{Calibração}

Módulo do espectro de referência
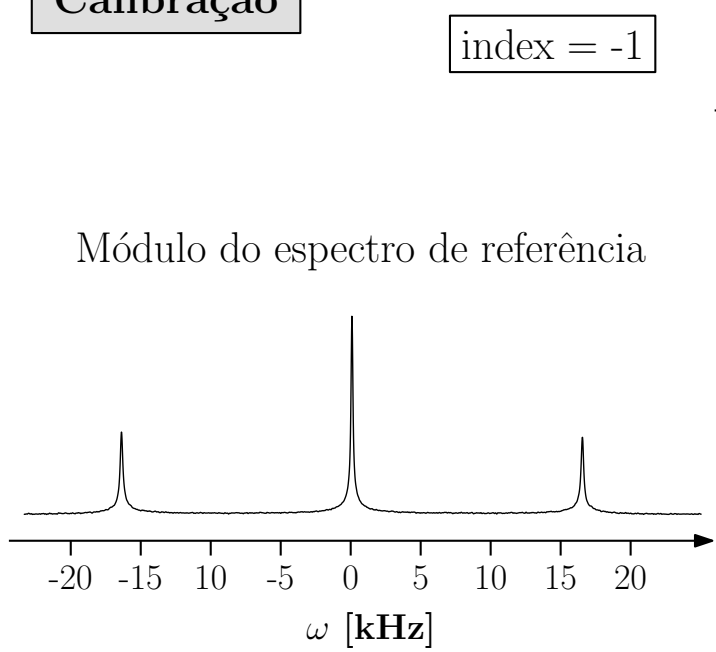

tpwrm,pw

d1

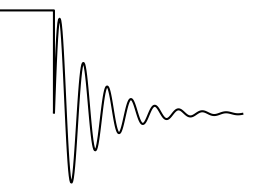

Módulo da Amplitude da linha central versus tpwrm

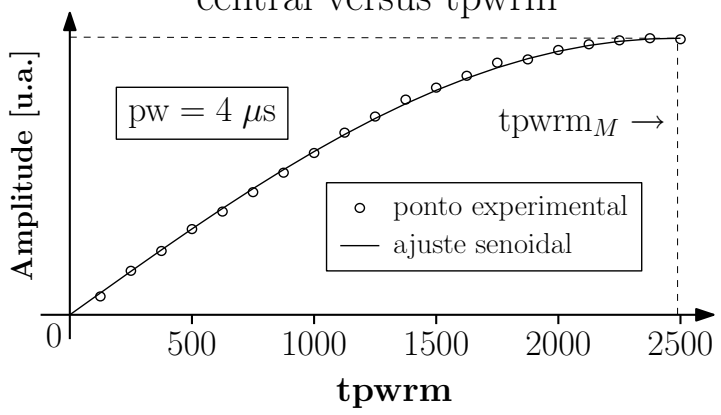

Figura 21: Diagrama da seqüência de pulsos e parâmetros relacionados à calibração do "computador quântico". A escolha de index $=-1$ corresponde à etapa de calibração, em que a seqüência se resume a um único pulso não-seletivo. $\mathrm{O}$ gráfico à esquerda corresponde ao módulo do espectro experimental utilizado para atualizar o valor de $\omega_{Q}$. O gráfico à direita também corresponde a medidas experimentais, sendo utilizado para obter o valor de $\operatorname{tpwrm}_{M} \mathrm{e}$ conseqüentemente o valor do parâmetro alpha para a calibração dos SMP's. O parâmetro d1 é o tempo de espera, ou repetição, para que a magnetização volte ao equilíbrio e uma nova média seja adquirida.

Hadamard nos dois q-bits sobre o estado $|00\rangle$, seguida de uma porta NOT no q-bit B e seguida novamente por uma Hadamard nos dois q-bits. Note que os nomes dos pulsos são atribuídos às variáveis pwpat na janela de parâmetros. Caso seja necessário aplicar mais do que três portas lógicas, basta aumentar o número de variáveis na janela de parâmetros e aumentar o número de pulsos na seqüência. Por último, no diagrama da seqüência, está mostrado o pulso de leitura responsável pelo processo de tomografia da matriz densidade resultante, o qual será discutido a seguir.

Conforme exposto pelas equações $(3.40)$, a sequiência de pulsos deve conter uma ciclagem de fases específica tanto para o pulso não-seletivo de tomografia quanto para a direção de detecção. O hardware do espectrômetro permite a aplicação de pulsos com fase arbitrária e com resolução de 0,25 graus. No entanto, o espectrômetro só aceita fases do detector que sejam múltiplas de 90 graus, e como pode ser visto na expressão para $\alpha$ em (3.40) são necessários ângulos mais gerais que esses para realizar a seleção de coerências, a menos da coerência de ordem 0 para spin 3/2. Esse problema pode ser resolvido facilmente observando-se que o estado inicial do sistema de spins, $\mathrm{I}_{z}$, é simétrico com relação à direção $z$. Portanto, o que realmente importa são as fases relativas entre os pulsos e a detecção, já que uma fase absoluta se propaga apenas como uma fase constante no espectro final. 


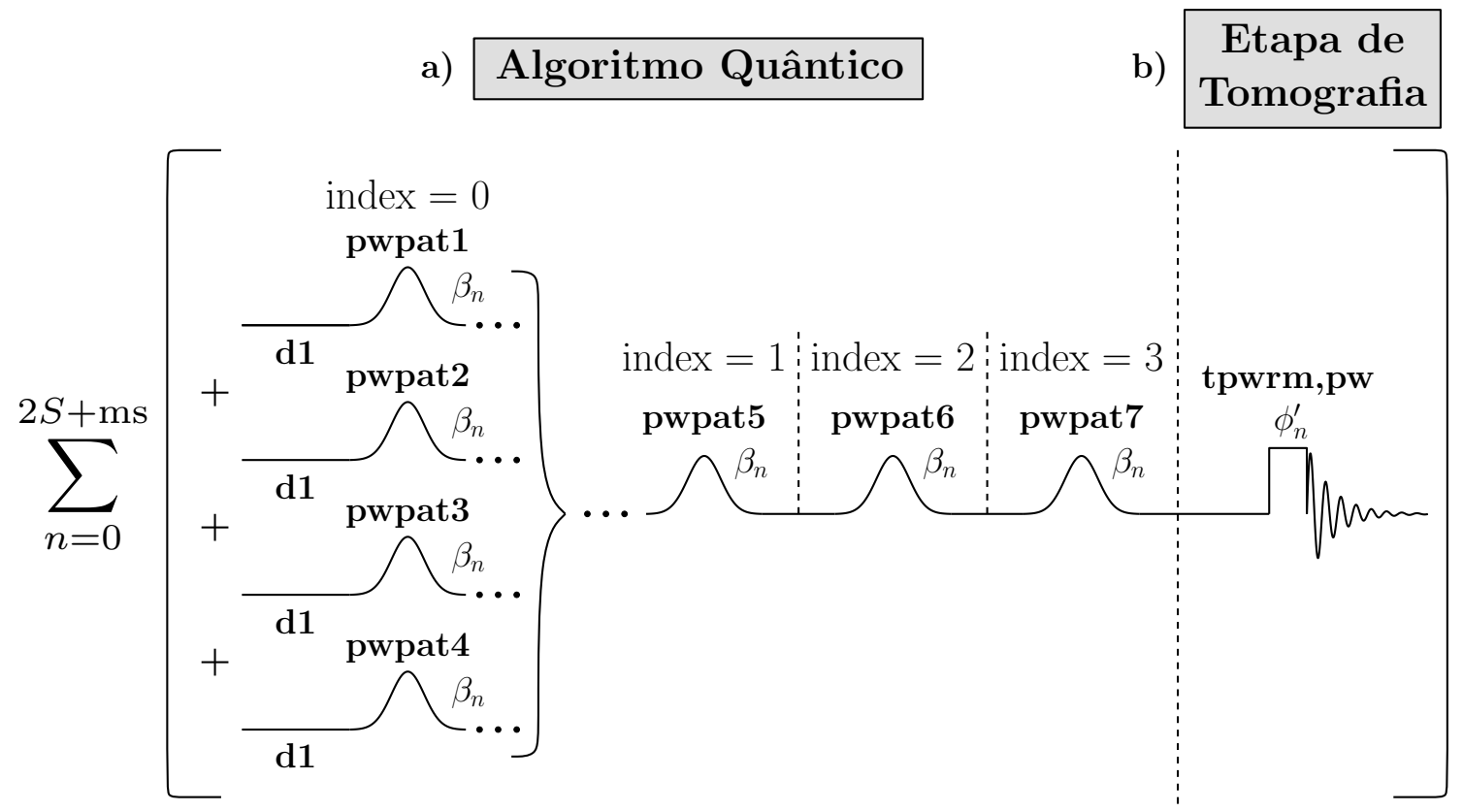

Figura 22: Diagrama da seqüência de pulsos e parâmetros relacionados à programação do "computador quântico". a) A implementação do algoritmo quântico é feita seqüencialmente através da escolha de index entre 0 e 3. b) A etapa de tomografia vem logo a seguir. Todos os pulsos possuem a fase variável ao longo das diferentes médias, de forma a realizar a seleção da coerência escolhida. Na figura, os pulsos com formato gaussiano simbolizam os pulsos modulados (SMP's) e os retangulares simbolizam pulsos não-seletivos. Todos os pulsos dessa figura e da anterior possuem uma ciclagem CYCLOPS que não está representada nos diagramas.

Dessa forma, optou-se por fixar o ângulo de detecção $\alpha_{n}^{\prime}$ em 0 graus, e com isso recalcular as fases dos pulsos de tomografia $\phi_{n}^{\prime}$ e as fases $\beta_{n}$ dos SMP's que geram os estados quânticos:

$$
\begin{gathered}
\phi_{n}^{\prime}=\phi_{n}+\alpha_{n}=2 \pi n m^{\prime} / N_{p}+\pi / 2, \\
\beta_{n}=0+\alpha_{n}=2 \pi n\left(m^{\prime}-1\right) / N_{p} .
\end{gathered}
$$

Em 4.1 foi tomada a soma com $\alpha_{n}$ ao invés da diferença, pois a multiplicação de uma fase positiva na magnetização da equação $(3.33)$ corresponde a uma fase negativa na detecção. $\mathrm{O}$ valor 0 à direita da primeira igualdade na segunda equação de (4.1) é para salientar que os SMP's são otimizados tomando-se a fase de 0 graus como referência. Pode-se pensar que esse procedimento de adicionar uma fase $\beta_{n}$ aos SMP's seja bastante enfadonha, pois seria necessário em princípio criar novos arquivos .RF com as fases transladadas de forma a realizar a seleção de coerências. No entanto, isso pode ser feito automaticamente no arquivo que descreve a programação da seqüência de pulsos do experimento. Até este ponto, não foi mencionado como que a seqüência de pulsos é programada. Não será descrito aqui esse processo de programação; para uma descrição completa do mesmo vide [59]. Neste trabalho são citados apenas alguns elementos do código de programação que consta no apêndice A.4. Esse é o código da seqüência 
de pulsos que realiza todos os experimentos expostos nesta tese, incluindo-se principalmente a etapa das operações quânticas, dos pulsos de tomografia e das evoluções livres para os estudos de dinâmica e relaxação expostos mais adiante. Voltando à questão de adicionar a fase $\beta_{n}$ aos SMP's, o espectrômetro permite escolher uma fase global dos pulsos, de tal forma que todas as fases dos segmentos dos pulsos modulados se somem a essa fase global. Dessa forma, não é necessária a criação de um pulso distinto para cada fase da tomografia.

Os parâmetros associados à etapa de tomografia são a amplitude tpwrm e o tempo pw do pulso não-seletivo, o número de médias nt e a ordem da coerência a ser selecionada ms. Todos eles constam na janela de parâmetros na Figura 20. Conforme discutido anteriormente, a amplitude tpwrm é escolhida de forma que o pulso de $90^{\circ}$ corresponda a pw igual a $4 \mu \mathrm{s}$. Assim, os diferentes ângulos de nutação necessários para maximizar a transferência de ranks específicos, vide Capítulo 3, são escolhidos variando-se proporcionalmente o valor de pw. Para realizar a seleção de uma coerência específica atribui-se ao parâmetro ms o valor da ordem $m^{\prime}$ dessa coerência. O parâmetro ms determina o valor dos incrementos de fase dos pulsos na seqüência do apêndice A.4 de acordo com as relações 4.1. O parâmetro nt determina o número de médias ou transientes realizados. Esse valor depende da ordem da coerência escolhida, pois o número mínimo de repetições $N_{p}$ depende de $m^{\prime}$, conforme estabelecido pela equação 3.40. Dessa forma, é necessário que nt seja múltiplo de $16 \times\left(2 S+m^{\prime}+1\right)$ para que o experimento seja corretamente realizado. A seqüência de pulsos contém uma rotina de verificação que impede que o experimento seja rodado caso nt não seja condizente com a coerência escolhida. $\mathrm{O}$ valor 16 aparece pois são necessárias 4 médias para gerar o estado pseudo-puro e para cada pulso aplicado na seqüência é gerada uma ciclagem de fases CYCLOPS, a qual consiste em 4 pulsos com fases alternadas de $90^{\circ}$. A utilização do CYCLOPS é para corrigir imperfeições no processo de detecção em quadratura, sendo que uma interessante descrição do seu funcionamento pode ser encontrada em [9]. O diagrama da Figura 22,b) ilustra o processo de tomografia. Pode-se pensar que o número de médias empregadas para se determinar uma única coerência seja muito grande. De fato, quanto maior o valor da coerência maior será o valor de nt. No entanto, no caso da amostra de cristal líquido estudada isso não foi um problema, pois o tempo de relaxação longitudinal $T_{1}$ era no máximo em torno de $0,05 \mathrm{~s}$. Portanto, com um tempo de repetição d 1 de $0,25 \mathrm{~s}$, a coerência mais alta, $m^{\prime}=3$, era tomografada em aproximadamente 30 segundos. Para amostras que possuam um tempo de relaxação $T_{1}$ bem mais longo pode-se optar pela seleção de coerências via média espacial com gradientes. Dessa forma não são necessárias médias adicionais para a tomografia, resultando em nt $=4 \times N_{p p s}$, em que $N_{p p s}$ é o número de médias necessárias para produzir os pseudo-puros. Ao final do apêndice A.4 está o código da seqüência de pulsos que implementa esse tipo de experimento. Esse código não chegou a ser 


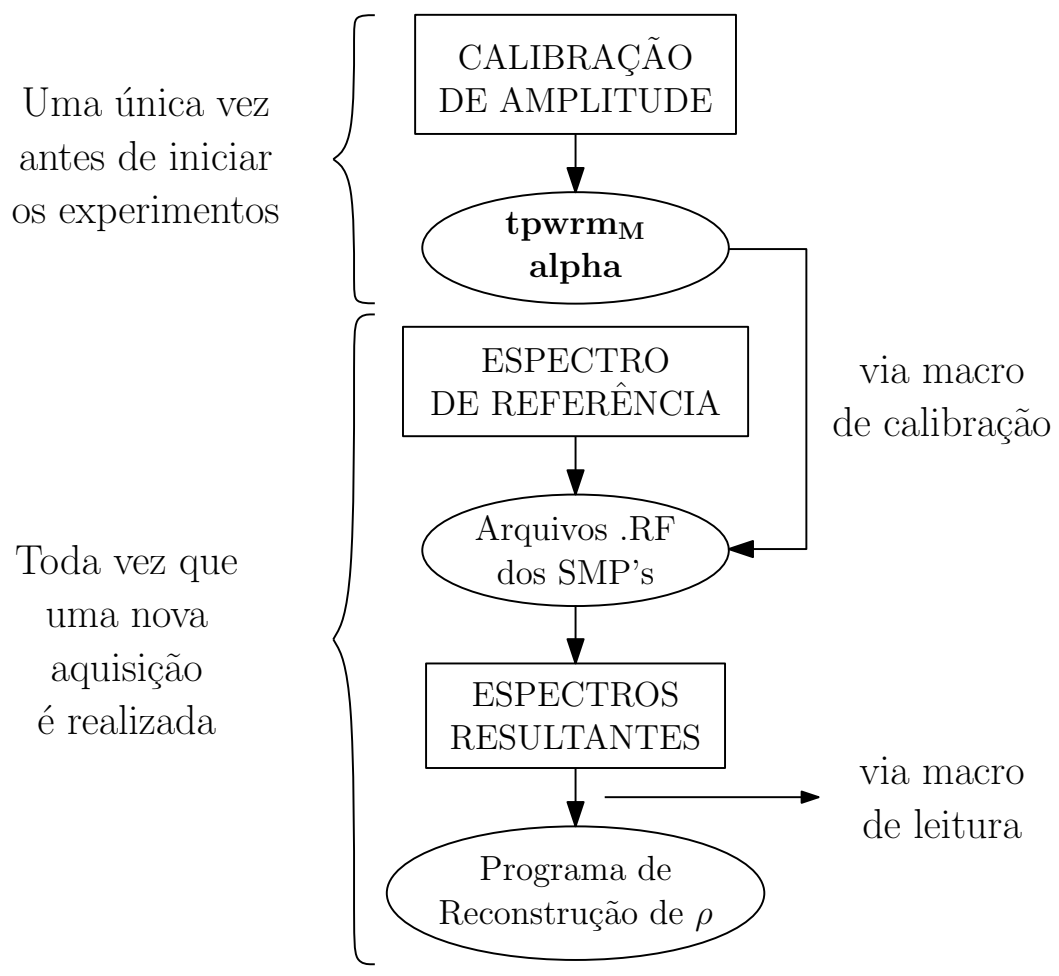

Figura 23: Fluxograma do processo de programação e tomografia realizados no espectrômetro de RMN.

testado, servindo apenas como uma diretriz para futuras implementações.

As amplitudes dos espectros obtidos são medidas e salvas em um arquivo. Para reconstruir a matriz densidade correspondente a essas medidas, foi desenvolvido um código em linguagem C que permite a obtenção da matriz densidade imediatamente após o término do experimento e no próprio computador que controla o espectrômetro. O código de reconstrução encontra-se no apêndice A.5 e o sistema de equações constante nesse código foi obtido do programa escrito em Maple listado no final desse mesmo apêndice. A construção dos dois programas é inteiramente baseada na teoria exposta no Capítulo 3. O código de reconstrução apresentado requisita que sejam informadas em um único arquivo as amplitudes de todas as coerências, sendo que cada coerência deve ser obtida com todos os ângulos ótimos de nutação. O apêndice A.6 contém a macro que programa a seqüência de experimentos necessários para a reconstrução da matriz densidade conforme exigido pelo código de reconstrução do apêndice A.5. Outra exigência do código de reconstrução é que o primeiro experimento programado pela macro seja um pulso de calibração, obtido escolhendo-se o parâmetro index = -1. O final do apêndice A.6 contém a macro que realiza a leitura das amplitudes obtidas e grava os resultados em um arquivo padrão do sistema chamado mark1d.out [59]. Esse arquivo pode ser lido diretamente pelo programa de reconstrução. O fluxograma da Figura 23 resume todo o processo de programação exposto nesta seção. 


\subsection{Validação experimental do método de TEQ}

Com o objetivo de encontrar procedimentos que ajudem a validar o método de tomografia proposto, serão apresentados nesta seção três experimentos usando-se a amostra de cristal líquido de DSS. O primeiro experimento consiste na criação de um estado inicial que possua todas as coerências com amplitudes aproximadamente iguais entre si. Nesse experimento, o interesse não está em obter um estado inicial específico com grande exatidão. O objetivo principal é utilizar o método de TEQ para descrever a dinâmica da matriz densidade e assim verificar se o resultado obtido concorda com o previsto pela hamiltoniana livre do sistema. Uma vez que a hamiltoniana livre é conhecida com grande exatidão, esse é um bom critério de validação. É utilizada essa abordagem pois a criação de estados quânticos pré-determinados envolve a implementação bem sucedida dos SMP's. De uma forma geral, experimentos que realizam a implementação de portas lógicas e algoritmos quânticos requerem um sucesso conjunto dos SMP's e do procedimento de tomografia, sendo difícil separar a contribuição de cada um. A próxima seção apresenta esse tipo de experimento.

O segundo experimento diz respeito à verificação da dependência da amplitude das coerências com o ângulo de nutação $\theta$ dos pulsos não-seletivos. Conforme discutido no capítulo 3, as funções de Wigner reduzidas $d_{1, m}^{l}(-\theta)$ determinam essa dependência. Para demonstrar experimentalmente esse resultado, foram otimizados SMP's para a criação de estados proporcionais aos operadores de polarização $\mathrm{T}_{l m}$. Variando-se o ângulo de nutação $\theta$ dos pulsos de tomografia foi possível verificar a dependência dos operadores $\mathrm{T}_{l m}$ com as respectivas funções de Wigner $d_{1, m}^{l}$.

Por fim, o terceiro experimento tem como objetivo a criação de coerências de até a ordem máxima para esse sistema, $m=3$, utilizando-se a sequiência de pulsos mais simples possível. Embora estados criados dessa maneira não correspondam a operações no contexto de computação quântica, esse é um procedimento que possibilita a obtenção de estados com uma maior confiança. Portanto, aplicando-se o método de tomografia a tais estados espera-se encontrar uma grande concordância com a previsão teórica.

Iniciando com o experimento de verificação da dinâmica das coerências, torna-se interessante reescrever aqui a forma da hamiltoniana quadrupolar em aproximação de $1^{a}$ ordem:

$$
\mathrm{H}_{Q}=\hbar \frac{\omega_{Q}}{6}\left[3 \mathrm{I}_{Z}^{2}-I(I+1) \mathbb{1}_{2 I+1}\right]
$$

Essa hamiltoniana deve descrever bastante bem a dinâmica do sistema, conforme pode ser visto no espectro da Figura 21. 


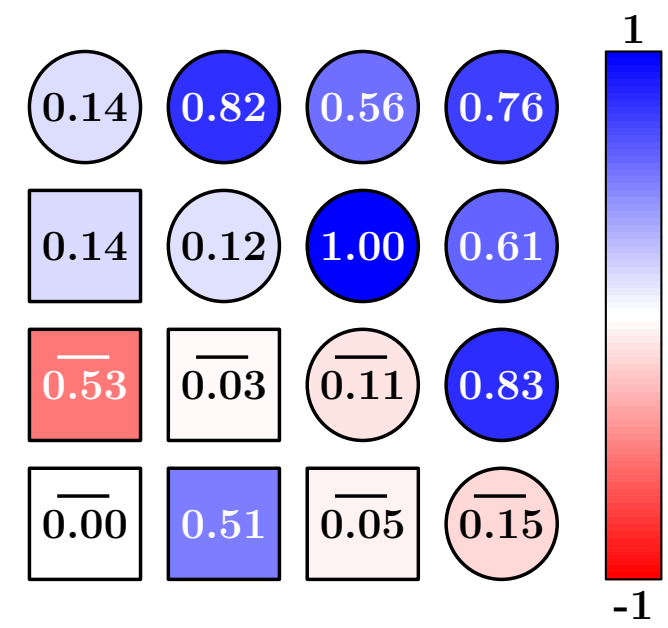

Figura 24: Estado de superposição em $\tau=0$ tomografado experimentalmente e criado para a observação da dinâmica dos elementos de $\rho$ sob a hamiltoniana quadrupolar $\mathrm{H}_{Q}$. Os círculos representam a parte real e os quadrados a parte imaginária dos respectivos elementos. Valores negativos são expressos com uma barra sobre os números. A escala de cor à direita relaciona-se com a cor de preenchimento dos círculos e quadrados. Todos os valores são normalizados com relação ao módulo do elemento de maior módulo.

Desses espectros, encontra-se um acoplamento quadrupolar de aproximadamente $16 \mathrm{kHz}$, que é muito menor que o acoplamento Zeeman de aproximadamente $100 \mathrm{MHz}$, justificando o uso da expressão 4.2 . .

Portanto, na condição de ressonância, os elementos da matriz densidade evoluem da forma $\rho_{i j}(t)=\rho_{i j}(0) e^{i \omega_{i j} t}$, em que $\omega_{i j}=[\mathbf{w}]_{i j}$, e a matriz de freqüências $\mathbf{w}$ para $I=3 / 2$ é dada por:

$$
\mathbf{w}=\left[\begin{array}{cccc}
0 & \omega_{Q} & \omega_{Q} & 0 \\
-\omega_{Q} & 0 & 0 & -\omega_{Q} \\
-\omega_{Q} & 0 & 0 & -\omega_{Q} \\
0 & \omega_{Q} & \omega_{Q} & 0
\end{array}\right]
$$

Para comparar a dinâmica de todos os elementos de $\rho$ com o previsto pela matriz w, otimizou-se um estado inicial via SMP's, que no estágio index $=0$ forneceu a matriz densidade da Figura 24. Embora esse estado possua coerências com fases diferentes entre si, os módulos das mesmas são bastante próximos, permitindo avaliar a evolução de todas as coerências utilizando-se esse mesmo estado inicial. A dinâmica do sistema ocorreu deixando-se o estado inicial evoluir livremente durante um tempo $\tau$ entre o final do último SMP e o começo do pulso de tomografia. A Figura 25 ilustra essa etapa. Esse tempo de evolução é implementado na sequiência de pulsos escolhendo-se o parâmetro na $=1$. O tempo $\tau$ é fixado pelo parâmetro d2. Ambos podem ser visualizados na janela inferior da Figura 20 e no código da sequiência de pulsos no Apêndice A.4. 


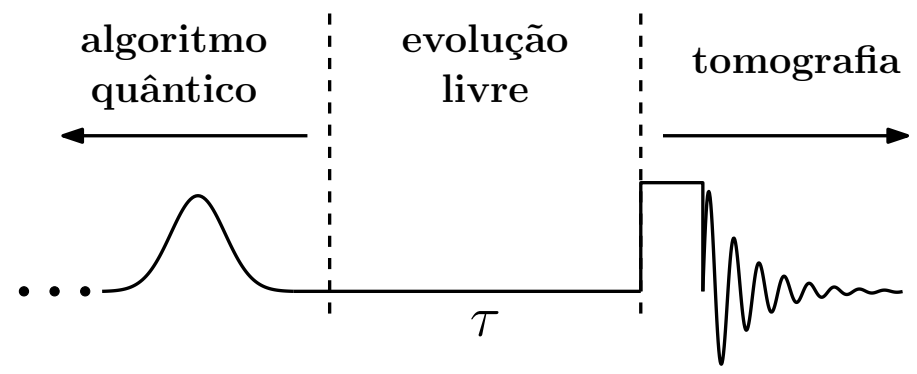

Figura 25: Diagrama da seqüência de pulsos contendo a etapa de evolução livre utilizada para estudar a dinâmica da matriz densidade. Nesse diagrama subentende-se que as fases $\beta_{n}$ e $\phi_{n}^{\prime}$ estão sendo aplicadas para os SMP's e pulsos de tomografia, respectivamente; vide Equação (4.1).

Uma questão importante que deve ser tratada nesta seção é a questão da fase inicial do sinal adquirido. De acordo com a discussão da seção anterior, o hardware do espectrômetro possui naturalmente tempos de atraso entre o instante de disparo de um comando e a efetivação desse mesmo comando. No caso do comando de aquisição existe a necessidade de impor um tempo de espera adicional entre a aplicação do último pulso de leitura e o início da detecção. Essa espera é necessária para que o sinal remanescente do último pulso não se misture ao sinal de resposta da amostra, causando efeitos de saturação e distorção nesse último. De fato, figuras como a 25 não explicitam esses tempos de espera entre o pulso de tomografia e o sinal medido. No entanto, esse tempo existe e pode chegar a dezenas de micro-segundos. Esse atraso na aquisição acarreta em uma fase inicial não nula no sinal temporal adquirido. Pode parecer que uma fase constante não altere a dinâmica dos elementos de $\rho$ medidos. No entanto, ao observar a forma da equação das amplitudes espectrais $\bar{S}_{i, i+1}$ em 3.48 percebe-se que um mesmo coeficiente $a_{l m}$ possuirá fases distintas para $\bar{S}_{i, i+1}$ 's distintos. Isso porque, cada $\bar{S}_{i, i+1}$ evolui com uma freqüência distinta. O efeito dessas fases na dinâmica dos elementos reconstruídos pode ser visualizado na simulação de tomografia da Figura 26. a). Nessa simulação foram usados dados similares aos descritos no experimento desta seção para reconstruir a dinâmica do elemento $\rho_{13}$. Foi empregado um tempo de espera de $5 \mu$ s antes da aquisição do sinal. Devido a isso, observa-se um saliente efeito de oscilação no módulo desse elemento. Como comparação, a Figura 26.b) mostra o resultado experimental da dinâmica do elemento $\rho_{13} \mathrm{em}$ um experimento em que não se tomou o cuidado de calibrar a fase inicial do sinal adquirido.

O processo de calibração baseado no espectro de referência e indicado na terceira etapa de cima para baixo do fluxograma da Figura 24 também atenta para a calibração da fase inicial. Na prática o que se faz é aplicar o comando de ajuste automático de correção de fase aph0 [59]. A macro de leitura do Apêndice A.6 realiza esse procedimento. Assim, após os procedimentos de calibração, obtiveram-se os resultados experimentais da Figura 27 para as coerências que 
a) Simulação da tomografia de $\rho_{13}$ com tempo de espera de $5 \mu \mathrm{s}$ b) Tomografia experimental de $\rho_{13}$ sem correção de fase

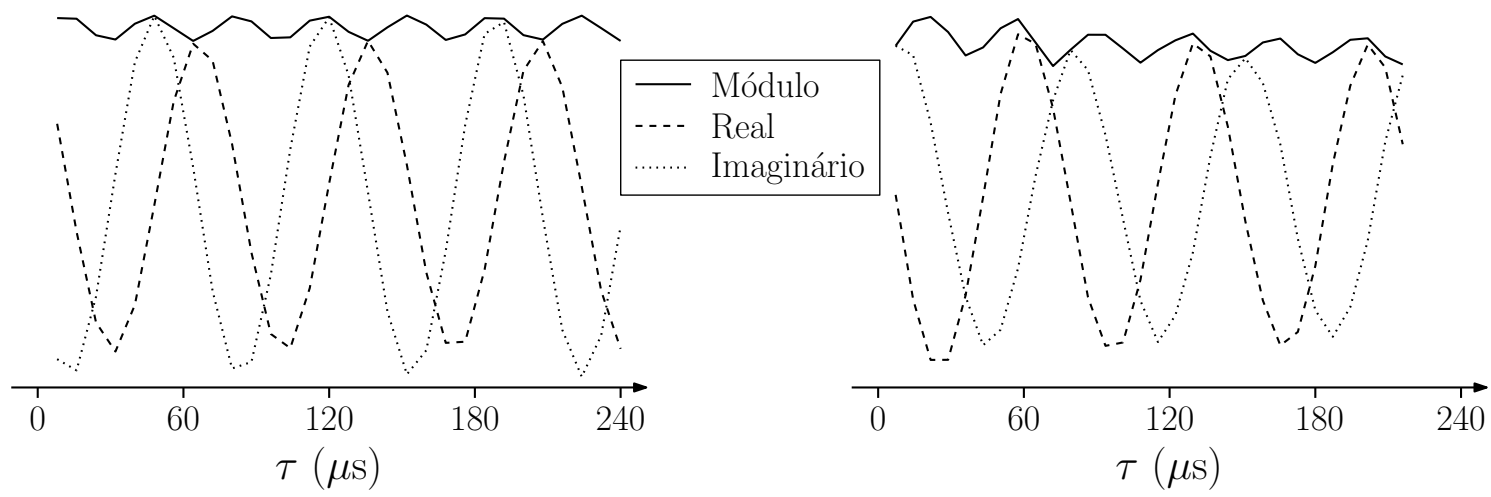

Figura 26: Efeito de erro de fase na tomografia da coerência $\rho_{13}$ evidenciado pela oscilação do módulo. a) Simulação do experimento de tomografia utilizando-se um tempo de espera de $5 \mu$ s entre o final do pulso não-seletivo e o começo da aquisição. b) Tomografia experimental sem correção de fase. Os parâmetros da hamiltoniana em ambos os casos são aproximadamente iguais.

apresentam oscilação quadrupolar. Observa-se que ocorre uma oscilação mínima no módulo dessas coerências, indicando um processo de seleção de coerências bastante eficiente. Além disso, dos ajustes senoidais, percebe-se que o resultado está bastante consistente com o esperado pela matriz w. A figura 28 apresenta os resultados experimentais da dinâmica dos elementos que, de acordo com w, não possuem oscilação quadrupolar. Para os elementos diagonais foi utilizado um intervalo de amostragem temporal bem mais longo que nos outros elementos. Nesse caso, efeitos de relaxação tornam-se relevantes e a dinâmica não é descrita mais pela hamiltoniana quadrupolar. A tomografia de todos os elementos das Figuras 27 e 28 seguiu o procedimento da seção anterior utilizando os ângulos ótimos de nutação.

Para realizar o segundo experimento desta seção foram otimizados SMP's para a criação de estados proporcionais aos tensores de polarização $\mathrm{T}_{l m}$. Assim, considerando somente as componentes de ordem não negativa, foi necessário criar 9 estados iniciais. A tomografia experimental desses estados, bem como uma comparação com os estados teóricos encontram-se expostos nas Figuras 29 e 30. É importante notar que devido à hermiticidade de $\rho$, os estados criados são obrigatoriamente proporcionais à soma do operador escolhido com o seu adjunto, ou seja, $\rho \propto \mathrm{T}_{l m}+\mathrm{T}_{l m}^{\dagger}=\mathrm{T}_{l m}+(-1)^{m} \mathrm{~T}_{l,-m}$. Nas figuras, juntamente com a tomografia de cada estado, encontram-se duas medidas de fidelidade entre o estado experimental e o teórico. Ambas as medidas correspondem à definição (2.86). A fidelidade $F$ refere-se à comparação entre os dois estados completos, enquanto que $F^{\prime}$ leva em conta somente os elementos de $\rho$ experimental que possuem o valor da coerência $m$ escolhida. 


$\nabla$ Módulo $\square$ Real $\bigcirc$ Imaginário $\_$Ajuste senoidal
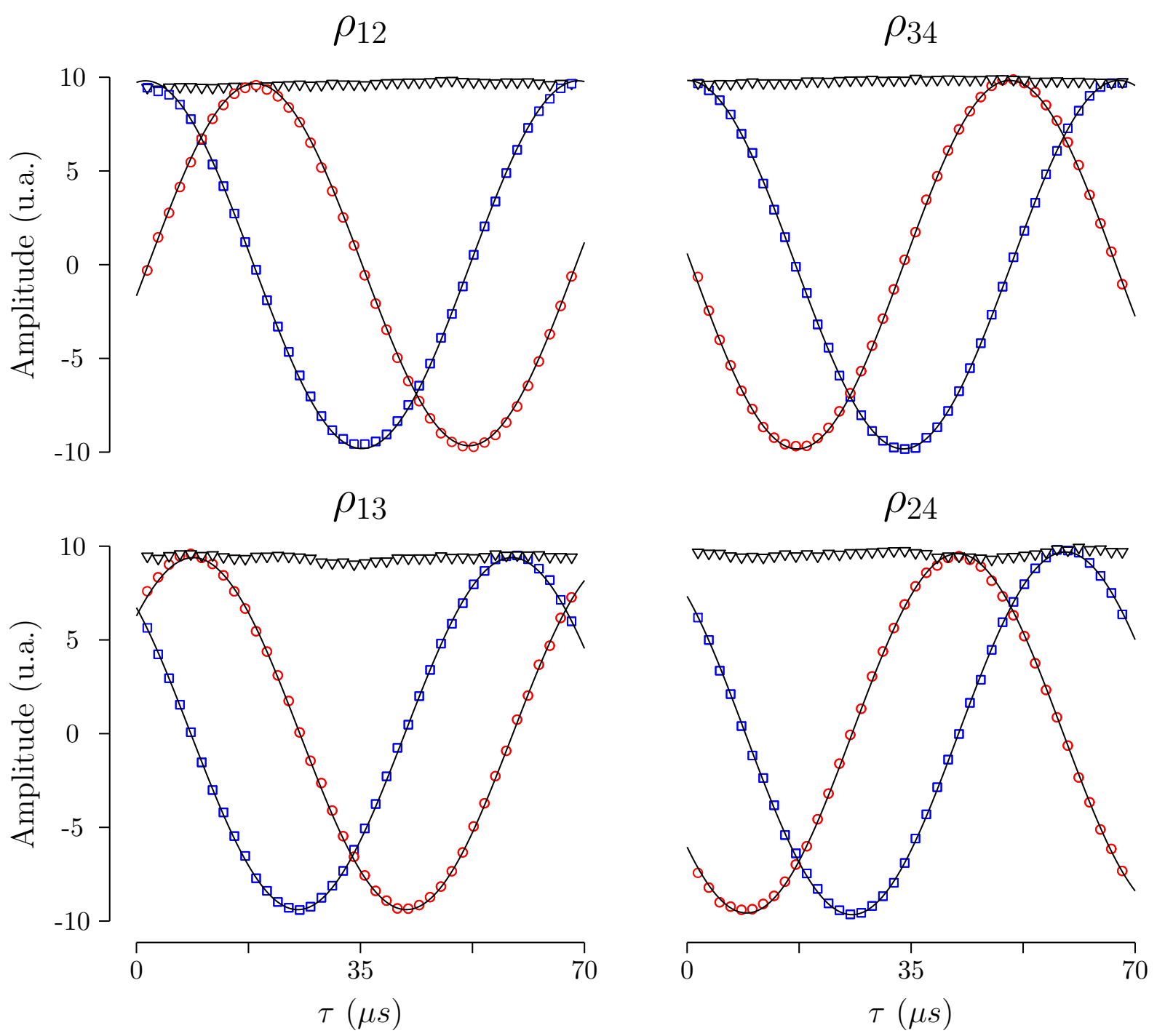

Figura 27: Resultado experimental da dinâmica dos elementos da matriz densidade que oscilam com a freqüência quadrupolar $\omega_{Q}$. Para os elementos $\rho_{12}$ e $\rho_{13}$ observa-se a fase cossenoidal da parte real adiantada com relação à parte imaginária, enquanto que para os elementos $\rho_{34} \mathrm{e}$ $\rho_{24}$ ocorre o oposto. Esse resultado está em pleno acordo com o esperado pela matriz w. O valor médio obtido para as frequiências quadrupolares via ajuste senoidal foi $\omega_{Q}=15,0 \pm 0,1$ $\mathrm{kHz}$. O valor médio obtido para a fase relativa entre as componentes real e imaginária foi $\Delta \phi=90^{\circ} \pm 2^{\circ}$. 

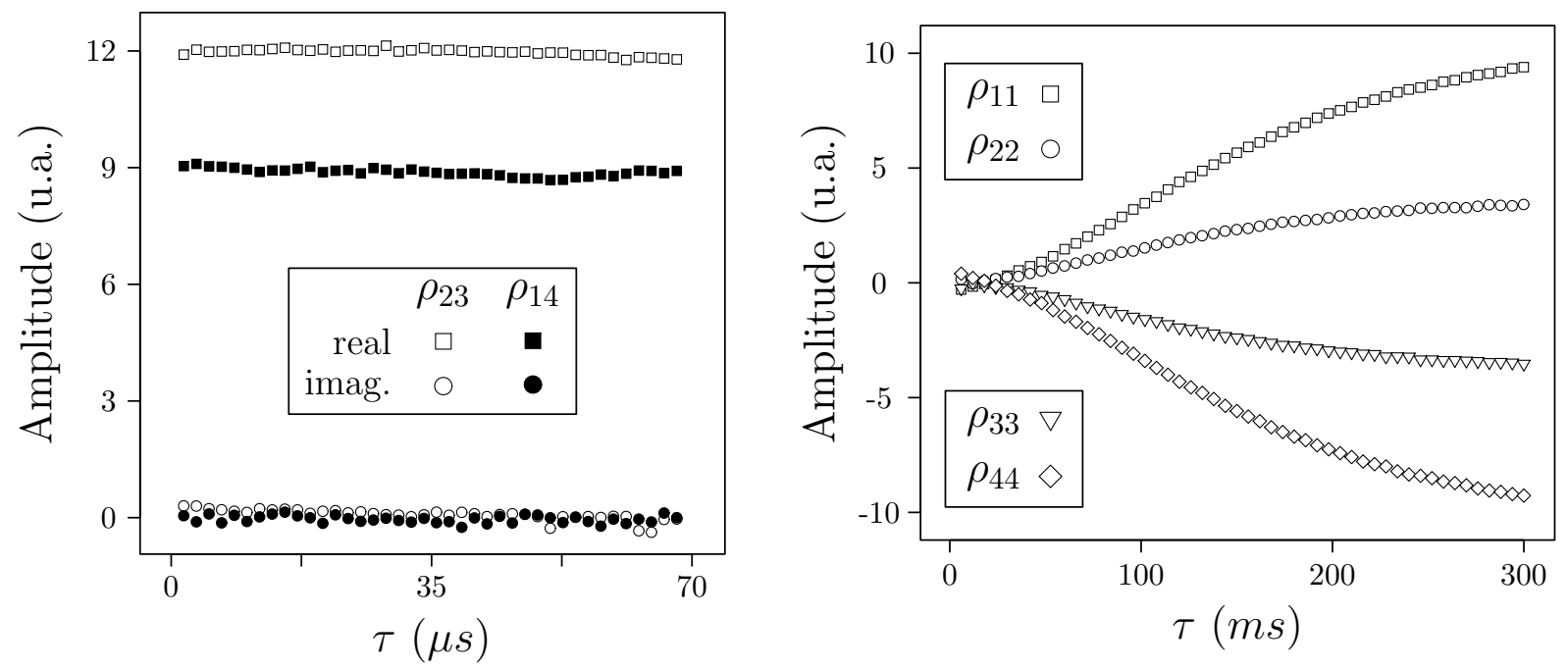

Figura 28: Resultado experimental da dinâmica dos elementos da matriz densidade que não oscilam com a frequêencia quadrupolar $\omega_{Q}$. Os elementos $\rho_{23}$ e $\rho_{14}$ mantêm-se aproximadamente constantes conforme previsto por w. A evolução dos elementos diagonais corresponde ao processo de retorno ao estado de equilíbrio térmico devido a efeitos de relaxação. Nesse caso a dinâmica não é mais descrita pela hamiltoniana quadrupolar, pois o tempo de evolução $\tau$ é suficientemente grande para que interações externas não possam ser desprezadas.

A expressão para as amplitudes espectrais dada pela Equação (3.46):

$$
\bar{S}_{i, i+1}\left(m^{\prime}\right)=\sum_{l}\left(a_{l m^{\prime}}\right)^{*} d_{1, m^{\prime}}^{l}(-\theta)\left[A_{l}\right]_{i, i+1}
$$

reduz-se a:

$$
\bar{S}_{i, i+1}\left(m^{\prime}\right)=\left(a_{l^{\prime} m^{\prime}}\right)^{*} d_{1, m^{\prime}}^{l^{\prime}}(-\theta)\left[A_{l^{\prime}}\right]_{i, i+1}
$$

para os estados $\rho$ em que as componentes de ordem $m^{\prime}$ só possuem o rank $l^{\prime}$ diferente de zero. Dessa forma, pode-se escolher uma das amplitudes do espectro e observar a variação da intensidade com o ângulo de nutação $\theta$. Como essa dependência é proporcional à respectiva função de Wigner $d_{1, m^{\prime}}^{l^{\prime}}$, esse tipo de experimento torna-se útil para validar o emprego da tomografia com ângulos ótimos, a qual é baseada justamente na forma das funções de Wigner. A Figura 31 apresenta os resultados experimentais obtidos juntamente com a comparação com as curvas teóricas de $d_{1, m^{\prime}}^{l^{\prime}}(-\theta)$. Vale notar que esses resultados foram obtidos sem nenhum processo de reconstrução, ou seja, eles correspondem às amplitudes espectrais diretamente obtidas dos espectros após a aplicação da seqüência de pulsos de geração de pseudo-puros e tomografia. Em especial, os estados $\mathrm{T}_{1,1}+\mathrm{T}_{1,1}^{\dagger}$ e $\mathrm{T}_{1,0}$ foram obtidos sem o uso de SMP's, pois o primeiro é proporcional ao estado $\mathrm{I}_{x}$, sendo trivialmente produzido com um pulso de excitação, e o segundo é proporcional ao estado de equilíbrio $\mathrm{I}_{z}$. Dos gráficos da Figura 31 observa-se uma ótima concordância com as curvas teóricas. Em especial, percebe-se que as regiões de máximo das curvas são bem descritas pelos pontos experimentais. 

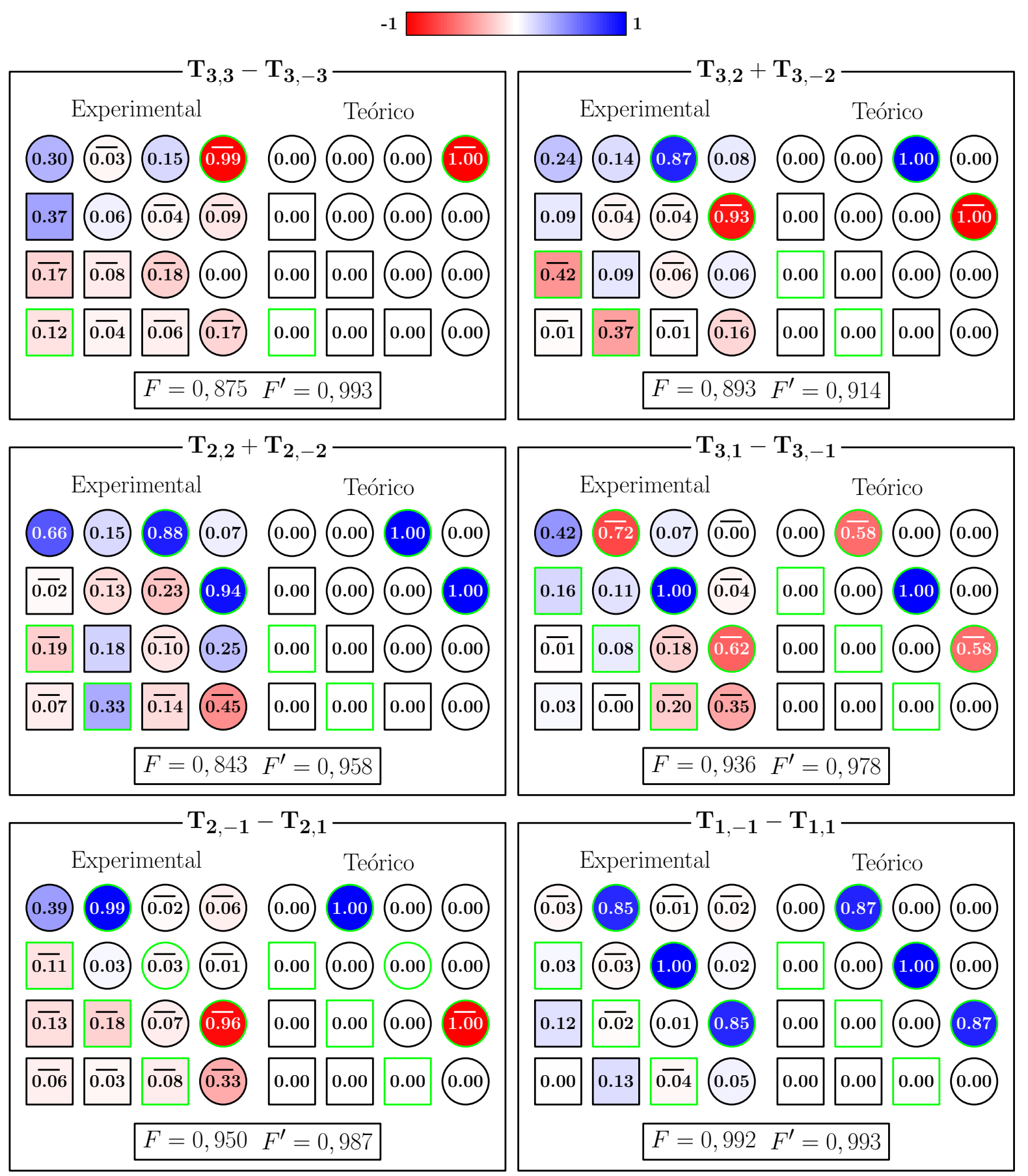

Figura 29: Estados proporcionais à base de operadores irredutíveis de polarização $\mathrm{T}_{l m}+$ $(-1)^{m} \mathrm{~T}_{l,-m}$. A variável $F$ refere-se à fidelidade entre os estados experimental e teórico completos. A variável $F^{\prime}$ refere-se à mesma fidelidade considerando-se apenas os elementos que contribuem para a ordem de coerência em questão. Na figura, as molduras que contêm esses elementos estão representadas com bordas claras. 


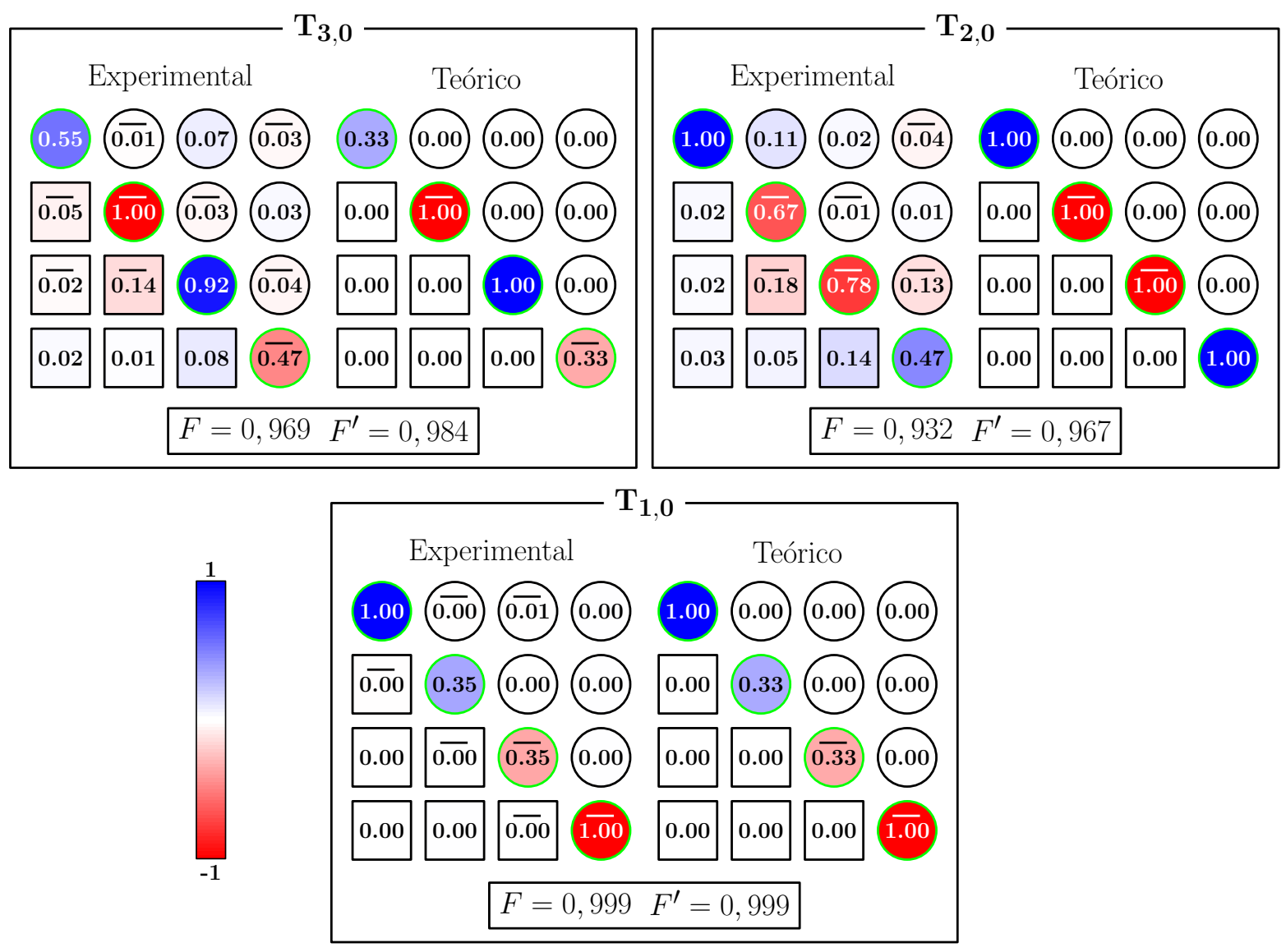

Figura 30: Continuação dos resultados da Figura 29 contendo os operadores de ordem 0.

O único desvio mais intenso ocorre para os ângulos próximos de $\pi / 2$ no caso da função $d_{1,0}^{3}$ na parte inferior direita da Figura 31. Nesse caso foi possível constatar um interessante efeito de mistura com outros ranks. Com uma fidelidade $F^{\prime}$ de 0,993, vide Figura 30, seria de se esperar uma contribuição desprezível de outros ranks além de $l=3$. No entanto, encontra-se para o estado tomografado $T_{3,0}$ as fidelidades 0,166 e 0,053 com relação aos estados teóricos $T_{1,0}$ e $T_{2,0}$, respectivamente. Aliando-se esse resultado ao fato de que a função de Wigner $d_{1,0}^{1}$ é intensa para ângulos próximos de $\pi / 2$, ocorre uma contribuição não desprezível dessa componente para a curva de nutação. Levando em conta esse efeito obtém-se a curva tracejada no gráfico de $d_{1,0}^{3}$, a qual concorda bastante bem com os pontos experimentais. Vale notar que devido aos termos $\left[A_{l^{\prime}}\right]_{i, i+1}$ serem nulos no caso da linha central $(i=2)$ das componentes de rank $l^{\prime}=2$, foram utilizadas as linhas laterais para produzir os gráficos desse rank. No caso das demais componentes utilizou-se a linha central. Uma outra informação importante oferecida pelos resultados experimentais da Figura 31 diz respeito à não-seletividade dos pulsos de leitura da tomografia. Como os resultados experimentais, principalmente na região dos máximos, concordam bastante bem com as funções de Wigner é possível inferir que não houve evolução quadrupolar significativa durante os pulsos de tomografia, justificando portanto o uso de pulsos de leitura retangulares ao 
Amplitudes espectrais versus Ângulo de nutação
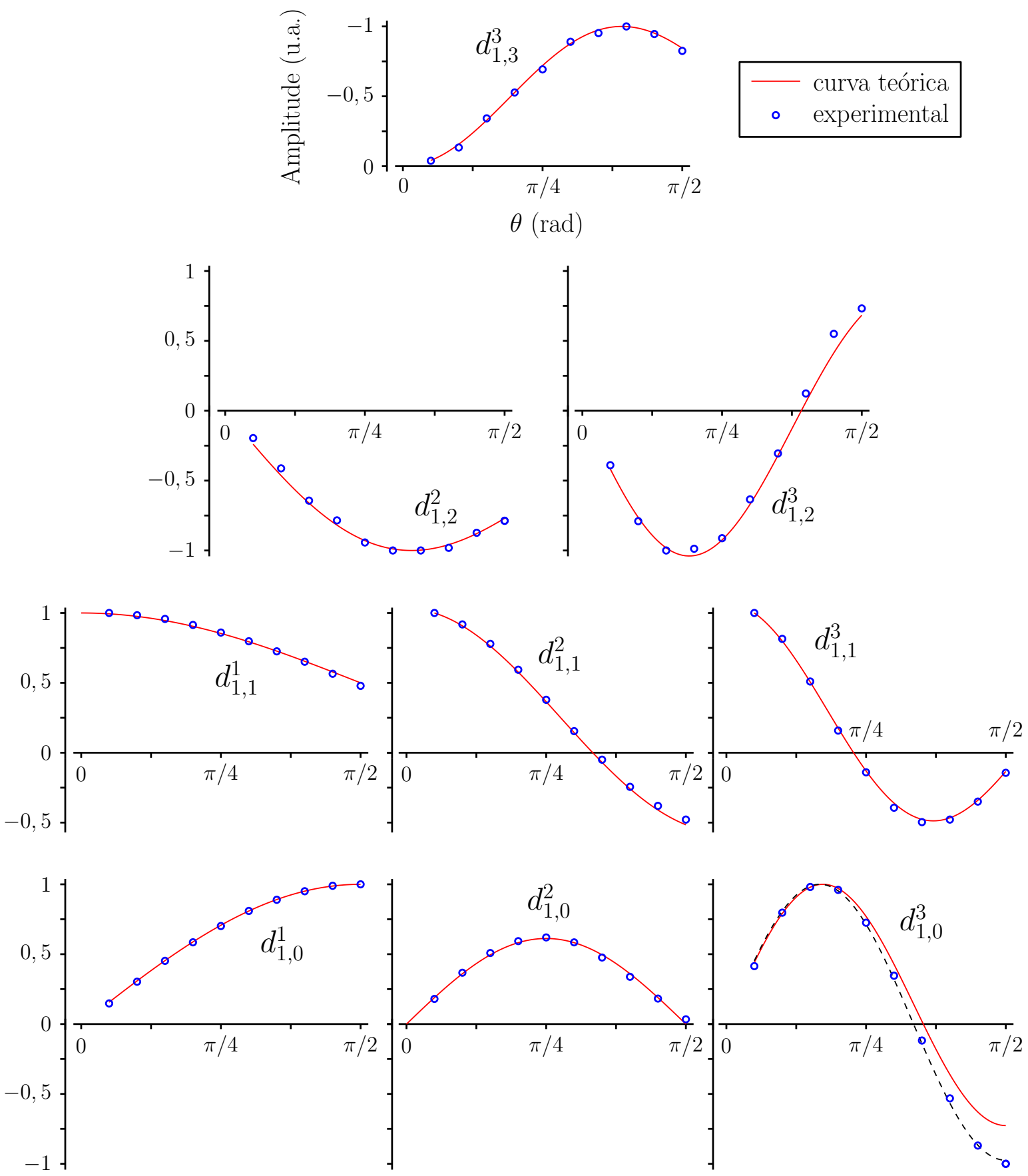

Figura 31: Variação das amplitudes espectrais com o ângulo de nutação para os estados das Figuras $29 \mathrm{e} 30$. Cada estado $\mathrm{T}_{l m}+\mathrm{T}_{l m}^{\dagger}$ produz amplitudes espectrais que variam com o ângulo de nutação conforme as funções $d_{1, m}^{l}(-\theta)$. A legenda e os eixos do gráfico no topo da figura também se aplicam aos demais gráficos. As amplitudes dos estados de rank $l=0,1,3$ correspondem à medida da linha central, enquanto que os estados de rank $l=2$ correspondem a uma das linhas laterais. A curva tracejada no gráfico da função $d_{1,0}^{3}$ leva em conta a componente $d_{1,0}^{1}$ que contribui de forma substancial para a curva de nutação da coerência de ordem 0 do estado $T_{3,0}$ criado experimentalmente. 


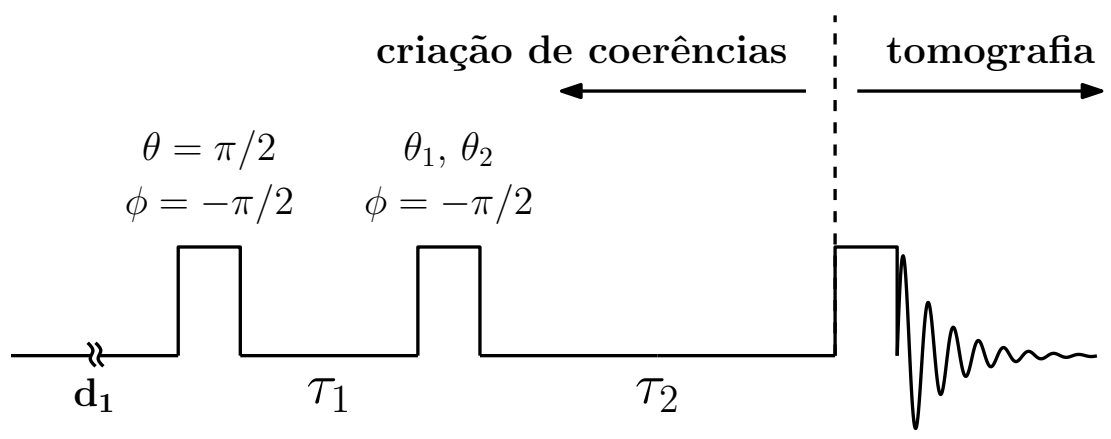

Figura 32: Sequiência de pulsos simples utilizada para a criação de estados com coerências de ordens 2 e 3. Nesse diagrama subentende-se que as fases $\beta_{n}$ e $\phi_{n}^{\prime}$ estão sendo aplicadas para os pulsos de criação de coerências e tomografia, respectivamente.

invés de pulsos modulados de auto-refocalização discutidos na seção 2.2 .

Portanto, dos resultados obtidos a partir dos dois experimentos acima é possível verificar a eficiência do procedimento de seleção de coerências que resultou nos gráficos das Figuras 27]e 28. A concordância entre a descrição analítica das rotações exposta na seção 3.2 e os resultados experimentais da Figura 31 reforça a eficiência do método de tomografia proposto.

A terceira e última série de experimentos exposta nesta seção tem como objetivo fazer uma validação quantitativa mais precisa do método de TEQ. Conforme discutido anteriormente, torna-se difícil discriminar a contribuição do método de TEQ para o resultado final de um algoritmo quântico usando-se SMP's. Isso porque desvios nos resultados esperados podem ser devidos à imperfeições nas formas dos pulsos modulados. Uma maneira de contornar esse problema é tomografando estados criados por sequiências de pulsos simples que em princípio não possuem relação com operações de CQ. Utilizando-se tais seqüências obtém-se mais confiança na matriz densidade esperada, colocando o método de TEQ mais exclusivamente à prova. A idéia portanto é aplicar seqüências que criem coerências de diferentes ordens para que a tomografia possa ser testada sobre todos os elementos da matriz densidade.

O experimento de tomografia de coerências de ordem 0 mais simples que pode ser realizado é a tomografia do estado de equilíbrio térmico, o qual já foi realizado e corresponde ao operador $\mathrm{T}_{1,0}$ na Figura 30. Nele foi possível encontrar uma fidelidade praticamente ideal: $F=0,999$. Um teste de tomografia de coerências de ordem 1 também já foi apresentado na Figura 29. Nesse caso, um estado proporcional a $\mathrm{I}_{x}$ foi tomografado, resultando em uma fidelidade $F=$ 0,992. Para a criação de coerências de ordens mais altas é necessário, além do uso de pulsos de rotação, períodos de evolução livre sob a hamiltoniana quadrupolar. Isso porque a aplicação de operações de rotação sobre o estado $\mathrm{I}_{z}$ só produz projeções do operador momento angular $\mathbf{I}$, as quais possuem apenas coerências de ordem 0 e 1. 
a) Curva de transferência de rank

b) Curva de transferência de ordem

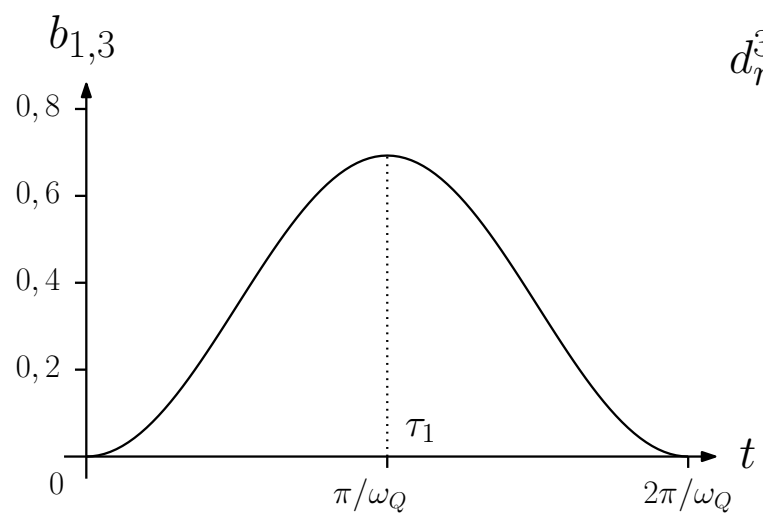

$d_{m, 1}^{3}-d_{m,-1}^{3}$

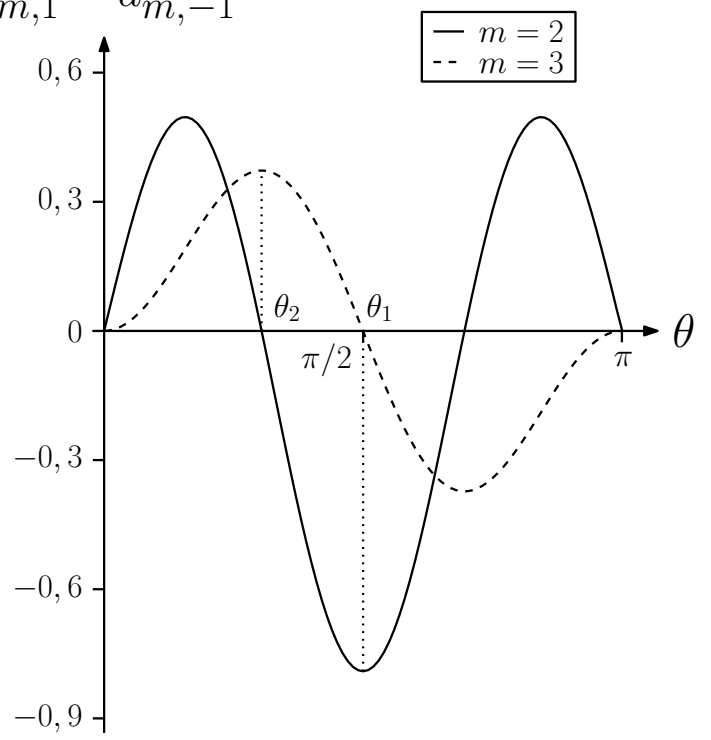

Figura 33: a) Evolução da componente de rank 1 e ordem 1 para a componente de rank 3 e ordem 1 sob a hamiltoniana quadrupolar. b) Evolução da componente de rank 3 e ordem 1 para as componentes de rank 3 e ordens 2 e 3 sob o pulso de rotação. As linhas pontilhadas verticais indicam os valores dos parâmetros $\tau_{1}, \theta_{1}$ e $\theta_{2}$ usados na sequiência da Figura 32 .

Conforme discutido na Equação (3.52), devido ao fato da hamiltoniana livre ser diagonal na base utilizada, evoluções livres preservam a ordem e alteram somente o rank dos operadores irredutíveis. Assim, utilizando-se exclusivamente pulsos não-seletivos e evoluções livres, a seqüência mais simples para a criação de estados com $m>1$ é através da seqüência da Figura 32. Nela constam dois pulsos de rotação intercalados por dois períodos de evolução. O primeiro pulso é responsável pela criação das coerências de ordem 1 e rank 1 , as quais evoluem sob $\mathrm{H}_{Q}$. Assim, no período de evolução livre, componentes de ranks mais altos são produzidas. Dessa forma, a aplicação subseqüente de um pulso de rotação pode produzir coerências de ordens tão altas quanto o valor dos ranks, vide Equação (3.27). O segundo período de evolução livre não é de fato obrigatório, no entanto ele é utilizado para que o pulso de tomografia não seja adjacente ao segundo pulso, causando imperfeições devido aos delays internos do hardware discutidos na seção anterior.

Portanto, conforme mostrado na Figura 32, o primeiro pulso de excitação possui fase de $-\pi / 2$ e aplica uma rotação de $\pi / 2$ na magnetização. Dessa forma, é criado um estado de ordem 1 com a máxima amplitude. A etapa seguinte de evolução livre deve produzir componentes de rank 2 ou 3 também com a máxima amplitude possível. Para descobrir qual é o tempo $\tau_{1}$ que produz essa maximização é útil calcular o coeficiente $b_{l, l^{\prime}}$ definido na Equação (3.52) e calculado analogamente à Equação (3.57). Esse coeficiente corresponde à amplitude do opera- 
dor de rank $l^{\prime}$ produzido após a evolução livre do operador de rank $l$. É possível mostrar que $b_{1,2}$ é identicamente nulo. No entanto, $b_{1,3}$ não é nulo e a sua dependência com o tempo de evolução está exposta no gráfico da Figura 33 a). Observa-se que a máxima transferência de rank corresponde à metade do período quadrupolar. Dessa forma, a componente $T_{3,1}-T_{3,-1}$ ao ser rodada pela ação do segundo pulso pode produzir coerências de até $3^{a}$ ordem. O gráfico da Figura 33 b) contém a dependência angular das funções $d_{m, 1}^{3}-d_{m,-1}^{3}$ que determinam as amplitudes dos operadores criados com o segundo pulso de rotação, vide Equação (3.27). Os ângulos $\theta_{1} \approx 1,57$ rad e $\theta_{2} \approx 0,96$ rad foram escolhidos pois maximizam a amplitude das componentes de ordem $m=2$ e $m=3$, respectivamente. A fase utilizada para esses pulsos foi $\phi=-\pi / 2$. O segundo período de evolução livre foi escolhido de forma tal que o estado $\rho$ sofresse uma evolução quadrupolar completa, $\tau_{2}=2 \pi / \omega_{Q}$, não causando portanto modificação no estado obtido após a aplicação do segundo pulso. Os resultados experimentais das tomografias juntamente com os estados teóricos esperados encontram-se na Figura 34. Nesse experimento também foram calculadas duas fidelidades diferentes para cada estado. A fidelidade $F$ é calculada entre o estado experimental tomografado e o estado teórico, assumindo que os dois pulsos da Figura 32 produzem rotações ideais sobre o spin. No entanto, devido ao tempo finito do pulso de rotação, ocorre uma pequena evolução quadrupolar sobre o estado do sistema. As matrizes densidade teóricas da Figura 34 foram calculadas levando em conta essa evolução, sendo que a fidelidade $F^{\prime}$ é calculada entre as matrizes experimentais e essas matrizes teóricas. Além dos altos valores de fidelidade obtidos, $F^{\prime}=0,987$ e $F^{\prime}=0,990$, foi possível verificar o comportamento previsto pelo gráfico da Figura 33, em que para os ângulos $\theta_{1}$ e $\theta_{2}$ ocorre a criação alternada das coerências de $2^{a}$ e $3^{a}$ ordens. Abaixo das matrizes de cada experimento encontram-se os espectros experimentais medidos utilizando-se o procedimento experimental de tomografia descrito na seção anterior. Foram as amplitudes desses espectros que deram origem às respectivas matrizes experimentais após a etapa de reconstrução. Percebe-se desses espectros que a forma das linhas espectrais e a fase dos elementos correspondentes estão diretamente relacionadas, ou seja, linhas absortivas e dispersivas dão origem a elementos reais e imaginários, respectivamente. 


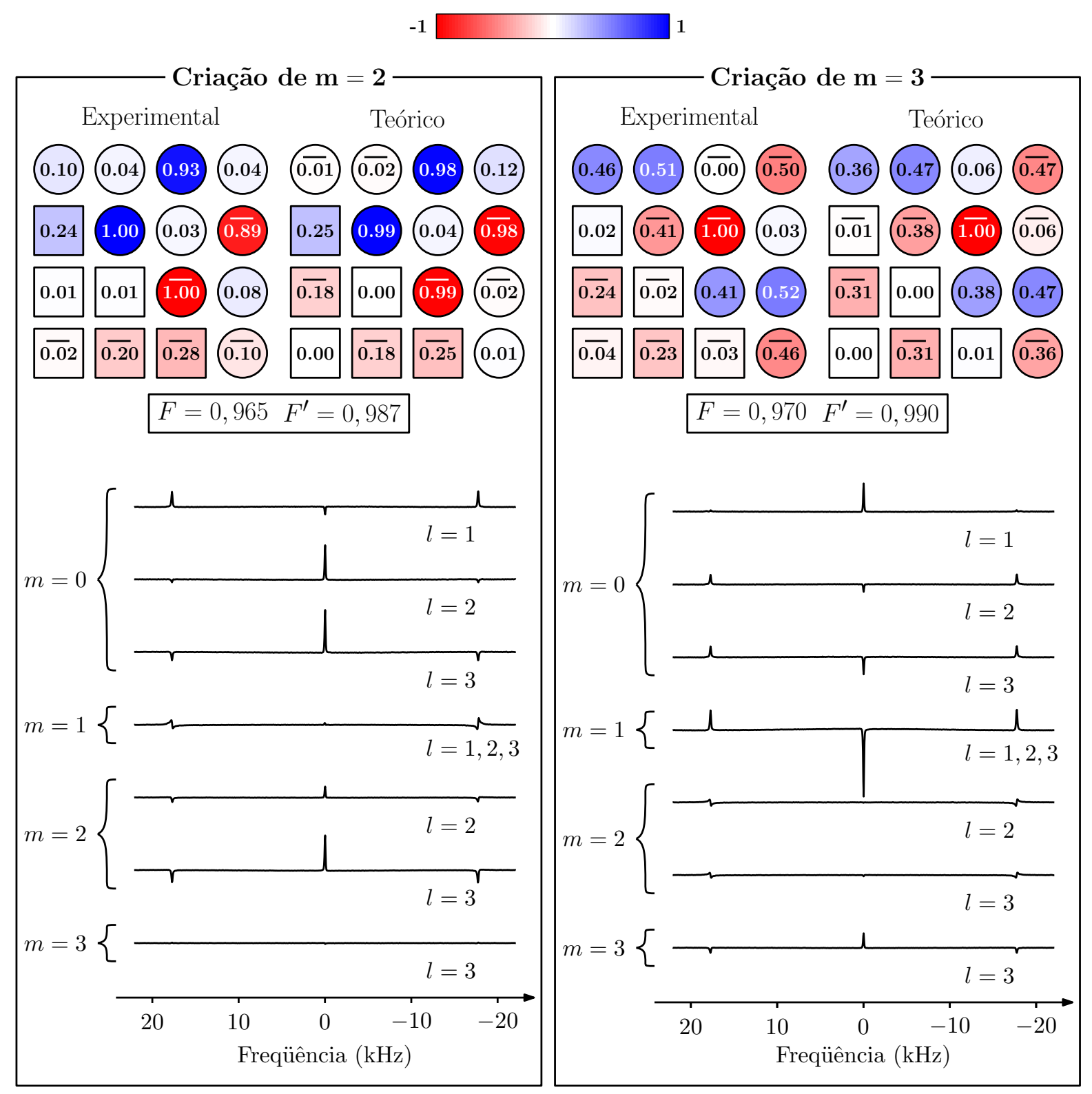

Figura 34: Resultado da tomografia dos experimentos de criação de coerências de $2^{a}$ e $3^{a}$ ordens, vide Figura 32. Os estados teóricos desta figura levam em conta a evolução quadrupolar durante os pulsos de rotação, resultando na fidelidade $F^{\prime}$ com os estados experimentais. A variável $F$ refere-se à fidelidade considerando-se estados teóricos obtidos com pulsos de rotação ideais. Abaixo das matrizes densidade estão os respectivos espectros medidos que deram origem às matrizes experimentais. O índice $m$ refere-se à ordem de coerência selecionada e $l$ refere-se ao rank maximizado em cada seleção. 


\subsection{Aplicações do método de TEQ}

Esta seção contém as aplicações da otimização de SMP's e do método de tomografia para a realização de operações de computação quântica na amostra de cristal líquido de DSS. Foram implementados o algoritmo de Deutsch e o algoritmo de busca de Grover para 2 q-bits. Também foram realizadas experimentalmente as transformações dos estados da base computacional para a base de Bell. Ainda com a amostra de DSS, realizou-se a tomografia do processo de relaxação de vários estados pseudo-puros.

\subsubsection{Criação dos Estados de Bell}

A base de estados de Bell possui características interessantes principalmente por suas propriedades de emaranhamento. Estados de Bell são normalmente a base dos algoritmos de criptografia, além de aparecerem com freqüência nos demais algoritmos quânticos. Por esses motivos, torna-se interessante implementá-los experimentalmente e verificar a eficiência do processo via o método de Tomografia exposto neste trabalho.

A Figura 35 contém o circuito utilizado para a criação dos estados de Bell a partir dos estados da base computacional. Aplicando-se os estados $|00\rangle,|01\rangle,|10\rangle$ e $|11\rangle$ na entrada do circuito, obtêm-se como saída, respectivamente, os estados: $|00\rangle+|11\rangle,|01\rangle+|10\rangle,|00\rangle-|11\rangle$ e $|01\rangle-|10\rangle$. A porta Hadamard, HD, gera a transformação: $|0\rangle \rightarrow|0\rangle+|1\rangle$ e $|1\rangle \rightarrow|0\rangle-|1\rangle$. Note que HD é uma operação genuinamente quântica, pois a sua ação sobre um estado clássico gera um estado superposto. Em todas as transformações expostas nesta seção de aplicações são ignorados os fatores de normalização dos estados. A porta NOT-controlada, $\mathrm{CN}_{A}$, gera a negação do q-bit $B$ somente se o q-bit $A$ for 1 .

A TEQ foi realizada ao final de cada passo do circuito da Figura 35, ou seja, após a criação do estado pseudo-puro, após a aplicação da porta Hadamard e após a aplicação da porta NOTcontrolada. Todos os estados e portas lógicas foram implementados utilizando-se SMP's. As fidelidades numéricas obtidas para os pseudo-puros foram todas superiores a 0,999. As fidelidades e os erros obtidos numericamente para as portas lógicas estão na tabela 7 . Os resultados do experimento encontram-se na Figura 36 . Nessa figura, estão expostas somente as componentes reais de cada elemento de $\rho$. Para esse caso, as matrizes teóricas são todas reais, sendo que as componentes imaginárias nas matrizes medidas são devidas aos erros experimentais.

As matrizes experimentais completas, escritas na notação da Figura 24, encontram-se no Apêndice A.7. A tabela 8 contém as medidas experimentais de fidelidade para todos os estados tomografados nesse experimento. 


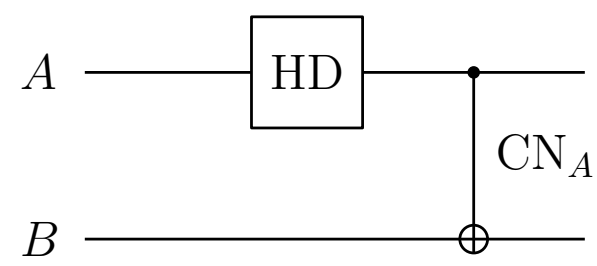

Figura 35: Circuito quântico usado para criar a base de estados de Bell.

\begin{tabular}{|c||c|c|c|c|}
\hline Porta & $\langle F\rangle$ & $\sigma_{F}$ & Duração $(\mu \mathrm{s})$ & Segmentos \\
\hline \hline $\mathrm{HD}_{A}$ & 0,989 & 0,005 & 130,12 & 5 \\
\hline $\mathrm{CN}_{A}$ & 0,993 & 0,003 & 162,35 & 5 \\
\hline
\end{tabular}

Tabela 7: Propriedades numéricas das portas lógicas criadas via técnica SMP e utilizadas no experimento de criação dos estados de Bell. $\langle F\rangle$ e $\sigma_{F}$ correspondem, respectivamente, à fidelidade média e ao desvio padrão obtidos empregando-se o procedimento de estimativa de erros exposto no final da seção 2.2. A duração dos SMP's refere-se a um acoplamento quadrupolar $\left(\omega_{Q}=16,5 \mathrm{kHz}\right)$.

\begin{tabular}{|c||c|c|c|c|}
\hline Etapa & $|00\rangle$ & $|01\rangle$ & $|10\rangle$ & $|11\rangle$ \\
\hline \hline pps & 0,993 & 0,990 & 0,988 & 0,982 \\
\hline $\mathrm{HD}_{A} \mathrm{pps}$ & 0,956 & 0,927 & 0,921 & 0,921 \\
\hline $\mathrm{CN}_{A} \mathrm{HD}_{A} \mathrm{pps}$ & 0,841 & 0,793 & 0,832 & 0,799 \\
\hline
\end{tabular}

Tabela 8: Fidelidades experimentais obtidas da TEQ de cada passo do experimento de criação de estados de Bell.

Observando-se os valores de fidelidade na Tabela 8, percebe-se que as fidelidades dos estados pseudo-puros foram razoavelmente altas. Esse resultado é condizente com o alto valor de fidelidade $(>0,999)$ calculado numericamente. Para os estados obtidos após a aplicação das portas lógicas, percebe-se que a porta $\mathrm{CN}_{A}$ apresentou um resultado aquém do esperado numericamente. Esse resultado parece prevalecer mesmo considerando que houve um detrimento dos estados criados pela transformação $\mathrm{HD}_{A}$. Isso, no entanto, não implica necessariamente em uma má performance daquela porta comparativamente à $\mathrm{HD}_{A}$. Isso porque a medida de fidelidade de uma operação quântica deve levar em conta a aplicação da mesma sobre uma base de operadores, seguindo por exemplo a definição 2.87). Note que as portas lógicas foram aplicadas sobre uma base de vetores de estado (base computacional) e não sobre uma base de operadores. Ainda assim, como a base computacional também é uma base para operadores diagonais, a fidelidade média dos estados $\mathrm{HD}_{A}$ pps fornece a fidelidade da porta sobre esses operadores. Outra possibilidade de explicação das baixas fidelidades experimentais para os estados obtidos após a aplicação da porta $\mathrm{CN}_{A}$ é de que a mesma foi aplicada sobre estados de superposição, os quais correspondem a matrizes densidade que possuem coerências não nulas, vide Figura 36. Como essas coerências nesse sistema específico evoluem sob a interação quadrupolar, ou seja, esses estados não são auto-estados da hamiltoniana estacionária, erros nos delays internos 

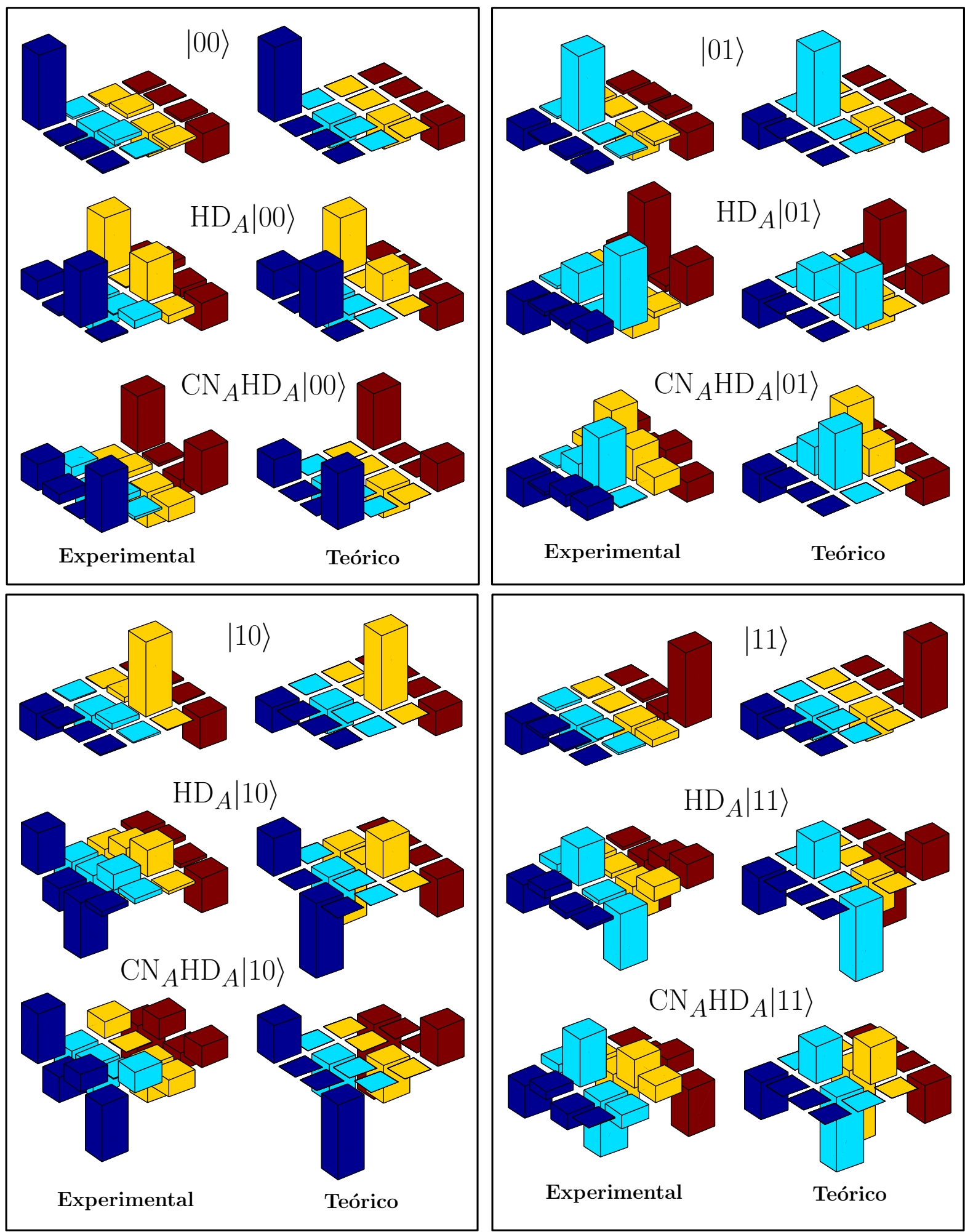

Figura 36: Tomografia das etapas do circuito da Figura 35 para a criação dos estados de Bell. Estão mostrados somente os elementos reais das matrizes densidade. As matrizes experimentais completas encontram-se no Apêndice A.7. 

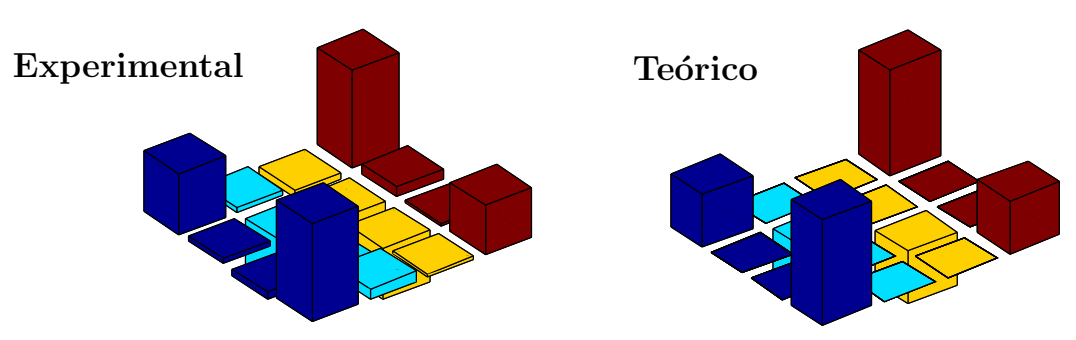

Figura 37: Tomografia do estado do Gato, $|00\rangle+|11\rangle$. Diferentemente do estado $\mathrm{CN}_{A} \mathrm{HD}_{A}|00\rangle$ obtido no experimento da Figura 36, o estado dessa figura foi obtido diretamente do equilíbrio térmico através do procedimento de média temporal.

e nas estimativas desses delays no processo de calibração podem provocar evoluções espúrias do sistema.

Obviamente, os estados pseudo-puros iniciais não precisam corresponder necessariamente à base computacional. Como exemplo de criação de um estado inicial pertencente a uma outra base, a Figura 37 apresenta a tomografia do estado do Gato, $|00\rangle+|11\rangle$. Diferentemente do estado $\mathrm{CN}_{A} \mathrm{HD}_{A}|00\rangle$ mostrado na Figura 36 esse estado foi obtido diretamente sobre o estado de equilíbrio térmico utilizando o procedimento de 4 médias temporais. Devido a isso, obteve-se uma fidelidade de 0,987 que é significativamente superior à fidelidade de 0,841, vide Tabela 8 .

\subsubsection{Algoritmo de Deutsch}

Embora, devido à sua simplicidade, o algoritmo de Deutsch não possua aplicações mais amplas, ele constitui-se em um bom exemplo das idéias subjacentes aos algoritmos genuinamente quânticos. Nesse algoritmo é mostrado claramente como o efeito de interferência quântica permite adquirir a informação global sobre o estado dos q-bits. O seu funcionamento ocorre da seguinte maneira. Considere uma função $f(x)$ para um bit. Só existem quatro possíveis funções desse tipo: $f_{1}(0)=0, f_{1}(1)=0, f_{2}(0)=1, f_{2}(1)=1, f_{3}(0)=0, f_{3}(1)=1$ e $f_{4}(0)=1, f_{4}(1)=0$. Suponha que não se esteja interessado em saber o valor de $f(x)$ para cada possível entrada, mas simplesmente se a função é constante, $f(x)=f(y)$, ou balanceada $f(x) \neq f(y)$ para $x \neq y$. Primeiramente, para que se calcule quanticamente o valor da função $f(x)$ é necessário que a operação seja reversível. No entanto, vê-se claramente que as funções constantes não o são. A maneira de resolver esse problema é usando um segundo q-bit que mantém o registro do estado de entrada de forma a garantir a reversibilidade do processo. Assim, pode-se usar a operação $\mathrm{U}_{f}$ que transforma $|x, y\rangle \rightarrow|x, y \oplus f(x)\rangle$, em que a adição módulo $2, A \oplus B$, corresponde a uma operação NOT-controlada com o controle em $A$. Portanto, fazendo-se $y=0$ obtém-se o valor de $f(x)$ no segundo q-bit. A essência do algoritmo está em aplicar a operação $\mathrm{U}_{f}$ sobre um estado superposto e através de uma mudança de base obter a informação sobre o aspecto constante ou 


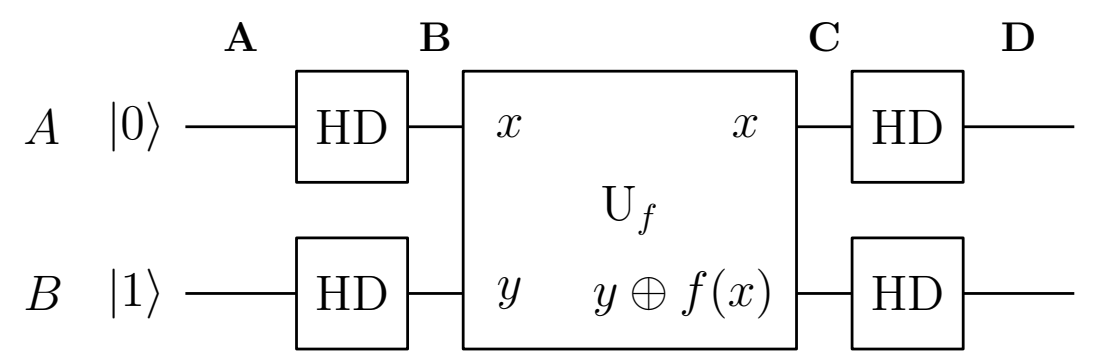

Figura 38: Circuito quântico que implementa o algoritmo de Deutsch.

\begin{tabular}{|c||c|c|c|c|}
\hline Porta $\mathrm{U}_{f}$ & $\langle F\rangle$ & $\sigma_{F}$ & Duração $(\mu \mathrm{s})$ & Segmentos \\
\hline \hline $\mathrm{HD}^{\otimes 2}$ & 0,990 & 0,004 & 152,51 & 5 \\
\hline $\mathrm{NOT}_{B}$ & 0,992 & 0,004 & 139,94 & 5 \\
\hline NOT $_{B} \mathrm{CN}_{A}$ & 0,993 & 0,003 & 165,80 & 5 \\
\hline
\end{tabular}

Tabela 9: Propriedades numéricas das portas lógicas criadas via técnica SMP e utilizadas no experimento de implementação do algoritmo de Deutsch. Todos os símbolos e convenções são os mesmos da Tabela 7 .

balanceado da função. A vantagem com relação a um algoritmo clássico é que, no algoritmo de Deutsch, é necessária a aplicação de $\mathrm{U}_{f}$ apenas uma vez para obter a informação desejada.

O circuito correspondente ao algoritmo está exposto na Figura 38. As portas lógicas que implementam as transformações $\mathrm{U}_{f_{i}}, i=1,2,3,4$, são, respectivamente, a identidade (1), $\mathrm{NOT}_{B}$, $\mathrm{CN}_{A}$ e $\mathrm{NOT}_{B} \mathrm{CN}_{A}$. Pode-se mostrar que essas operações produzem o seguinte estado de saída no circuito da Figura $38 . \pm f(0) \oplus f(1)\rangle|1\rangle$. No experimento implementado neste trabalho, optou-se por utilizar a porta Hadamard de 2 q-bits ao invés da $\mathrm{HD}_{A}$ tradicionalmente usada na última transformação do Algoritmo de Deutsch. $\mathrm{O}$ uso da porta $\mathrm{HD}^{\otimes 2}$ apresenta vantagem com respeito à visualização do resultado obtido, cujas soluções são os estados $\pm|01\rangle$ para funções constantes e $\pm|11\rangle$ para funções balanceadas. As propriedades numéricas das portas lógicas otimizadas via SMP's encontram-se na Tabela 9. A porta $\mathrm{CN}_{A}$ é a mesma do experimento de criação da base de Bell e portanto não foi listada aqui. A operação identidade 1 é obtida diretamente após o passo B, não necessitando da aplicação de pulsos para a sua implementação. A TEQ experimental de cada passo de implementação do algoritmo pode ser visualizada na Figura 39. A ordem temporal de cada passo está representada pelas letras A, B, C e D. No passo $\mathbf{C}$ escolhem-se as funções que produzem os estados constantes: $\mathbf{1}$ e NOT $_{B}$, ou as funções que produzem os estados balanceados: $\mathrm{CN}_{A}$ e $\mathrm{NOT}_{B} \mathrm{CN}_{A}$. As fidelidades experimentais dos estados tomografados em todos os passos do algoritmo encontram-se na Tabela 10 . 


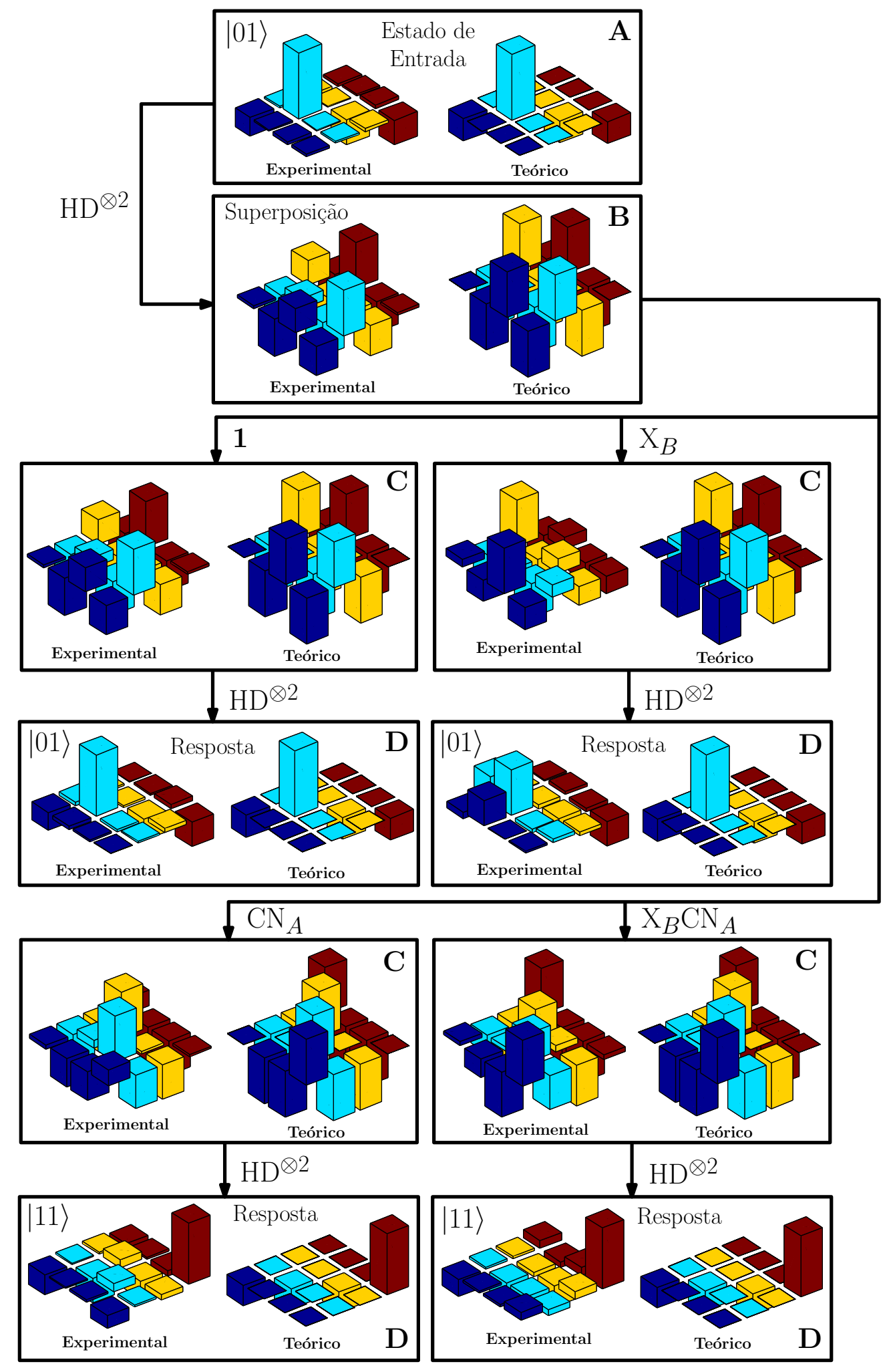

Figura 39: Tomografia das etapas do circuito da Figura 38 para a implementação do algoritmo de Deutsch. A porta $\mathrm{HD}_{A}$ foi substituída pela $\mathrm{HD}^{\otimes 2}$ devido à sua maior facilidade de visualização. Estão mostrados somente os elementos reais das matrizes densidade. As matrizes experimentais completas encontram-se no Apêndice A.7. 


\begin{tabular}{|c||c|c|c|c|c|c|}
\hline Etapa & $|01\rangle$ & $\mathrm{HD}^{\otimes 2}|01\rangle$ & $\mathbf{1}$ & $\mathrm{NOT}_{B}$ & $\mathrm{CN}_{A}$ & $\mathrm{NOT}_{B} \mathrm{CN}_{A}$ \\
\hline \hline A & 0,990 & & & & & \\
\hline B & & 0,901 & & & & \\
\hline C & & & 0,901 & 0,828 & 0,834 & 0,815 \\
\hline D & & & 0,960 & 0,769 & 0,804 & 0,903 \\
\hline
\end{tabular}

Tabela 10: Fidelidades experimentais obtidas da TEQ de cada passo do experimento de implementação do algoritmo de Deutsch.

Dos resultados da Figura 39 e da Tabela 10 , percebe-se que a operação com a porta NOT $_{B}$ é a que apresentou o pior resultado. Entretanto, conforme discutido no experimento de criação dos estados de Bell, uma avaliação mais precisa do desempenho de cada porta só é possível fazendo-se a medida sobre uma base de operadores. Ainda assim, os estados obtidos no passo D são claramente distinguíveis entre si.

É possível associar as matrizes de desvio $\Delta \rho$ em $\mathbf{D}$ a matrizes densidade físicas através da transformação: $\rho=a \Delta \rho+\mathbf{1} / 4$, em que $a$ é escolhida de forma a produzir a matriz $\rho$ com o maior valor de pureza possível, vide Equação 2.6. Nesse caso, medidas projetivas sobre o qbit $A$ forneceriam as probabilidades de acerto, $\rho_{11}+\rho_{22}$, de $95 \%$ e $91 \%$ para as portas 1 e NOT $_{B}$ respectivamente. Para as portas balanceadas $\mathrm{CN}_{A}$ e $\mathrm{NOT}_{B} \mathrm{CN}_{A}$, as probabilidades de acerto, $\rho_{33}+\rho_{44}$, seriam de $93 \%$ e $90 \%$, respectivamente.

\subsubsection{Algoritmo de Grover}

Diferentemente do algoritmo de Deutsch, o algoritmo de busca de Grover apresenta um interesse prático bastante claro. A essência do algoritmo está na operação de oráculo, que permite obter um elemento de uma lista não-ordenada de tamanho $N$ em um tempo da ordem de $\sqrt{N}$. O ganho polinomial no processo de busca representa vantagem sobre a classe de problemas NP-completos [19]. Além disso, o algoritmo pode ser adaptada para um sistema de 2 q-bits, o que permite a sua implementação utilizando-se a amostra de DSS.

A descrição detalhada do funcionamento do algoritmo não será apresentada aqui, podendo ser encontrada em [19]. A exposição aqui presente se limitará à descrição da constituição do circuito quântico correspondente ao caso de 2-qbits, o qual está representado na Figura 40. Na Figura 40.a), o estado de entrada corresponde ao $|00\rangle$. Sobre esse estado, aplica-se a porta Hadamard nos 2 q-bits para gerar o estado de superposição $|\psi\rangle$, que contém todas as combinações dos dois bits com igual peso. A partir daí, o chamado operador de Grover, G, é aplicado repetidamente um número de vezes da ordem de $\sqrt{N}$, até que o estado gerado possua uma alta probabilidade de ser a resposta do problema. No caso geral, pode haver a necessidade 
a)

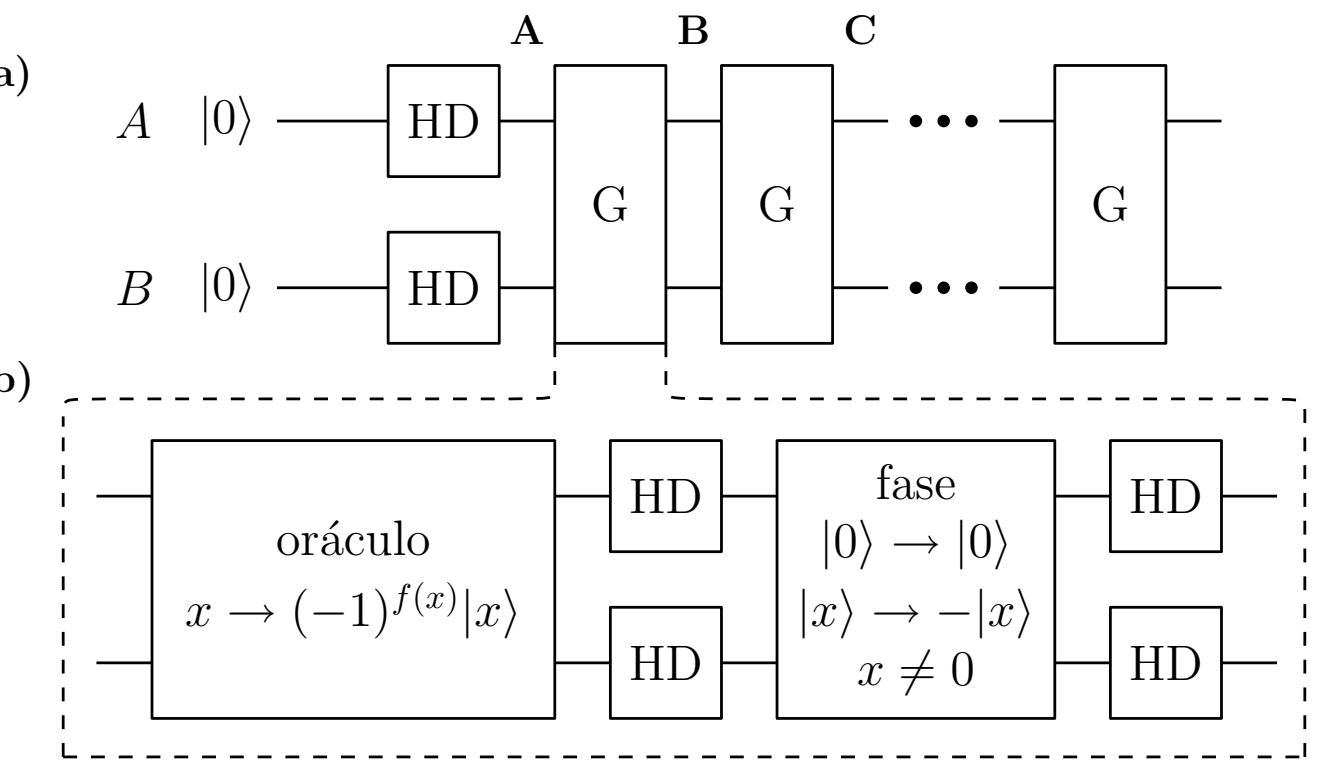

Figura 40: Circuito quântico que implementa o algoritmo de Grover. a) Representação da iteração do operador de Grover. b) Composição do operador G.

do uso de q-bits auxiliares para computar a operação sobre os q-bits da lista de soluções do problema. A Figura 40,b) contém a composição do operador de Grover. Ele é constituído pelo operador oráculo, $\mathrm{O}$, seguido por uma porta de fase inserida entre duas Hadamard de dois q-bits.

A função $f(x)$ no oráculo é que discrimina a solução do problema, fornecendo $f(x)=1$ se $x$ for solução e $f(x)=0$ em caso contrário. Pode-se mostrar que o operador de fase junto com as duas Hadamard corresponde a: $2|\psi\rangle\langle\psi|-1$. Dessa forma, o operador de Grover pode ser escrito como: $\mathrm{G}=(2|\psi\rangle\langle\psi|-\mathbf{1}) \mathrm{O}$.

As funções $f(x)$ utilizadas pelo oráculo e implementadas experimentalmente neste trabalho são dadas por:

$$
f_{y}(x)=\left\{\begin{array}{lll}
0 & \text { para } & x \neq y \\
1 & \text { para } & x=y
\end{array} .\right.
$$

Em que $x$ e $y$ são quaisquer dos 4 estados de dois bits. Portanto, a operação implementada representa simplesmente o processo de busca de uma dentre as 4 funções $f_{y}$. Escrevendo explicitamente o operador de cada oráculo $\mathrm{O}_{00}, \mathrm{O}_{01}, \mathrm{O}_{10}$ e $\mathrm{O}_{11}$, tem-se, respectivamente:

$$
\left[\begin{array}{rrrr}
-1 & 0 & 0 & 0 \\
0 & 1 & 0 & 0 \\
0 & 0 & 1 & 0 \\
0 & 0 & 0 & 1
\end{array}\right],\left[\begin{array}{rrrr}
1 & 0 & 0 & 0 \\
0 & -1 & 0 & 0 \\
0 & 0 & 1 & 0 \\
0 & 0 & 0 & 1
\end{array}\right],\left[\begin{array}{rrrr}
1 & 0 & 0 & 0 \\
0 & 1 & 0 & 0 \\
0 & 0 & -1 & 0 \\
0 & 0 & 0 & 1
\end{array}\right] \text { e }\left[\begin{array}{rrrr}
1 & 0 & 0 & 0 \\
0 & 1 & 0 & 0 \\
0 & 0 & 1 & 0 \\
0 & 0 & 0 & -1
\end{array}\right] \text {. }
$$



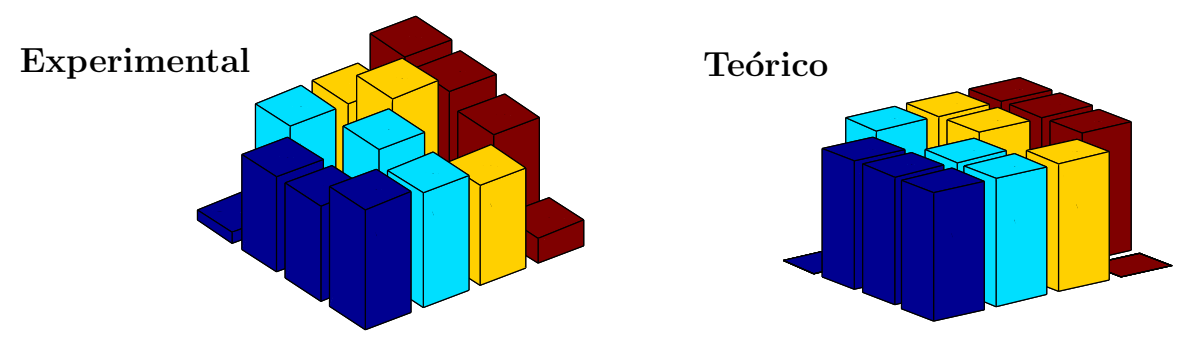

Figura 41: Tomografia do estado inicial de superposição no algoritmo de Grover, $|\psi\rangle=|00\rangle+$ $|01\rangle+|10\rangle+|11\rangle$. Esse estado foi criado diretamente sobre o estado de equilíbrio de forma análoga ao estado do Gato da Figura 37.

\begin{tabular}{|c||c|c|c|c|}
\hline Operação & $\langle F\rangle$ & $\sigma_{F}$ & Duração $(\mu \mathrm{s})$ & Segmentos \\
\hline \hline $\mathrm{G}_{00}$ & 0,988 & 0,006 & 114,50 & 5 \\
\hline $\mathrm{G}_{01}$ & 0,991 & 0,004 & 163,00 & 5 \\
\hline $\mathrm{G}_{10}$ & 0,990 & 0,005 & 141,39 & 5 \\
\hline $\mathrm{G}_{11}$ & 0,984 & 0,008 & 107,30 & 5 \\
\hline
\end{tabular}

Tabela 11: Propriedades numéricas dos operadores de Grover criados via técnica SMP e utilizados no experimento de implementação do algoritmo de busca de Grover. Todos os símbolos e convenções são os mesmos da Tabela 7 .

Os respectivos operadores de Grover multiplicados por 2: $2 \mathrm{G}_{00}, 2 \mathrm{G}_{01}, 2 \mathrm{G}_{10}$ e $2 \mathrm{G}_{11}$, correspondem a:

$\left[\begin{array}{rrrr}1 & 1 & 1 & 1 \\ -1 & -1 & 1 & 1 \\ -1 & 1 & -1 & 1 \\ -1 & 1 & 1 & -1\end{array}\right],\left[\begin{array}{rrrr}-1 & -1 & 1 & 1 \\ 1 & 1 & 1 & 1 \\ 1 & -1 & -1 & 1 \\ 1 & -1 & 1 & -1\end{array}\right],\left[\begin{array}{rrrr}-1 & -1 & 1 & 1 \\ 1 & -1 & -1 & 1 \\ 1 & 1 & 1 & 1 \\ 1 & 1 & -1 & -1\end{array}\right],\left[\begin{array}{rrrr}-1 & 1 & 1 & -1 \\ 1 & -1 & 1 & -1 \\ 1 & 1 & -1 & -1 \\ 1 & 1 & 1 & 1\end{array}\right]$

A implementação experimental do algoritmo foi feita através da otimização de várias portas conjuntas no diagrama da Figura 40. Assim, o estado de superposição $|\psi\rangle$, Figura 41, foi otimizado diretamente através do procedimento de criação de pseudo-puros via SMP's, da mesma forma como foi criado o estado do Gato da Figura 37. A fidelidade experimental obtida foi de 0,878. A operação de Grover também foi implementada através de um único pulso SMP para cada uma das 4 operações. As propriedades numéricas dessas operações encontram-se na Tabela 11. O resultado das tomografias para cinco aplicações seguidas de cada uma das 4 operações G encontra-se nas Figuras 42 e 43. Devido a um equívoco no processo de otimização, os SMP's gerados corresponderam na verdade às operações inversas, $\mathrm{G}^{\dagger}$. Portanto, a ordem temporal correta, caso fosse aplicada a operação $\mathrm{G}$, seria a indicada pelas letras $\mathbf{A}, \mathbf{B}, \mathbf{C}, \mathbf{D}$ e $\mathbf{E}$. 
Em um processo de busca clássica em uma lista desordenada, haveria $25 \%$ de chance de encontrar o elemento almejado na primeira tentativa e um número médio de tentativas necessárias para encontrar o elemento igual a $\sum_{n=1}^{4} n \frac{3^{n-1}}{4^{n}} \approx 1,5$. Pode-se mostrar que com o algoritmo quântico de busca, para o caso de 2 q-bits, o elemento é encontrado sempre na primeira tentativa. Além disso, o algoritmo apresenta um comportamento periódico, em que o número de repetições necessárias para obter uma nova probabilidade máxima de acerto é igual a 3. Esse comportamento é nitidamente observado nos resultados experimentais das Figuras 42 e 43 . As fidelidades experimentais obtidas para as 4 operações $\mathrm{G}$ encontram-se na Tabela 12.

\begin{tabular}{|c||c|c|c|c|}
\hline Iteração & $\mathrm{G}_{00}$ & $\mathrm{G}_{01}$ & $\mathrm{G}_{10}$ & $\mathrm{G}_{11}$ \\
\hline \hline 1 & 0,964 & 0,957 & 0,932 & 0,895 \\
\hline 2 & 0,890 & 0,891 & 0,833 & 0,960 \\
\hline 3 & 0,887 & 0,848 & 0,819 & 0,842 \\
\hline 4 & 0,966 & 0,928 & 0,872 & 0,932 \\
\hline 5 & 0,897 & 0,877 & 0,788 & 0,943 \\
\hline
\end{tabular}

Tabela 12: Fidelidades experimentais obtidas em cada uma das 5 iterações dos operadores de Grover. 


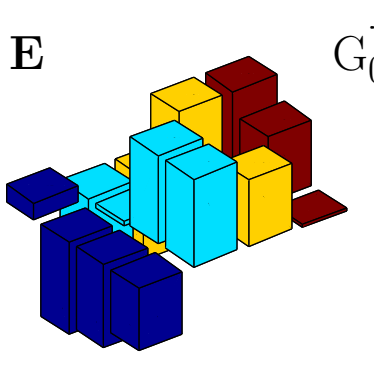

Experimental

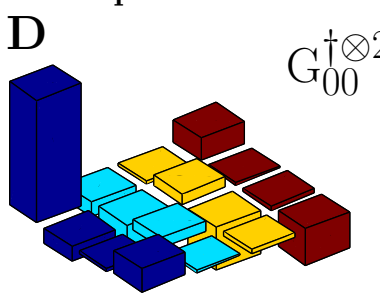

Experimental

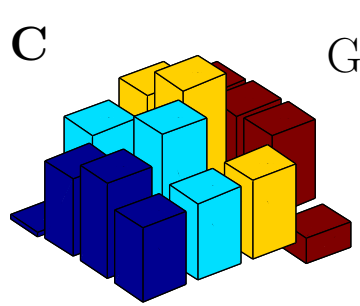

Experimental

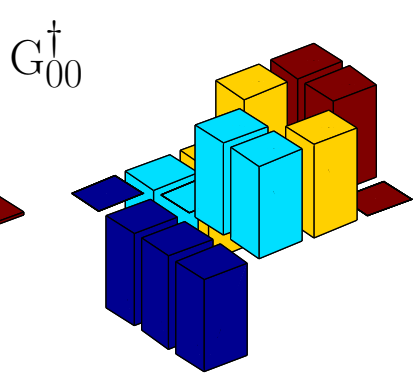

Teórico

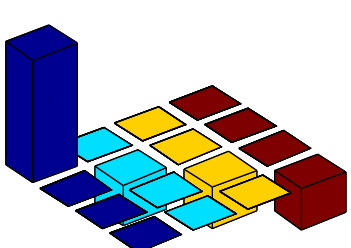

Teórico

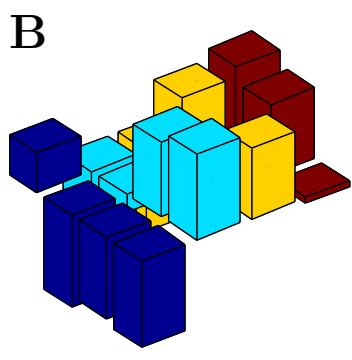

Experimental

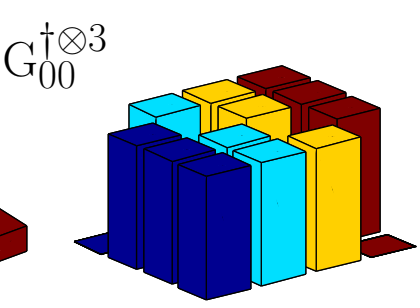

Teórico

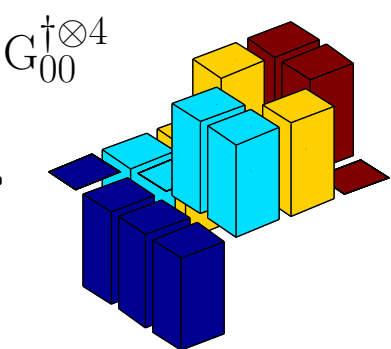

Teórico

\section{A}

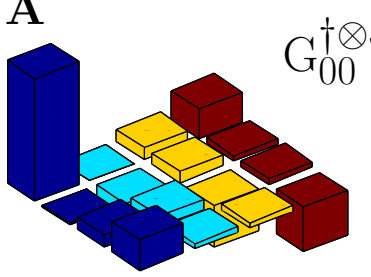

Experimental

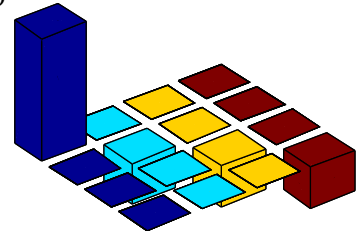

Teórico

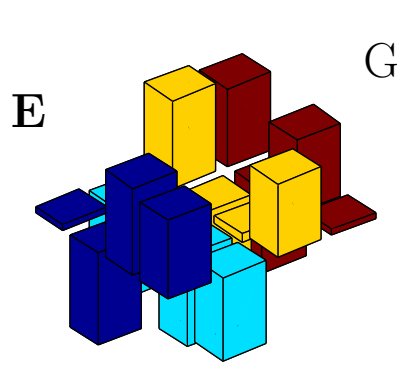

Experimental

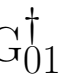

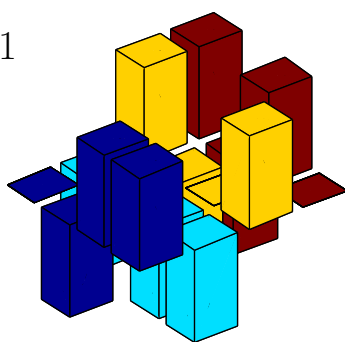

Teórico

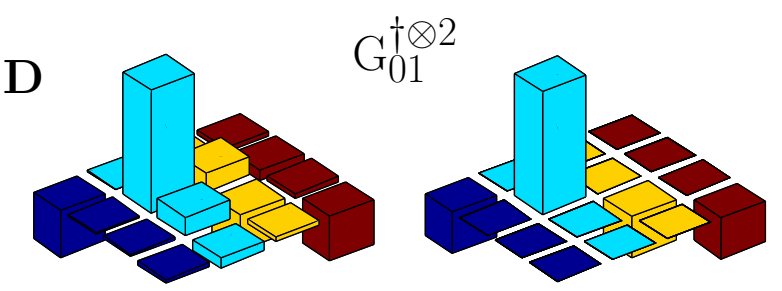

Experimental

Teórico

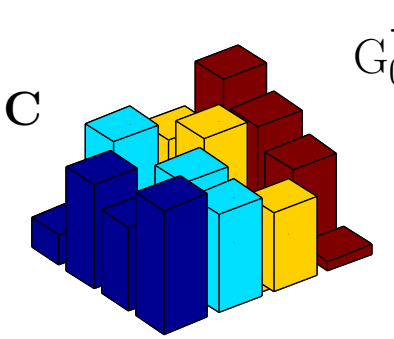

Experimental

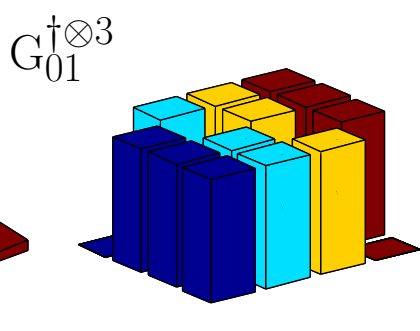

Teórico

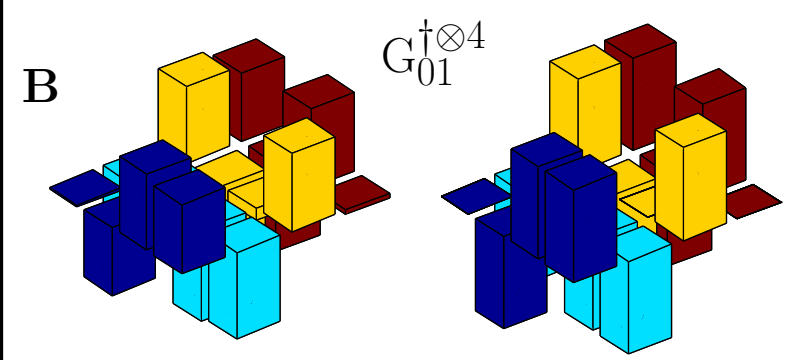

Experimental

Teórico

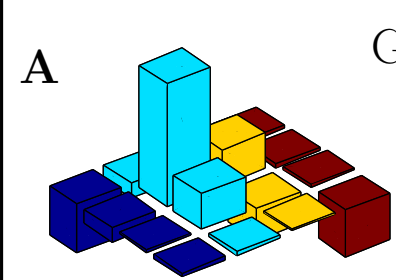

Experimental

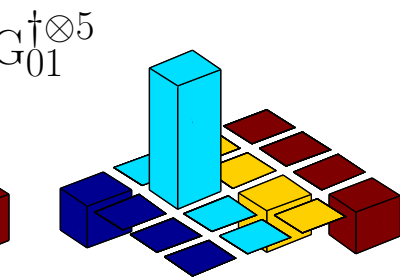

Teórico

Figura 42: Tomografia do algoritmo de Grover para as aparaces $\mathrm{G}_{00}$ e $\mathrm{G}_{01}$. Como as operações implementadas corresponderam na verdade aos operadores inversos $\mathrm{G}^{\dagger}$, as letras $\mathbf{A}, \mathbf{B}, \mathbf{C}, \mathbf{D}$ e $\mathbf{E}$ representam a ordem que seria obtida caso $\mathrm{G}$ de fato fosse aplicado. Estão representadas nesta figura e na próxima somente as componentes reais das respectivas matrizes. As matrizes experimentais completas estão no Apêndice A.7. 


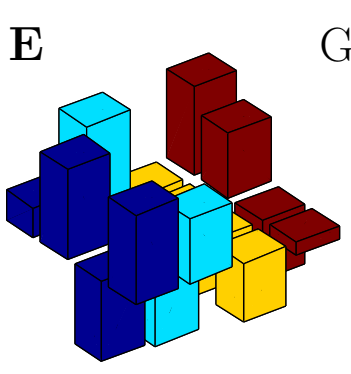

$\mathrm{G}_{10}^{\dagger}$

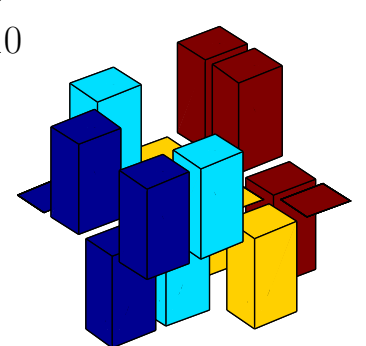

Experimental

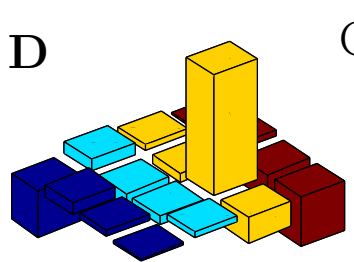

Experimental

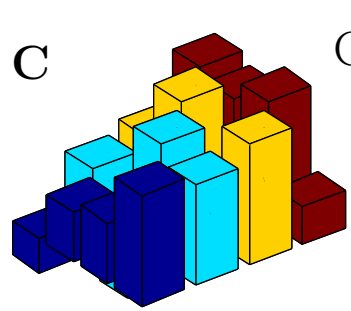

Experimental
$\mathrm{G}_{10}^{\dagger} \otimes 3$

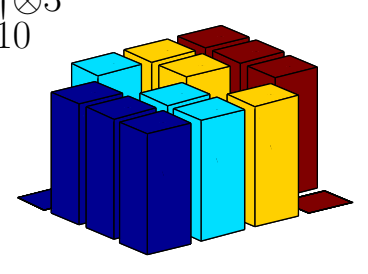

Teórico
$\mathrm{G}_{10}^{\dagger \otimes 2}$

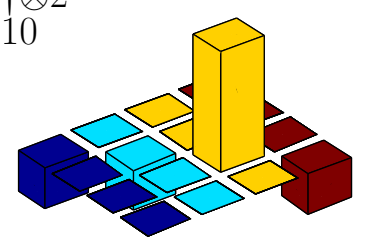

Teórico

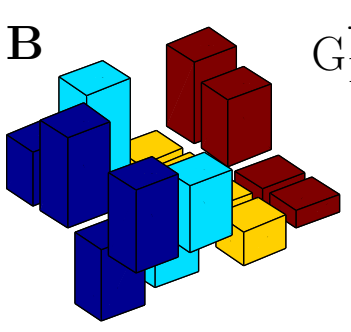

Experimental

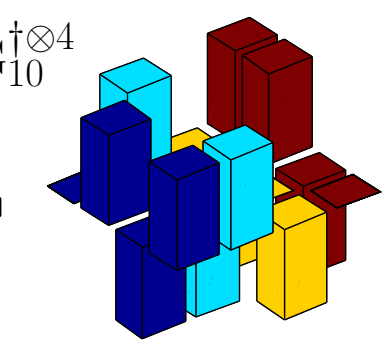

Teórico

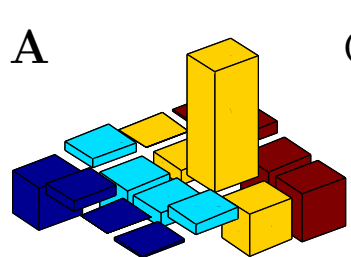

Experimental

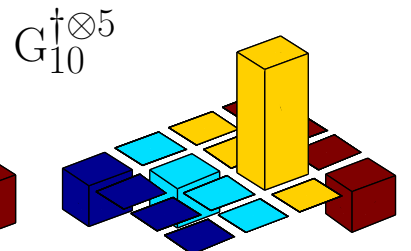

Teórico

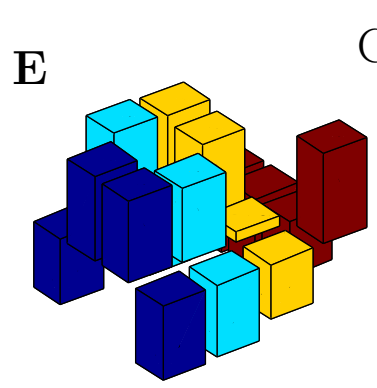

Experimental

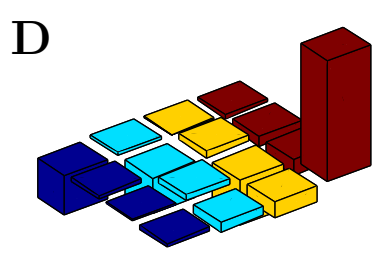

Experimental<smiles>[Al]=[Al]</smiles>

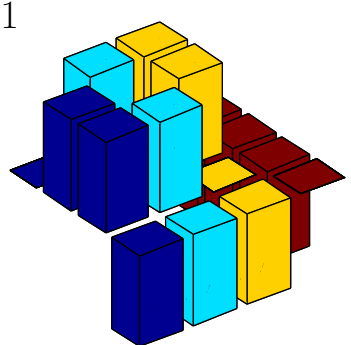

Teórico

$\mathrm{G}_{11}^{\dagger \otimes 2}$

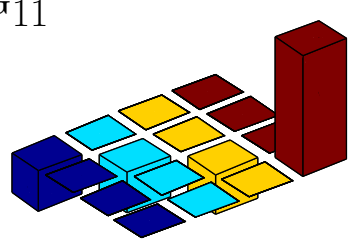

Teórico

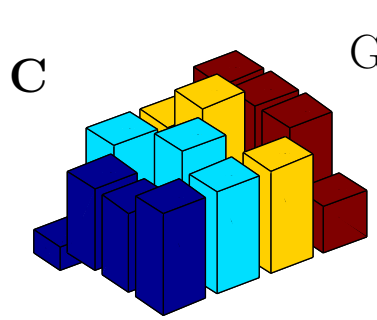

Experimental

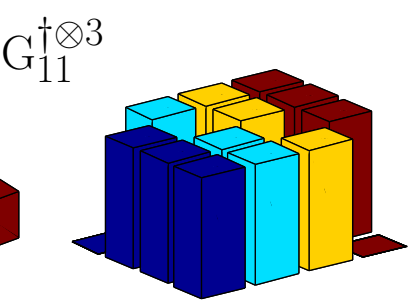

Teórico

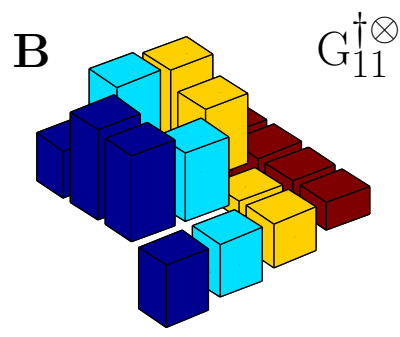

Experimental

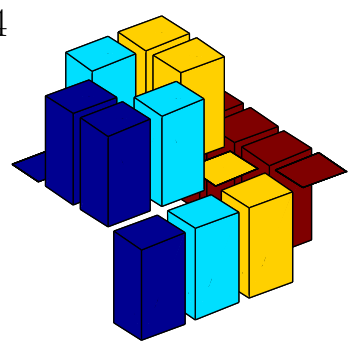

Teórico

A $\mathrm{G}_{11}^{\dagger \otimes 5}$

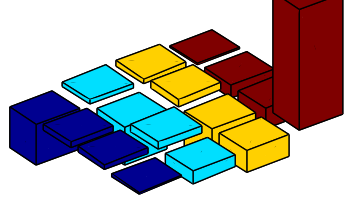

Experimental

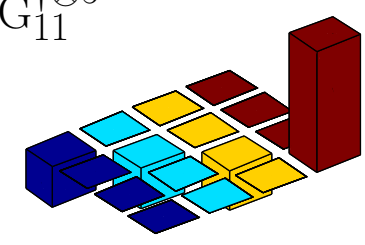

Teórico

Figura 43: Continuação da tomografia do algoritmo de Grover para as operações $\mathrm{G}_{10}$ e $\mathrm{G}_{11}$. 


\subsubsection{Experimentos de Relaxação}

A tomografia da dinâmica de relaxação na amostra de DSS foi realizada sobre 6 diferentes estados iniciais. Para a tomografia dos elementos diagonais, o tempo de relaxação foi estabelecido utilizando-se um tempo de evolução livre, $\tau$, entre o último pulso responsável pela criação do estado e o pulso de leitura da tomografia, conforme a seqüência da Figura 25. Para as coerências, foi utilizado um pulso $\pi$ de refocalização no meio do intervalo de evolução livre. Devido ao fato do pulso $\pi$ ser demasiadamente longo, foi possível observar efeitos de evolução quadrupolar durante a aplicação desse pulso. Esses efeitos foram evidenciados pela oscilação do módulo das coerências ao longo das respectivas curvas de relaxação. Através de simulações numéricas da sequiência de refocalização verificou-se que empregando períodos de evolução livre múltiplos do período quadrupolar, essas oscilações eram bastante reduzidas. Portanto, nas medidas da relaxação de coerências apresentadas nesta seção, os tempos de evolução livre foram todos múltiplos do período quadrupolar.

O primeiro estado medido foi a superposição da Figura 41. Portanto a tomografia experimental dessa figura corresponde a $\tau=0$. A tomografia completa desse estado para $\tau$ varrendo uma faixa de valores igualmente espaçados, desde um valor próximo de 0 até o tempo de retorno ao estado de equilíbrio, encontra-se na Figura 44. Todos os pontos experimentais correspondem ao módulo dos respectivos elementos. Para os experimentos com pulso de refocalização é possível identificar dois grupos de elementos distintos com relação ao tempo de relaxação. $\mathrm{O}$ primeiro é formado pelos elementos que não apresentam oscilação quadrupolar (elementos da diagonal principal e secundária) e o segundo é formado pelos elementos que apresentam essa oscilação (todos os demais elementos). Observa-se claramente que os elementos do primeiro grupo relaxam de forma significativamente mais lenta que os do segundo. Em princípio, podem existir duas contribuições distintas que justificam essa diferença. Uma delas seria a perda de coerência devido à não-refocalização da interação quadrupolar e a outra seria devido aos mecanismos de relaxação que contribuem mais significativamente para os elementos do segundo grupo. Como o objetivo deste trabalho não é a quantificação desses mecanismos, a questão de qual é a relevância relativa dessas duas contribuições fica em aberto.

A Figura 45 contém as medidas das coerências do estado de superposição sem o uso do pulso de refocalização. Nota-se nesse caso que a diminuição nos tempos de relaxação, relativamente ao caso com pulso $\pi$ de refocalização, é proporcional ao quantum magnético de cada elemento, como era esperado para a defasagem devido à interação Zeeman. 
Relaxação dos elementos de $\rho$ para o estado $\mathrm{HD}^{\otimes 2}|00\rangle$ com pulso $\pi$ de refocalização
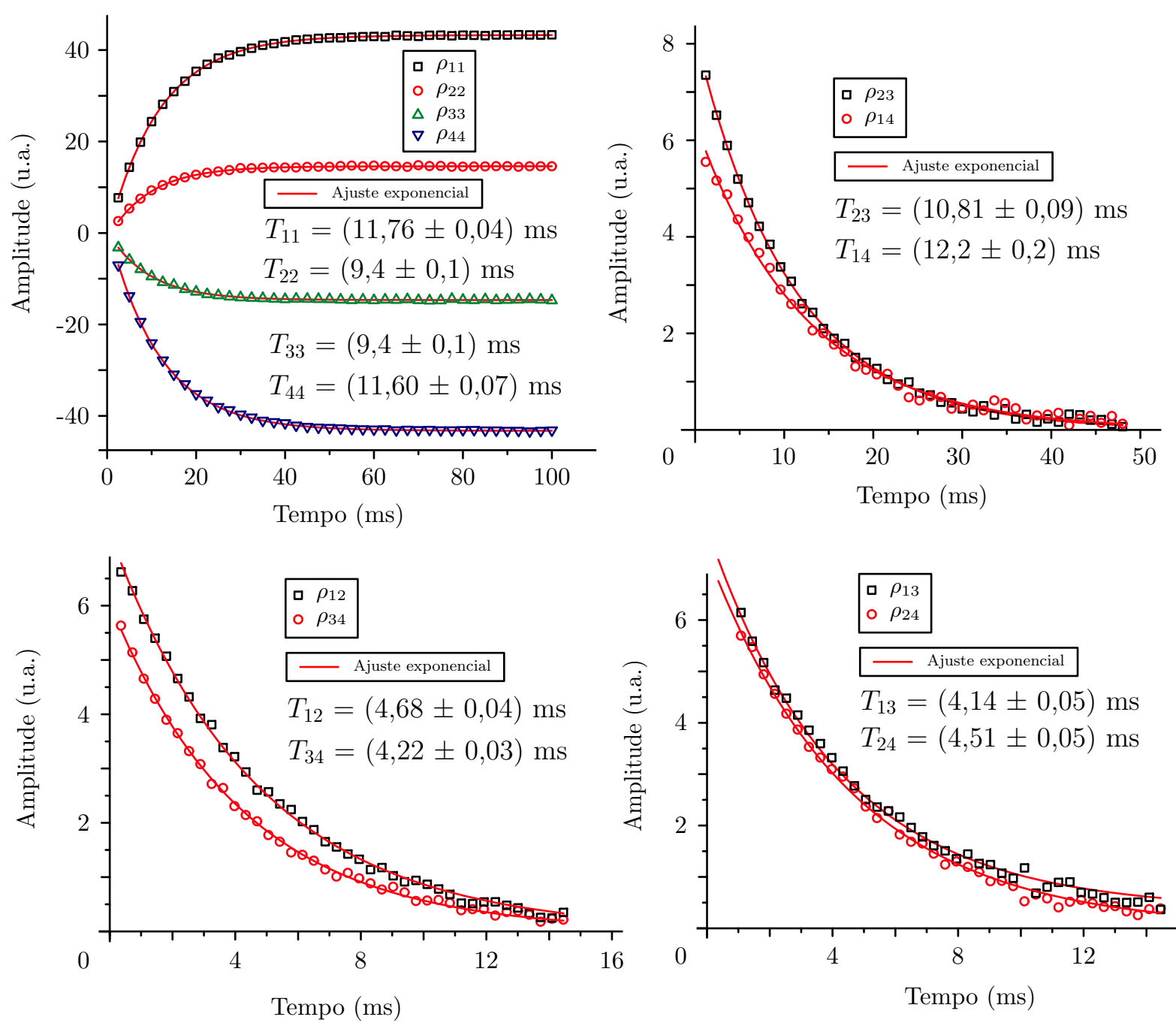

Figura 44: Tomografia da dinâmica de relaxação do estado de superposição da Figura 41 . 
Relaxação dos elementos de $\rho$ para o estado $\operatorname{HD}^{\otimes 2}|00\rangle$ sem pulso de refocalização
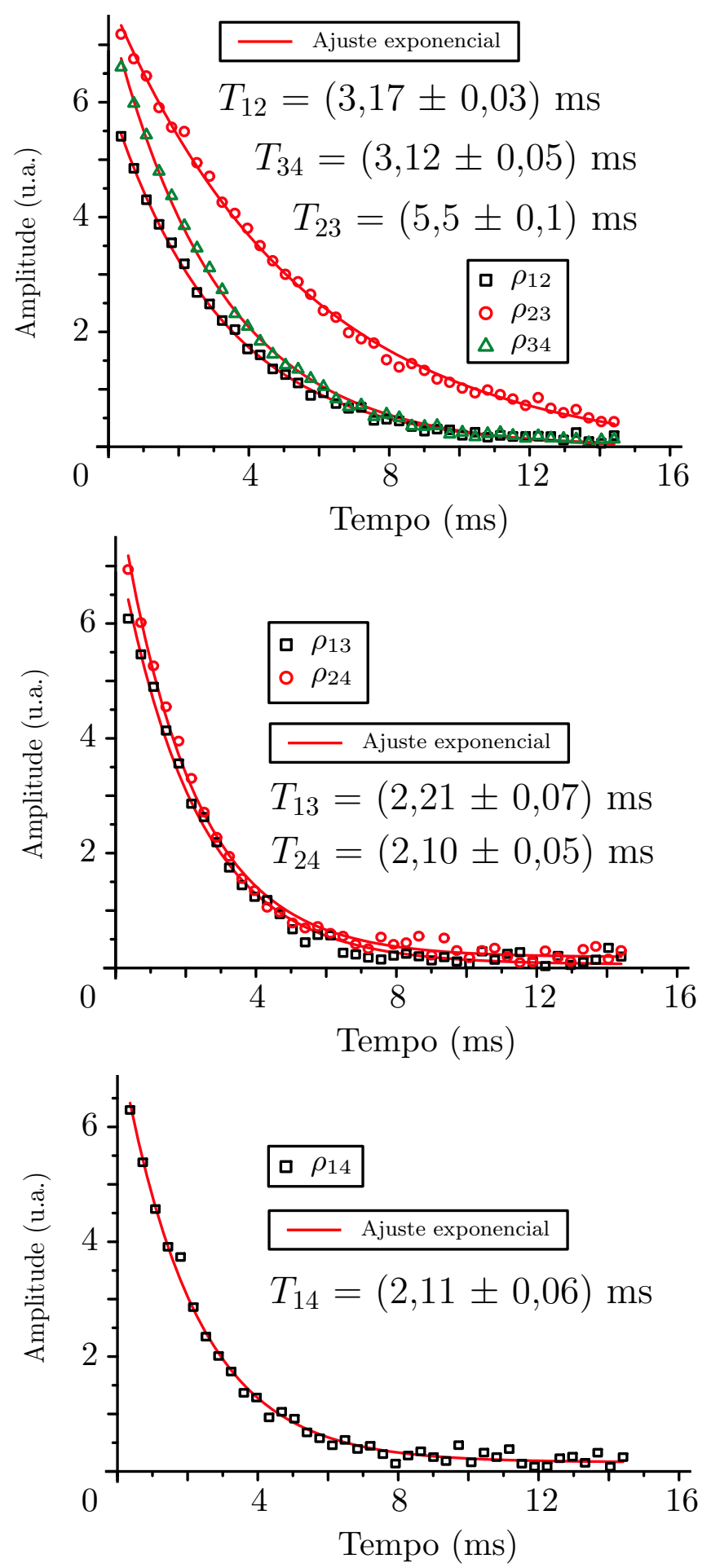

Figura 45: Tomografia da dinâmica de relaxação do estado de superposição da Figura 41 sem pulso de refocalização. Nota-se que nesse caso a diminuição no tempo de relaxação, relativamente ao caso com pulso $\pi$ de refocalização, é proporcional ao respectivo quantum magnético, como era esperado para a defasagem devido à interação Zeeman. 
Os ajustes aos dados experimentais da Figura 44 foram todos mono-exponenciais. Para explicitar esse comportamento, os gráficos da Figura 46 apresentam as relaxações da Figura 44 em escala logarítmica. Percebe-se que o comportamento desses gráficos é bastante linear. Esse resultado corrobora com a aproximação secular para a dinâmica das coerências apresentada na seção 2.3. Isso se deve ao fato da freqüência quadrupolar $(\approx 16 \mathrm{kHz})$ ser bastante superior ao inverso dos tempos de relaxação $(\approx 220 \mathrm{~Hz})$. No entanto, para os elementos diagonais, a teoria de relaxação apresentada na seção 2.3 prevê a possibilidade de um comportamento multi-exponencial. Ainda assim, conforme discutido naquela seção, a evidência de um tal comportamento depende do estado inicial em que o sistema é preparado. Com isso, na tentativa de encontrar a condição experimental para a existência de um ponto de máximo na curva de relaxação é que foram realizadas as tomografias das relaxações dos elementos diagonais dos estados pseudo-puros. Os estados pseudo-puros otimizados nesses experimentos não usaram a medida de fidelidade 2.86 da seção 2.3 , mas sim a diferença quadrática entre os elementos diagonais com relação a estados pseudo-puros teóricos máximos. Estados máximos são aqueles em que as populações assumem o máximo valor possível, o qual é determinado pelas populações de equilíbrio. Note que o fato da fidelidade (2.86) ser normalizada implica na possibilidade de otimização de estados pseudo-puros pouco intensos. Como o objetivo dos experimentos de relaxação é medir a dinâmica até o retorno ao estado de equilíbrio termodinâmico, torna-se interessante maximizar a diferença com relação a esse estado. Para facilitar o processo de otimização, a medida da diferença quadrática foi realizada somente entre os elementos diagonais, fazendo com que os estados gerados possuíssem coerências com valores arbitrários. Devido à aproximação secular, garante-se que a dinâmica de relaxação das populações desses estados não difere em nada da relaxação de pseudo-puros verdadeiros. Por esse motivo, tais estados serão chamados de pseudo-diagonais.

A Figura 47 contém os resultados experimentais da medida de relaxação das populações dos 4 estados pseudo-diagonais da base computacional. É possível observar que a dinâmica de várias populações são claramente multi-exponenciais. A Figura 48 contém a relaxação do estado pseudo-diagonal correspondente ao estado do Gato. De todas as populações mostradas nas Figuras 44, 47 e 48, o elemento $\rho_{11}$ do Gato pseudo-diagonal foi a que apresentou o ponto de máximo mais proeminente. Fazendo-se o ajuste de uma curva bi-exponencial à dinâmica desse elemento, foi possível encontrar dois tempos de relaxação característicos com erros razoavelmente pequenos. Esse resultado fortalece a idéia de que utilizando-se modelos específicos para os mecanismos de relaxação das populações é possível em princípio preparar estados iniciais que maximizem a sensibilidade aos distintos tempos de relaxação do sistema. 
Relaxação do Log das Amplitudes para o estado $\mathrm{HD}^{\otimes 2}|00\rangle$ com pulso $\pi$ de refocalização
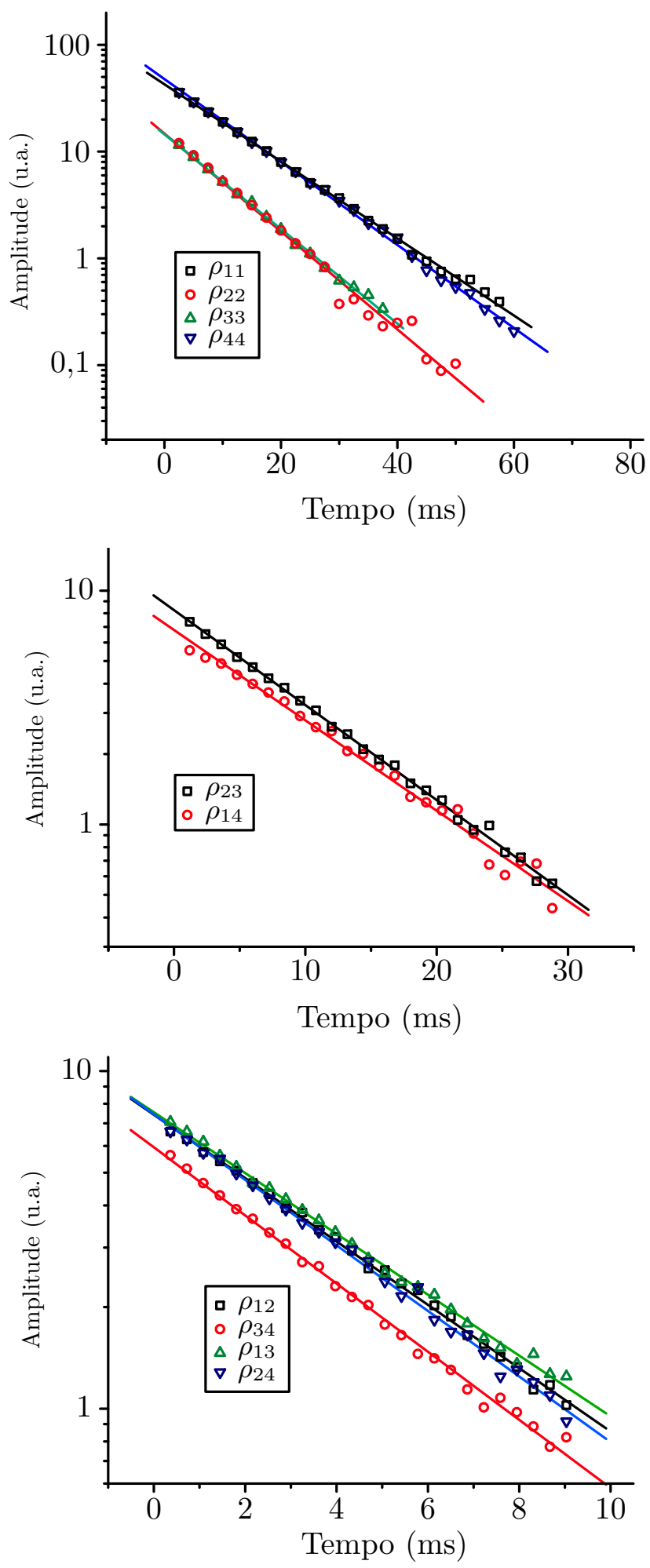

Figura 46: Relaxações da Figura 44 em escala logarítmica. 

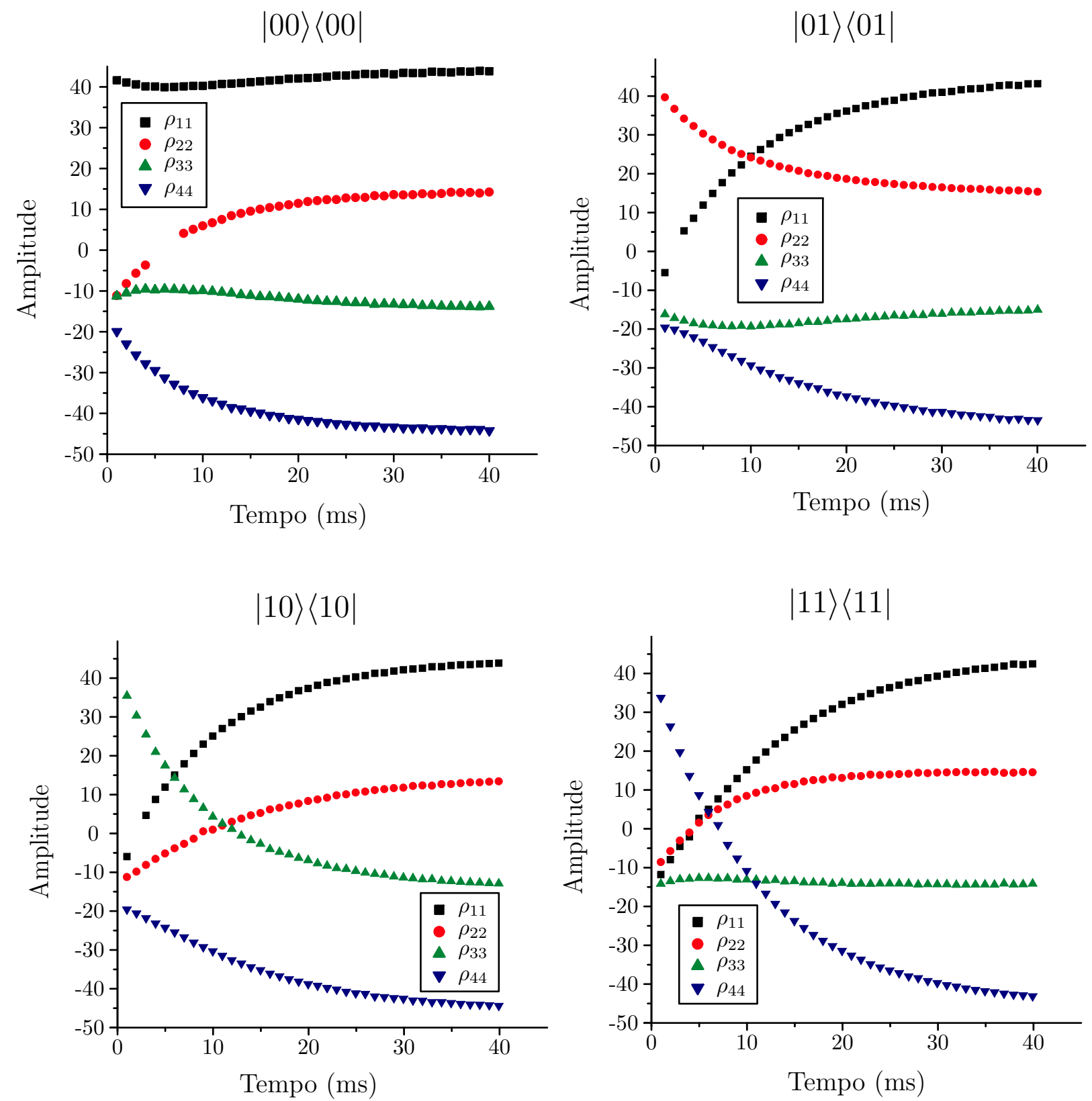

Figura 47: Tomografia da dinâmica de relaxação das populações dos estados pseudo-diagonais correspondentes à base computacional. As interrupções nos pontos experimentais em algumas regiões de amplitudes próximas de 0 ocorreram devido à imprecisão na leitura desses valores. 

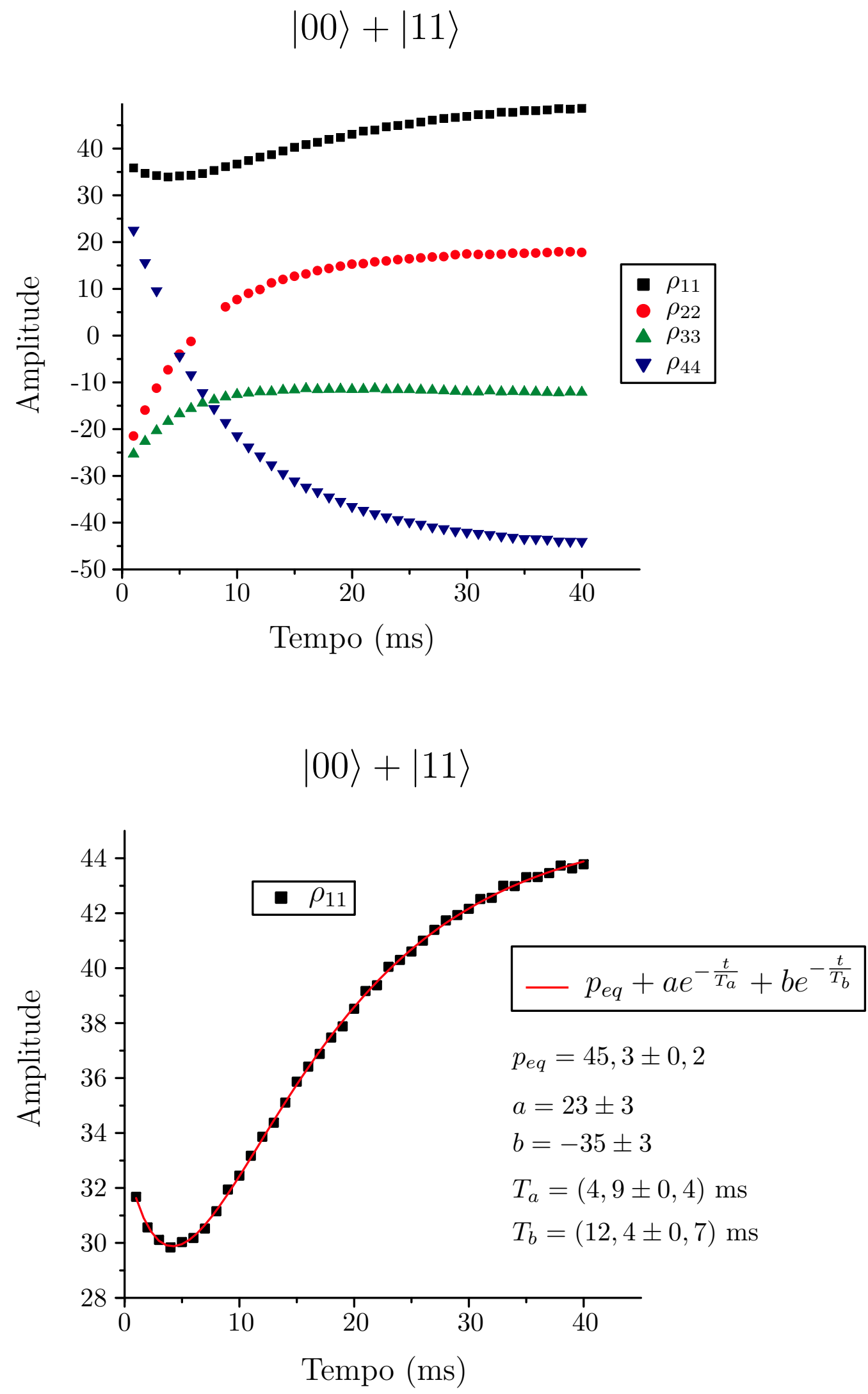

Figura 48: Tomografia da dinâmica de relaxação das populações do estado pseudo-diagonal correspondente ao estado do Gato. 


\section{5}

\section{Conclusões}

O presente trabalho teve como objetivo expor um método de tomografia de estado quântico para ser usado no estudo de implementações de algoritmos e operações quânticas em computação quântica por RMN. Através de uma descrição analítica das rotações produzidas por pulsos de radiofreqüência não-seletivos e pelo uso da base de operadores irredutíveis, foi possível encontrar expressões para a magnetização em termos das funções de Wigner. Explicitando a dependência dos ângulos dos eixos de rotação (fases dos pulsos) na magnetização da amostra, foi possível determinar as combinações de fase necessárias para selecionar uma ordem de coerência específica da matriz densidade. Embora o método de TEQ implementado experimentalmente neste trabalho tenha feito uso do procedimento de seleção de coerências via média temporal, também foi sugerida a realização de seleção via média espacial com gradientes. Foi demonstrado que o método proposto se aplica a qualquer spin isolado. Obviamente, podem haver limitações experimentais devido à queda exponencial na intensidade do sinal medido com o aumento do tamanho do spin nuclear. No entanto, essa é uma limitação intrínseca da técnica de RMN, talvez podendo ser contornada através de técnicas de aumento da polarização dos spins nucleares [38]. Também foi mostrado que a técnica de TEQ pode ser adaptada para sistemas puramente homonucleares. Uma simulação da TEQ em um sistema homonuclear de 3 spins 1/2 acoplados mostrou que, ao menos teoricamente, a tomografia também é bem sucedida para esse tipo de sistema. No entanto, nesses casos existe a necessidade de tempos de evolução livre para a determinação das componentes de rank 0 da matriz densidade. Para sistemas heteronucleares em que só exista um núcleo de cada espécie, o método proposto não apresenta vantagens sobre o trabalho de Long [44], uma vez que rotações individuais em cada núcleo são automaticamente obtidas com pulsos não-seletivos. Para sistemas mistos, em que spins homonucleares e heteronucleares acoplam-se mutuamente, torna-se mais conveniente a utilização de uma base mista para expandir o operador densidade do sistema. Assim, cada grupo homonuclear é expandido por uma base local de tensores irredutíveis $\mathrm{T}_{l m}^{L}$, enquanto que o espaço total da molécula computacional é expandido pela base formada pelo produto tensorial entre os respectivos $\mathrm{T}_{l m}^{L}$. Devido à grande quantidade de interações e ao tamanho do espaço de Hilbert associado, sis- 
temas mistos como esses começam a tornar-se bastante complexos, o que faz com que uma descrição analítica da dinâmica e das transformações necessárias para realizar a TEQ torne-se bastante desafiante. Para esses casos talvez seja mais indicado utilizar técnicas de aquisição multi-dimensional, como por exemplo o método proposto por Das et al. [47] em que se faz uso da transformada de Fourier bidimensional.

Utilizando o procedimento de otimização dos SMP's, foi possível obter altos valores de fidelidade com relação às operações quânticas aplicadas sobre o sistema quadrupolar de spin 3/2. Dessa forma, otimizaram-se SMP's para a criação de estados pseudo-puros correspondentes à base computacional: $|00\rangle,|01\rangle$, $|10\rangle$ e $|11\rangle$ além dos estado do Gato $|00\rangle+|11\rangle$ e do estado de superposição $|00\rangle+|01\rangle+|10\rangle+|11\rangle$. As portas lógicas otimizadas também via SMP's foram a $\mathrm{HD}_{A}, \mathrm{HD}^{\otimes 2}, \mathrm{NOT}_{B}, \mathrm{CN}_{A}$ e $\mathrm{NOT}_{B} \mathrm{CN}_{A}$. Elas foram utilizadas na implementação experimental da criação dos estados de Bell e do algoritmo de Deutsch. Para o algoritmo de busca de Grover foram otimizados diretamente os operadores de Grover para as 4 possíveis respostas de um sistema de 2 q-bits. Todos os resultados obtidos foram satisfatórios, apresentando boas fidelidades experimentais. Em especial é interessante notar que sucessivas aplicações do operador de Grover no algoritmo de busca parecem ter diminuído pouco a fidelidade com relação ao estado de partida.

Com o objetivo de tentar determinar a eficiência do método de TEQ de uma forma independente da fidelidade dos estados quânticos criados, a TEQ foi primeiramente usada para medir a dinâmica de cada elemento da matriz densidade. Uma vez que a hamiltoniana do sistema experimental utilizado é conhecida com alto grau de confiança, esse torna-se um bom teste para a tomografia. Dos resultados obtidos, percebe-se uma excelente concordância com a dinâmica prevista. Também foram realizadas as tomografias de estados criados por sequiências de pulsos simples, as quais por esse motivo também geram estados quânticos mais confiáveis. O estado mais simples tomografado foi o estado de equilíbrio, proporcional ao operador $\mathrm{I}_{z}$, o qual apresentou a fidelidade experimental máxima obtida neste trabalho. O segundo estado mais simples correspondeu à aplicação de um pulso de rotação de $\pi / 2$ que criou o estado proporcional ao operador $\mathrm{I}_{x}$. Por fim foi aplicada uma seqüência de dois pulsos de rotação intercalados por evoluções livres para criar coerências de $2^{a}$ e $3^{a}$ ordens. Das altas fidelidades experimentais obtidas, percebe-se que o método de TEQ foi implementado de forma bem sucedida. Uma verificação experimental da descrição analítica das rotações, foi a medição da dependência da intensidade do sinal da magnetização com o ângulo de nutação imposto pelos pulsos de rotação. Criando-se estados iniciais, via SMP's, proporcionais aos diferentes operadores de polarização, $\mathrm{T}_{l m}$, do spin 3/2, foi possível verificar diretamente a concordância entre a intensidade do sinal com as respectivas funções de Wigner $d_{1, m}^{l}$. Nesse caso, o sinal medido foi obtido aplicando-se 
somente o procedimento de média temporal para a seleção de coerências, não necessitando de um procedimento de reconstrução como é necessário para realizar a TEQ de todos os elementos $\operatorname{de} \rho$.

O método de TEQ também pôde ser aplicado ao estudo da dinâmica de relaxação de todos os elementos de $\rho$ para o núcleo de sódio da amostra de cristal líquido de DSS. Os resultados experimentais da relaxação das coerências de $\rho$ mostraram claramente que a aproximação secular para os diferentes elementos de uma mesma ordem de coerência é válida para esse sistema. Além disso, medindo-se a relaxação das populações para vários estados pseudo-puros, foi possível encontrar estados cujas combinações das populações iniciais puderam evidenciar o comportamento multi-exponencial através da existência de um ponto de máximo para a curva de relaxação.

Entre as futuras aplicações do trabalho desenvolvido neste doutorado, encontra-se a realização experimental da TEQ para o spin 7/2. Atualmente, os estudantes Carlos A. Brasil e Arthur G. de Araújo Ferreira estão desenvolvendo os seus respectivos mestrados no Instituto de Física de São Carlos, tendo como tema a implementação experimental da TEQ e a aplicação de operações quânticas via SMP's na amostra de cristal líquido liotrópico constituída pela molécula de Perfluoroctanato de Césio (CsPFO), a qual foi gentilmente fornecida pelo Prof. Dr. Patrick Judeinstein da Université de Paris-Sud, França. Nesse sistema, núcleos de césio de spin 7/2 são usados como um sistema de 3 q-bits. Uma outra aplicação do método de TEQ pode ser realizada em sistemas homonucleares. Um sistema experimental que pode se beneficiar do método de TEQ é o ácido di-bromopropiônico em solução. Os três hidrogênios dessa molécula constituem um sistema de três q-bits. Devido à baixa diferença de deslocamento químico entre dois dos hidrogênios dessa molécula, pulsos seletivos em um desses spins tornam-se difíceis de serem implementados. Assim, rotações globais via pulsos não-seletivos podem apresentar vantagens para casos como esses. Uma outra possibilidade de tomografia para esse sistema é através da otimização de SMP's que realizem as rotações seletivas, conforme proposto por Fortunato et al. [15]. Deve-se notar que conforme mencionado acima, técnicas analíticas de tomografia começam a tornar-se excessivamente complicadas para um maior número de q-bits. Por exemplo, no caso do spin 3/2 é necessário determinar 9 elementos independentes, para o spin 7/2 são necessários 35 elementos e para um sistema de 4 q-bits seriam necessários 135 elementos. Caso no trabalho de Vandersypen et al. [23] os autores desejassem tomografar a matriz densidade completa, existiriam 8255 elementos a serem determinados!

Outros trabalhos que estão em andamento e que fazem uso do método de TEQ aqui proposto ainda utilizando a amostra de DSS, são os trabalhos de doutorado do estudante Ruben 
Auccaise e de mestrado da estudante Suenne Riguette, ambos realizados no Centro Brasileiro de Pesquisas Físicas em colaboração com o grupo de RMN do IFSC. Entre os estudos realizados por Auccaise estão a descrição dos mecanismos de relaxação responsáveis pelos tempos de relaxação de cada coerência do núcleo de sódio e a simulação quântica de um condensado bosônico. Já o trabalho de mestrado de Riguette consiste na implementação da transformada de Fourier quântica para 2 q-bits.

Com a realização deste trabalho, espera-se que os métodos propostos e todo o sistema experimental descrito e otimizado possam ser usados como uma bancada de testes para estudos de computação e informação quântica principalmente em sistemas de 2 q-bits. Acredita-se que as rotinas de calibração e programação, bem como os algoritmos de otimização aqui descritos, permitem que usuários desse sistema gastem pouco tempo no processo de adaptação experimental dos seus respectivos estudos de interesse. 


\section{Referências}

[1] F. A. Bonk, R. S. Sarthour, E. R. deAzevedo, J. D. Bulnes, G. L. Mantovani, J. C. C. Freitas, T. J. Bonagamba, A. P. Guimaraes, and I. S. Oliveira. Quantum-state tomography for quadrupole nuclei and its application on a two-qubit system. Physical Review A, 69(4), 2004.

[2] R. S. Sarthour, E. R. deAzevedo, F. A. Bonk, E. L. G. Vidoto, T. J. Bonagamba, Guimarães A. P., J. C. C. Freitas, and I. S. Oliveira. Relaxation of coherent states in a two-qubit nmr quadrupole system. Physical Review A, 68:022311, 2003.

[3] F. A. Bonk, E. R. deAzevedo, R. S. Sarthour, J. D. Bulnes, J. C. C. Freitas, A. P. Guimaraes, I. S. Oliveira, and T. J. Bonagamba. Quantum logical operations for spin $3 / 2$ quadrupolar nuclei monitored by quantum state tomography. Journal of Magnetic Resonance, 175(2):226-234, 2005.

[4] J. D. Bulnes, F. A. Bonk, R. S. Sarthour, E. R. deAzevedo, J. C. C. Freitas, T. J. Bonagamba, and I. S. Oliveira. Quantum information processing through nuclear magnetic resonance. Brazilian Journal of Physics, 35(3A):617-625, 2005.

[5] J. Teles, E. R. deAzevedo, R. Auccaise, R. S. Sarthour, I. S. Oliveira, and T. J. Bonagamba. Quantum state tomography for quadrupolar nuclei using global rotations of the spin system. Journal of Chemical Physics, 126:154506, 2007.

[6] J. D. Bulnes. Emaranhamento e Separabilidade para Computação Quântica por Ressonância Magnética Nuclear. Tese de doutorado. Centro Brasileiro de Pesquisas Físicas, 2005.

[7] F. A. Bonk. Tomografia de Estados Quânticos em Sistemas Quadrupolares com spin 3/2: Uma Aplicação da Ressonância Magnética Nuclear à Computação Quântica. Tese de doutorado. Instituto de Física de São Carlos, Universidade de São Paulo, 2005.

[8] I. S. Oliveira, T. J. Bonagamba, R. S. Sarthour, J. C. C. Freitas, and E. R. deAzevedo. NMR Quantum Information Processing. Elsevier, 2007.

[9] D. Reichert and G. Hempel. Receiver imperfections and cyclops: An alternative description. Concepts in Magnetic Resonance, 14(2), 2002.

[10] J. Teles, F. Bonk, E. R. deAzevedo, R. S. Sarthour, I. S. Oliveira, and T. J. Bonagamba. Quantum state tomografhy for spin 7/2 nuclei. In $10^{\text {th }}$ Nuclear Magnetic Resonance Users Meeting Abstracts and Program, page 130. AUREMN, 2005.

[11] D. Suter and J. G. Pearson. Experimental classification of multi-spin coherence under the full rotation group. Chemical Physics Letters, 144(4):328-332, 1988. 
[12] P. J. Grandinetti. Does phase cycling work for nuclei experiencing strong quadrupolar couplings? Solid State Nuclear Magnetic Resonance, 23(1-2):1-13, 2003.

[13] T. J. Bonagamba. Espectroscopia de alta resolução em sólidos por ressonância magnética nuclear. Tese de doutorado. Instituto de Física de São Carlos, Universidade de São Paulo, 1991.

[14] E. R. deAzevedo. Novas metodologias de Ressonância Magnética Nuclear para o Estudo da Dinâmica Lenta em Materiais Orgânicos no Estado Sólido: Aplicações em Polímeros e Proteínas. Tese de doutorado. Instituto de Física de São Carlos, Universidade de São Paulo, 2001.

[15] E. M. Fortunato, M. A. Pravia, N. Boulant, G. Teklemariam, T. F. Havel, and D. G. Cory. Design of strongly modulating pulses to implement precise effective hamiltonians for quantum information processing. Journal of Chemical Physics, 116(17):7599-7606, 2002.

[16] H. Kampermann and W. S. Veeman. Characterization of quantum algorithms by quantum process tomography using quadrupolar spins in solid-state nuclear magnetic resonance. Journal of Chemical Physics, 122(21), 2005.

[17] J. A. Jones. Nmr quantum computing. Progress in Nuclear Magnetic Ressonance Spectroscopy, 38:325-360, 2001.

[18] L. M. K. Vandersypen and I. L. Chuang. Nmr techniques for control and computation. Reviews of Modern Physics, 76:1037-1069, 2004.

[19] A. E. Nielsen and I. L. Chuang. Computação Quântica e Informação Quântica. Porto Alegre: Bookman, 2005.

[20] J. Stolze and D. Suter. Quantum Computing: A Short Course from Theory to Experiment. WILEY-VCH, 2004.

[21] D. P. DiVincenzo. The physical implementation of quantum computation. Fortschritte Der Physik-Progress of Physics, 48(9-11):771-783, 2000.

[22] P. Shor. In Proc. of the 35th Annual Symposium on Foundations of Computer Science. IEEE Computer Society, 1994.

[23] L. M. K. Vandersypen, M. Steffen, G. Breyta, C. S. Yannoni, M. H. Sherwood, and I. L. Chuang. Experimental realization of shor's quantum factoring algorithm using nuclear magnetic resonance. Nature, 414(6866):883-887, 2001.

[24] W. S. Warren. The usefulness of nmr quantum computing. Science, 277:1688-1689, 12 september 1997.

[25] G. Yusa, K. Muraki, K. Takashina, K. Hashimoto, and Y. Hirayama. Controlled multiple quantum coherences of nuclear spins in a nanometre-scale device. Nature, 434(7036):1001-1005, 2005.

[26] R. Tycko. Techniques - nmr on a chip. Nature, 434(7036):966-967, 2005. 
[27] Y. Hirayama, A. Miranowicz, T. Ota, G. Yusa, K. Muraki, S. K. Ozdemir, and N. Imoto. Nanometre-scale nuclear-spin device for quantum information processing. Journal of Physics-Condensed Matter, 18(21):S885-S900, 2006. Sp. Iss. SI.

[28] N. A. Gershenfeld and I. L. Chuang. Bulk spin-resonance quantum computation. Science, 275(5298):350-356, 1997.

[29] D. G. Cory, A. F. Fahmy, and T. F. Havel. Ensemble quantum computing by nmr spectroscopy. Proceedings of the National Academy of Sciences of the United States of America, 94(5):1634-1639, 1997.

[30] A. Abragam. The Principles of Nuclear Magnetism. Oxford University Press, 1978.

[31] A. K. Khitrin and B. M. Fung. Nuclear magnetic resonance quantum logic gates using quadrupolar nuclei. Journal of Chemical Physics, 112(16):6963-6965, 2000.

[32] J. C. Facelli. Calculations of chemical shieldings: Theory and applications. Concepts in Magnetic Resonance Part A, 20A(1):42-69, 2004.

[33] C. P. Slichter. Principles of Magnetic Resonance. Springer-Verlag, 1990.

[34] C. Cohen-Tannoudji, B. Diu, and F. Laloë. Quantum Mechanics, volume I e II. Wiley, 1977.

[35] A. Wokaun and R. R. Ernst. Selective detection of multiple quantum transitions in nmr by 2-dimensional spectroscopy. Chemical Physics Letters, 52(3):407-412, 1977.

[36] S. Vega. Fictitious spin 1/2 operator formalism for multiple quantum nmr. Journal of Chemical Physics, 68(12):5518-5527, 1978.

[37] O. Moussa. On heat-bath algorithmic cooling and its implementation in solid-state nmr. Master's thesis, University of Waterloo, 2005. http://www.icq.ca/ omoussa/work/thesis/.

[38] J. Baugh, O. Moussa, C. A. Ryan, A. Nayak, and R. Laflamme. Experimental implementation of heat-bath algorithmic cooling using solid-state nuclear magnetic resonance. Nature, 438(7067):470-473, 2005.

[39] E. O. Stejskal and J. D. Memory. High Resolution NMR in the Solid State. Oxford University Press, 1994.

[40] J. A. Nelder and R. Mead. Comput. J., 7:308, 1965. (UK).

[41] W. H. Press, B. P. Flannery, S. A. Teukolsky, and W. T. Vetterling. Numerical Recipes in FORTRAN: The Art of Scientific Computing. Cambridge University Press, 1992.

[42] S. A. Smith, W. E. Palke, and J. T. Gerig. The hamiltonians of nmr. part iv: Nmr relaxation. Concepts in Magnetic Resonance, 6:137-162, 2002.

[43] I. L. Chuang, N. Gershenfeld, M. G. Kubinec, and D. W. Leung. Bulk quantum computation with nuclear magnetic resonance: theory and experiment. Proceedings of the Royal Society of London Series a-Mathematical Physical and Engineering Sciences, 454(1969):447-467, 1998. 
[44] G. L. Long, H. Y. Yan, and Y. Sun. Analysis of density matrix reconstruction in nmr quantum computing. Journal of Optics B-Quantum and Semiclassical Optics, 3(6):376-381, 2001.

[45] I. L. Chuang, N. Gershenfeld, and M. Kubinec. Experimental implementation of fast quantum searching. Physical Review Letters, 80(15):3408-3411, 1998.

[46] H. Kampermann and W. S. Veeman. Quantum computing using quadrupolar spins in solid state nmr quantum information processing. Quantum Information Processing, 1:327 344, 2002.

[47] R. Das, T. S. Mahesh, and A. Kumar. Efficient quantum-state tomography for quantum-information processing using a two-dimensional fourier-transform technique. Physical Review A, 67(6), 2003.

[48] D. A. Varshalovich, A. N. Moskalev, and V. K. Khersonskii. Quantum theory of angular momentum. World Scientific Pub, Singapore; Teaneck, NJ, USA, 1988.

[49] M. E. Rose. Elementary Theory of Angular Momentum. John Wiley, 1957.

[50] A. R. Edmonds. Angular Momentum in Quantum Mechanics. Princeton Univ. Press, 1957.

[51] R. N. Zare. Angular Momentum: Understanding Spatial Aspects in Chemistry and Physics. John Wiley, 1988.

[52] B. C. Sanctuary. Multipole operators for an arbitrary number of spins. Journal of Chemical Physics, 64(11):4352-4361, 1976.

[53] G. Bodenhausen, H. Kogler, and R. R. Ernst. Selection of coherence-transfer pathways in nmr pulse experiments. Journal of Magnetic Resonance, 58(3):370-388, 1984.

[54] A. D. Bain. Coherence levels and coherence pathways in $\mathrm{nmr}$ - a simple way to design phase cycling procedures. Journal of Magnetic Resonance, 56(3):418-427, 1984.

[55] C. L. Khetrapal, A. C. Kunwar, A. S. Tracey, and P. Diehl. Nuclear Magnetic Resonance Studies in Lyotropic Liquid Crystals. Springer-Verlag, 1975.

[56] J. Teles, C. E. Garrido, and A. Tannús. A convenient procedure for magnetic field homogeneity evaluation. Journal of Physics D: Applied Physics, 37:1877-1880, 2004.

[57] R. F. Turchiello and E. A. Oliveira. Behavior of the director reorientation time in glass surfaces of lyotropic liquid crystals in the nematic to biaxial transition. Physical Review E, 54(2):1618-1624, 1996.

[58] L. M. Vega, J. J. Bonvent, G. Barbero, and E. A. Oliveira. Critical orientational states in lyotropic liquid crystals induced by a magnetic field. Physical Review E, 57(4):R3715-R3718, 1998.

[59] Varian Associates Inc. VNMR User Programming, VNMR 6.1B Software, 1998. Pub. No. 01-999088-00 A1298.

[60] R. Kyburz. VNMR Pulse Sequences, Programming and Hardware Aspects. Varian Associates Inc., 1998. Pub. No. 01-999014-00, Rev. A0398. 


\section{A Apêndices}

\section{A.1 Descrição didática do método de TEQ}

Com o intuito de tornar mais claro os objetivos e conteúdos desta tese, é feita aqui uma exposição mais didática do método de tomografia de estado quântico. A preocupação neste apêndice não é prover uma apresentação rigorosa do método, mas sim descrever os princípios básicos de funcionamento que o tornam útil para os experimentos de CQ por RMN. Conseqüentemente, os conceitos e explicações são um tanto superficiais. Para facilitar a compreensão, é descrita somente a TEQ do núcleo de spin 3/2. No entanto, esse é um exemplo importante tratado nesta tese e que serve para ilustrar as principais idéias do método.

Para iniciar a exposição, será analisado o caso simples e um tanto idealizado de um computador quântico cujo estado pode ser descrito por um estado puro. A Figura 49 a ilustra a atuação de um algoritmo quântico sobre o estado de entrada $\left|\psi_{0}\right\rangle$. Como saída, obtém-se o estado $|\psi\rangle$. Assumindo por simplicidade que a base computacional é a base de auto-vetores do operador de medida, medidas diretas sobre $|\psi\rangle$ possibilitam obter apenas o módulo das amplitudes do estado: $\left|a_{0}\right|, \ldots,\left|a_{7}\right|$, vide Figura 49 b. Em geral, os algoritmos quânticos são projetados para que medidas feitas diretamente nessa base sejam suficientes para obter as vantagens computacionais sobre os equivalentes clássicos. No entanto, quando o objetivo é determinar propriedades gerais do processamento de tais algoritmos, torna-se interessante obter a informação completa do estado $|\psi\rangle$. Para isso, é necessário obter, adicionalmente, os valores das fases das amplitudes $a_{i}$. A maneira de obter essa informação é aplicando uma transformação unitária que produza combinações das amplitudes originais, das quais é possível extrair o valor das fases relativas entre os diferentes auto-estados. As Figuras 49. c e 49.d ilustram esse processo. Por motivos didáticos, considerou-se uma transformação simples, cuja amplitude resultante para o primeiro auto-estado é igual a soma das amplitudes iniciais dos dois primeiros auto-estados. Evidentemente, transformações mais gerais podem ser produzidas. Ainda assim, esse exemplo ilustra satisfatoriamente o efeito de interferência quântica utilizado para a caracterização completa do estado. 


\section{Estados Puros}

\section{Medida na base computacional}

a)

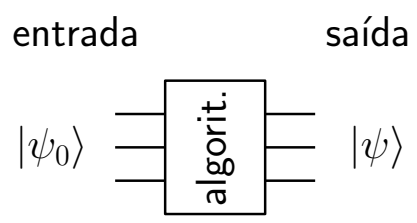

b)

$$
\overbrace{\left|a_{0}\right|^{2}\left|a_{1}\right|^{2} \cdots\left|a_{7}\right|^{2}}^{|\psi\rangle=a_{0}|000\rangle+\ldots+a_{7}|111\rangle}
$$

\section{Mudança de base}

c)

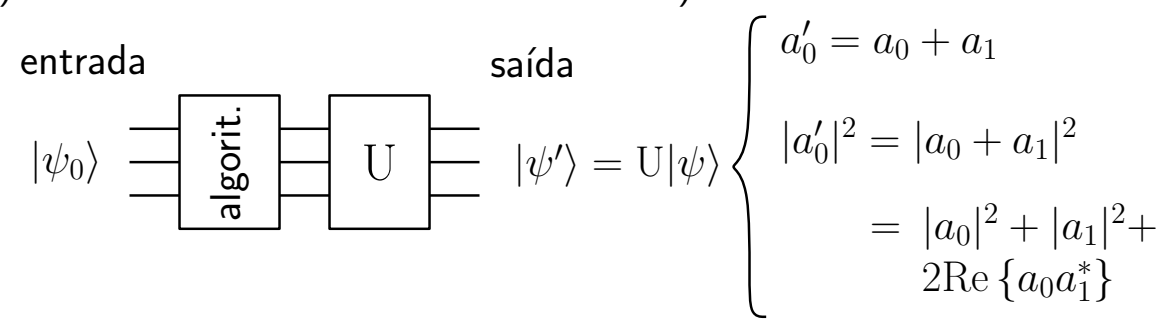

Figura 49: Ilustração do processo de caracterização de um estado quântico puro.

O caso da caracterização de um estado puro, embora útil conceitualmente, não corresponde em geral aos casos experimentais, em que o estado do sistema é conhecido apenas de maneira estatística. Nesses casos, a descrição mais completa é através do operador densidade $\rho$. A Figura 50,a ilustra a representação matricial do operador densidade na base computacional. Nesse caso é representado o estado de dois q-bits. Medidas nessa base fornecem somente os valores das chamadas populações, que são os elementos diagonais de $\rho$. Para obter o valor das coerências (elementos não-diagonais), aplicam-se transformações unitárias de maneira análoga ao caso puro do parágrafo anterior. A Figura 50,b ilustra esse processo. Conhecendo-se os elementos $b_{r s}$ da matriz de transformação U é possível relacionar os elementos originais $\rho_{i j}$ com os transformados $\rho_{i j}^{\prime}$. Assim, as medidas dos novos elementos diagonais $\rho_{i i}^{\prime}$ fornecem a informação sobre as coerências originais $\rho_{i j}$. A essência dos métodos de tomografia é, portanto, encontrar um conjunto de transformações que permita determinar todos os elementos de $\rho$. A escolha dessas transformações está obviamente condicionada à técnica experimental empregada. Em muitos casos, somente tipos particulares de transformações podem ser utilizadas devido às restrições na hamiltoniana de controle do sistema. Fatores como, tempo de decoerência, minimização de erros e sensibilidade à desvios de parâmetros experimentais também devem ser levados em conta na escolha do método de TEQ.

No caso da TEQ aplicada à sistemas de RMN, a base computacional utilizada coincide com a base de auto-vetores da hamiltoniana principal, a qual é proporcional ao operador $\mathrm{I}_{z}$. Já o 


\section{Estados Mistos}

$\rho=\left[\begin{array}{cccc}\mathbf{0 0} & \mathbf{0 1} & \mathbf{1 0} & \mathbf{1 1} \\ \rho_{11} & \rho_{12} & \rho_{13} & \rho_{14} \\ \rho_{12}^{*} & \rho_{22} & \rho_{23} & \rho_{24} \\ \rho_{13}^{*} & \rho_{23}^{*} & \rho_{33} & \rho_{34} \\ \rho_{14}^{*} & \rho_{24}^{*} & \rho_{34}^{*} & \rho_{44}\end{array}\right] \begin{array}{ll}\mathbf{0 0} & \\ \mathbf{0 1} & \rho_{i i}=P_{i}=\operatorname{Tr}\left\{\rho\left|u_{i}\right\rangle\left\langle u_{i}\right|\right\} \\ \mathbf{1 1} & \end{array}$

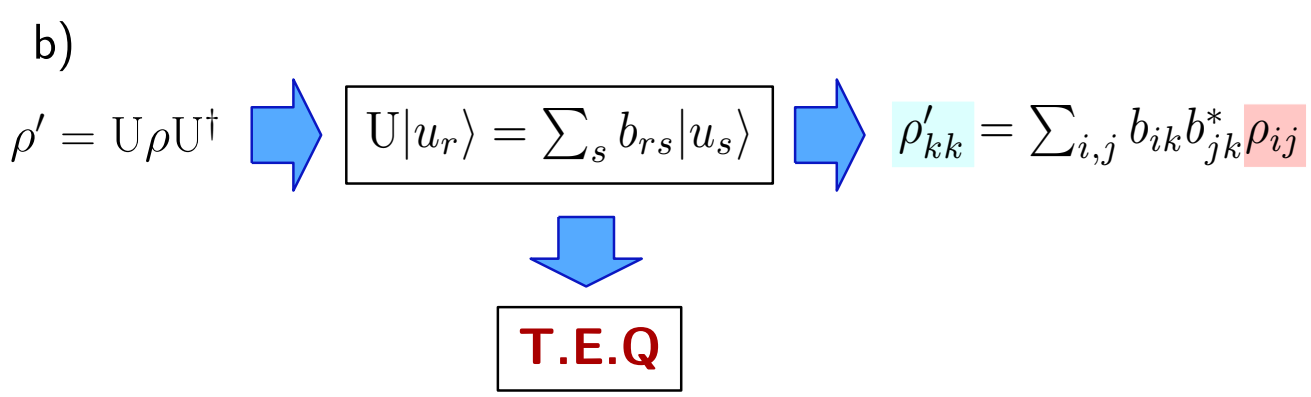

Figura 50: Ilustração do processo de caracterização de um estado quântico misto. A escolha conveniente das transformações U determina a eficiência do método.

operador de medida corresponde a $\mathrm{I}_{x}$ ou $\mathrm{I}_{y}$. Portanto, medidas diretas em RMN fornecem o valor de um conjunto específico de coerências. Como o operador levantamento $\mathrm{I}_{+}$é formado pela combinação dos operadores $\mathrm{I}_{x}$ e $\mathrm{I}_{y}$, os elementos medidos são chamados de coerências de primeira ordem. Essa designação se deve ao fato de que $I_{+}$eleva em um quantum os autoestados de $\mathrm{I}_{z}$. A região vermelha na matriz $\rho$ na Figura 51 , a engloba esses elementos de primeira ordem para o caso de um núcleo com spin 3/2. Para um único núcleo de spin $I$, as coerências de $n$-ésima ordem estão sempre contidas na super-diagonal formada pelos elementos $\rho_{i, i+n}$.

Conforme discutido na Introdução, o método de TEQ proposto neste trabalho teve como ponto de partida o trabalho de Bonk et al. [1]. Nesse caso, os autores observaram que a transformação correspondente à técnica CYCLOPS [9] produz elementos de primeira ordem $\rho_{k, k+1}^{\prime}$ dependentes somente dos elementos diagonais. O sistema de equações gerado por essa transformação permite determinar todos os elementos diagonais $\rho_{i i}$ da matriz original. A Figura 51. b ilustra esse processo. As transformações $\mathrm{U}_{R_{\mu}}$ utilizadas pela técnica CYCLOPS são obtidas através de pulsos de campo magnético com duração bastante curta, cujo efeito é produzir rotações globais do spin nuclear. Esses pulsos são também chamados de não-seletivos. A vantagem desse tipo de transformação se deve não só à rapidez com que ela é implementada mas também à simplicidade e robustez da forma do pulso correspondente.

Para obter os elementos não-diagonais de $\rho$, Bonk et al. propuseram o emprego dos chamados pulsos seletivos, os quais possuem a propriedade de causar a transferência entre coerências 


\section{Tomografia para $I=3 / 2$}
a)
$\rho=\left[\begin{array}{cccc}\rho_{11} & \rho_{12} & \rho_{13} & \rho_{14} \\ \rho_{12}^{*} & \rho_{22} & \rho_{23} & \rho_{24} \\ \rho_{13}^{*} & \rho_{23}^{*} & \rho_{33} & \rho_{34} \\ \rho_{14}^{*} & \rho_{24}^{*} & \rho_{34}^{*} & \rho_{44}\end{array}\right]$
c) Pulsos seletivos
b) Ciclagem CYCLOPS
(pulsos não-seletivos)

$$
\begin{gathered}
\rho^{\prime}=\frac{1}{4} \sum_{\mu=1}^{4} \mathrm{U}_{R_{\mu}} \rho \mathrm{U}_{R_{\mu}}^{\dagger} e^{i \alpha_{\mu}} \\
\rho_{k, k+1}^{\prime}=\sum_{i=1}^{4} b_{i k} \rho_{i i}
\end{gathered}
$$$$
\rho=\left[\begin{array}{cccc}
\rho_{11} & \rho_{12} & \rho_{13} & \rho_{14} \\
\rho_{12}^{*} & \rho_{22} & \rho_{23} & \rho_{24} \\
\rho_{13}^{*} & \rho_{23}^{*} & \rho_{33} & \rho_{34} \\
\rho_{14}^{*} & \rho_{24}^{*} & \rho_{34}^{*} & \rho_{44}
\end{array}\right]
$$$$
\rho^{\prime}=\frac{1}{4} \sum_{\mu=1}^{4} \mathrm{U}_{R_{\mu}} \mathrm{U}_{S} \rho \mathrm{U}_{S}^{\dagger} \mathrm{U}_{R_{\mu}}^{\dagger} e^{i \alpha_{\mu}}
$$

Figura 51: Ilustração do método de tomografia proposto por Bonk et al. [1].

específicas da matriz densidade. Esse tipo de transformação está representada pelo operador $U_{S}$ na Figura 51.c. Assim, através da transferência sucessiva das coerências originais para a posição das populações, foi possível reconstruir a matriz original completa empregando-se a técnica CYCLOPS.

O objetivo do presente trabalho foi generalizar a técnica de TEQ anterior, a qual é específica para núcleos de spin 3/2. No caminho para tal generalização, demonstrou-se que é possível fazer a transferência de todas as coerências e populações de $\rho$ diretamente para a posição das coerências de primeira ordem. Isso para qualquer spin e empregando-se somente pulsos nãoseletivos como os da técnica CYCLOPS. Foi possível demonstrar que o sistema de equações obtido das transformações $\mathrm{U}_{R_{\mu}}$, expostas na Figura 52, permite a determinação da matriz densidade completa. $\mathrm{O}$ número de pulsos $N$ e os demais parâmetros que não estão representados na figura são funções do número quântico de spin, vide Equação (3.40).

Com isso, encerra-se este apêndice. Para um entendimento mais completo do método, aconselha-se a leitura dos principais capítulos da tese. Uma característica importante do método que não foi apresentada aqui, está na possibilidade de adaptação do mesmo (com algumas modificações) a sistemas de spins acoplados. 
Tomografia com pulsos não-seletivos

$$
\rho^{\prime}=\frac{1}{N} \sum_{\mu=1}^{N} \mathrm{U}_{R_{\mu}} \rho \mathrm{U}_{R_{\mu}}^{\dagger} e^{i \alpha_{\mu}} \quad \rho=\left[\begin{array}{cccc}
\rho_{11} & \rho_{12} & \varrho_{13} & \varrho_{14} \\
\rho_{12}^{*} & \rho_{22} & \rho_{23} & \rho_{24} \\
\rho_{13}^{*} & \rho_{23}^{*} & \rho_{33} & \rho_{34} \\
\rho_{14}^{*} & \rho_{24}^{*} & \rho_{34}^{*} & \rho_{44}
\end{array}\right]
$$

Figura 52: Ilustração do método de tomografia proposto nesta tese e que utiliza somente pulsos não-seletivos de curta duração.

\section{A.2 Códigos de otimização dos SMP's}

O código a seguir, escrito em MatLab, realiza a otimização das portas lógicas via pulsos modulados através da minimização da função objetivo utilizando-se um algoritmo simplex. $\mathrm{O}$ conteúdo subjacente a esse código encontra-se discutido nas seções $2.2 \mathrm{e} 4.2$

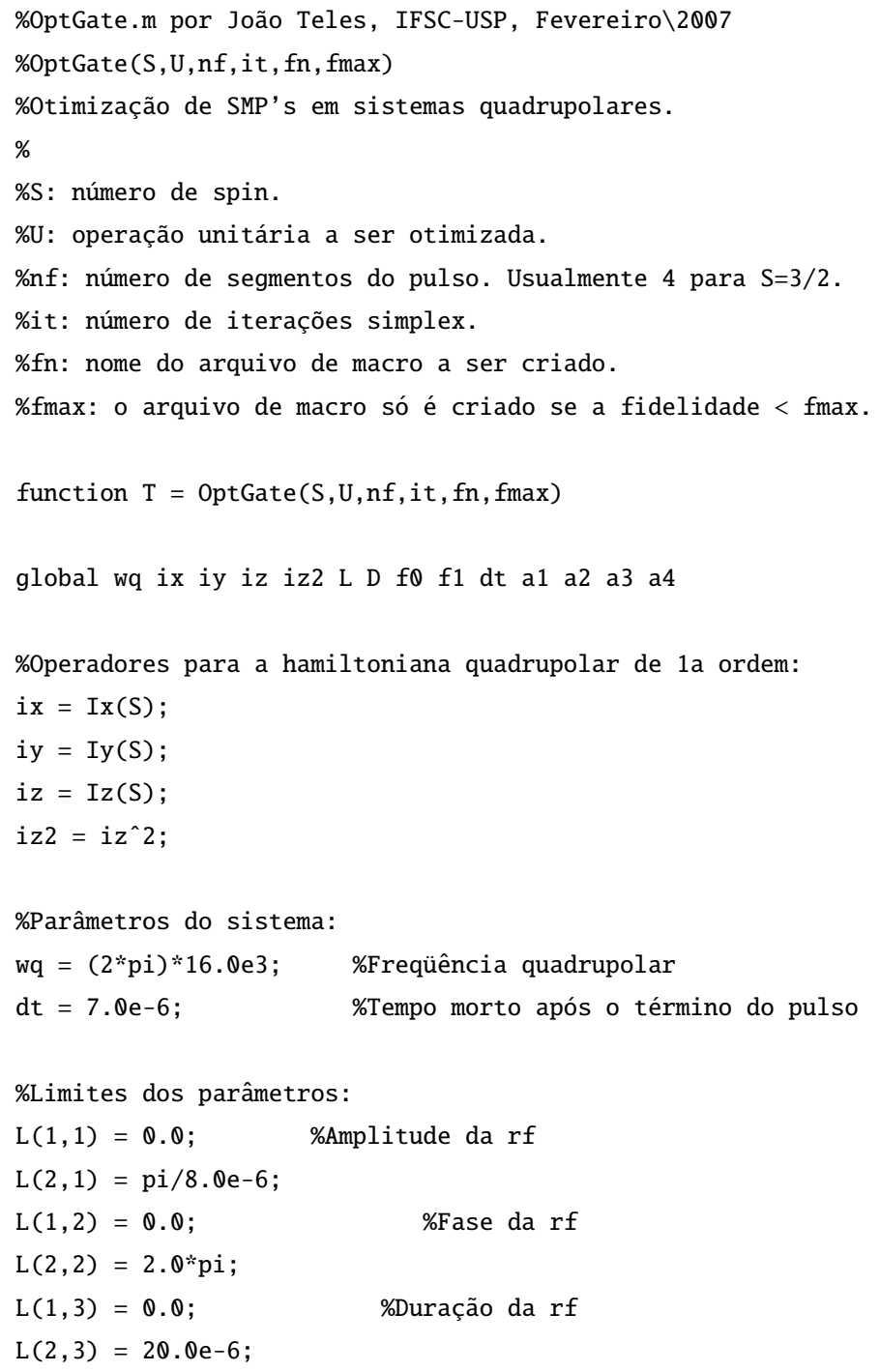


\%Erros dos parâmetros:

\begin{tabular}{|c|c|}
\hline DwQ $=(2 * \mathrm{pi}) * 10 ;$ & \%Erro na freqüência \\
\hline $\mathrm{Dfa}=(\mathrm{pi} / 180) * 2 ;$ & \%Erro na fase \\
\hline Dw1 = 0.05; & \%Erro relativo na amplitude \\
\hline Dts $=0.3 e-6$ & \%Erro no tempo \\
\hline Dwq $=(2 * p i) * 70$ & \%Erro na freqüência quadrupolar \\
\hline
\end{tabular}

\%Valores iniciais gerados aleatoriamente:

$A=\operatorname{zeros}(n f, 3)$;

for $r=1: n f$

$\mathrm{A}(\mathrm{r}, 1)=\mathrm{L}(1,1)+\operatorname{rand}(1) *(\mathrm{~L}(2,1)-\mathrm{L}(1,1)) ;$

$\mathrm{A}(\mathrm{r}, 2)=\mathrm{L}(1,2)+\operatorname{rand}(1) *(\mathrm{~L}(2,2)-\mathrm{L}(1,2)) ;$

$A(r, 3)=L(1,3)+\operatorname{rand}(1) *(L(2,3)-L(1,3))$;

end

\%Rotina de implementação do simplex:

MFE $=20 * n f * 20 \theta$;

\%MaxFunEvals -default $=3 * n f * 200$;

MI $=20 * \mathrm{nf} * 200$;

\%MaxIter -default $=3 * n{ }^{*} 200$;

$\mathrm{B}=\mathrm{A}$;

fbest $=1.0$;

for $\mathrm{p}=1: \mathrm{it}$

$[\mathrm{x}, \mathrm{fval}]=$ fminsearch $(@(\mathrm{x})$ Fid $(\mathrm{x}, \mathrm{U}, \mathrm{S}, \mathrm{nf}), \mathrm{A}$, optimset('MaxFunEvals', MFE, 'MaxIter', MI))

if fval < fbest

$\mathrm{B}=\mathrm{x}$;

fbest $=$ fval;

$\mathrm{fQ}$

f1

if fbest < fmax

$\operatorname{Macro}(S, B, D t s, w q, n f, f n)$;

end

end

\%Busca convergente

$\mathrm{d}=1 ; \% f v a l^{\wedge} 2 ; \quad$ \%Taxa de convergência

for $r=1: n f$

$\mathrm{A}(\mathrm{r}, 1)=\mathrm{B}(\mathrm{r}, 1)+(\mathrm{L}(1,1)+\mathrm{rand}(1) *(\mathrm{~L}(2,1)-\mathrm{L}(1,1))-\mathrm{B}(\mathrm{r}, 1)) * \mathrm{~d} ;$
$\mathrm{A}(\mathrm{r}, 2)=\mathrm{B}(\mathrm{r}, 2)+(\mathrm{L}(1,2)+\mathrm{rand}(1) *(\mathrm{~L}(2,2)-\mathrm{L}(1,2))-\mathrm{B}(\mathrm{r}, 2)) * \mathrm{~d} ;$
$\mathrm{A}(\mathrm{r}, 3)=\mathrm{B}(\mathrm{r}, 3)+(\mathrm{L}(1,3)+\operatorname{rand}(1) *(\mathrm{~L}(2,3)-\mathrm{L}(1,3))-\mathrm{B}(\mathrm{r}, 3)) * \mathrm{~d} ;$

end

melhor $=$ fbest

end

time $=\operatorname{sum}(B(:, 3))$

$\mathrm{T}=\mathrm{B}$;

end 
\%Escrita da macro:

function $\operatorname{Macro}(S, B, D t s$, wq, $\mathrm{nf}, \mathrm{fn})$

global fo f1 a1 a2 a3 a4

$\mathrm{pw}=\operatorname{sum}(\mathrm{B}(:, 3))$;

$\operatorname{maxf1}=\max (\operatorname{abs}(B(:, 1)))$;

filename $=\left[{ }^{\prime} C: \backslash\right.$ Documents and Settings $\backslash j$ teles $\backslash$ Meus documentos $\backslash$ Programas $\backslash$ Varian $\backslash$ Macros $\backslash$ ', fn $]$;

filename $=$ [filename, ' $f f$ ', num2str $(\operatorname{round}(1 \mathrm{e} 4 *(1-f()))$, ' $-d$ ', num2str $(\operatorname{round}(1 \mathrm{e} 4 *(f 1)))$, 'e-4'];

out = fopen(filename, 'w');

fprintf(out, '"Gate fidelity $=\% \mathrm{f} " \backslash \mathrm{n}$ ', $1-\mathrm{fQ}$ );

fprintf(out, ,"Total deviation $=\% f^{\prime \prime} \backslash n \backslash n$ ', f1);

fprintf(out, , "w1*t dev. = \%f" \n', $\operatorname{sqrt}(a 1) /(2 * S+1)$ );

fprintf(out, '"phi dev. = \%f"\n', $\operatorname{sqrt}(a 2) /(2 * S+1)$ );

fprintf(out, ' "(wq/2)*t dev. = \%f" $\backslash n$ ', $\operatorname{sqrt}(a 3) /(2 * \mathrm{~S}+1)$ );

fprintf(out, ' "wQ*t dev. = \%f" \n\n', $\operatorname{sqrt}(a 4) /(2 * S+1)$ );

fprintf(out, 'av $\backslash n \backslash n$ ');

fprintf(out, 'cr= $f 1 \backslash n n l \backslash n n l \backslash n n l \backslash n n l \backslash n n l \backslash n f 1=c r \backslash n \backslash n$ ') ;

fprintf(out, 'cr= $f 2 \backslash n n l \backslash n n l \backslash n n l \backslash n n l \backslash n n l \backslash n \backslash n$ ') ;

fprint $f$ (out, ' $c r=f 3 \backslash n n l \backslash n n l \backslash n n l \backslash n n l \backslash n n l \backslash n f 3=c r \backslash n \backslash n$ ') ;

fprintf(out, 'ph $\backslash n \backslash n$ ');

fprintf(out, '\$wq= 2.0*3.14159265*(f3-f1)/2.0 $\backslash \mathrm{n} \backslash \mathrm{n}^{\prime}$ );

fprintf(out, ' $\$ p w b=\% f e 6 / \$ w q \backslash n$ ', wq*pw);

fprintf(out, '\$pwa= $0.0 \backslash n \backslash n$ ');

fprintf(out, 'write(' 'reset', , ',/export/home/CQ/vnmrsys/shapelib/\%s.RF' ') \n', fn);

for $\mathrm{r}=1: \mathrm{nf}$

$\mathrm{s}=\mathrm{nf}-\mathrm{r}+1 ;$ \%Inversão da ordem dos segmentos realizada pelo espectrômetro

if $(B(s, 1)<\theta)$

$B(s, 1)=-\operatorname{round}(B(s, 1) * 1023 / \max f 1) ;$

$B(s, 2)=B(s, 2)+p i ;$

else

$B(s, 1)=\operatorname{round}(B(s, 1) * 1023 / \max f 1) ;$

end

$\mathrm{B}(\mathrm{s}, 2)=\bmod (\mathrm{B}(\mathrm{s}, 2) * 180 / \mathrm{pi}, 360)$;

fprintf(out, ' $\left.\$ d t=\operatorname{trunc}(0.5+(\$ p w b * \%)) \backslash n ', B(s, 3) /\left(1.0 e^{*} * p^{*} * D t s\right)\right)$;

fprintf(out, '\$pwa $=\$ p w a+0.3 * \$ d t \backslash n '$ );

fprintf(out, 'write(',file', , ',/export/home/CQ/vnmrsys/shapelib/\%s.RF', , ',\%.2f \%.1f \%s. ', ,\$dt) \n', fn, $B(s, 2), B(s, 1))$;

end

fprintf(out, '`n');

fprintf(out, 'input(',Pulse position(5 to 7): ',):\$pos \n\n');

fprintf(out, '\$tpwrma= \%f*\$wq/alpha \n\n', $\operatorname{maxf1/wq)}$;

fprintf(out, 'if $\$$ pos=5 then $\backslash \mathrm{n}^{\prime}$ );

fprintf(out, ' $\quad \mathrm{pw} 5=\$ \mathrm{pwa} \backslash \mathrm{n}$ ') ;

fprintf(out, , tpwrm5 = \$tpwrma $\backslash n^{\prime}$ ) ;

fprintf(out, ' $\quad \operatorname{tpwr} 5=60 \backslash \mathrm{n}^{\prime}$ );

fprintf(out, ' pwpat5=' '\%s' '\n', fn);

fprintf(out, 'endif $\backslash n$ ');

fprintf(out, ' $\backslash \mathrm{n}$ ') ;

fprintf(out, 'if $\$ p o s=6$ then $\backslash n$ ');

fprintf(out, ' $\quad$ ww6= \$pwa $\backslash n^{\prime}$ ) ;

fprintf(out, , tpwrm6= $\$$ tpwrma $\backslash n^{\prime}$ ) ;

fprintf(out, ' tpwr6= 60\n');

fprintf(out, ' pwpat6="'\%s', $\backslash \mathrm{n}$ ', fn); 


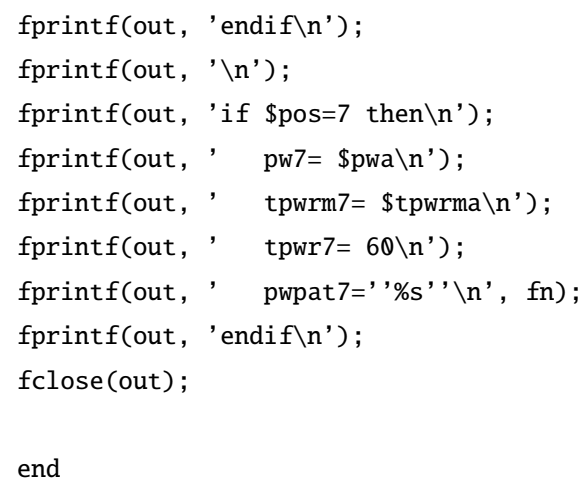




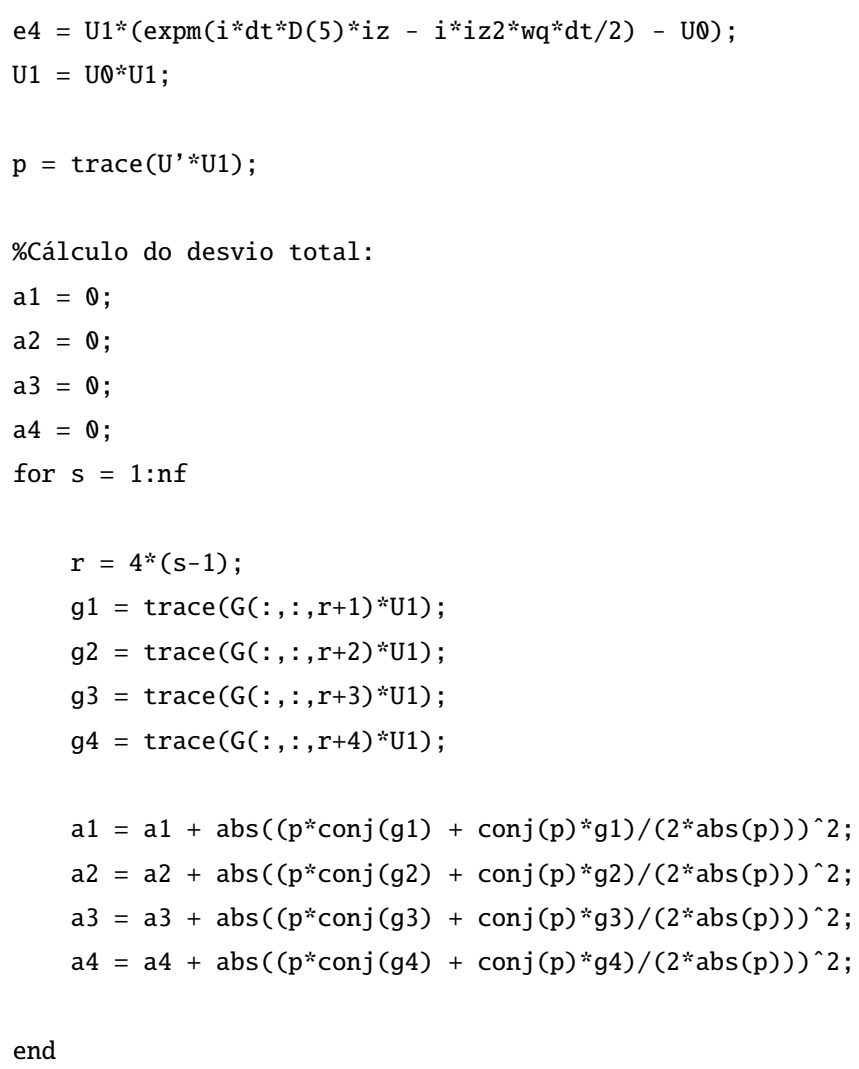

\section{A.3 Exemplo de macro utilizada para criação de porta lógica}

A macro a seguir é escrita na linguagem MAGICAL [59] e serve para calibrar e gerar o arquivo de pulso .RF que contém os parâmetros do pulso modulado. Essa macro é gerada através do código do apêndice A.2. Nesse exemplo, o pulso modulado corresponde a uma porta lógica $\mathrm{CNOT}_{A}$ em um spin 3/2. 
"Total time $(16 \mathrm{kHz})=161.69$ us"

"15 segments"

"w1*t dev. $=0.004779 "$

"phi dev. = 0.007724"

" $(\mathrm{wq} / 2) * \mathrm{t}$ dev. $=0.005836 "$

"w0*t dev. $=0.000629 "$

av

$\mathrm{cr}=\mathrm{f} 1$

$\mathrm{nl}$

nl

$\mathrm{nl}$

$\mathrm{nl}$

$\mathrm{nl}$

$\mathrm{f} 1=\mathrm{cr}$

$\mathrm{cr}=\mathrm{f} 2$

$\mathrm{nl}$

nl

$\mathrm{nl}$

$\mathrm{nl}$

$\mathrm{nl}$

$\mathrm{f} 2=\mathrm{cr}$

movetof

$\mathrm{cr}=\mathrm{f3}$

nl

$\mathrm{nl}$

nl

nl

$\mathrm{nl}$

$\mathrm{f} 3=\mathrm{cr}$

$\mathrm{ph}$

$\$ w q=2.0 * 3.14159265 *(f 3-f 1) / 2.0$

$\$ \mathrm{pwb}=16.255332 \mathrm{e} 6 / \$ \mathrm{wq}$

$\$ \mathrm{pwa}=0.0$

write ('reset' , '/export/home/CQ/vnmrsys/shapelib/cna.RF')

$\$ d t=\operatorname{trunc}(0.5+(\$ p w b * 0.189135))$

$\$ \mathrm{pwa}=\$ \mathrm{pwa}+0.3 * \$ \mathrm{dt}$

write('file', '/export/home/CQ/vnmrsys/shapelib/cna.RF', '319.0 505.0 \%s. ', \$dt)

$\$ \mathrm{dt}=\operatorname{trunc}(0.5+(\$ \mathrm{pwb} * 0.412299))$

$\$$ pwa $=\$$ pwa $+0.3 * \$ \mathrm{dt}$

write('file', '/export/home/CQ/vnmrsys/shapelib/cna.RF', '131.0 117.0 \%s.' , \$dt)

$\$ \mathrm{dt}=\operatorname{trunc}(0.5+(\$ \mathrm{pwb} * 0.131424))$

$\$$ pwa $=\$$ pwa $+0.3 * \$ d t$

write('file', '/export/home/CQ/vnmrsys/shapelib/cna.RF', '98.5 1023.0 \%s.', \$dt)

$\$ \mathrm{dt}=\operatorname{trunc}(0.5+(\$ \mathrm{pwb} * 0.355335))$

$\$$ pwa $=\$$ pwa $+0.3 * \$ d t$ 


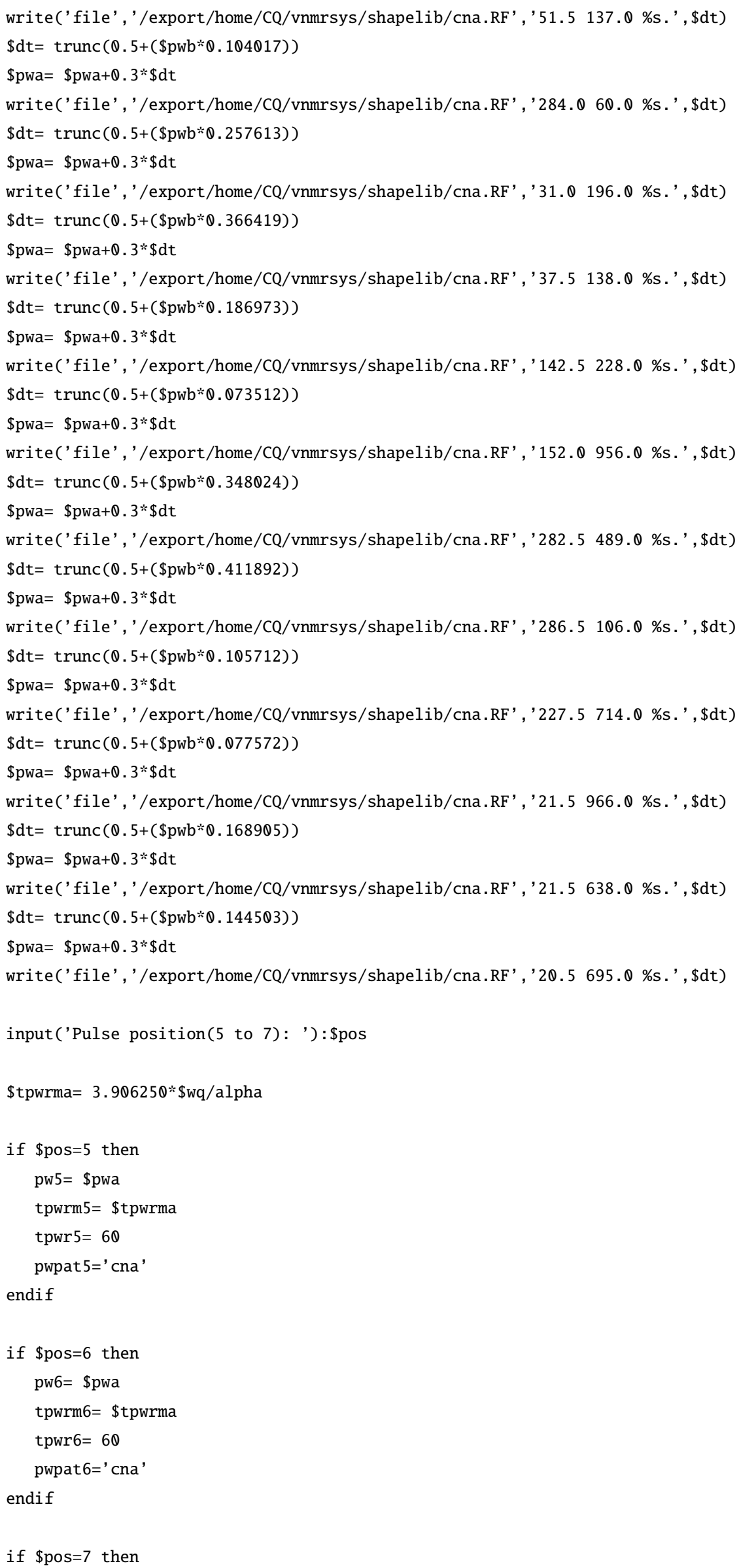


$\mathrm{pw} 7=\$ \mathrm{pwa}$

tpwrm7= \$tpwrma

$\operatorname{tpwr} 7=60$

pwpat7='cna'

endif

\section{A.4 Código da sequiência de pulsos utilizada para realizar os experimentos de CQ}

O código a seguir é utilizado para produzir a seqüência de pulsos gerada pelo espectrômetro. Ele é escrito basicamente em linguagem C. A principal diferença com relação a um código C padrão está nas operações e variáveis chamadas de tempo real, as quais produzem modificações na sequiência ao longo das diferentes médias de uma mesma aquisição. Essas variáveis possuem obrigatoriamente a sintaxe vi em que $i$ é um número inteiro entre 1 e 14 . Descrições de comandos específicos de programação podem ser encontrados em [59]. Um exemplo de seqüência usando média espacial via gradientes de campo é apresentada ao final deste apêndice.

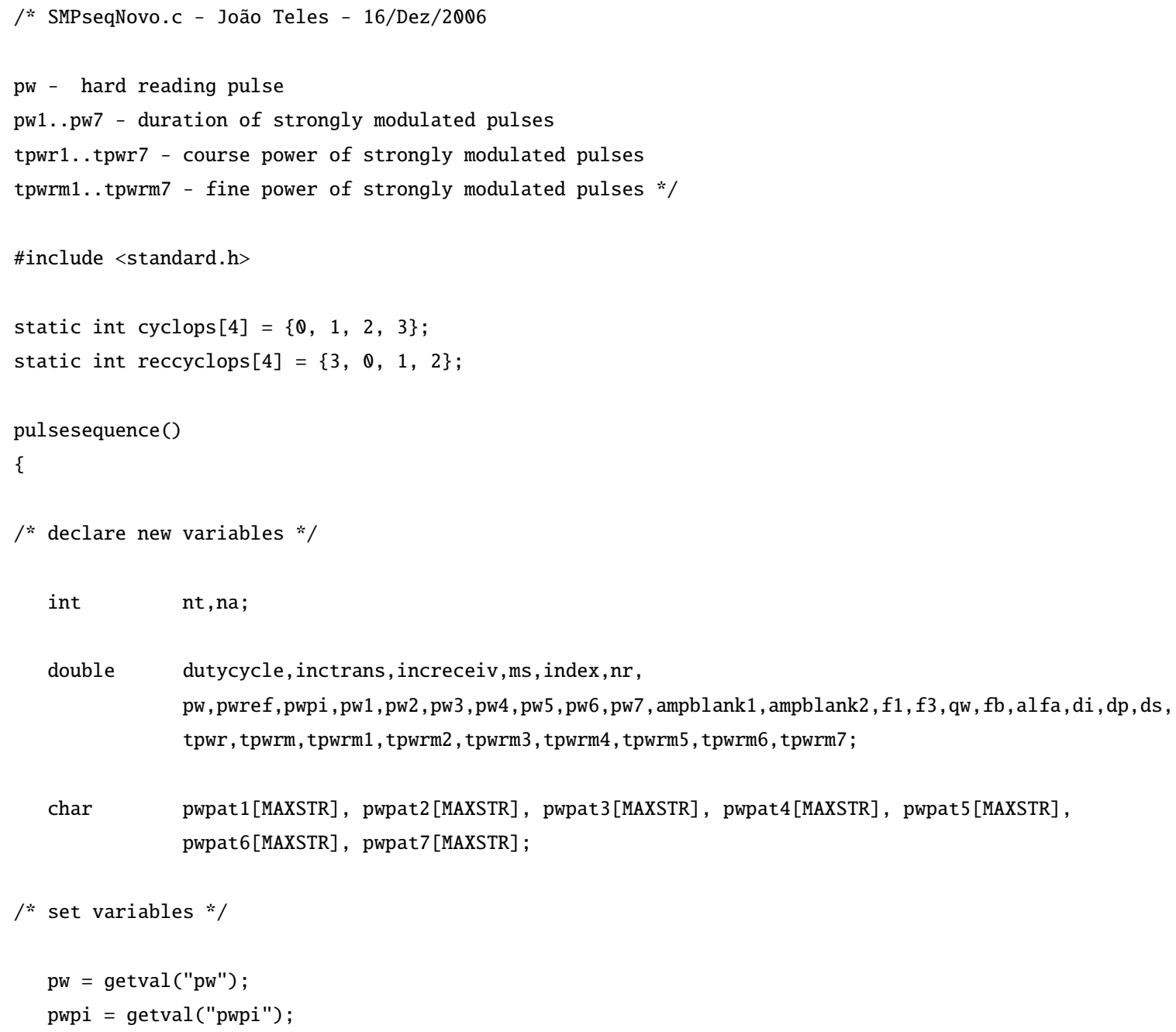




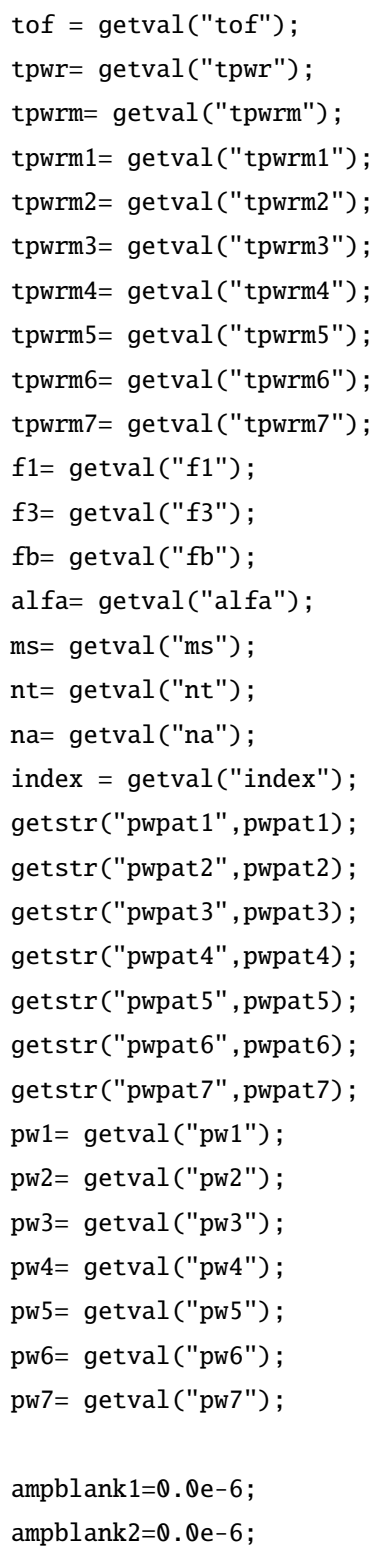




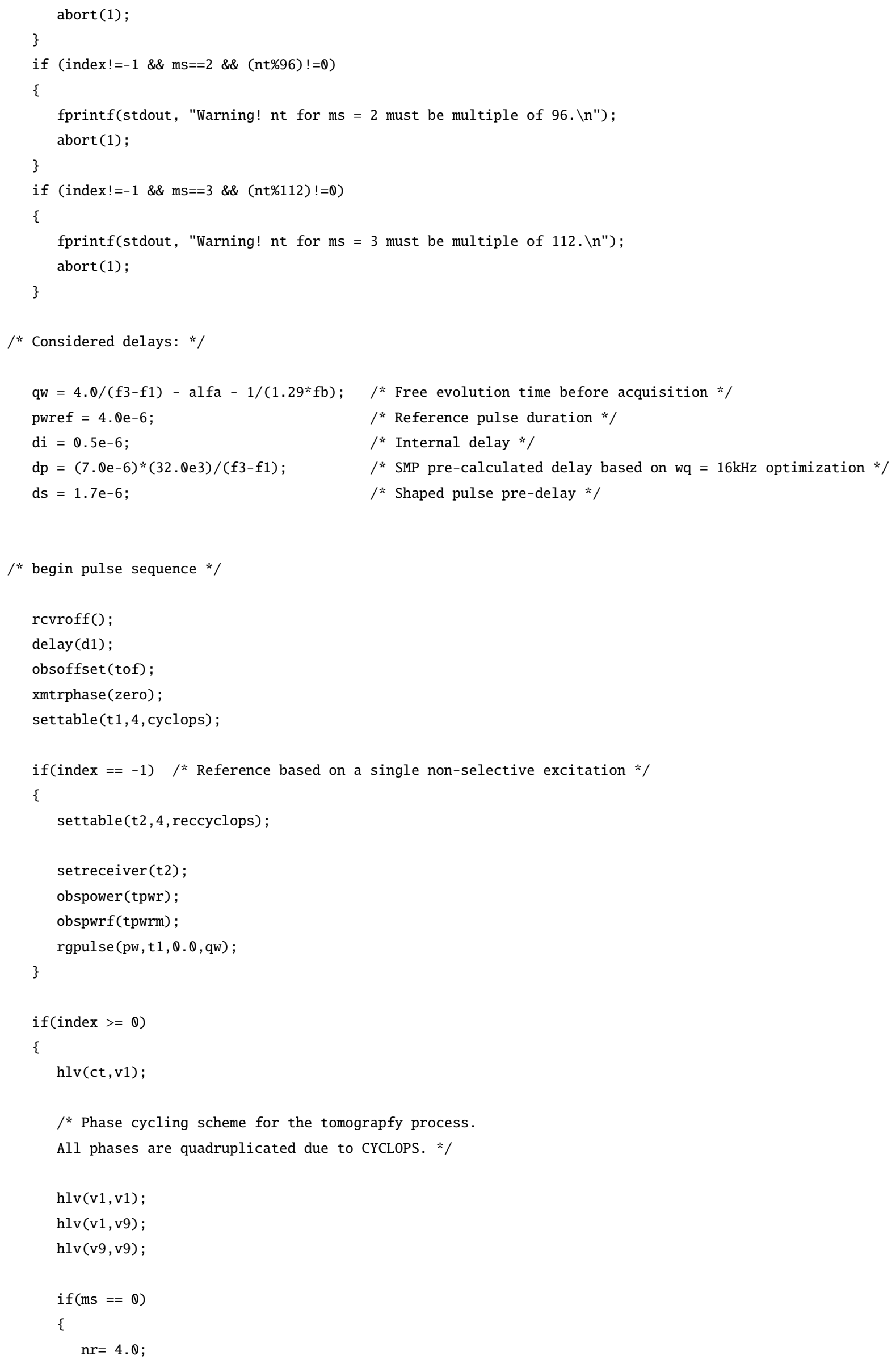




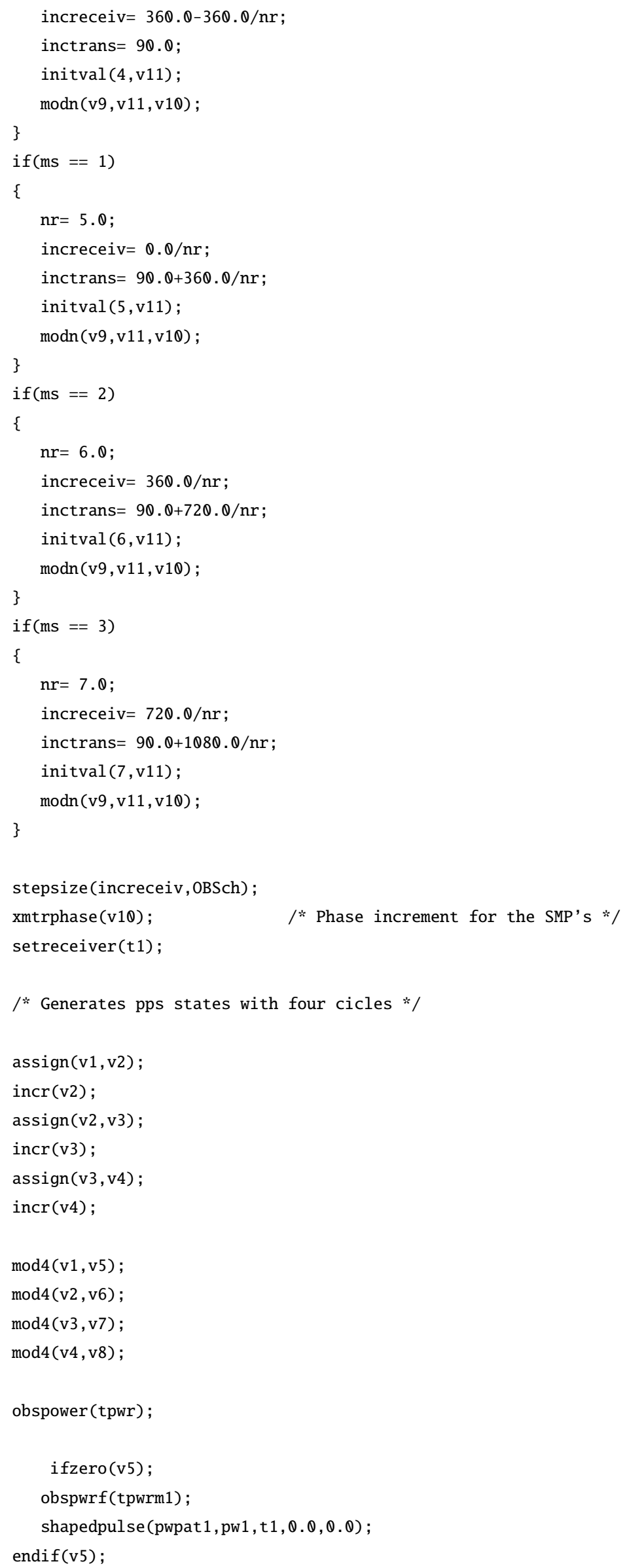




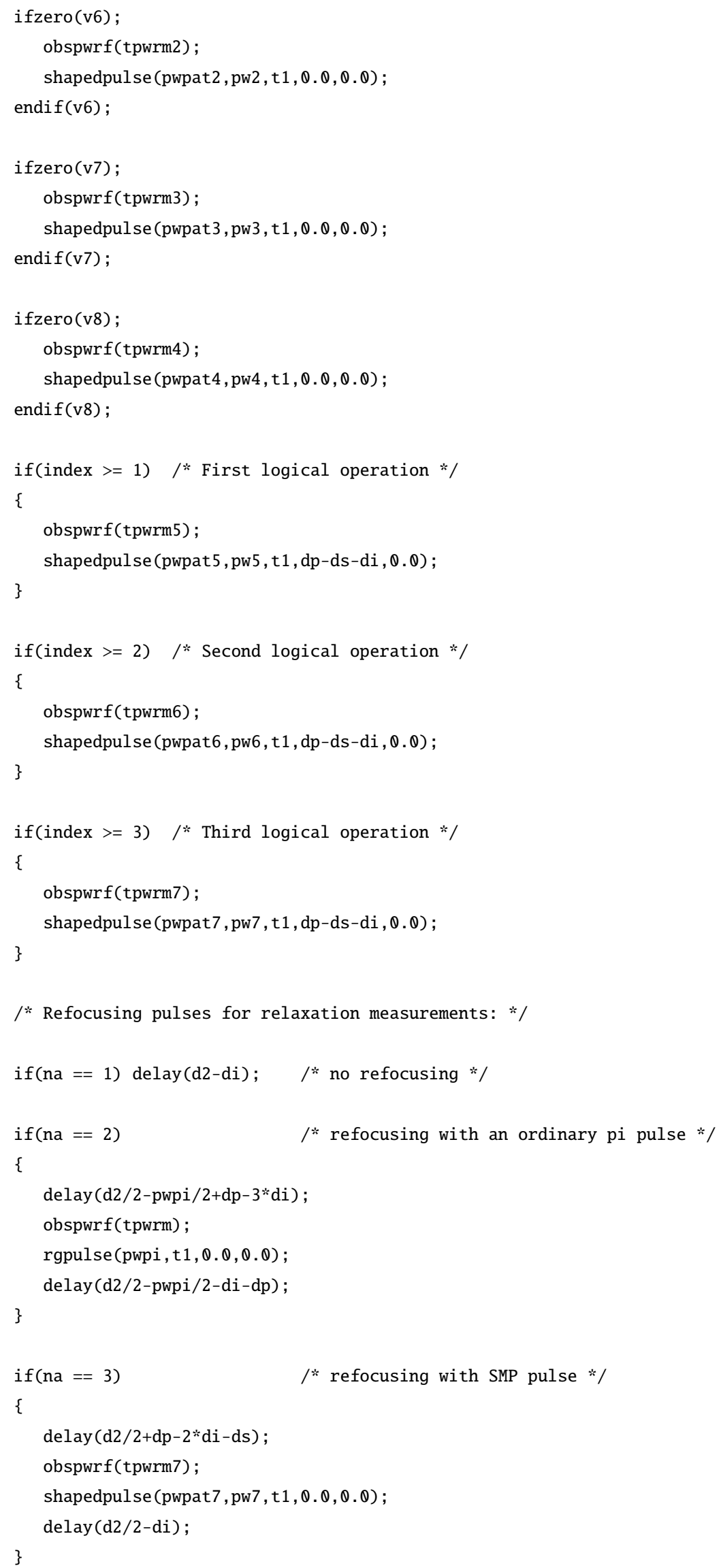




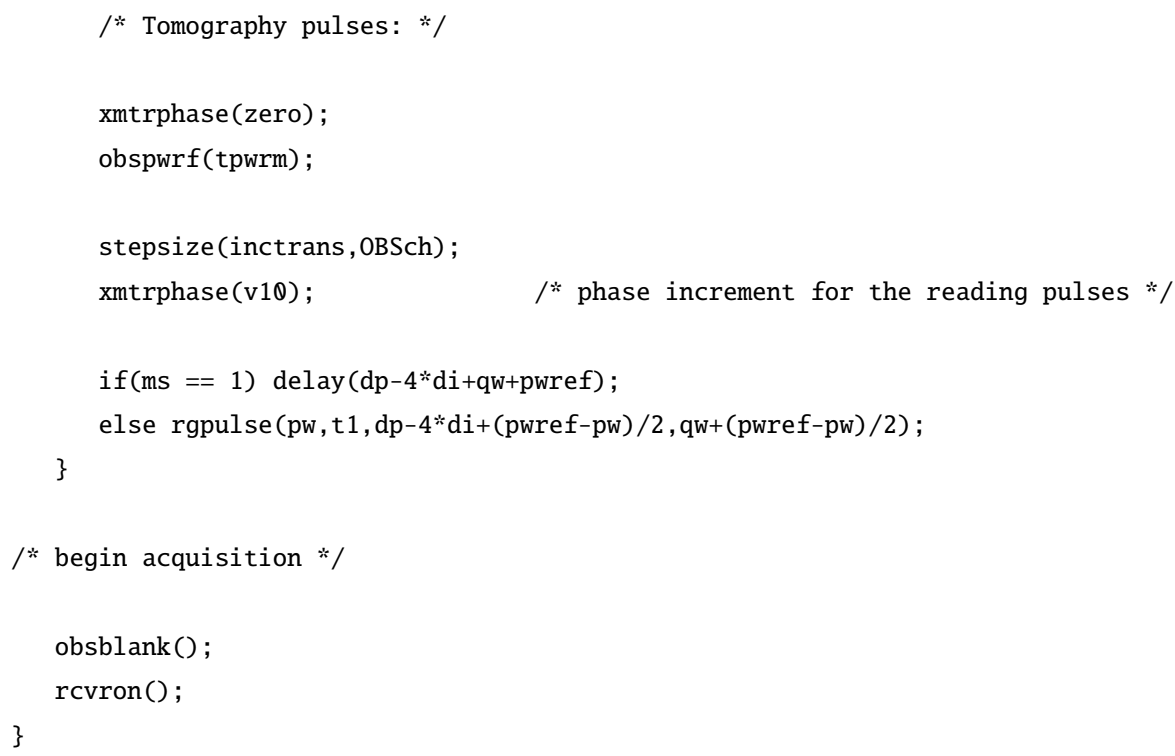

Seqüência de pulsos utilizando gradientes ao invés de média temporal:

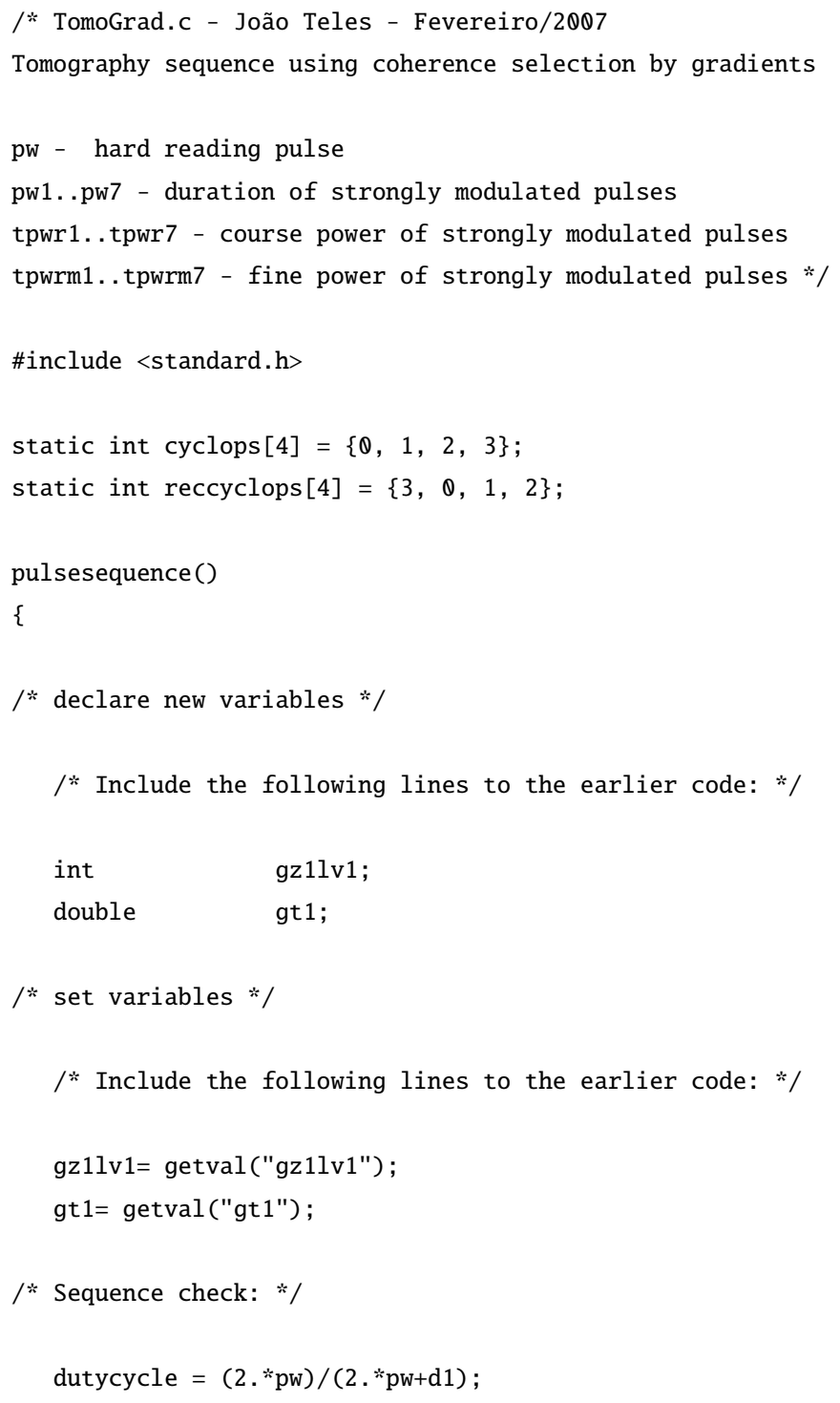




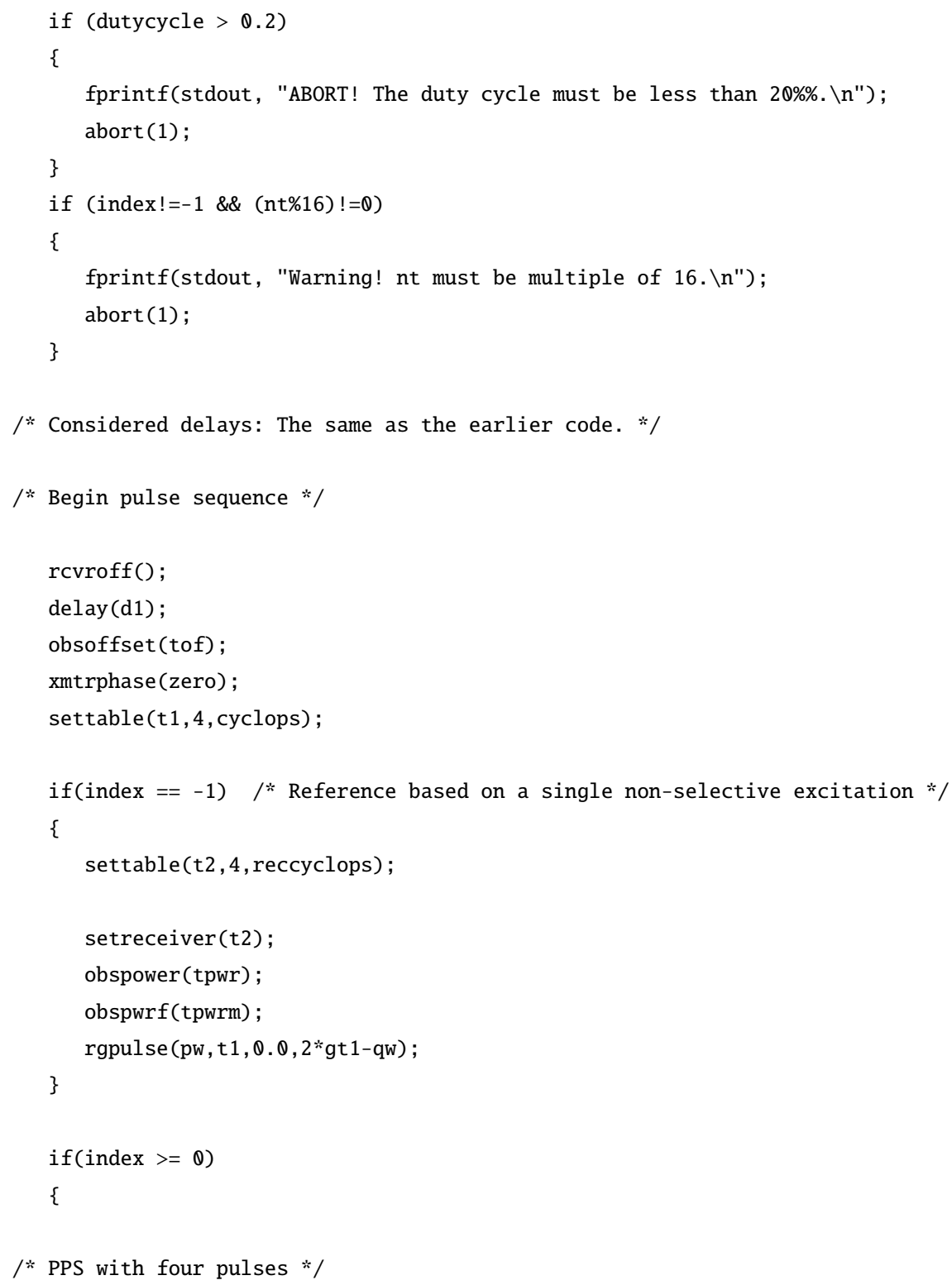




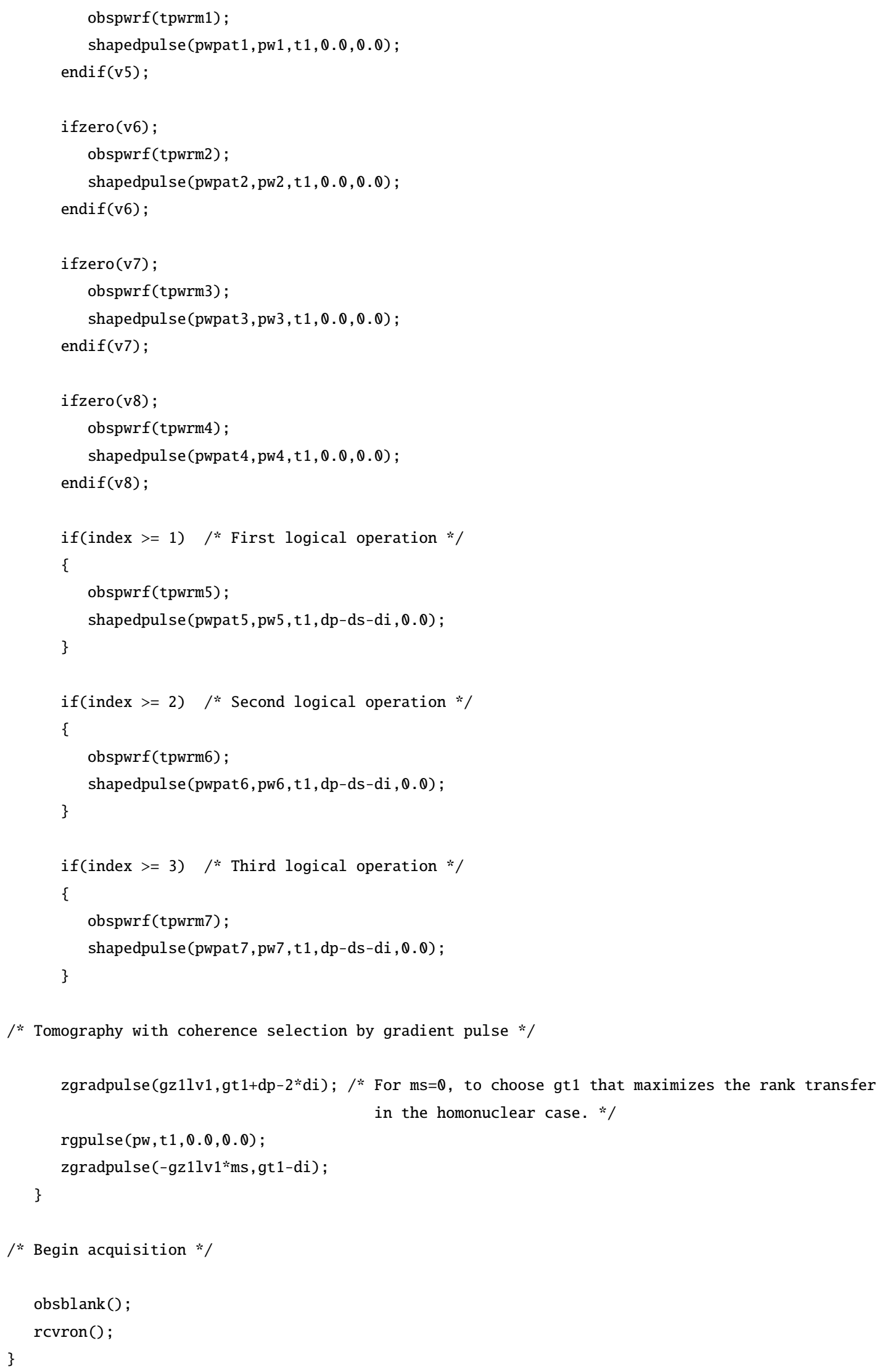




\section{A.5 Código para reconstrução da matriz densidade a partir dos experimentos de TEQ}

O código a seguir foi escrito em linguagem C e possibilita a reconstrução da matriz densidade a partir dos dados experimentais obtidos pelo método de TEQ. O sistema de equações obtido da aplicação do método dos mínimos quadrados na equação 3.49 encontra-se na rotina Sistema incluída no código abaixo. Para não deixar a listagem demasiadamente longa, foi incluído somente o caso do spin 3/2. Esse sistema foi obtido a partir de um código escrito em Maple que está listado logo após o código em C. Esse código é bastante geral, podendo ser usado para obter o sistema de um spin $7 / 2$ ou maior.

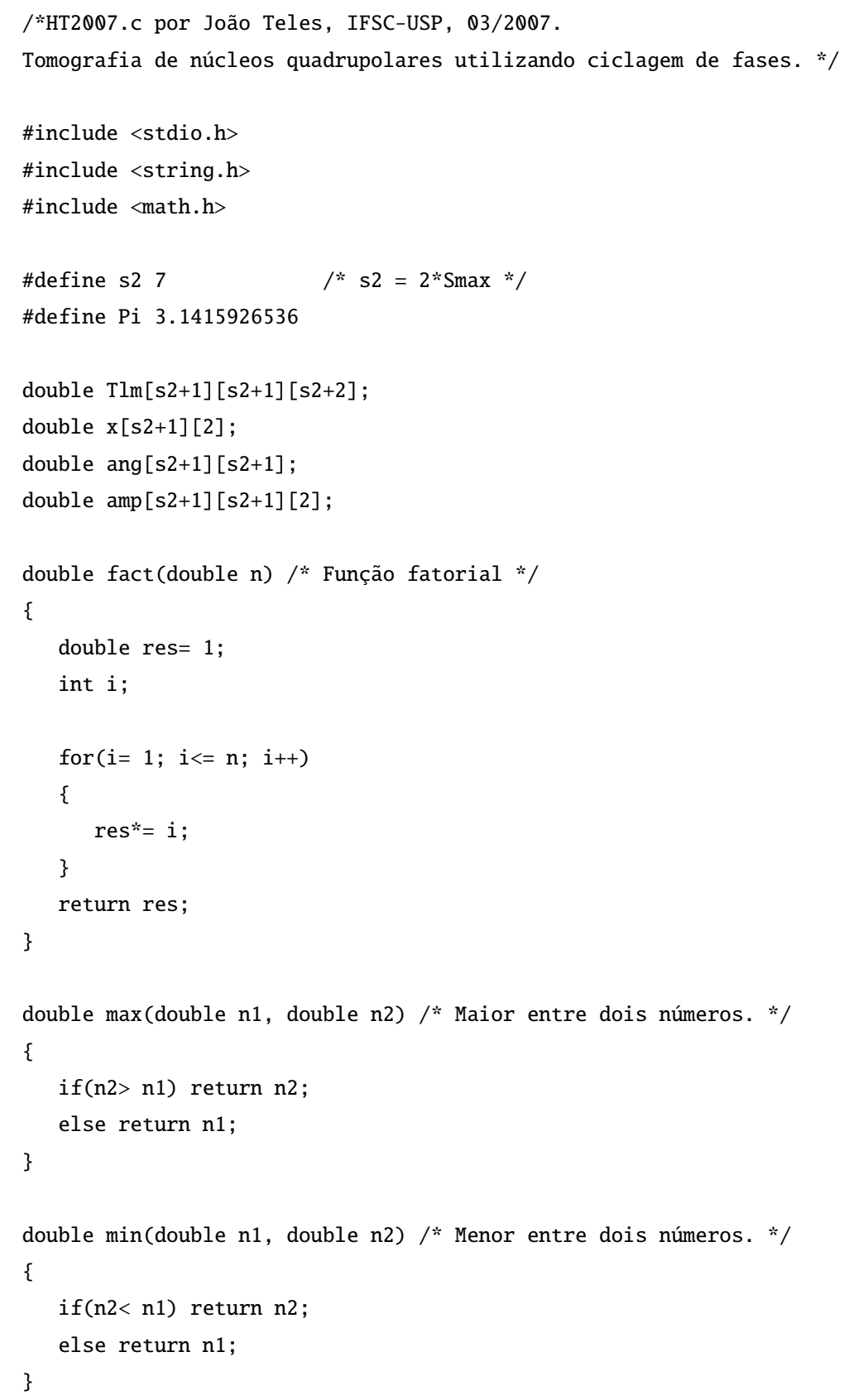


double sgn(double $\mathrm{n} 1$, double n2) /* Retorna o sinal do maior em módulo entre dois números. */

\{

$\operatorname{if}(\max (\mathrm{n} 2, \mathrm{n} 1)>=-\min (\mathrm{n} 2, \mathrm{n} 1))$ return 1 ;

else return -1 ;

\}

void Criar_Tlm(double s) /* Cria tensores irredutíveis (s <= Smax).

São calculados apenas os elementos de matriz não nulos

e somente os tensores com $\mathrm{m}>=0$. */

\{

int $1, \mathrm{~m}, \mathrm{i}$;

double cl, cg1, cg2, z;

double ag, bg, cg, zmin, zmax;

double $f 1, f 2, f 3, f 4, f 5$;

for $(1=0 ; 1<=2 * s ; 1++) \quad / *$ Varredura do rank. */

\{

$\mathrm{cl}=\operatorname{fact}(1) * \operatorname{sqrt}((2 * \mathrm{~s}+1) * \operatorname{fact}(2 * \mathrm{~s}-1) / \operatorname{fact}(2 * \mathrm{~s}+1+1))$;

for $(\mathrm{m}=0 ; \mathrm{m}<=1 ; \mathrm{m}++) \quad / *$ Varredura da ordem. */

\{

for $(i=1 ; i<=2 * s-m+1 ; i++)$

\{

$\mathrm{ag}=\mathrm{s}-\mathrm{i}-\mathrm{m}+1$

$\mathrm{bg}=\mathrm{m}$;

$\mathrm{cg}=\mathrm{s}-\mathrm{i}+1$;

$\operatorname{cg} 1=\theta$;

$\mathrm{zmin}=\max (\theta, 1+\mathrm{cg}-\mathrm{s}) ;$

$\mathrm{zmax}=\min (1, \mathrm{~s}+\mathrm{cg}) ;$

$\mathrm{zmax}=\min (\mathrm{zmax}, \mathrm{s}+\mathrm{l}+\mathrm{ag}) ;$

for $(z=z \min ; z<=\operatorname{zmax} ; z++)$

\{

$\mathrm{f} 1=\mathrm{fact}(\mathrm{s}+\mathrm{l}+\mathrm{ag}-\mathrm{z})$

$\mathrm{f} 2=\operatorname{fact}(\mathrm{s}-\mathrm{ag}+\mathrm{z})$;

$\mathrm{f3}=\operatorname{fact}(1-\mathrm{z})$;

$\mathrm{f} 4=\operatorname{fact}(\mathrm{s}+\mathrm{cg}-\mathrm{z})$;

$\mathrm{f} 5=\operatorname{fact}(\mathrm{s}-1-\mathrm{cg}+\mathrm{z})$;

$\operatorname{cg} 1+=\operatorname{pow}(-1,1+m+z) * f 1 * \mathrm{f} 2 /(\operatorname{fact}(z) * \mathrm{f} 3 * \mathrm{f} 4 * \mathrm{f} 5) ;$

\}

$\operatorname{cg} 2=\operatorname{sqrt}($ fact $(\mathrm{s}-\mathrm{cg}) *$ fact $(\mathrm{s}+\mathrm{cg}) /($ fact $(\mathrm{s}-\mathrm{ag}) *$ fact $(\mathrm{s}+\mathrm{ag}) * \operatorname{fact}(1-\mathrm{bg}) *$ fact $(1+\mathrm{bg})))$;

$\operatorname{Tlm}[1][\mathrm{m}][\mathrm{i}]=\operatorname{sqrt}((2 * 1+1) /(2 * \mathrm{~s}+1)) * \mathrm{cl} * \operatorname{cg} 1 * \operatorname{cg} 2$;

\}

\}

\}

double wigner(double 1 , double $\mathrm{m}$, double th) $/ *$ Calcula as funções de Wigner $\left.\mathrm{d}^{\wedge}\{1\}\right\}_{-}\{1, \mathrm{~m}\}(-$ th) $* /$

\{

double k, kmax, v1, vs, fc, fs;

$\mathrm{vs}=0$;

if $(m>=0)$

\{

$\mathrm{m}=\operatorname{abs}(\mathrm{m}) ;$ 


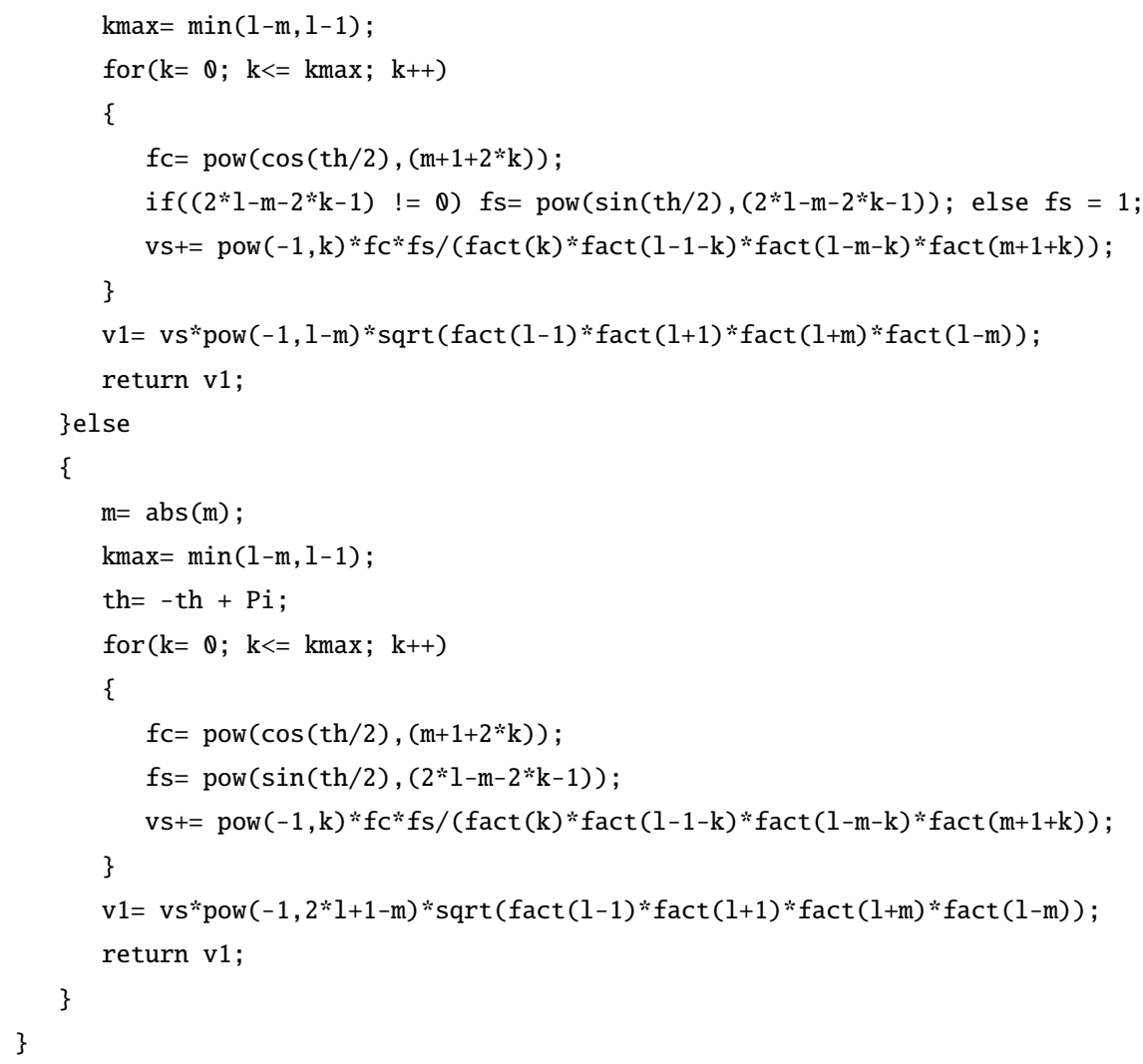




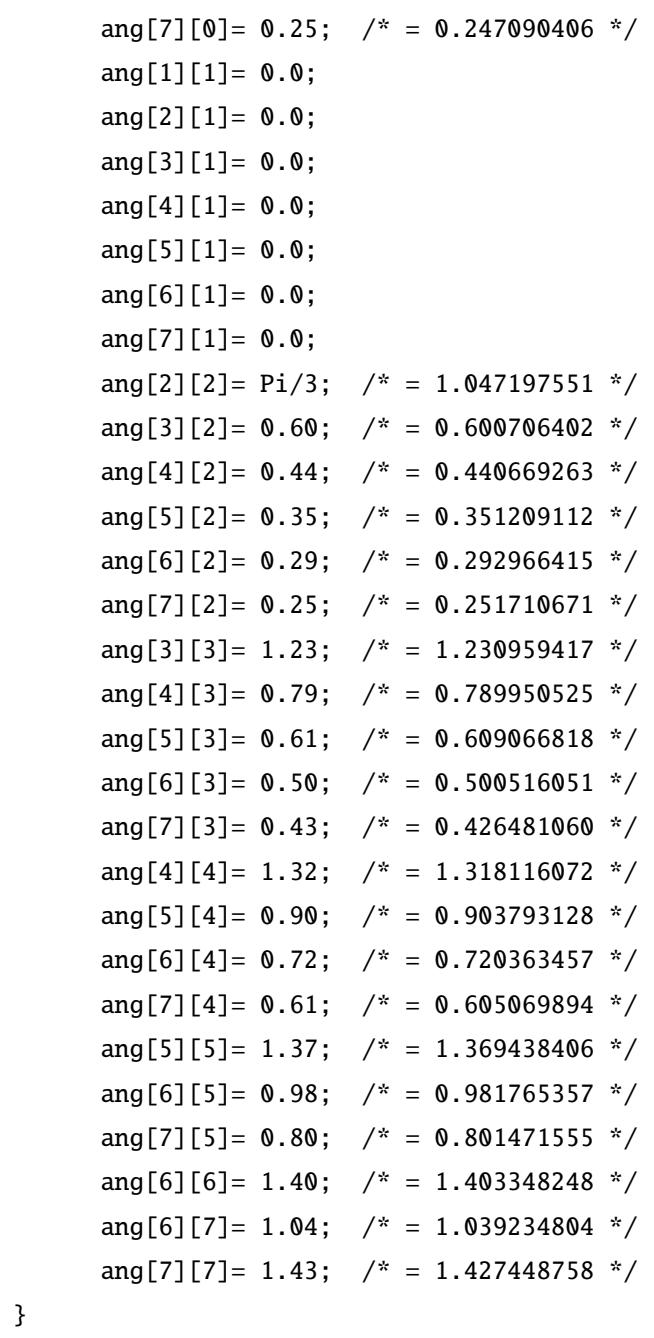




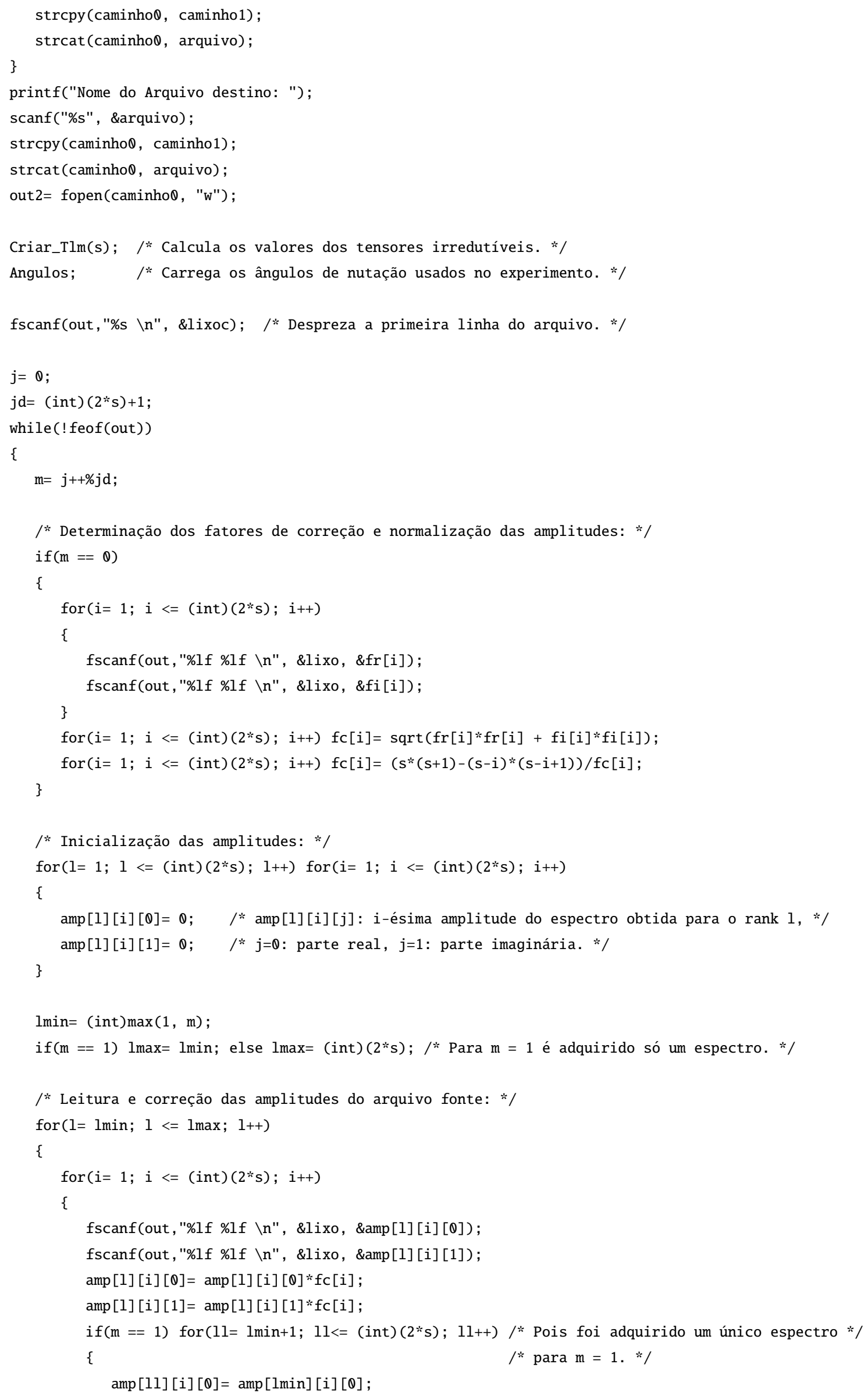




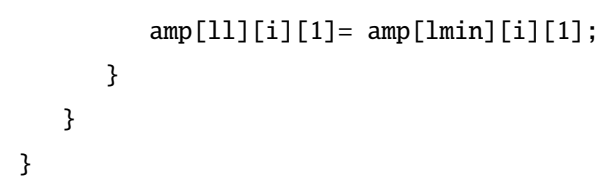




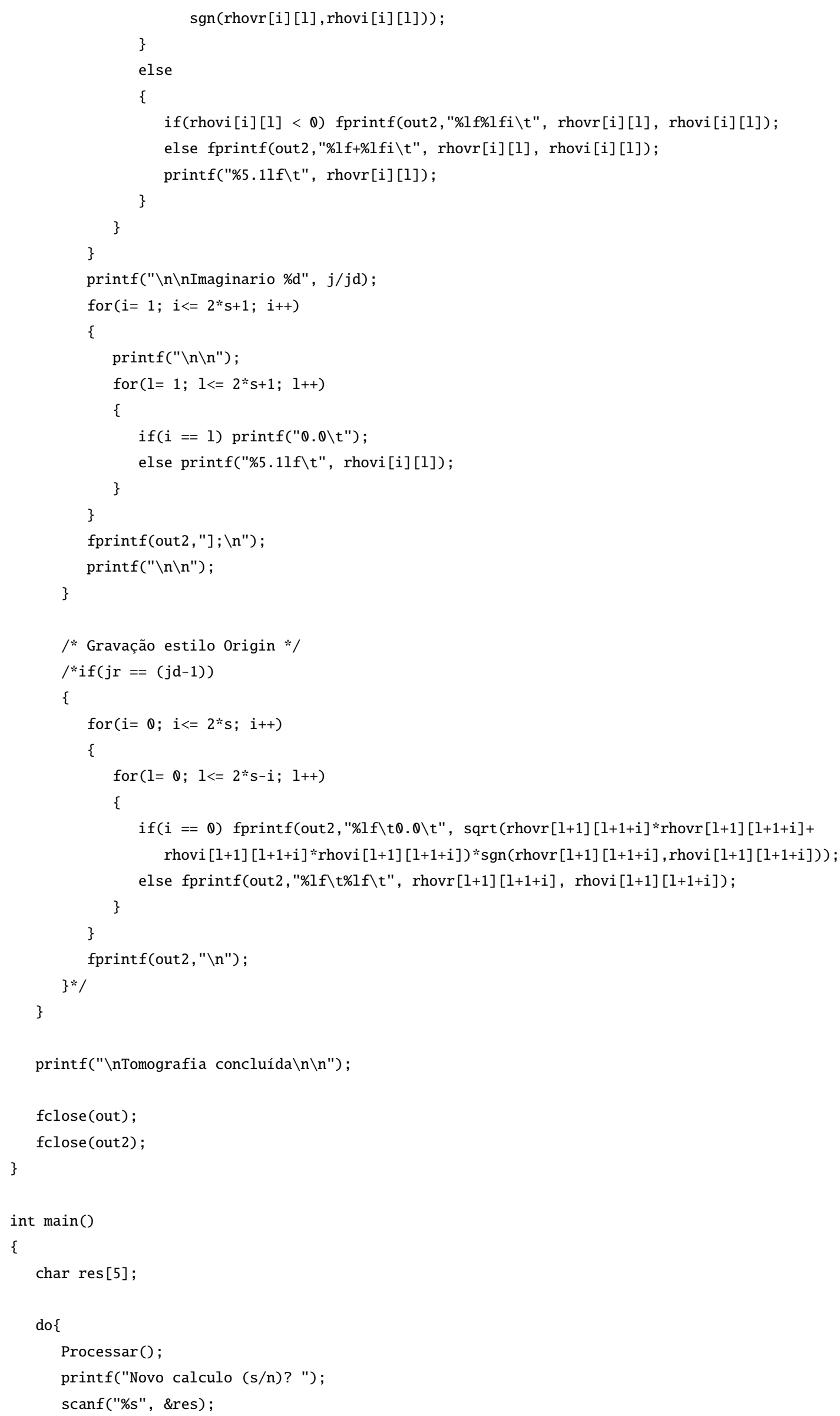




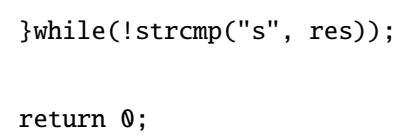

Código em Maple para gerar o sistema de equações:

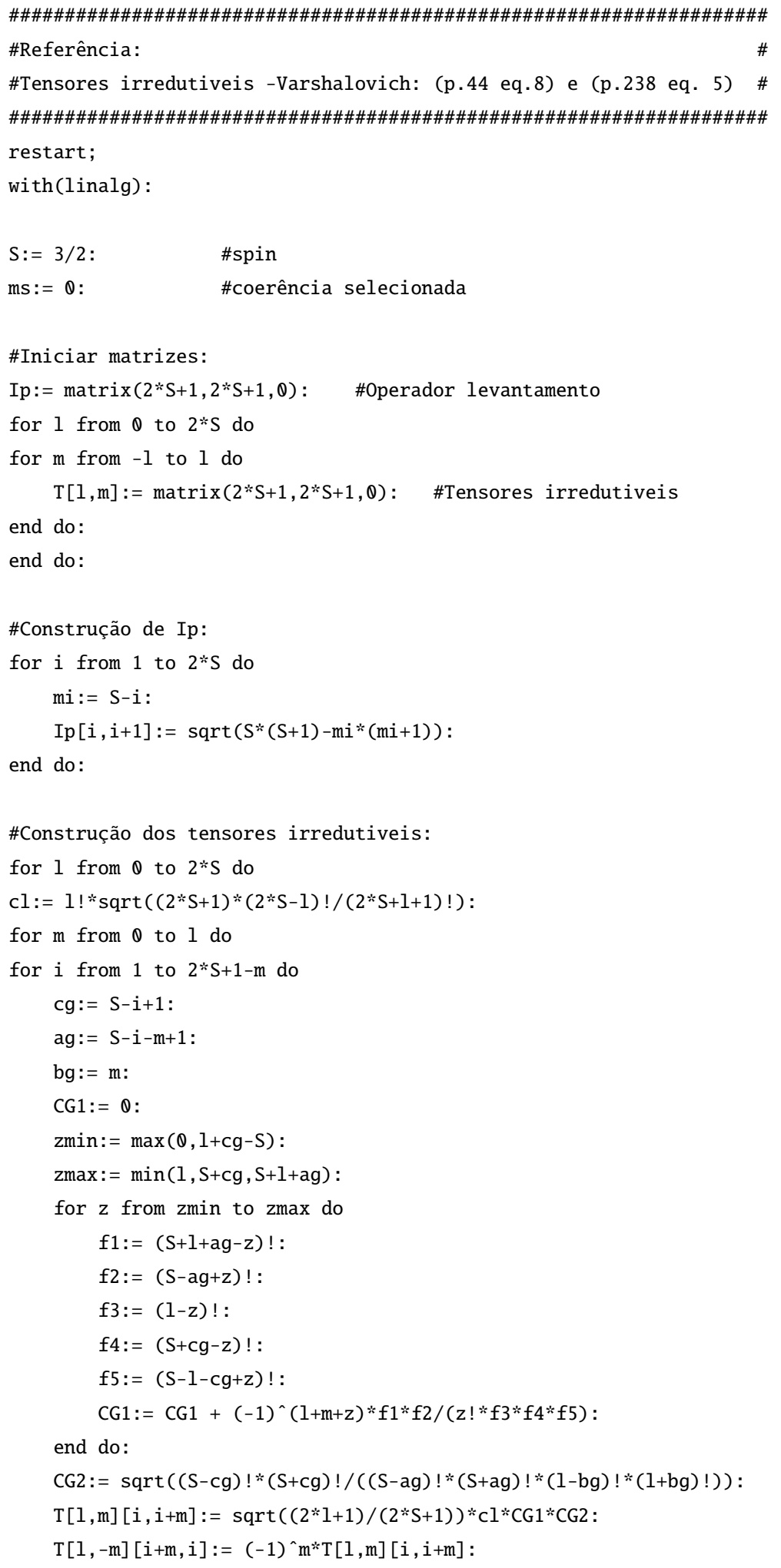




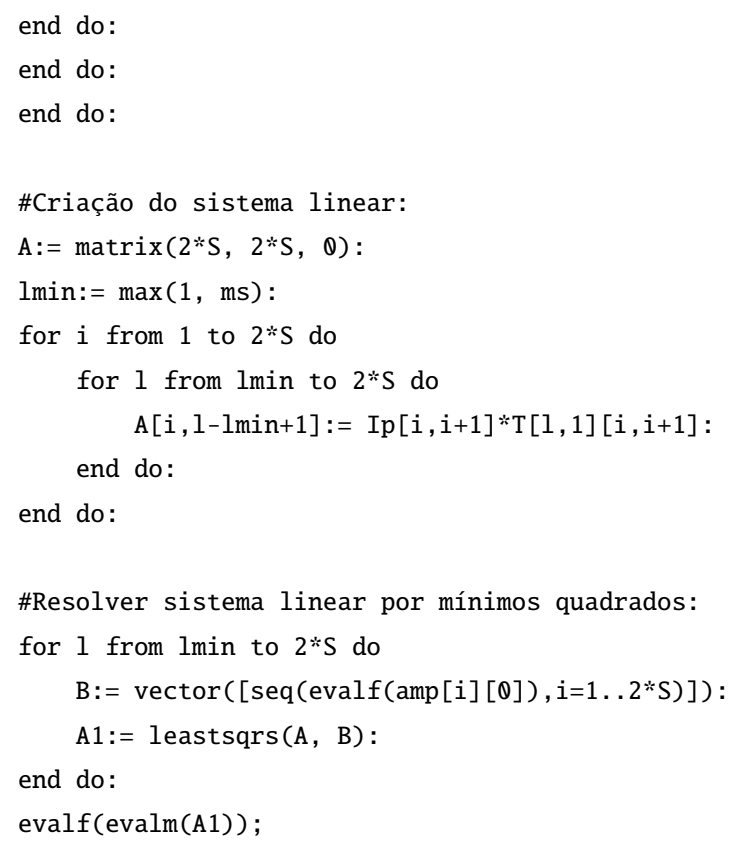

\section{A.6 Macros para programar e ler a seqüência de experimen- tos usados no processo de TEQ}

A primeira macro a seguir é usada para programar a seqüência de experimentos cujos resultados são fornecidos para o código de reconstrução listado em A.5. A variável \$v determina o número de repetições do bloco nt correspondente a cada coerência. A variável \$p determina até que etapa o algoritmo quântico será realizado. O parâmetro d2 controla o tempo de evolução livre usado nos estudos de dinâmica e relaxação. A ordem em que a seqüência de parâmetros é implementada é especificada pelo comando array, vide [59]. A segunda macro deste apêndice realiza a leitura das partes reais e imaginárias de cada linha de cada espectro obtido. O resultado é automaticamente gravado no arquivo mark1d.out através do comando mark. Essas macros são específicas para o caso de spin $3 / 2$.

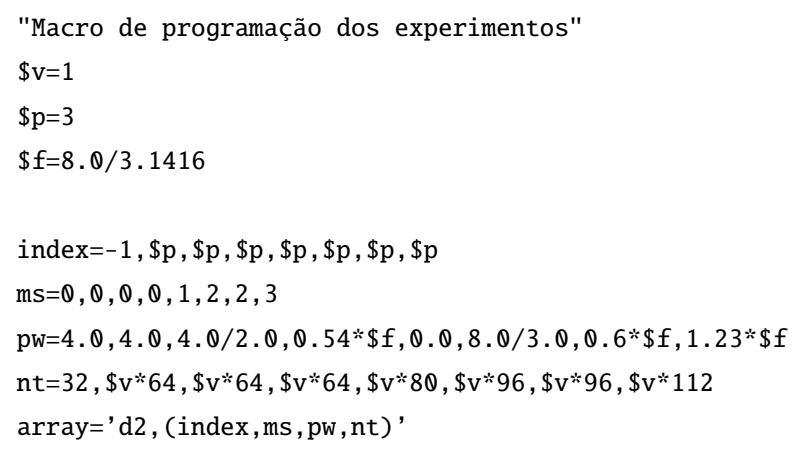


"Macro de leitura do resultado dos experimentos"

ds (1)

ph

aph

mark ('reset')

$\$ \mathrm{x}=1$

repeat

ds $(\$ x)$

av

$\mathrm{cr}=\mathrm{f} 1$

$\mathrm{nl}$

nl

nl

nl

nl

ph

mark

phase $(-90)$

mark

phase (90)

av

$\mathrm{cr}=\mathrm{f} 2$

nl

nl

nl

nl

nl

ph

mark

phase (-90)

mark

phase (90)

av

$\mathrm{cr}=\mathrm{f3}$

nl

nl

nl

nl

nl

ph

mark

phase (-90)

mark

phase (90)

$\$ \mathrm{x}=\$ \mathrm{x}+1$

until $\$ \mathrm{x}=$ arraydim +1

$\$$ 


\section{A.7 Resultados experimentais da TEQ}

Este apêndice contém as matrizes densidade completas obtidas experimentalmente através do emprego do método de TEQ proposto neste trabalho. Esses resultados correspondem ao experimento de criação dos estados de Bell, à implementação do Algoritmo de Deutsch e à implementação do algoritmo de busca para 2 q-bits. Os valores inscritos nos círculos e nos quadrados correspondem, respectivamente, à parte real e à parte imaginária do respectivo elemento. Valores negativos são representados com uma barra sobre os números.

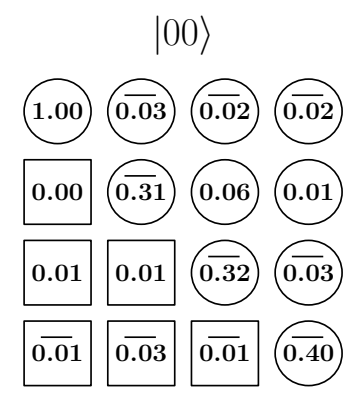

|01〉

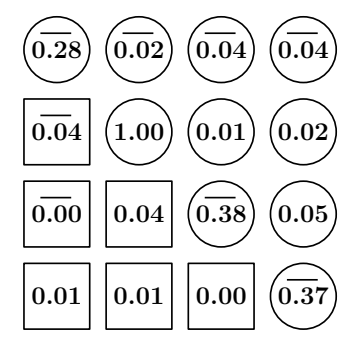

$|10\rangle$

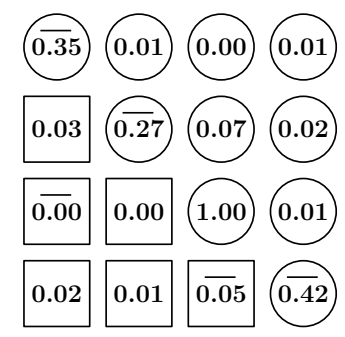

$|11\rangle$

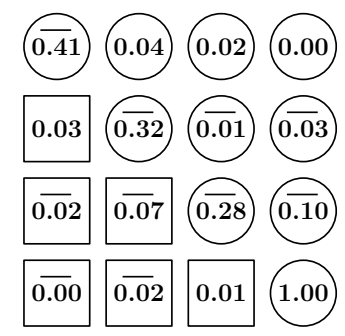

$\mathrm{H}_{A}|00\rangle$

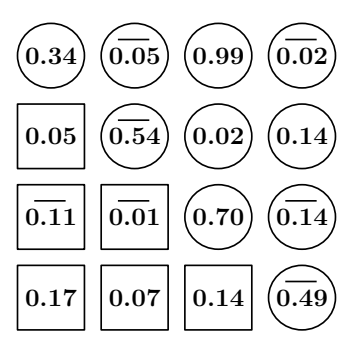

$\mathrm{H}_{A}|01\rangle$

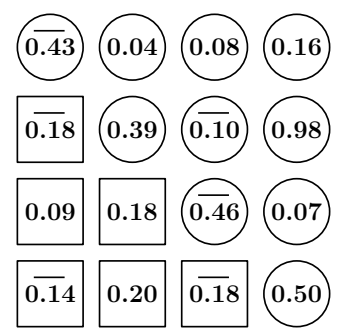

$\mathrm{H}_{A}|10\rangle$

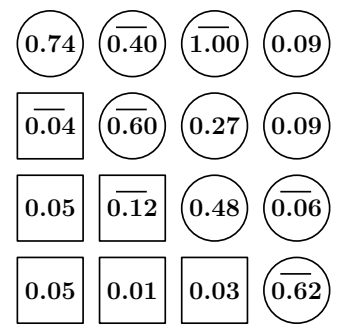

$\mathrm{H}_{A}|11\rangle$

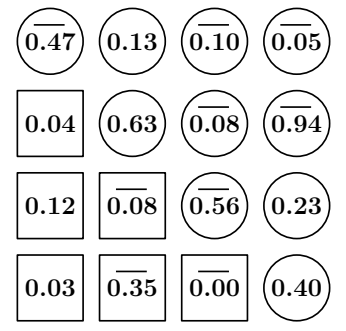

$\mathrm{CN}_{A} \mathrm{H}_{A}|00\rangle$

0.420 .170 .010 .89

0.230 .040 .090 .04

$\begin{array}{lll}0.23 & 0.15 & 0.55 \\ 0.24\end{array}$

\begin{tabular}{llllll}
\hline$\overline{0.45}$ & $\overline{0.34}$ & $\overline{0.04}$ & 0.69 \\
\hline
\end{tabular}

$\mathrm{CN}_{A} \mathrm{H}_{A}|01\rangle$

(0.47) 0.040 .200 .23

$\begin{array}{llll}0.18 & 0.27 & 0.94 & 0.02\end{array}$

\begin{tabular}{llll}
\hline$\overline{0.21}$ & 0.33 & 0.54 & 0.30 \\
\hline
\end{tabular}

\begin{tabular}{lllll}
0.11 & 0.38 & 0.41 & 0.34 \\
\hline
\end{tabular}

$$
\mathrm{CN}_{A} \mathrm{H}_{A}|10\rangle
$$

0.880000000

$\begin{array}{lll}0.24 & 0.56 & 0.01 \\ 0.36\end{array}$

$\begin{array}{llll}0.16 & 0.10 & 0.65 & 0.30\end{array}$

\begin{tabular}{llllll}
\hline 0.12 & $\overline{0.24}$ & 0.11 & 0.37 \\
\hline
\end{tabular}

$\mathrm{CN}_{A} \mathrm{H}_{A}|11\rangle$

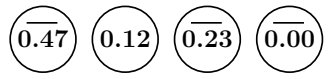

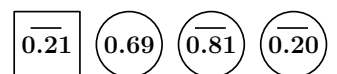

\begin{tabular}{lllll}
0.16 & 0.59 & 0.42 & 0.23 \\
\hline
\end{tabular}

\begin{tabular}{lllll}
\hline 0.23 & 0.05 & 0.10 & 0.68 \\
\hline
\end{tabular}

Figura 53: Resultados experimentais da TEQ relacionados ao experimento de criação de estados de Bell. 


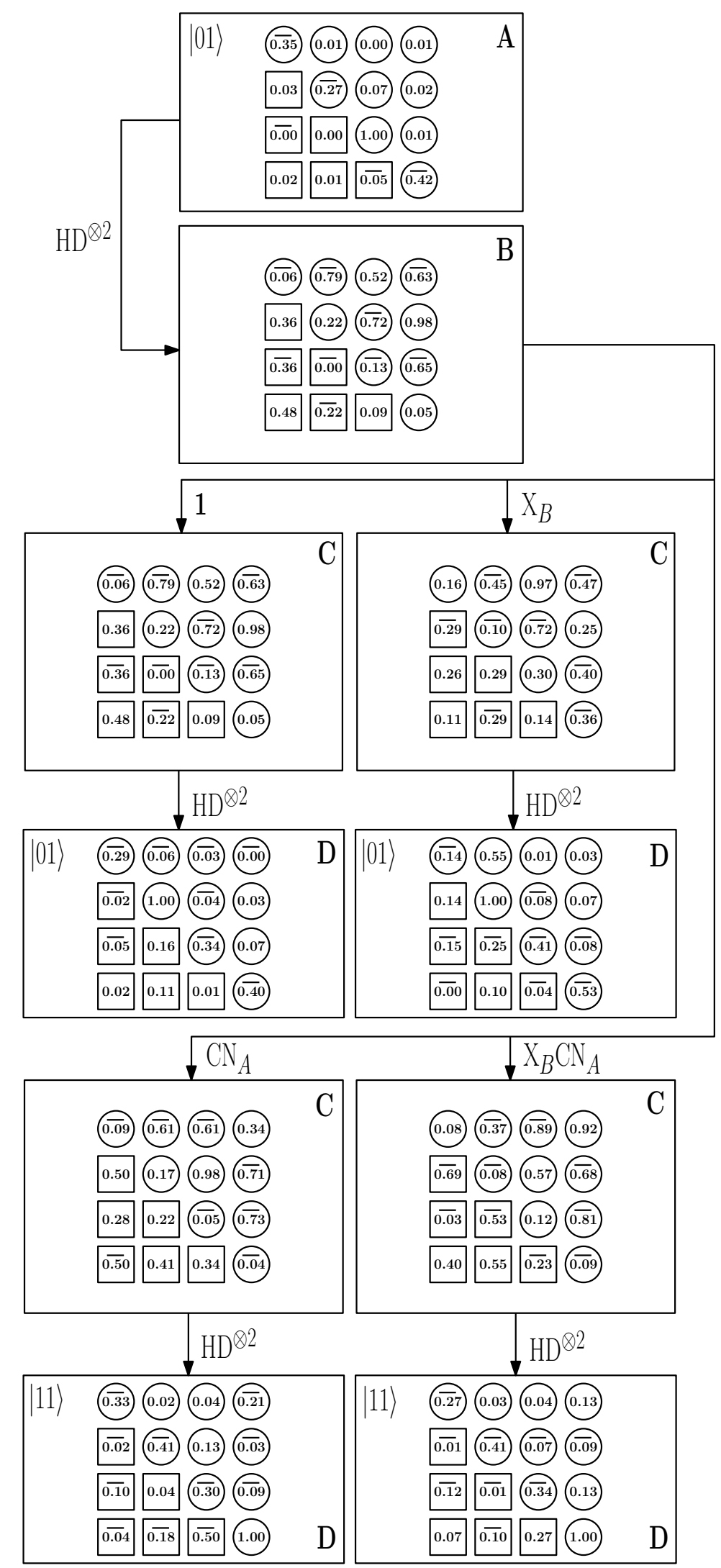

Figura 54: Resultados experimentais da TEQ relacionados à implementação do algoritmo de Deutsch. 


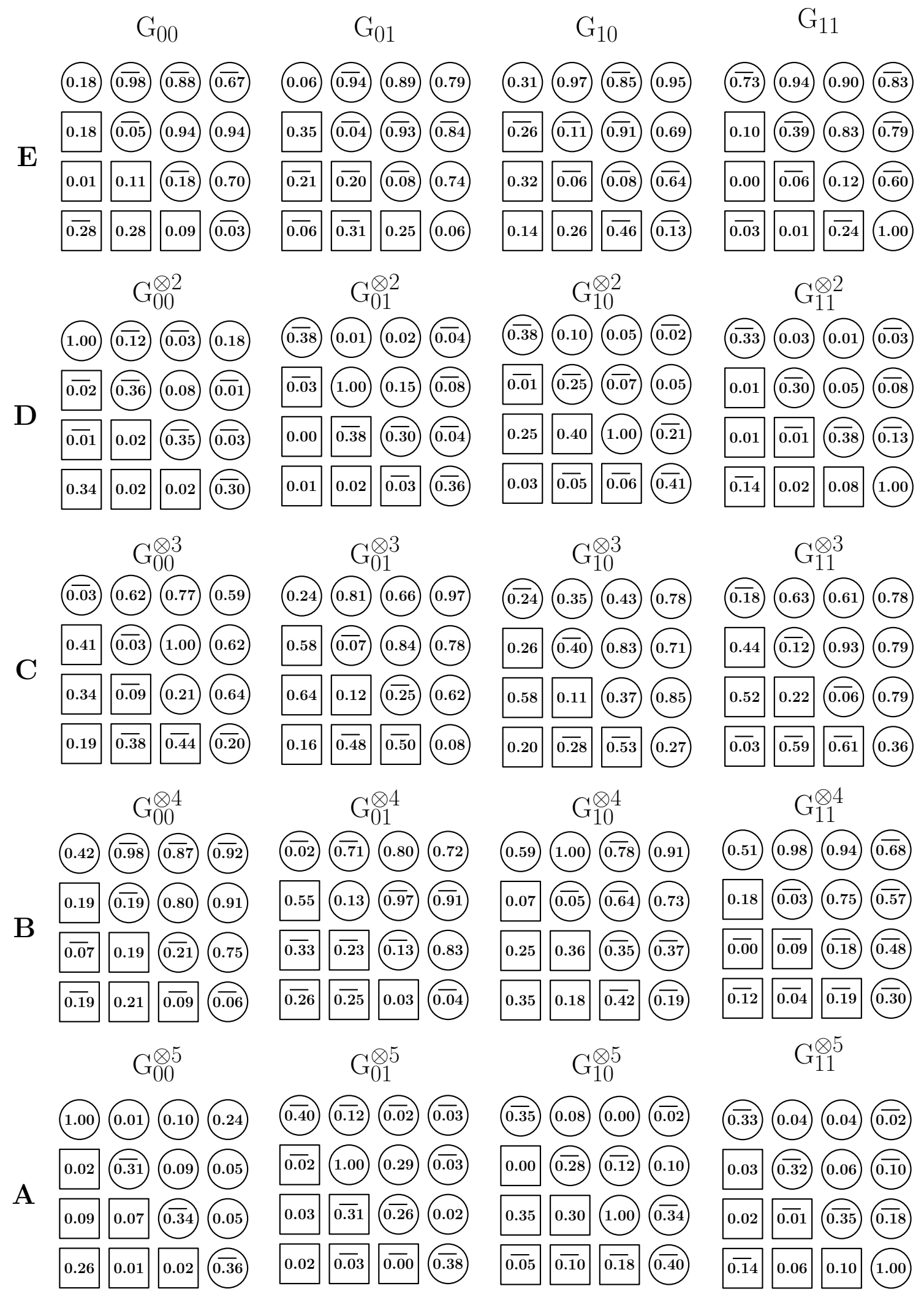

Figura 55: Resultados experimentais da TEQ relacionados à implementação do algoritmo de Grover. 


\section{A.8 Tensores irredutíveis e funções de Wigner para spin 3/2}

$$
\begin{aligned}
& m=0 \\
& T_{1,0}=\frac{1}{2 \sqrt{5}}\left[\begin{array}{rrrr}
3 & 0 & 0 & 0 \\
0 & 1 & 0 & 0 \\
0 & 0 & -1 & 0 \\
0 & 0 & 0 & -3
\end{array}\right] \quad T_{2,0}=\frac{1}{2}\left[\begin{array}{rrrr}
1 & 0 & 0 & 0 \\
0 & -1 & 0 & 0 \\
0 & 0 & -1 & 0 \\
0 & 0 & 0 & 1
\end{array}\right] \quad T_{3,0}=\frac{1}{2 \sqrt{5}}\left[\begin{array}{rrrr}
1 & 0 & 0 & 0 \\
0 & -3 & 0 & 0 \\
0 & 0 & 3 & 0 \\
0 & 0 & 0 & -1
\end{array}\right] \\
& m=1 \\
& T_{1,1}=\sqrt{\frac{3}{10}}\left[\begin{array}{rrrr}
0 & -1 & 0 & 0 \\
0 & 0 & -\frac{2}{\sqrt{3}} & 0 \\
0 & 0 & 0 & -1 \\
0 & 0 & 0 & 0
\end{array}\right] \quad T_{2,1}=\sqrt{\frac{1}{2}}\left[\begin{array}{rrrr}
0 & -1 & 0 & 0 \\
0 & 0 & 0 & 0 \\
0 & 0 & 0 & 1 \\
0 & 0 & 0 & 0
\end{array}\right] \quad T_{3,1}=\frac{1}{\sqrt{5}}\left[\begin{array}{rrrr}
0 & -1 & 0 & 0 \\
0 & 0 & \sqrt{3} & 0 \\
0 & 0 & 0 & -1 \\
0 & 0 & 0 & 0
\end{array}\right] \\
& m=2 \\
& T_{2,2}=\sqrt{\frac{1}{2}}\left[\begin{array}{llll}
0 & 0 & 1 & 0 \\
0 & 0 & 0 & 1 \\
0 & 0 & 0 & 0 \\
0 & 0 & 0 & 0
\end{array}\right] \quad T_{3,2}=\sqrt{\frac{1}{2}}\left[\begin{array}{rrrr}
0 & 0 & 1 & 0 \\
0 & 0 & 0 & -1 \\
0 & 0 & 0 & 0 \\
0 & 0 & 0 & 0
\end{array}\right] \\
& m=3 \\
& T_{3,3}=\left[\begin{array}{rrrr}
0 & 0 & 0 & -1 \\
0 & 0 & 0 & 0 \\
0 & 0 & 0 & 0 \\
0 & 0 & 0 & 0
\end{array}\right]
\end{aligned}
$$

Figura 56: Tensores irredutíveis $\mathrm{T}_{l m}$ de rank $l$ e ordem $m$ para o spin 3/2. 

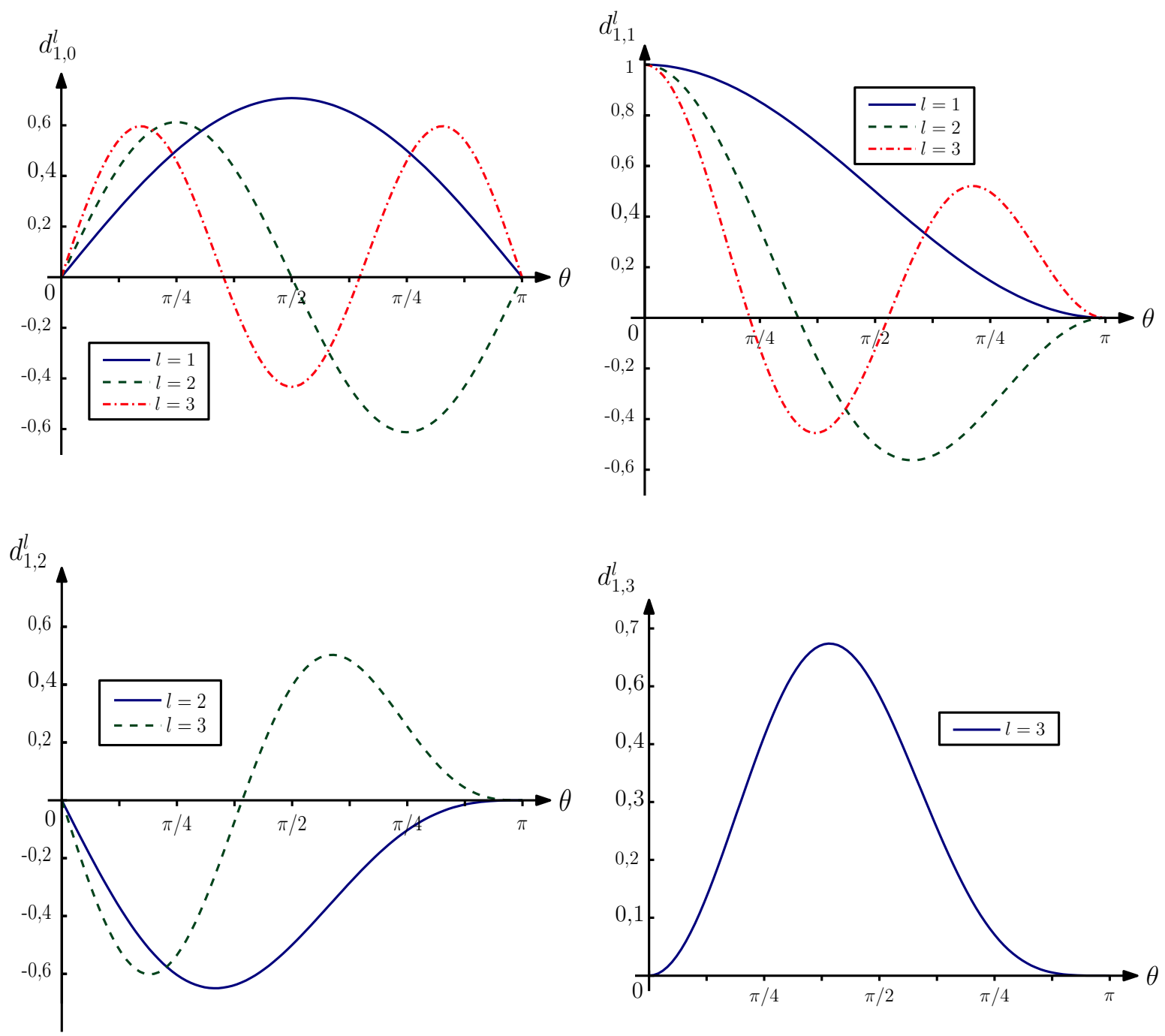

Figura 57: Funções $d_{1, m}^{l}$ de Wigner para o spin 3/2. 